WSRC-TR-2001-00277

\title{
Tank 37H Salt Removal Batch Process and Salt Dissolution Mixing Study
}

by

K. C. Kwon

Westinghouse Savannah River Company

Savannah River Site

Aiken, South Carolina 29808

M. R. Poirier

This paper was prepared in connection with work done under the above contract number with the U. S. Department of Energy. By acceptance of this paper, the publisher and/or recipient acknowledges the U. S. Government's right to retain a nonexclusive, royalty-free license in and to any copyright covering this paper, along with the right to reproduce and to authorize others to reproduce all or part of the copyrighted paper. 
This document was prepared in conjunction with work accomplished under Contract No.

DE-AC09-96SR18500 with the U.S. Department of Energy.

\section{DISCLAIMER}

This report was prepared as an account of work sponsored by an agency of the United States Government. Neither the United States Government nor any agency thereof, nor any of their employees, makes any warranty, express or implied, or assumes any legal liability or responsibility for the accuracy, completeness, or usefulness of any information, apparatus, product or process disclosed, or represents that its use would not infringe privately owned rights. Reference herein to any specific commercial product, process or service by trade name, trademark, manufacturer, or otherwise does not necessarily constitute or imply its endorsement, recommendation, or favoring by the United States Government or any agency

thereof. The views and opinions of authors expressed herein do not necessarily state or reflect those of the United States Government or any agency thereof.

This report has been reproduced directly from the best available copy.

Available for sale to the public, in paper, from: U.S. Department of Commerce, National Technical Information Service, 5285 Port Royal Road, Springfield, VA 22161, phone: (800)

553-6847, fax: (703) 605-6900, email: orders@ntis.fedworld.gov online ordering: http://www.ntis.gov/ordering.htm

Available electronically at http://www.doe.gov/bridge

Available for a processing fee to U.S. Department of Energy and its contractors, in paper, from: U.S. Department of Energy, Office of Scientific and Technical Information, P.O. Box 62, Oak Ridge, TN 37831-0062, phone: (865 ) 576-8401, fax: (865) 576-5728, email: reports@ adonis.osti.gov 
Keywords: Tank 37H, Salt

Dissolution, Heat Transfer

\title{
Tank 37H Salt Removal Batch Process and Salt Dissolution Mixing Study
}

\author{
Ki C. Kwon \\ Michael R. Poirier
}

July 17, 2001
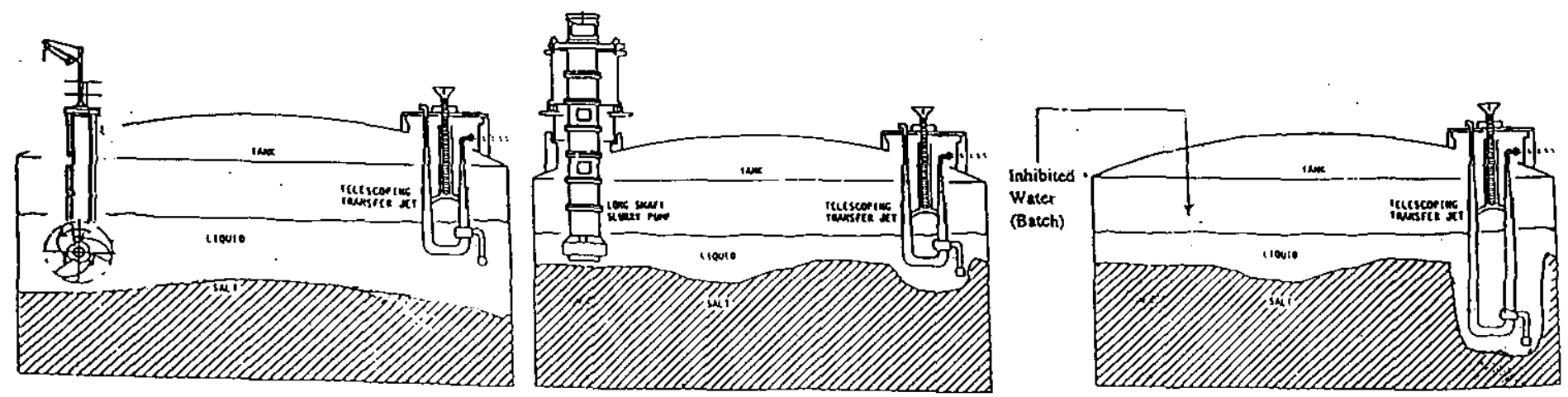
WSRC-TR-2001-00277, Rev. 0

APPROVAL

Authors:

Design Check

Customer

Management
Kichtoun 7/18/01

K. C. Kwon, WHMP

Truman PIPeisia
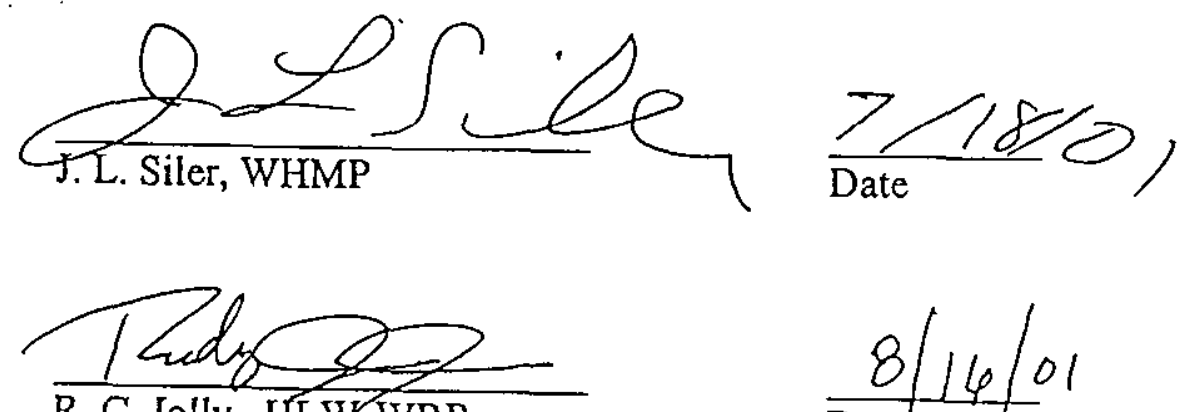

R. C. Jolly, HLW-WRP

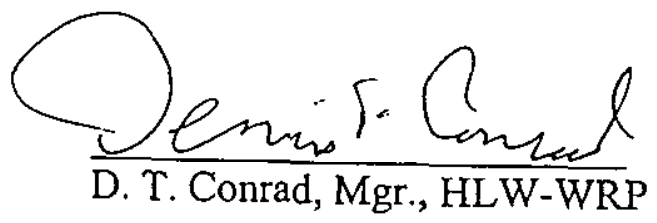

$\frac{\text { i). B. Van Re et }}{\text { W. B. Van Pelt, Mgr., WHMP }}$

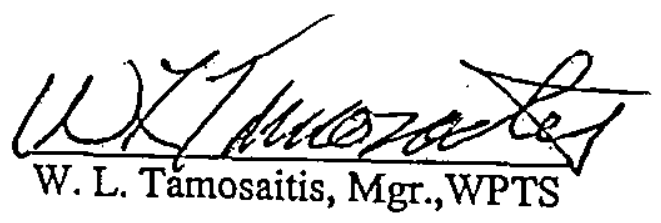

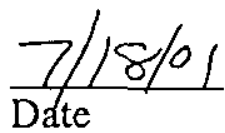

$\frac{8 / 16 / 01}{\text { Date }}$

$\frac{8 / 16 / 01}{\text { Date }}$

$7-18-01$

Date

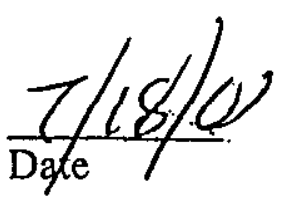

1 
Table of Contents

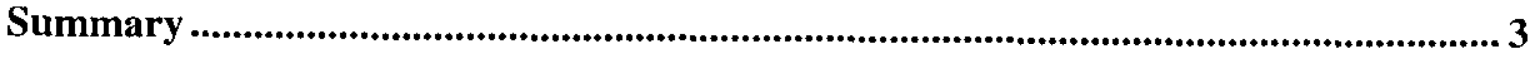

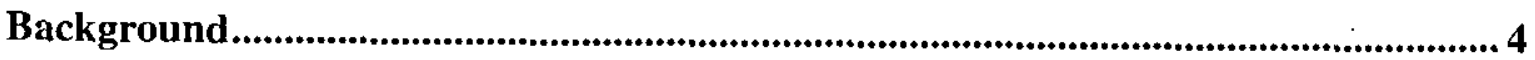

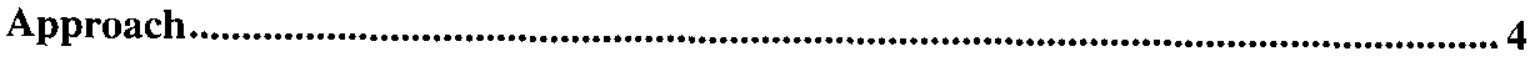

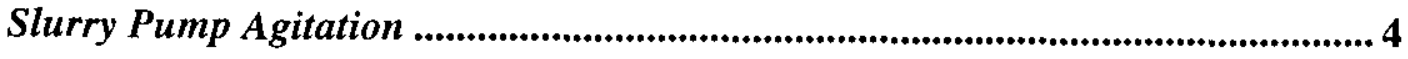

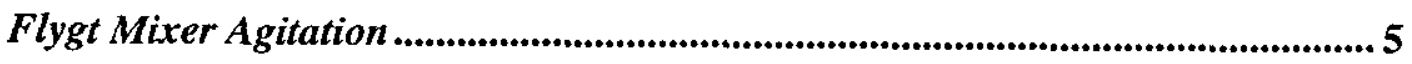

Multiple Mixers ..............................................................................................6

Modified Density Gradient .........................................................................6 6

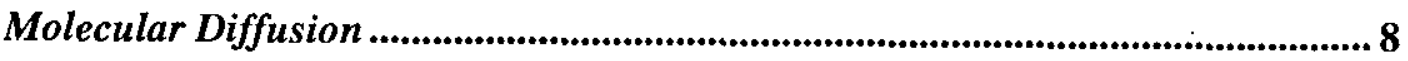

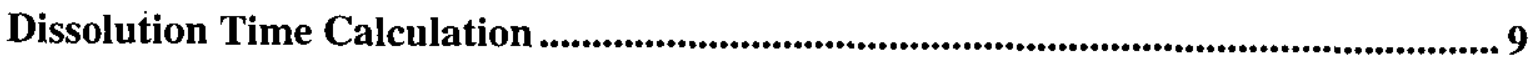

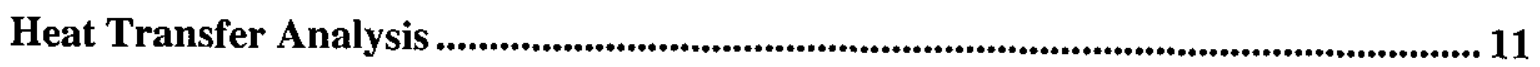

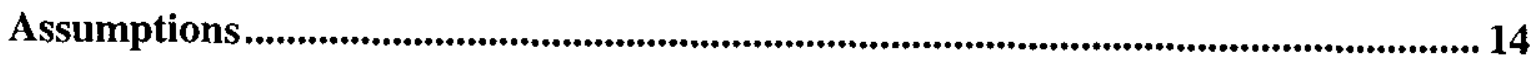

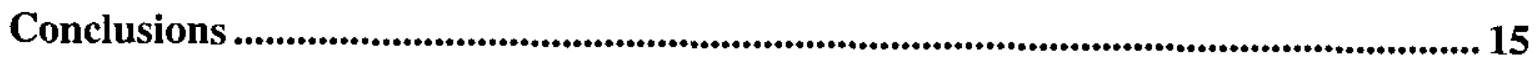

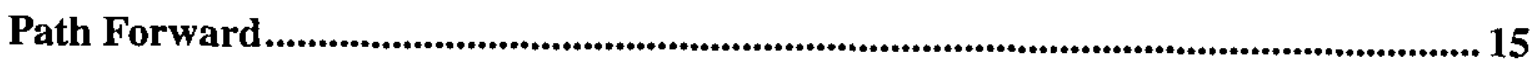

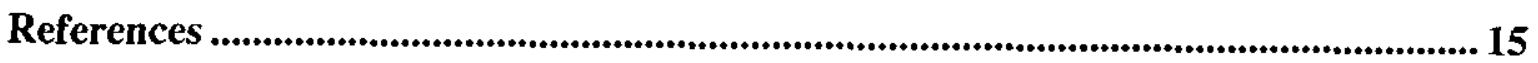

Appendix 1. Selected Sketches, Test Data and Example Calculations........................ 17

Appendix 2. Dissolution Calculations.......................................................................... 33

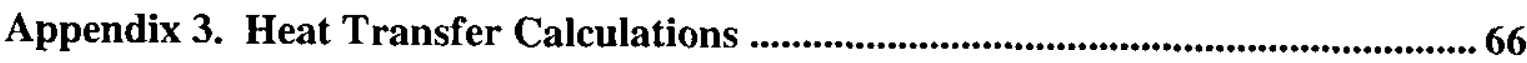




\section{Summary}

SRS High Level Waste (HLW) wishes to use Tank $37 \mathrm{H}$ as the receipt tank for the $3 \mathrm{H}$ Evaporator concentrate. Prior to using Tank $37 \mathrm{H}$ as the $3 \mathrm{H}$ Evaporator concentrate receipt tank, HLW must remove 50 inches of salt cake from the tank. They requested SRTC to evaluate various salt removal methods for Tank $37 \mathrm{H}$. These methods include slurry pumps, Flygt mixers, the modified density gradient (MDG) method, and molecular diffusion. The authors reviewed previous test and operating data as well as the technical literature to estimate the salt removal rate by each method. They considered 16 different combinations of agitation and inhibited water temperature, and calculated the dissolution time by each method. The results are shown below. They are based on the assumption of constant inhibited water temperature inside the tank during dissolution. In reality, the temperature of the supernate and salt cake will change during the process. The table shows the range of dissolution rates calculated, the dissolution rate used in the calculations, dissolution time, fraction of process time for dissolution, and total process time.

Dissolution Method

1. No Agitation $\left(25^{\circ} \mathrm{C}\right)$

2. $\mathrm{MDG}\left(25^{\circ} \mathrm{C}\right)$

3. $\mathrm{MDG}\left(50^{\circ} \mathrm{C}\right)$

4. $\mathrm{MDG}\left(75^{\circ} \mathrm{C}\right)$

5. One Flygt Mixer $\left(25^{\circ} \mathrm{C}\right)$

6. Two Flygt Mixers $\left(25^{\circ} \mathrm{C}\right)$

7. One Flygt Mixer $\left(50^{\circ} \mathrm{C}\right)$

8. One Slurry Pump $\left(25^{\circ} \mathrm{C}\right)$

9. Three Flygt Mixers $\left(25^{\circ} \mathrm{C}\right)$

10. One Flygt mixer $\left(75^{\circ} \mathrm{C}\right)$

11. Two Flygt Mixers $\left(50^{\circ} \mathrm{C}\right)$

12. One Slurry Pump $\left(50^{\circ} \mathrm{C}\right)$

13. Three Flygt Mixers $\left(50^{\circ} \mathrm{C}\right)$

14. Two Flygt Mixers $\left(75^{\circ} \mathrm{C}\right)$

15. One Slurry Pump $\left(75^{\circ} \mathrm{C}\right)$

16. Three Flygt Mixers $\left(75^{\circ} \mathrm{C}\right)$

$\begin{array}{ccc}\text { Dissolution } & \text { Dis. Rate } & \begin{array}{c}\text { Dissolution } \\ \text { Rate (gpm) }\end{array} \\ \begin{array}{cc}\text { used (gpm) } \\ \text { Time (days) }\end{array} \\ 1.18-2.47 & 1.83 & 117 \\ 1.25-5.84 & 3.55 & 74 \\ 1.78-8.32 & 5.05 & 60 \\ 2.33-10.80 & 6.56 & 53 \\ 2.0-4.0 & 3.0 & 82 \\ 2.8-5.7 & 4.2 & 67 \\ 2.8-5.8 & 4.3 & 66 \\ 3.0-7.0 & 5.0 & 61 \\ 3.5-6.9 & 5.2 & 59 \\ 3.7-7.4 & 5.6 & 57 \\ 4.0-8.0 & 6.1 & 55 \\ 4.3-9.9 & 7.1 & 51 \\ 4.9-9.8 & 7.4 & 50 \\ 5.3-10.5 & 8.6 & 47 \\ 5.6-13.0 & 9.3 & 46 \\ 6.4-12.9 & 10.6 & 43\end{array}$

$\begin{array}{cc}\begin{array}{c}\text { Dissolution Time } \\ \text { (\% of Total) }\end{array} & \begin{array}{c}\text { Total Process } \\ \text { Time (months) }\end{array} \\ 54 & 7.0 \\ 46 & 5.3 \\ 41 & 4.9 \\ 37 & 4.6 \\ 46 & 5.9 \\ 41 & 5.4 \\ 40 & 5.4 \\ 38 & 5.2 \\ 38 & 5.1 \\ 37 & 5.1 \\ 36 & 5.0 \\ 34 & 4.9 \\ 34 & 4.8 \\ 33 & 4.7 \\ 32 & 4.7 \\ 31 & 4.6\end{array}$

The conclusions from this work are the following:

- A single Flygt mixer with tank supernate temperature of $25^{\circ} \mathrm{C}$ should be sufficient to dissolve and remove 50 inches of salt cake within six months. Increasing the supernate temperature or adding additional Flygt mixers will decrease the time to dissolve the salt cake.

- A single slurry pump with tank supernate temperature of $25^{\circ} \mathrm{C}$ also should be sufficient to dissolve and remove 50 inches of salt cake within six months. Increasing supernate temperature or adding additional slurry pumps will decrease the time to dissolve the salt cake.

- Calculations predict that the modified density gradient method should be able to remove 50 inches of salt cake within six months, but uncertainty exists in predicting dissolution rates by this method (predicted dissolution rates varied between $1.25 \mathrm{gpm}$ and $5.84 \mathrm{gpm}$ at $25^{\circ} \mathrm{C}$ ). HLW should exercise caution when making decisions using calculated dissolution rates for this method.

- Molecular diffusion is unlikely to remove 50 inches of salt cake in six months. 
- If the inhibited water temperature is $25^{\circ} \mathrm{C}$, the actual tank supernate temperature will be higher and the salt dissolution rate faster than predicted by this analysis.

- If the inhibited water temperature is 50 or $75^{\circ} \mathrm{C}$, tank cooling will reduce the tank supernate temperature to $30-40^{\circ} \mathrm{C}$, which increases the dissolution time and minimizes the benefit of adding heated water to the tank. Including this effect, the dissolution rate with one Flygt mixer is $3.15 \mathrm{gpm}$ (rather than 4.3) for case 7 and $3.35 \mathrm{gpm}$ (rather than 5.6) for case 10 .

\section{Background}

Tank $30 \mathrm{H}$ is the receipt tank for concentrate from the $3 \mathrm{H}$ Evaporator. Tank $30 \mathrm{H}$ has had problems, such as cooling coil failure, which limit its ability to receive concentrate from the $3 \mathrm{H}$ Evaporator. SRS High Level Waste (HLW) wishes to use Tank $37 \mathrm{H}$ as the receipt tank for the $3 \mathrm{H}$ Evaporator concentrate. Prior to using Tank $37 \mathrm{H}$ as the $3 \mathrm{H}$ Evaporator concentrate receipt tank, HLW must remove 50 inches of salt cake from the tank. They requested SRTC to evaluate various salt removal methods for Tank $37 \mathrm{H}$. These methods include slurry pumps, Flygt mixers, the modified density gradient method, and molecular diffusion.

\section{Approach}

Four approaches were evaluated for removing salt cake from Tank $37 \mathrm{H}$ :

- Slurry pump agitation

- Flygt mixer agitation

- Modified density gradient

- Molecular diffusion

The authors reviewed previous test and operating data as well as the technical literature to estimate the salt removal rate by each method.

\section{Slurry Pump Agitation}

SRS performed a salt removal demonstration in Tank $19 \mathrm{~F}$ in 1980 and 1981 using long shaft vertical centrifugal pumps (i.e., slurry pumps). ${ }^{1}$ During the first dissolution batch, 172,000 gallons of salt cake was dissolved with 236,000 gallons of inhibited water in 350 hours using a single slurry pump ( $8.2 \mathrm{gpm}$ salt cake dissolution rate). During the dissolution, the supernate temperature was approximately $33^{\circ} \mathrm{C}$. In another salt removal application, SRTC examined the effect of temperature on salt cake dissolution rate and estimated it to be approximately $1.7 \% /{ }^{\circ} \mathrm{C}$ (using 200,000 gallons of dissolution water). ${ }^{2}$ Correcting for temperature, the estimated dissolution rate at $25^{\circ} \mathrm{C}$ is $7.1 \mathrm{gpm}$. During the second dissolution batch, 281,000 gallons of salt cake was dissolved with 442,000 gallons of inhibited water in 930 hours using a single slurry pump (5.0 gpm salt cake dissolution rate). The supernate temperature during batch 2 was $45^{\circ} \mathrm{C}$. Correcting for temperature, the estimated dissolution rate at $25^{\circ} \mathrm{C}$ is $3.3 \mathrm{gpm}$. Using this data, the estimated salt cake dissolution rate with a single slurry pump at $25^{\circ} \mathrm{C}$ is $3-7 \mathrm{gpm}$.

Tank 19F has no center column or cooling coils. Since Tank $37 \mathrm{H}$ has a center column and cooling coils, the actual dissolution rate will be less than in Tank 19F under the same conditions. Additionally, the volume of dissolution water in Tank 19F was greater than the volume of 
dissolution water to be used in Tank $37 \mathrm{H}$. Previous work showed increasing the dissolution water volume decreased the dissolution rate $^{2}$, so the smaller volume in Tank $37 \mathrm{H}$ would increase the dissolution rate over that observed in Tank 19F under the same conditions. These differences would affect the dissolution rate, but they are in opposite directions and should not change it dramatically.

The supernate density at the start of the Tank 19F salt dissolution demonstration was greater than 1.0. The reason is salt cake dissolution occurred prior to starting the slurry pumps. This dissolution was subtracted to calculate the overall dissolution rate in Tank 19F. Some dissolution will occur prior to starting mixing in Tank $37 \mathrm{H}$, but the amount cannot be quantified.

A salt cake dissolution rate of $3-7 \mathrm{gpm}$ at $25^{\circ} \mathrm{C}$ is probably a good estimate for a single standard slurry pump. The authors chose a dissolution rate of $5 \mathrm{gpm}$ at $25^{\circ} \mathrm{C}$ for this analysis.

\section{Flygt Mixer Agitation}

No salt removal testing or demonstrations have been performed at SRS to date with Flygt mixers. Salt dissolution rates with Flygt mixer agitation can be predicted from salt dissolution data in slurry pump agitated tanks. Previous SRTC work on salt dissolution assumed the process to be mass transfer limited and the mass transfer coefficient to be described by equation [1]

$$
\mathrm{K} \propto \sqrt{\frac{\tau_{\mathrm{w}}}{\rho}} \mathrm{Sc}^{-0.7} \propto \mathrm{U}_{\mathrm{j}} \mathrm{D}_{\mathrm{j}} \mathrm{Sc}^{-0.7}
$$

where $K$ is the mass transfer coefficient, $\tau_{w}$ is the shear stress at the salt cake surface, $S c$ is the Schmidt number, $U_{j}$ is the jet discharge velocity, and $D_{j}$ is the jet nozzle diameter. ${ }^{2,3}$ Since the Flygt mixer will behave as a turbulent jet, salt dissolution rates with Flygt mixers and slurry pumps can be compared by calculating the $U_{j} D_{j}$ for each device.

A standard slurry pump has a flow rate of $600 \mathrm{gpm} /$ nozzle, a nozzle diameter of 1.5 inches, and a nozzle velocity of $109 \mathrm{ft} / \mathrm{s}$. The $U_{j} D_{j}$ is $13.6 \mathrm{ft}^{2} / \mathrm{s}$. Since the slurry pump has two opposing nozzles, the total $U_{j} D_{j}$ is $27.2 \mathrm{ft}^{2} / \mathrm{s}$.

A model \# 4650 Flygt mixer has a flow rate of $10,700 \mathrm{gpm}$, a nozzle diameter of 22 inches, and a nozzle discharge velocity of $9 \mathrm{ft} / \mathrm{s}$. The $\mathrm{U}_{\mathrm{j}} \mathrm{D}_{\mathrm{j}}$ is $16.5 \mathrm{ft}^{2} / \mathrm{s}$. The Flygt mixer has a single discharge. The salt dissolution rate using Flygt mixers can be estimated from equation [2]

$$
R_{\text {Flygt }}=R_{\text {pump }}(16.5 / 27.2)=0.6 R_{\text {pump }}
$$

Using this approach, the estimated dissolution rate with a single Flygt mixer at $25^{\circ} \mathrm{C}$ is $2-4$ gpm. The effect of the center column and smaller dissolution water volume would be similar to their effects with the slurry pump. Modeling the Flygt mixer as a turbulent jet is a good assumption. A salt cake dissolution rate of $2-4 \mathrm{gpm}$ at $25^{\circ} \mathrm{C}$ is probably a good estimate for a single Flygt mixer. The authors chose a dissolution rate of $3 \mathrm{gpm}$ at $25^{\circ} \mathrm{C}$ for this analysis. 


\section{Multiple Mixers}

Using multiple Flygt mixers or slurry pumps will increase the dissolution rate. Two mixers will dissolve the salt cake faster than one mixer, but not necessarily twice as fast. Koh et. al. investigated the impact of the number of jets on jet mixing time. ${ }^{4}$ They found the mixing time to be inversely proportional to the square root of the number of jets. Using that work, one assumes the salt cake dissolution rate is proportional to the square root of the number of mixers or pumps.

\section{Modified Density Gradient}

Another method for dissolving salt cake is the modified density gradient method. In this method, inhibited water is added to one side of a waste tank at the same time, or sometimes before, the concentrated supernate is removed from the other side of the tank with a transfer jet that is mined into the salt cake. The current plan in Tank 37 is to have a mined well depth of 155 inches from the tank bottom.

In 1979 , SRS used a density gradient method to remove the salt from Tank 10 with a transfer jet that was mined into the salt to a depth of 36 inches from the tank bottom. , $^{8,10,11,12}$

SRTC investigated density driven salt dissolution techniques in 1996 . They found the method could be applied to SRS waste tanks, but the key step to performing this technique was to "maintain the solution level just above the top of the saltcake". 6 This report includes a laboratory test result of a modified density gradient (MDG) method using 3" wide x 36" long $\mathrm{x}$ $15^{\prime}$ high glass trough. The salt solution removal rate of MDG trough was $10 \mathrm{ml} / \mathrm{min}^{6}{ }^{6}$ If one assumes the dissolution rate does not change during scale up, the corresponding salt dissolution rate of Type IIIa Tank $37 \mathrm{H}$ is described by equation [3]

$$
\begin{aligned}
\text { Dissolution Rate } & =(10 \mathrm{ml} / \mathrm{min})(\text { Tank } 37 \mathrm{H} \text { surface area }) /(\text { Trough surface area }) /(2.4+1) \\
& =(10 \mathrm{~mL} / \mathrm{min} / 3786 \mathrm{~mL} / \mathrm{gal})\left(523.85 \mathrm{~m}^{2}\right) /\left(0.0697 \mathrm{~m}^{2}\right) / 3.4 \\
& =5.84 \mathrm{gpm}
\end{aligned}
$$

SRTC investigated density gradient methods at the bench-scale in 1979 . They found dissolution rate to be $1 / 4^{\text {th }}$ the rate measured with mechanical agitation. ${ }^{7,8}$ Assuming salt dissolution rate with the density gradient method at full-scale is $1 / 4^{\mathrm{th}}$ the slurry pump rate (or $5 \mathrm{gpm}$ average) dividing by 4 , one calculates a dissolution rate of $1.25 \mathrm{gpm}$ for Tank $37 \mathrm{H}$.

The references also show a laboratory test result of a density driven method using a $31 \mathrm{~cm}$ jar. ${ }^{7,8}$ The salt cake dissolution rate of the jar is $11 \mathrm{ml} / \mathrm{min}$. Assuming no change in the dissolution rate during scale up, the calculated salt dissolution rate of Tank $37 \mathrm{H}$ is calculated to be $6.13 \mathrm{gpm}$ in equation [4].

$$
\begin{aligned}
\text { Dissolution Rate } & =(11 \mathrm{ml} / \mathrm{min})(\text { Tank } 37 \mathrm{H} \text { surface area }) /(\text { Jar surface area }) /(2.4+1) \\
& =(11 \mathrm{~mL} / \mathrm{min} / 3786 \mathrm{~mL} / \mathrm{gal})\left(523.85 \mathrm{~m}^{2}\right) /\left(0.0731 \mathrm{~m}^{2}\right) / 3.4 \\
& =6.13 \mathrm{gpm}
\end{aligned}
$$


SRS performed a demonstration of the modified density gradient dissolution method in Tank 10 in $1979 .{ }^{8,9,10,11,12}$ Tank 10 is a Type I tank. It is $75 \mathrm{ft}$ in diameter, $24.5 \mathrm{ft}$ high, and has a capacity of 750,000 gallons. The tank contained a large salt mound (17 feet high) in one half of the tank. The other half also contained salt, but not at a uniform level. A level of 17 feet corresponds to 500,000 gallons. Since one half of the tank did not contain 17 feet of salt, the actual amount of salt cake in the tank was probably less than 500,000 gallons.

Supernate was observed between salt mounds in the tank. The supernate was probably saturated when the demonstration started. On two occasions, the tank received water from RBOF-RRF. The amount of water is not known. This water probably dissolved some of the salt cake in the tank and was near saturation when the demonstration started. Water was added to the tank to allow the transfer jet to be mined. The time this water was in the tank prior to the dissolution process beginning is not documented. The water would have dissolved some salt. Dissolution water was added batch wise to fill the tank to 206 inches ( $\sim 17$ feet) rather than continuously. ${ }^{\prime \prime}$ Some dissolution likely occurred as the water was added to the tank and while the water sat in the tank. The amount of dissolution is not known.

The teletype report of Tank 10 dated 4/30/79 stated that over 3 days of operation, the salt dissolution rate "appears to be about $0.01 \mathrm{ft} / \mathrm{hr}$ as was predicted by TNX test". This is equivalent to the salt dissolution rate of $5.42 \mathrm{gpm}$ with a supernate density of $1.3 \mathrm{~g} / \mathrm{cc}^{8}$ Equation [5] shows the calculation converting the change in salt cake elevation to salt cake dissolution rate.

However, the statement "appears to be about $0.01 \mathrm{ft} / \mathrm{hr}$ " raises uncertainty about the rigor with which the dissolution rate was calculated.

Dissolution Rate $=(0.01 \mathrm{ft} / \mathrm{hr})(12 \mathrm{in} / \mathrm{ft})(2710 \mathrm{gal} / \mathrm{in}) /(60 \mathrm{~min} / \mathrm{hr})=5.42 \mathrm{gpm}$

Another reference describes the dissolution rate to be $2.8 \mathrm{gpm} .{ }^{13}$ Because the tank conditions prior to the demonstration are not described and other effects such as water being in the tank prior to the demonstration starting could have contributed to dissolution, there is a great deal of uncertainty in the salt dissolution rate by the modified density gradient method. The reference does not provide the tank temperature during the demonstration. High Level waste should exercise caution when using this estimate. Additionally, the test conclusion indicates that solution chemistry (i.e., corrosion inhibitors) was difficult to control without tank mixing. ${ }^{12}$

Tank 10 Salt Removal Demonstration Details \& Teletype Data also provides additional dissolution test data from 4/25 through 5/2979. ${ }^{11}$ The dissolution process was performed for 35 days. According to the data recorded, 140,046 gallons of salt cake was dissolved. Dividing the amount of salt dissolved by the demonstration time ( 35 days) yields a salt cake dissolution rate of $2.8 \mathrm{gpm}$.

Based on the data obtained for salt dissolution by density gradient methods, the authors decided to perform the analysis with a dissolution rate of $3.55 \mathrm{gpm}$ (the mid-point of 1.25 and 5.84) at $25^{\circ} \mathrm{C}$. This approach makes a number of assumptions and extrapolations, so High Level Waste should exercise caution when using calculated dissolution rates for the modified density gradient method. 


\section{Molecular Diffusion}

Another method for salt dissolution is molecular diffusion. With this method, inhibited water is added to the tank, salt cake dissolves, and supernate mixing occurs by molecular diffusion.

Since thermal gradients will exist in waste tanks, thermal diffusion must also be considered. The thermal gradients will cause density variations that will increase the mass transfer rate. The thermal convection mass transfer coefficient can be calculated by using an analogy with thermal convection heat transfer that is described by equation [6]. ${ }^{14}$

$$
\frac{\mathrm{kL}}{\mathrm{D}}=0.13\left(\frac{\mathrm{gL}{ }^{3} \rho^{2} \Delta \rho}{\rho \mu^{2}}\right)^{1 / 3}\left(\frac{\mu}{\rho \mathrm{D}}\right)^{1 / 3}
$$

In equation [3], $\mathrm{k}$ is the mass transfer coefficient, $\mathrm{L}$ is the liquid depth, $\mathrm{D}$ is diffusivity, $\mathrm{g}$ is the acceleration due to gravity, $\rho$ is supernate density, and $\mu$ is the supernate viscosity. The temperature difference is assumed to be $1^{\circ} \mathrm{C}$. Once the mass transfer coefficient is calculated, the mass transfer rate can be calculated with equation [7].

$$
\mathrm{J}=\mathrm{k} A \Delta \mathrm{C}
$$

In equation [4], $\mathrm{A}$ is the surface area and $\Delta \mathrm{C}$ is the concentration gradient. Table 1 shows the input parameters for equations [6] and [7].

Using the input parameters from Table 1 and equations [6] - [7], the salt dissolution rate by thermal diffusion is calculated to be $2.47 \mathrm{gpm}$ for $2.4: 1$ dissolution ratio (2.4 gal. water to 1 gal salt cake dissolved).

\begin{tabular}{|c|c|}
\hline Parameter & Value \\
\hline $\mathrm{D}\left(\mathrm{cm}^{2} / \mathrm{s}\right)$ & $1.57 \times 10^{-5}$ \\
\hline $\mathrm{L}$ (in) & 50 \\
\hline$\rho(\mathrm{g} / \mathrm{cc})$ & 1.3919 \\
\hline$\mu$ (cp) & 2.42 \\
\hline$v\left(\mathrm{~cm}^{2} / \mathrm{s}\right)$ & 0.0174 \\
\hline$\Delta \mathrm{T}\left({ }^{\circ} \mathrm{C}\right)$ & 1 \\
\hline$\Delta \rho(\mathrm{g} / \mathrm{cc})$ & 0.00038 \\
\hline $\mathrm{g}\left(\mathrm{cm} / \mathrm{s}^{2}\right)$ & 980 \\
\hline$\Delta \mathrm{C}(\mathrm{g} / \mathrm{cc})$ & 0.2 \\
\hline $\mathrm{A}\left(\mathrm{cm}^{2}\right)$ & $5.27 \times 10^{6}$ \\
\hline
\end{tabular}

\section{Table 1. Input Parameters for Thermal Diffusion Calculation}

In 1981, SRTC conducted a horizontal plane dissolution test, which is similar to molecular diffusion, using a four liter beaker with 15 centimeter diameter. ${ }^{8}$ The measured salt dissolution rate was $0.51 \mathrm{~mL} / \mathrm{min}$, which corresponds to a dissolution rate of 1.18 gpm in Tank $37 \mathrm{H}$. The authors used $1.18 \mathrm{gpm}, 2.47 \mathrm{gpm}$, and $1.83 \mathrm{gpm}$ (the average) to calculate salt dissolution times by this method. 
This approach makes a number of assumptions and extrapolations. It has not been experimentally validated. Therefore, SRS HLW should exercise caution when using this data to predict salt removal rates by molecular diffusion.

\section{Dissolution Time Calculation}

SRTC used the analysis described above to calculate the time to dissolve 50 inches of salt cake by various methods. Table 2 shows the methods considered. The table shows the range of dissolution rates calculated for each method, as well as the dissolution rate used to calculate he total dissolution process time. The dissolution rate used for the calculations with the slurry pumps and Flygt mixers was determined by using the average dissolution rate in Tank 19 at $25^{\circ} \mathrm{C}$, modifying it if necessary to calculate the dissolution rate with a Flygt mixer, and correcting the rate for temperature and the number of mixers. The results of calculation for dissolution time with MDG and other methods are shown in Table 2.

To calculate the time to dissolve 50 inches of salt cake, one must also consider the time required to add inhibited water, the salt dissolution time, the time to transfer supernate from the tank, and sampling and preparation time. SRTC made the following assumptions to calculate the salt dissolution time. Tank $37 \mathrm{H}$ will contain 269.5 inches of crystallized salt cake and 81.69 inches of saturated supernate when the supernate is removed from the tank. This supernate will be saturated with salt. The ratio of dissolved salt cake to inhibited water in the supernate removed from the tank is 1:2.4. HLW can add a maximum of 40 inches of inhibited water to the tank for each dissolution batch. HLW can add 8000 gallons of inhibited water in a 12-hour shift (the addition rate is $11.1 \mathrm{gpm}$ ). Seven days was added to the dissolution time for each batch to be conservative. The jet transfer rate of non-MDG to TK 26 is $60 \mathrm{gpm}$. As shown in Table 2, the jet transfer rate of MDG to Tank 26 are 4.25 to $36.73 \mathrm{gpm}$. Sampling and preparation time is 240 hours per dissolution batch.

Table 3 shows a number of different techniques can be used to dissolve the salt cake in Tank $37 \mathrm{H}$. The spreadsheets used to perform the calculations are included in Appendix 1. The modified density gradient method with a transfer jet in the deeply mined well could dissolve the salt cake faster than a single Flygt mixer and horizontal plane or molecular diffusion dissolution. Increasing the supernate temperature of all methods or increasing the number of mixers decreases the dissolution time. The table also shows the fraction of dissolution time required for mixing is $30-54 \%$ of the total dissolution time. HLW can improve the salt dissolution rate by reducing the time for other steps (e.g., water addition, supernate removal, and sampling). Some dissolution will occur while inhibited water is added to the tank, which will reduce the overall salt dissolution time. The exact reduction in dissolution time cannot be quantified.

The authors have more confidence in the predicted dissolution times with Flygt mixers and slurry pumps than they do in the predicted dissolution times with molecular diffusion (MD) and the modified density gradient (MDG) method. There are a number of uncertain thermal effects on MD and unknown salt cake internal structural porosity or moisture permeability and undefined drain passage pattern which could change with time, temperature, transfer jet suction condition, water-salt cake level and other MDG method parameters. 
Table 2. Salt Cake Dissolution Methods

Method

Dissolution Rate Transfer Rate to Total Process

Range (gpm) TK 26 (gpm) Time (Months)

No Agitation 25C Low

No Agitation 25C Mid.

No Agitation $25 \mathrm{C} \mathrm{High}$

MDG 25C Low

MDG 25C Mid-value

MDG 25C High

MDG 50C Low

MDG 50C Mid-value

MDG 50C High

MDG 75C Low

MDG 75C Mid-Value

MDG 75C High

1 Flygt Mixer 25C Low

1 Flygt Mixer 25C Mid.

1 Flygt Mixer 25C High

1 Flygt Mixer 50C (Mid)

1 Flygt Mixer 75C

2 Flygt Mixers 25C

2 Flygt Mixers 50C

2 Flygt Mixers 75C

3 Flygt Mixers 25C

3 Flygt Mixers 50C

3 Flygt Mixers 75C

1 Slurry Pump 25C

1 Slurry Pump 50C

1 Slurry Pump 75C
1.18

1.83

2.47

1.25

3.55

5.84

1.78

5.05

8.32

2.33

6.56

10.8

2.0

3.0

4.0
60

8.63

60

60

7.03

6.27

4.25

8.08

12.05

5.32

19.86

4.73

6.05

6.81

17.17

4.87

28.29

4.45

7.91

6.11

23.32

36.73

4.63

4.31

60

60

6.78

5.89

60

5.45

$$
2.8-5.7(4.3)
$$

60

5.4

$$
3.7-7.4(5.6)
$$

60

5.1

$$
2.8-5.7(4.2)
$$

$4.0-8.0(6.1)$

$5.3-10.5(8.6)$

60

60

60

5.4

5.0

4.7

$$
3.5-6.9(5.2)
$$

60

5.1

$$
4.9-9.8(7.4)
$$

$6.4-12.9(10.6)$
60

60

$$
\begin{aligned}
& 3.0-7.0(5.0) \\
& 4.3-9.9(7.1)
\end{aligned}
$$

60

60

60

4.8

4.6

$5.6-13.0(9.3)$
5.2

4.9

4.7

Using these assumptions, SRTC calculated the dissolution time by each method in Table 2 . Table 3 shows the results. 


\begin{tabular}{lccccc}
\hline Method of Dissolution & $\begin{array}{c}\text { Water Temp. } \\
\left({ }^{\circ} \mathrm{C}\right)\end{array}$ & $\begin{array}{c}\text { Dissolution } \\
\text { Rate }(\mathrm{gpm})\end{array}$ & $\begin{array}{c}\text { Dissolution } \\
\text { Time (days) }\end{array}$ & $\begin{array}{c}\text { Dissolution Time } \\
\text { (\% of Total) }\end{array}$ & $\begin{array}{c}\text { Total Process } \\
\text { Time (months) }\end{array}$ \\
\hline 1. No Agitation & 25 & 1.83 & 116.8 & 54.4 & 7.0 \\
2. MDG & 25 & 3.55 & 73.8 & 46.0 & 5.3 \\
3. MDG & 50 & 5.05 & 60.2 & 40.5 & 4.9 \\
4. MDG & 75 & 6.56 & 52.8 & 37.4 & 4.6 \\
5. One Flygt Mixer & 25 & 3.0 & 82.2 & 45.7 & 5.9 \\
6. Two Flygt Mixers & 25 & 4.2 & 66.7 & 40.5 & 5.4 \\
7. One Flygt Mixer & 50 & 4.3 & 65.8 & 40.0 & 5.4 \\
8. One Slurry Pump & 25 & 5.0 & 60.5 & 38.3 & 5.2 \\
9. Three Flygt Mixers & 25 & 5.2 & 59.3 & 37.8 & 5.1 \\
10. One Flygt mixer & 75 & 5.6 & 57.0 & 36.9 & 5.1 \\
11. Two Flygt Mixers & 50 & 6.1 & 54.6 & 35.9 & 5.0 \\
12. One Slurry Pump & 50 & 7.1 & 50.9 & 34.3 & 4.9 \\
13. Three Flygt Mixers & 50 & 7.4 & 50.0 & 33.9 & 4.8 \\
14. Two Flygt Mixers & 75 & 8.6 & 46.9 & 32.5 & 4.7 \\
15. One Slurry Pump & 75 & 9.3 & 45.5 & 31.8 & 4.7 \\
16. Three Flygt Mixers & 75 & 10.6 & 43.3 & 30.8 & 4.6 \\
\hline
\end{tabular}

\section{Heat Transfer Analysis}

One of the authors has developed a heat transfer model which describes heating and cooling in SRS High Level Waste Tanks. ${ }^{15,16,17.18}$ The model is described by equation [5]

$$
c_{p} \rho V(d T / d t)=Q_{d e c}+Q_{j p m}+Q_{p u r}+Q_{c o i}+Q_{a n a}+Q_{u n s}+Q_{v w l}+Q_{s e n}+Q_{t s r}
$$

where $c_{p}$ is the specific heat of the liquid or solid phase, $\rho$ is the density of the liquid or solid phase, $V$ is the liquid or solid phase volume, $T$ is bulk temperature, $t$ is time, $Q_{\text {dec }}$ is radionuclide decay heat, $Q_{j p m}$ is heat input from a steam jet or slurry pump, $Q_{p u r}$ is the heat removed by evaporation and purge gas, $\mathrm{Q}_{\mathrm{coi}}$ is the heat removed by submerged and vapor space cooling coil water, $Q_{\text {ana }}$ is the heat removed by annulus air passing through the tank bottom cooling slots, $Q_{u n s}$ is the heat removed by underground soil, $\mathrm{Q}_{\mathrm{vwl}}$ is the heat flow through the vertical tank wall, $\mathrm{Q}_{\mathrm{sen}}$ is the sensible liquid heat flow into or out of the tank, and $\mathrm{Q}_{\mathrm{tsr}}$ is the heat absorbed by $\operatorname{tank}$ structures.

This model evaluates equation [5] in discrete time steps. It evaluates heat inputs and outputs on the right side of equation [5], and calculates the new heat balance and temperature at the end of the time step. This process is repeated for a time determined by the user. SRS HLWE has compared the model predictions with actual waste tank temperatures to validate the model. The model is applicable to all types of SRS Tank Farm waste tanks (Types I, II, III, IIIa, and IV). Figure 1 shows a schematic of how the model is applied to SRS Waste Tanks. It has been approved by SRS HLWM for calculating waste tank tempertures. ${ }^{15}$

The authors employed the model to calculate the supernate and salt cake temperature during the entire dissolution process. The initial waste tank supernate temperature is assumed to be $37^{\circ} \mathrm{C}$. If the inhibited water added to the tank has a temperature of $25^{\circ} \mathrm{C}$, the supernate temperature will decrease as the inhibited water is added. If the inhibited water added to the tank has a 
temperature of $50^{\circ} \mathrm{C}$ or $75^{\circ} \mathrm{C}$, the supernate temperature will increase as the inhibited water is added. Since salt dissolution is an endothermic process, the supernate temperature decreases during salt dissolution. Once salt dissolution is complete, radionuclide decay heat will increase the supernate temperature. The supernate temperature will, in most cases, rise slightly during transfer of supernate from Tank $37 \mathrm{H}$. Table 4 shows the calculated tank supernate temperature following inhibited water addition, àt the end of salt dissolution, at the end of mixing, and the peak supernate temperature during batch 1 and batch 4 for each dissolution method. The table shows that when the inhibited water temperature is $50^{\circ} \mathrm{C}$ or $75^{\circ} \mathrm{C}$, the actual supernate temperature will be much lower. When the inhibited water temperature is $25^{\circ} \mathrm{C}$, the tank supernate temperature will be higher.

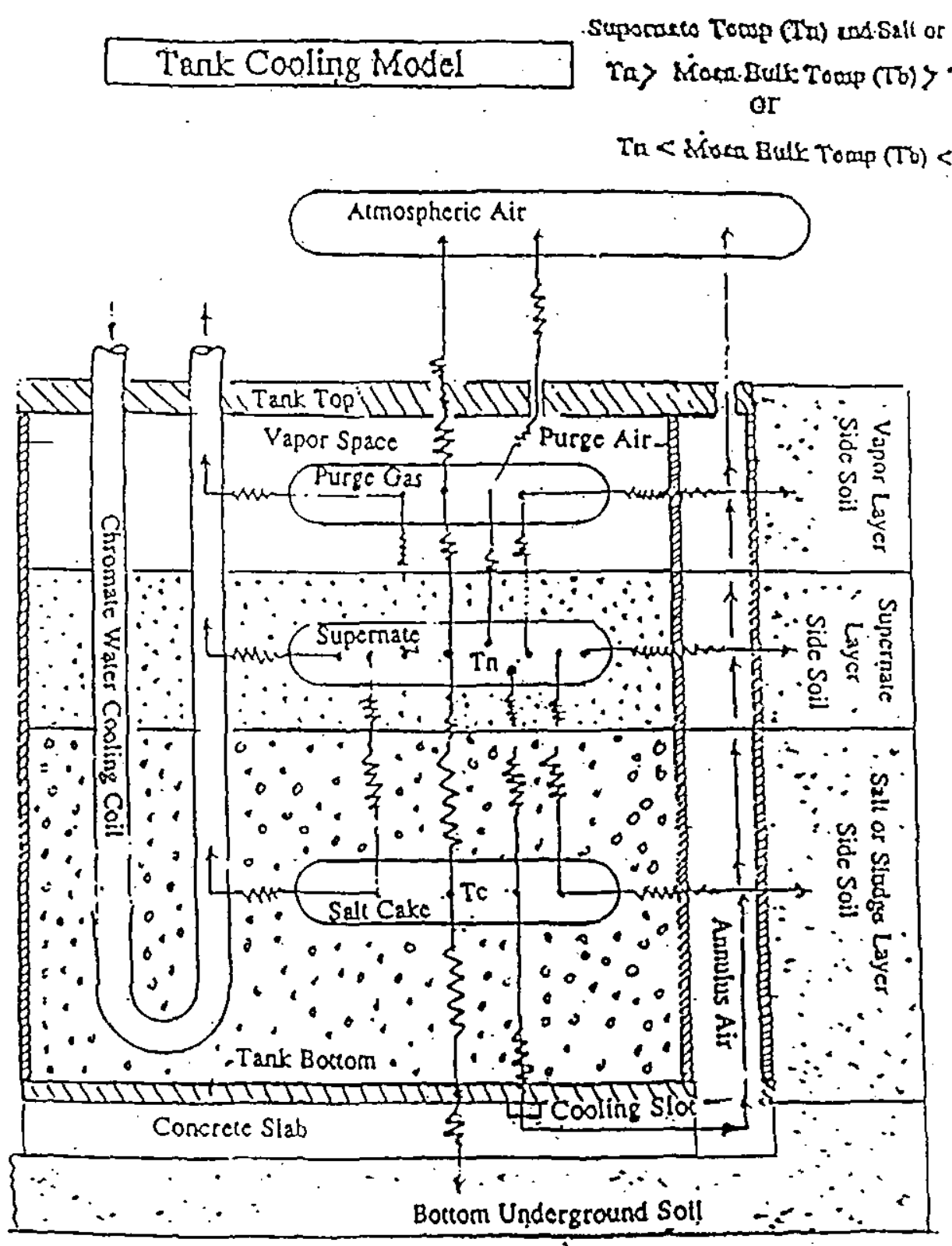

Figure 1. Schematic of Tank Cooling Model 
When dissolving salt cake with one Flygt mixer and $25^{\circ} \mathrm{C}$ inhibited water, the supernate temperature will vary between $37^{\circ} \mathrm{C}$ and $27^{\circ} \mathrm{C}$ during the process. The supernate temperature at the end of the salt dissolution for the four batches was $34^{\circ} \mathrm{C}, 31.5^{\circ} \mathrm{C}, 29.5^{\circ} \mathrm{C}$, and $28^{\circ} \mathrm{C}\left(31^{\circ} \mathrm{C}\right.$ average). Since this temperature is higher than $25^{\circ} \mathrm{C}$, the actual salt dissolution rate will be slightly higher than predicted by these calculations. Similar results are expected for other dissolution methods with $25^{\circ} \mathrm{C}$ inhibited water addition.

When dissolving salt cake with one Flygt mixer and $50^{\circ} \mathrm{C}$ inhibited water, the supernate temperature will vary between $38^{\circ} \mathrm{C}$ and $32^{\circ} \mathrm{C}$ during the process. The supernate temperature at the end of the salt dissolution for the four batches was $36^{\circ} \mathrm{C}, 34.5^{\circ} \mathrm{C}, 33.5^{\circ} \mathrm{C}$, and $33^{\circ} \mathrm{C}\left(34^{\circ} \mathrm{C}\right.$ average). Since this temperature is lower than $50^{\circ} \mathrm{C}$, the actual salt dissolution rate will be less than predicted by these calculations. At a supernate temperature of $34^{\circ} \mathrm{C}$, the calculated salt dissolution rate is $3.15 \mathrm{gpm}$. Similar results are expected for other dissolution methods with $50^{\circ} \mathrm{C}$ inhibited water addition.

Table 4. Tank 37H Supernate Temperature

\begin{tabular}{|c|c|c|c|c|c|}
\hline Method of Dissolution & $\begin{array}{c}\text { Water } \\
\text { Temp. }\left({ }^{\circ} \mathrm{C}\right) \\
\end{array}$ & $\begin{array}{c}\text { End IW Addition } \\
\text { Batch I } \\
\text { Batch } 4 \\
\end{array}$ & $\begin{array}{c}\text { Peak Temp. } \\
\text { Batch } 1 \\
\text { Batch } 4 \\
\end{array}$ & $\begin{array}{c}\text { End Dissolution } \\
\text { Batch } 1 \\
\text { Batch } 4 \\
\end{array}$ & $\begin{array}{c}\text { End Mixing } \\
\text { Batch 1 } \\
\text { Batch 4 }\end{array}$ \\
\hline \multirow[t]{2}{*}{ 1. No Agitation } & 25 & 36.0 & 37.0 & 33.6 & 34.3 \\
\hline & & 29.7 & 29.9 & 27.6 & 27.9 \\
\hline \multirow[t]{2}{*}{ 2. MDG } & 25 & 36.0 & 37.0 & 33.4 & 33.6 \\
\hline & & 29.1 & 29.1 & 26.5 & $27.0-$ \\
\hline \multirow[t]{2}{*}{ 3. MDG } & 50 & 38.0 & 38.0 & 35.0 & 35.0 \\
\hline & & 34.0 & 34.0 & 30.6 & 30.8 \\
\hline \multirow[t]{2}{*}{ 4. $\mathrm{MDG}$} & 75 & 40.1 & 40.1 & .37 .3 & 37.0 \\
\hline & & 39.9 & 40.0 & 36.6 & 36.2 \\
\hline \multirow[t]{2}{*}{ 5. One Flygt Mixer } & 25 & 36.0 & 37.0 & 35.6 & 35.9 \\
\hline & & 29.8 & 30.0 & 27.5 & 27.7 \\
\hline \multirow[t]{2}{*}{ 6. Two Flygt Mixers } & 25 & 36.5 & 37.0 & 33.7 & 34.0 \\
\hline & & 30.0 & 30.2 & 27.6 & 28.2 \\
\hline \multirow[t]{2}{*}{ 7. One Flygt Mixer } & 50 & 38.0 & 38.0 & 35.5 & 35.7 \\
\hline & & 35.4 & 35.4 & 32.5 & 32.7 \\
\hline \multirow[t]{2}{*}{ 8. One Slurry Pump } & 25 & 36.0 & 38.6 & 36.1 & 38.6 \\
\hline & & 37.9 & 40.6 & 37.8 & 40.6 \\
\hline \multirow[t]{2}{*}{ 9. Three Flygt Mixers } & 25 & 36.0 & 37.0 & 33.8 & 34.3 \\
\hline & & 30.4 & 30.7 & 28.1 & 28.8 \\
\hline \multirow[t]{2}{*}{ 10. One Flygt mixer } & 75 & 40.0 & 40.0 & 37.5 & 37.5 \\
\hline & & 40.8 & 40.8 & 37.7 & 37.7 \\
\hline \multirow[t]{2}{*}{ 11. Two Flygt Mixers } & 50 & 38.1 & 38.1 & 35.6 & 35.9 \\
\hline & & 35.6 & 35.6 & 32.8 & 33.2 \\
\hline \multirow[t]{2}{*}{ 12. One Slurry Pump } & 50 & 38.0 & 39.7 & 37.3 & 39.7 \\
\hline & & 42.2 & 43.7 & 41.1 & 43.7 \\
\hline \multirow[t]{2}{*}{ 13. Three Flygt Mixers } & 50 & 38.1 & 38.1 & 35.8 & 36.2 \\
\hline & & 36.2 & 36.2 & 33.5 & 34.0 \\
\hline \multirow[t]{2}{*}{ 14. Two Flygt Mixers } & 75 & 40.2 & 40.2 & 37.5 & 37.7 \\
\hline & & 41.3 & 41.3 & 38.1 & 38.3 \\
\hline \multirow[t]{2}{*}{ i5. One Slurry Pump } & 75 & 40.1 & 41.2 & 38.9 & 41.2 \\
\hline & & 47.3 & 47.3 & 45.6 & 47.9 \\
\hline \multirow[t]{2}{*}{ 16. Three Flygt Mixers } & 75 & 40.2 & 40.2 & 37.8 & 38.2 \\
\hline & & 42.2 & 42.2 & 39.3 & 39.6 \\
\hline
\end{tabular}


When dissolving salt cake with one Flygt mixer and $75^{\circ} \mathrm{C}$ inhibited water, the supernate temperature will vary between $41^{\circ} \mathrm{C}$ and $37^{\circ} \mathrm{C}$ during the process. The supernate temperature at the end of the salt dissolution for the four batches was $37.5^{\circ} \mathrm{C}, 37.7^{\circ} \mathrm{C}, 37.8^{\circ} \mathrm{C}$, and $37.8^{\circ} \mathrm{C}$ $\left(38^{\circ} \mathrm{C}\right.$ average). Since this temperature is lower than $75^{\circ} \mathrm{C}$, the actual salt dissolution rate will be less than predicted by these calculations. At a supernate temperature of $38^{\circ} \mathrm{C}$, the calculated salt dissolution rate is $3.35 \mathrm{gpm}$. Similar results are expected for other dissolution methods with $75^{\circ} \mathrm{C}$ inhibited water addition.

The heat transfer calculation results are shown in Appendix 2.

\section{Assumptions}

The authors made the following assumptions in performing the salt dissolution analysis:

- The effect of increasing tank supernate temperature on the dissolution rate is $1.7 \% /{ }^{\circ} \mathrm{C}$.

- The tank cooling coils have a small effect on the salt dissolution rate

- Using a smaller volume of dissolution water in Tank 37H than in Tank 19F has a small effect on the salt dissolution rate.

- The dissolution process is mass transfer limited.

- The discharge from a Flygt mixer behaves as a turbulent jet.

- The salt dissolution rate is proportional to the square root of the number of pumps or Flygt mixers.

- The ratio of dissolution fluid to salt cake is $2.4: 1$. This ratio is an average from the Tank 19 Salt Removal Demonstration.

- The ratio of the salt dissolution rate by the modified density gradient approach to the salt dissolution rate with mechanical agitation does not change during scale-up.

- The Colburn analogy can be used to describe thermal convection mass transfer.

- The thermal gradient in Tank $37 \mathrm{H}$ is $1^{\circ} \mathrm{C}$. This assumption is based on the temperature difference between the salt cake and supernate obtained from the HLW morning report.

- The saturated supernate density is $1.39 \mathrm{~g} / \mathrm{cc}$, which is the density of a saturated sodium nitrate solution.

- The saturated supernate viscosity is $2.42 \mathrm{cp}$., which is the density of a saturated sodium nitrate solution.

- Tank $37 \mathrm{H}$ contains 269.5 inches of salt cake.

- Tank $37 \mathrm{H}$ contains 81.69 inches of saturated supernate.

- HLW can add a maximum of 40 inches of inhibited water to the tank a one time.

- HLW can add 8000 gallons of inhibited water to the tank in one 12 hour shift.

- Seven days is added to the dissolution time in each batch for conservatism.

- The jet transfer rate is $60 \mathrm{gpm}$ for non-MDG dissolution cases.

- Sampling and preparation time is 240 hours for each dissolution batch.

- The initial waste tank supernate temperature is at $37^{\circ} \mathrm{C}$ 


\section{Conclusions}

The conclusions from this work are the following:

- A single Flygt mixer with tank supernate temperature of $25^{\circ} \mathrm{C}$ should be sufficient to dissolve and remove 50 inches of salt cake within six months. Increasing the supernate temperature or adding additional Flygt mixers will decrease the time to dissolve the salt cake.

- A single slurry pump with tank supernate temperature of $25^{\circ} \mathrm{C}$ also should be sufficient to dissolve and remove 50 inches of salt cake within six months. Increasing supernate temperature or adding additional slurry pumps will decrease the time to dissolve the salt cake.

- Calculations predict that the modified density gradient method should be able to remove 50 inches of salt cake within six months, but uncertainty exists in predicting dissolution rates by this method (predicted dissolution rates varied between $1.25 \mathrm{gpm}$ and $5.84 \mathrm{gpm}$ at $25^{\circ} \mathrm{C}$ ). HLW should exercise caution when making decisions using calculated dissolution rates for this method.

- Molecular diffusion is unlikely to remove 50 inches of salt cake in six months.

- If the inhibited water temperature is $25^{\circ} \mathrm{C}$, the actual tank supernate temperature will be higher and the salt dissolution rate faster than predicted by this analysis.

- If the inhibited water temperature is 50 or $75^{\circ} \mathrm{C}$, tank cooling will reduce the tank supernate temperature to $30-40^{\circ} \mathrm{C}$, which increases the dissolution time and minimizes the benefit of adding heated water to the tank. Including this effect, the dissolution rate with one Flygt mixer is $3.15 \mathrm{gpm}$ (rather than 4.3) for case 7 and $3.35 \mathrm{gpm}$ (rather than 5.6) for case 10 .

\section{Path Forward}

If HLW requires more rigorous estimates of the salt dissolution time, SRTC could reduce the uncertainty in the calculated dissolution time by performing the following work:

- Perform a more rigorous analysis of salt dissolution by the density gradient methods.

- Perform a more rigorous analysis of salt dissolution by molecular diffusion.

- Perform a more rigorous evaluation of changing supernate temperature in the tank when the inhibited water is heated prior to addition.

\section{References}

1. A. Q. Goslen, “Tank 19 Salt Removal”, DPSP-84-17-7, August 1986.

2. M. R. Poirier, "Recommended Slurry Pump Operating parameters in Tank 25", WSRC-RP95-00723, July 31, 1995.

3. M. R. Poirier, "Calculating Salt Dissolution Rates in Tank 41", X-CLC-H-00019, January 11, 1995.

4. S. T. Koh, S. K. Setsuro Y. Tada, T. Takahashi, T. Aragaki, and I. Yamada, "Jet Mixing of Liquids with a Rotating Nozzle around the Axis of a Cylindrical Vessel", J. Chem. Eng. Japan, 23, no. 4, 1990, pp. 463-467.

5. J. N. Brooke, "Report \#GL-97-September 1997 from the Waterways Experiment Station of the US Army Cops of Engineers Titled: Interstitial Fluid Displacement (IFD) for Preferential Recover of Cesium from Saltcake", SRT-WHM-98-0017, February 5, 1998. 
6. B. J. Wiersma, "An Investigation of Density Driven Salt Dissolution Techniques", WSRCTR-96-0160, August 1996.

7. D. L. Kiser, "Bench-Scale Investigation of Different Concepts for Waste Tank Salt Dissolution", DPST-79-269, February 26, 1979.

8. D. L. Kiser, "Removal of Salt from High-Level Waste Tanks by Density-Driven Circulation or Mechanical Agitation", DP-1587, January 1981.

9. G. H. Street, "Salt Removal from Tank 10", TA 2-959A, April 9, 1979.

10. G. H. Street, "Salt Removal from Tank 10", TA 2-959, March 31, 1980.

11. J. C. Bailey, "Tank 10 Salt Removal Demonstration Details", DPSP-79-17-11, April 11, 1979.

12. C. J. Thomas, "Salt Removal from Tank 10", TC-2-959, September 14, 1983.

13. Bill Parish, "Reducing or Eliminating the Use of Slurry Pumps from High Level Waste Tanks", July 19, 1993.

14. R. H. Perry and C. H. Chilton, Chemical Engineer's Handbook, 5th Ed., New York: McGraw-Hill, 1973.

15. K. Kwon, "Heat Balance an Thermal Analysis of Tank 30 \& Tank 29 as 3H Evaporator Concentrate Receipt Tanks", WSRC-TR-2001-00024, Rev. 0, March 2001.

16. K. C. Kwon, "Tank 48 and Tank 50 Liquid Phase Thermal Analysis for Cycle 1", WSRCTR-95-0220, Rev. 0, September 14, 1995.

17. K. C. Kwon, "Tank 41 Content Temperature During Salt Removal Operations", WSRC-TR95-0089, Rev. 0, March 9, 1995.

18. K. C. Kwon, "Tank 25F Mixed Waste Thermal Analysis During Salt Removal Batch Process With Cooling Coils Off', WSRC-TR-97-0067, Rev. 0, October 1, 1997. 


\section{Appendix 1}

Selected Sketches, Test Data and Example Calculations
A) Molecular Diffusion (or Horizontal Plane) Dissolution
B) Modified Density Gradient Dissolution
C) Mixing by Flygt Mixer(s) and Dissolution
D)Mixing by Slurry Pump and Dissolution 


\section{A) Molecular Diffusion Dissolution Without Agitation}

Molecular Diffusion - 25C IW (Case 1)

Horizontal Plane Dissolution of Salt by

Molecular Diffusion and Thermal Diffusion

Highly Concentrated or Saturated Salt Solution

Near Salt Cake-Liquid Interface

Low Concentration or Less Saturated Salt Solution

Near Vapor-Liquid Interface

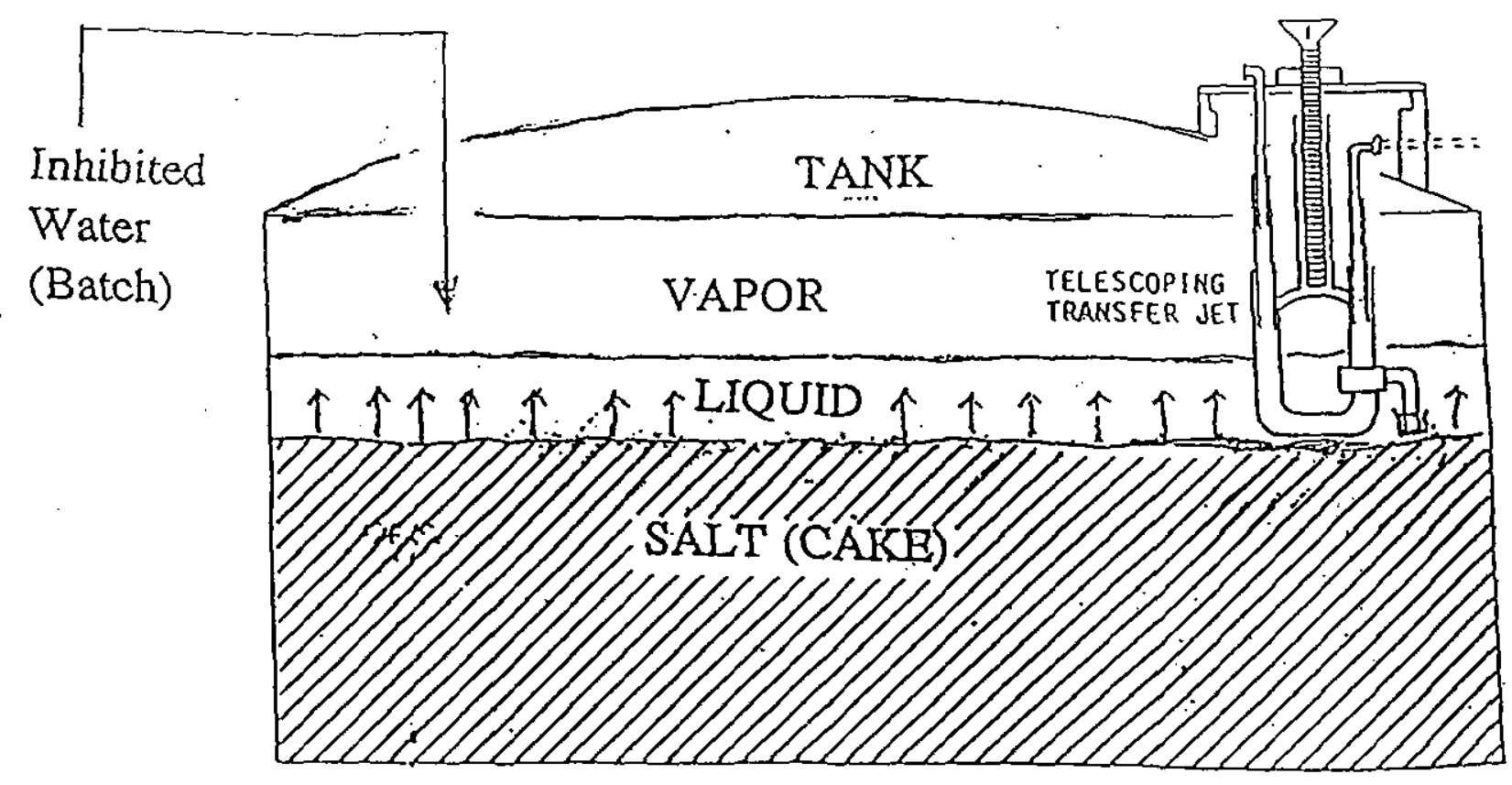

\begin{tabular}{|l|r|r|r|r|}
\hline salt dissolution rate $(\mathrm{gpm})$ & $\begin{array}{r}\text { Low here } \\
1.83\end{array}$ & 1.18 & $\begin{array}{r}\text { high } \\
2.47\end{array}$ & $\begin{array}{l}\text { Horizontal plane dissol. lab test } \\
1.18 \text { and diffusion calc. value }\end{array}$ \\
\hline tolal time (months) & 7.03. & 8.63 & 6.27 & 2.47 were shown as low \& high \\
\hline
\end{tabular}


Reference (A) - D. Kiser, Savannah River Laboratory (Jan/81), DP-1587, "Removal of Salt from High-Level Waste Tanks By Density Driven Circulation or Mechanical Agitation*

Horizontal Plane Dissolution

\begin{tabular}{|c|c|c|c|}
\hline Items \ Case & $\begin{array}{l}\text { Four Liter } \\
\text { Beaker Test }\end{array}$ & $\begin{array}{l}\text { Type IV } \\
\text { Tank } 22 \mathrm{H}\end{array}$ & $\begin{array}{l}\text { Type IIIa } \\
\text { Tank } 37 \mathrm{H}\end{array}$ \\
\hline $\begin{array}{l}\text { Tank ID (D) } \\
D \text { in meter } \\
\text { Diameter Ratio }\end{array}$ & $\begin{array}{rl}15 & \mathrm{~cm} \\
0 & \text { meter } \\
1 & \\
\end{array}$ & $\begin{array}{rl}85 & \mathrm{ft} \\
26 & \text { meters } \\
173.8791 & \\
\end{array}$ & $\begin{aligned} & 85 \mathrm{ft} \\
& 26 \text { meters } \\
& 173.8791 \\
&\end{aligned}$ \\
\hline $\begin{array}{l}\text { Area, (AI) } \\
\text { ID Area Ratio }\end{array}$ & $\begin{array}{r}0.0174 \mathrm{~m} 2 \\
1 \\
\end{array}$ & $\begin{array}{l}527.1784 \mathrm{~m} 2 \\
30233.97 \\
\end{array}$ & $\begin{array}{l}527.1784 \mathrm{~m} 2 \\
30233.97 \\
\end{array}$ \\
\hline $\begin{array}{l}\text { Center Cylinder OD } \\
\text { Center Column Area (A.2) }\end{array}$ & $\begin{array}{ll}0 & \mathrm{ft} \\
0 & \mathrm{~m} \\
0 & \mathrm{~m} 2\end{array}$ & $\begin{array}{ll}0 & \mathrm{ft} \\
0 & \mathrm{~m} \\
0 & \mathrm{~m} 2 \\
\end{array}$ & $\begin{array}{rl}6.75 & \mathrm{ft} \\
2.0574 & \mathrm{~m} \\
3.324507 \mathrm{~m} 2\end{array}$ \\
\hline $\begin{array}{l}\text { Net Area }(A=A 1-A 2) \\
\text { Equiv } D e=\left(4^{*} A e / p i\right) \wedge 0.5 \\
\text { Equiv. Dia. Ratio } \\
\text { Net Area Ratio } \\
\end{array}$ & $\begin{array}{rl}0.0174 & \mathrm{~m} 2 \\
0 & \text { meter } \\
1 & \\
1 & \\
\end{array}$ & \begin{tabular}{|rl}
$527.1784 \mathrm{~m} 2$ \\
25.908 meters \\
173.8791 & \\
30233.97 & \\
\end{tabular} & $\begin{array}{ll}523.8539 \mathrm{~m} 2 \\
25.82617 \text { meters } \\
173.3300 \\
30043.31 \\
\end{array}$ \\
\hline Cake Dissolution Rate & $\begin{array}{l}0.06 \mathrm{~cm} / \mathrm{hr} \\
0.000010 \mathrm{~m} 3 / \mathrm{hr} \\
0.002763 \mathrm{gal} / \mathrm{hr} \\
0.000046 \mathrm{gpm}\end{array}$ & $1.39 \mathrm{gpm}$ & $1.38 \mathrm{gpm}$ \\
\hline $\begin{array}{l}\text { salt dissolution ratio } \\
2.4\end{array}$ & $0.000039 \mathrm{gpm}$ & $1.19 \mathrm{gpm}$ & $1.18 \mathrm{gpm}$ \\
\hline 2.3 & $0.000040 \mathrm{gpm}$ & $1.22 \mathrm{gpm}$ & $1.21 \mathrm{gpm}$ \\
\hline 2.2 & $0.000041 \mathrm{gpm}$ & $1.26 \mathrm{gpm}$ & $1.25 \mathrm{gpm}$ \\
\hline 2.1 & $0.000043 \mathrm{gpm}$ & $1.30 \mathrm{gpm}$ & $1.29 \mathrm{gpm}$ \\
\hline 2.0 & $0.000044 \mathrm{gpm}$ & $1.34 \mathrm{gpm}$ & $1.34 \mathrm{gpm}$ \\
\hline 1.9 & $0.000046 \mathrm{gpm}$ & $1.39 \mathrm{gpm}$ & $1.38 \mathrm{gpm}$ \\
\hline $\begin{array}{l}\text { Salt Solution Removal(B) } \\
\mathrm{B} \text { in gpm } \\
\text { Salt Dissolution } \\
\text { Rate }=\mathrm{B} /(2.4+1)\end{array}$ & \begin{tabular}{|ll}
$\underline{0.505}$ & $\mathrm{ml} / \mathrm{m}$ \\
0.000133 & $\mathrm{gpm}$ \\
0.000039 & $\mathrm{gpm}$
\end{tabular} & $\begin{array}{rl}15.27 & \mathrm{~L} / \mathrm{min} \\
4.03 & \mathrm{gal} / \mathrm{min} \\
1.19 & \mathrm{gpm}\end{array}$ & $\begin{array}{rl}15.17 & \mathrm{~L} / \mathrm{min} \\
4.01 \mathrm{gal} / \mathrm{min} \\
1.18 \mathrm{gpm}\end{array}$ \\
\hline
\end{tabular}


Molecular Diffusion Case

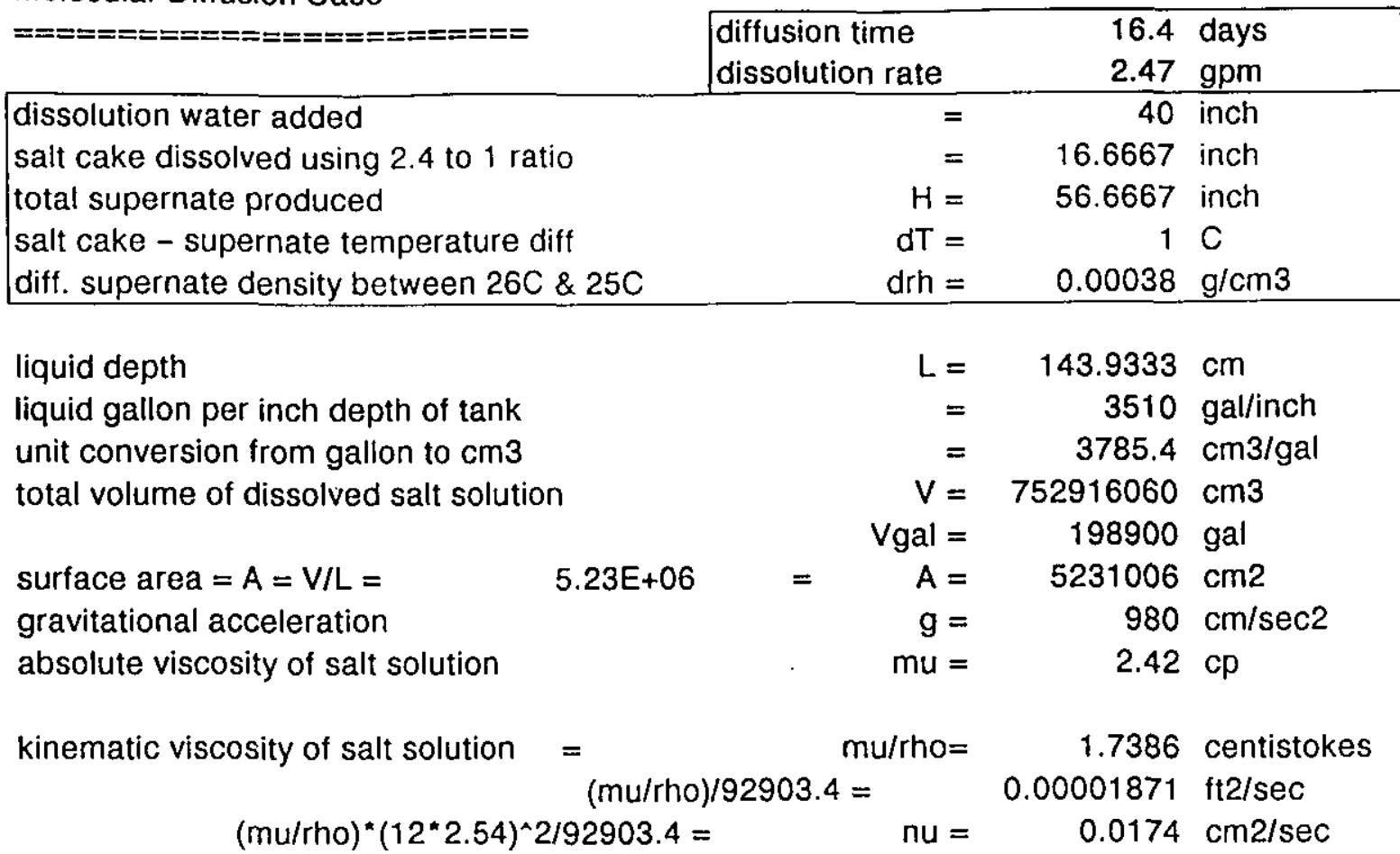

Grashof Number

$$
\mathrm{Gr}=(\mathrm{g})(\mathrm{L} \wedge 3)\left(\mathrm{drh}^{\prime}\right) /\left(\mathrm{rho}^{\star} n \mathrm{u}^{\wedge} 2\right)=\quad \mathrm{Gr}=2639213298
$$

Schmidt Number

kinematic visco. $/$ diffusivity $=\quad \mathrm{Sc}=\mathrm{nu} / \mathrm{Df}=\quad 1107.40$

Sherwood number

\begin{tabular}{|c|c|c|c|}
\hline $\begin{array}{l}S h=0.13 * G^{\wedge}(1 / 3) * S^{\wedge}(1 / 3)= \\
S h=(k) * L / D f\end{array}$ & $\mathrm{Sh}=$ & 1858.68 & \\
\hline Mass Transfer Coeff $=k=(S h) * D f / L=$ & $k=$ & 0.00020274 & $\mathrm{~cm} / \mathrm{sec}$ \\
\hline Mass Transfer Rate $=\mathrm{J}=\mathrm{k} * \mathrm{~A} * \mathrm{dC}$ & $J=$ & 207.81 & $\mathrm{~g} / \mathrm{sec}$ \\
\hline $\begin{array}{l}\text { total volume of dissolved salt solution } \\
\text { differential density between water \& salt solu. }= \\
\text { differential mass between water \& salt solu }=\mathrm{dM}=\end{array}$ & $V=$ & $\begin{array}{r}752916060 \\
0.392 \\
295067804\end{array}$ & $\begin{array}{l}\mathrm{cm} 3 \\
\text { g/cm3 } \\
\text { grams }\end{array}$ \\
\hline
\end{tabular}

total transfer time of differential mass

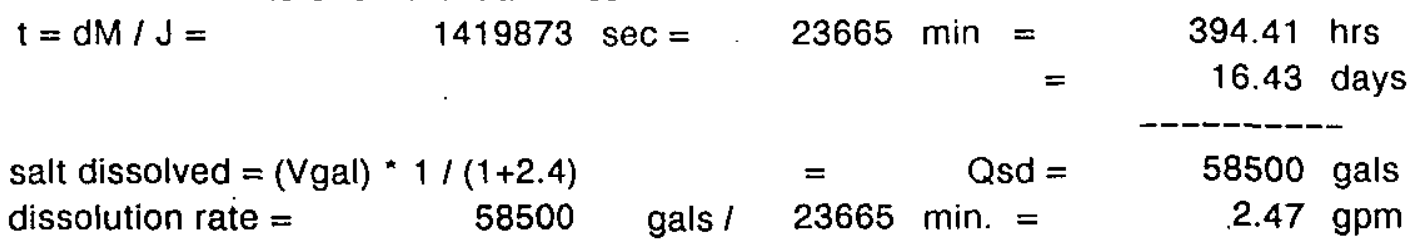




\section{Modified Density Gradient (MDG) Dissolution}

Low Density Inhibited Water or Supernate --- Upper Layer

High Density Dissolved Salt Solution --- Lower Layer or Well

Dissolved Salt Solution To Be Transferred Out During Dissolution

$$
\begin{aligned}
& \text { MDG - 25C IW (Case 2) } \\
& \text { MDG - 50C IW (Case 3) } \\
& \text { MDG - 75C IW (Case 4) }
\end{aligned}
$$

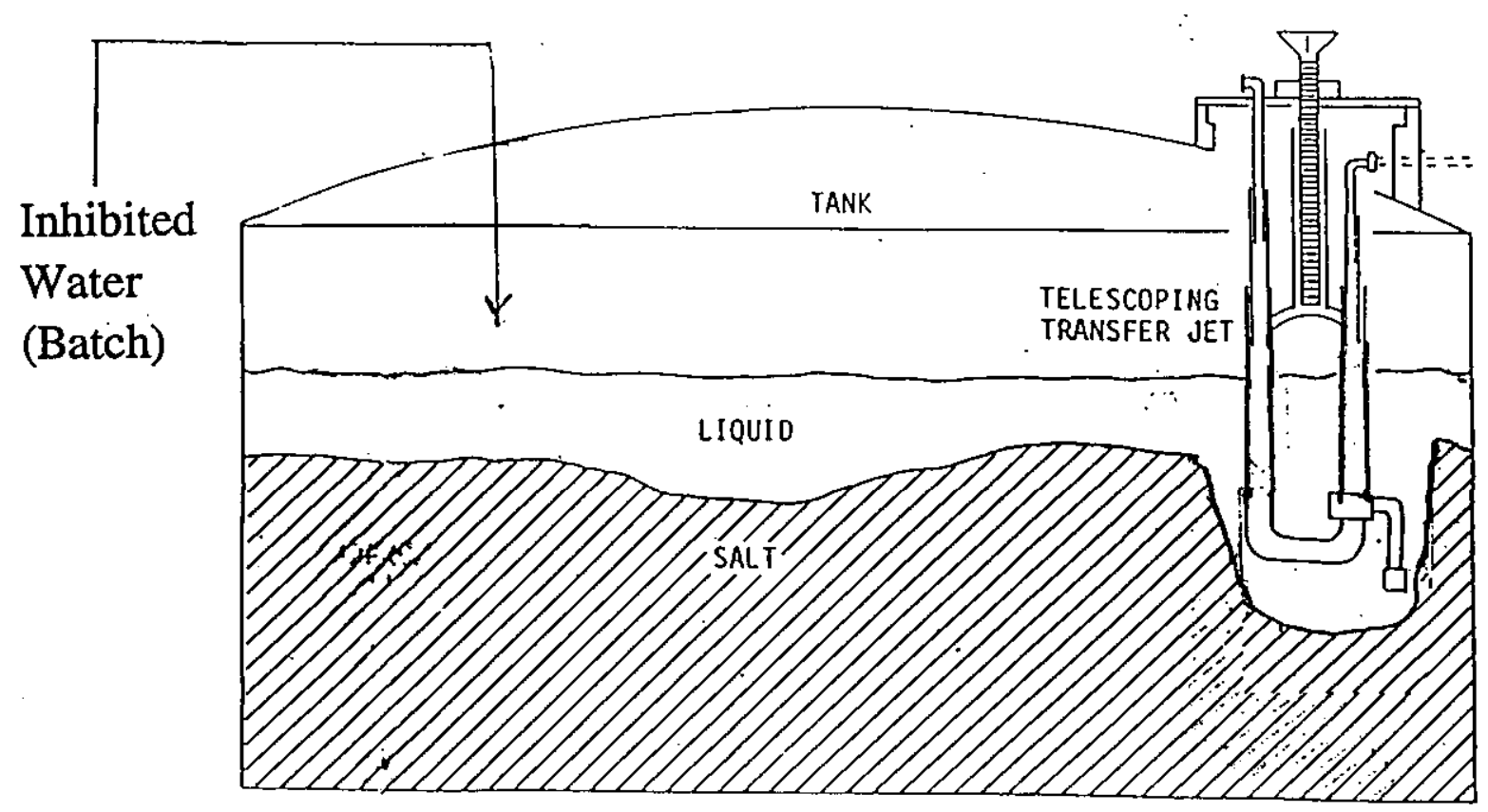


Reference (A) D. Kiser, Savannah River Laboratory (Jan/81), DP-1587, "Removal of Salt from High-Level Waste Tanks By Density Driven Circulation or Mechanical Agitation"

Reference (B) B. Wiersma, SRTC (Aug/96), WSRC-TR-96-0160,

"An Investigation of Density Driven Salt Dissolution Techniques"

Density-Driven Circulation Test

\begin{tabular}{|c|c|c|c|c|c|c|}
\hline \multirow{2}{*}{$\begin{array}{l}\text { Items } \mid \text { Case } \\
\text { Trough Size or ID } \\
\text { Equiv circle ID in meter } \\
\text { Equiv. Diameter Ratio }\end{array}$} & \multirow{2}{*}{$\begin{array}{l}\begin{array}{l}1981 \text { Lab Jar } \\
\text { Test (A) }\end{array} \\
\begin{array}{rl}31 & \mathrm{~cm} \\
0 & \text { meter } \\
1\end{array}\end{array}$} & \multirow{2}{*}{$\begin{array}{l}1996 \text { Trough } \\
\text { Test (B) } \\
3 * \times 36^{n} \times 15^{\text {"high }} \\
0.2979 \mathrm{~m} \\
0.9766\end{array}$} & \multicolumn{2}{|c|}{$\begin{array}{l}\text { Type IV } \\
\text { Tank 22H }\end{array}$} & \multicolumn{2}{|c|}{$\begin{array}{l}\text { Type IIIa } \\
\text { Tank 37H }\end{array}$} \\
\hline & & & $\begin{array}{r}85 \\
26 \\
84.94 \\
\end{array}$ & $\begin{array}{l}\mathrm{ft} \\
\text { meters }\end{array}$ & $\begin{array}{r}85 \\
26 \\
84.94 \\
\end{array}$ & $\begin{array}{l}\mathrm{ft} \\
\text { meters }\end{array}$ \\
\hline $\begin{array}{l}\text { Area (A1) } \\
\text { Area Ratio }\end{array}$ & $\begin{array}{r}0.0731 \mathrm{~m} 2 \\
1 \\
\end{array}$ & $\begin{array}{l}0.0697 \mathrm{~m} 2 \\
0.9537 \\
\end{array}$ & $\begin{array}{l}527.1784 \\
7215.527 \\
\end{array}$ & & $\begin{array}{r}527.18 \\
.7216 \\
\end{array}$ & $\mathrm{~m} 2$ \\
\hline $\begin{array}{l}\text { Center Cỵlinder OD } \\
\text { Center Column Area (A2) }\end{array}$ & $\begin{array}{ll}0 & \mathrm{ft} \\
0 & \mathrm{~m} \\
0 & \mathrm{~m} 2 \\
\end{array}$ & $\begin{array}{ll}0 & \mathrm{ft} \\
0 & \mathrm{~m} \\
0 & \mathrm{~m} 2 \\
\end{array}$ & $\begin{array}{l}0 \\
0 \\
0\end{array}$ & $\begin{array}{l}\mathrm{ft} \\
\mathrm{m} \\
\mathrm{m} 2 \\
\end{array}$ & $\begin{array}{r}7 \\
2 \\
3.32 \\
\end{array}$ & $\begin{array}{l}\mathrm{ft} \\
\mathrm{m} \\
\mathrm{m} 2\end{array}$ \\
\hline $\begin{array}{l}\text { Net Area if } A=(\mathrm{A} 1-\mathrm{A} 2) \\
\text { Equiv } \mathrm{De}=\left(4^{*} \mathrm{Ae} / \mathrm{pi}\right)^{\wedge} 0.5 \\
\text { Equiv: Dia. Ratio } \\
\text { Net Area Ratio }\end{array}$ & $\begin{array}{rll}0.0731 & \mathrm{~m} 2 \\
0 & \text { meter } \\
1 & \\
1 & \\
\end{array}$ & $\begin{array}{l}0.0697 \mathrm{~m} 2 \\
0.2979 \text { meter } \\
0.9766 \\
0.9537\end{array}$ & $\begin{array}{r}527.1784 \\
25.908 \\
84.94426 \\
7216 \\
\end{array}$ & $\begin{array}{l}\mathrm{m} 2 \\
\text { meters }\end{array}$ & $\begin{array}{r}523.85 \\
25.83 \\
84.68 \\
7170 \\
\end{array}$ & $\begin{array}{l}\mathrm{m} 2 \\
\text { meters }\end{array}$ \\
\hline Cake Dissolu & $\begin{array}{ll}\underline{0.31} \mathrm{~cm} / \mathrm{h} \\
0.00023 \mathrm{~m} 3 / \mathrm{hr} \\
0.05983 \mathrm{gal} / \mathrm{hr}\end{array}$ & $\begin{array}{r}0.25 \mathrm{~cm} / \mathrm{hr} \\
0.00018 \mathrm{~m} 3 / \mathrm{hr} \\
0.04662 \cdot \mathrm{gal} / \mathrm{hr}\end{array}$ & $\begin{array}{l}\text { Salt Cak } \\
(\mathrm{gpm}) \mathrm{b} \\
\text { (A) }\end{array}$ & $\begin{array}{l}\text { e Dis } \\
\text { y usi } \\
\text { (B) }\end{array}$ & $\begin{array}{l}\text { lution } \\
\text { test d } \\
\text { (A) }\end{array}$ & $\begin{array}{l}\text { Rate } \\
\text { ata } \\
\text { (B) } \\
\end{array}$ \\
\hline salt $\mathrm{di}$ & $\begin{array}{c}0.00100 \mathrm{gpm} \\
\text { SD Ratio= }=1.913\end{array}$ & $\begin{array}{c}0.00078 \mathrm{gpm} \\
\mathrm{SD} \cdot \text { Ratio= }=2.4\end{array}$ & 7.20 & 5.88 & 7.15 & 5.84 \\
\hline 2.4 & $0.00085 \mathrm{gpm}$ & $0.00078 \mathrm{gpm}$ & 6.17 & $\underline{5.88}$ & 6.13 & $\underline{5.84}$ \\
\hline 2.3 & $0.00088 \mathrm{gpm}$ & $0.00080 \mathrm{gpm}$ & 6.35 & 6.06 & 6.31 & 6.02 \\
\hline 2. & $0.00091 \mathrm{gpm}$ & $0.00083 \mathrm{gpm}$ & 6.55 & 6.25 & 6.51 & 6.21 \\
\hline 2.1 & $0.00094 \mathrm{gpm}$ & $0.00085 \mathrm{gpm}$ & 6.76 & 6.45 & 6.72 & 6.41 \\
\hline 2.0 & $0.00097 \mathrm{gpm}$ & $0.00088 \mathrm{gpm}$ & 6.99 & 6.66 & 6.95 & 6.62 \\
\hline 1.913 & $0.00100 \mathrm{gpm}$ & $0.00091 \mathrm{gpm}$ & $\underline{7.20}$ & 6.86 & $\underline{7.15}$ & 6.82 \\
\hline $\begin{array}{l}\text { Salt Solution Removal(B) } \\
\mathrm{B} \text { in gpm }\end{array}$ & $\frac{11}{\mathrm{ml} / \mathrm{min}}$ & $\begin{array}{rl}10 & \mathrm{ml} / \mathrm{min} \\
0.00264 & \mathrm{gpm}\end{array}$ & 20.97 & 19.99 & 20.84 & 19.86 \\
\hline $\begin{array}{l}\text { Salt Dissolution } \\
\text { Rate }=B /(2.4+1)\end{array}$ & $0.00085 \mathrm{gpm}$ & $0.00078 \mathrm{gpm}$ & $\begin{array}{l}6.17 \\
\mathrm{gpm} \\
\end{array}$ & $\begin{array}{l}5.88 \\
\mathrm{gpm}\end{array}$ & $\begin{array}{l}6.13 \\
\mathrm{gpm}\end{array}$ & $\begin{array}{l}5.84 \\
\text { gpm } \\
\end{array}$ \\
\hline & & & (salt dissol & 1. atio & 4 case) & \\
\hline
\end{tabular}




\section{Modified Density Gradient with 25C Inhibited Water (Batch 1)}

a) initial salt cake level (average of $262^{\prime \prime} \& 277^{n}$ ) -

b) inhibited water batch $=\sim 80 \%$ of $10 \mathrm{Kgal}$ tank -.......-. inhibited water pumping to TK $37(80$ to $120 \mathrm{gpm})$ total time of 1 batch IW addition to TK $37=$ IWt IW addition -..- 1 batch per $12 \mathrm{hr}$ shift \& total IW add hr needed/net pumping $\mathrm{hr}=\mathrm{IWt} / \mathrm{IWp}=12 / 1.33 \ldots$ equiv. continuous IW addition rate $=I W t / \mathrm{IWp}_{\mathrm{p}}=100 \mathrm{gpm} / 9--$ batch water add hrs $=(40 \mathrm{in} * 3510 \mathrm{gal} / \mathrm{in}) /(11.11 \mathrm{gpm} * 60 \mathrm{~min} / \mathrm{hr})=$

c) jet transfer TK 37->TK 30 (Existing Decant Before Batch)

d) salt dissolution rate $(\mathrm{gpm})$

\begin{tabular}{|l|r|r|r|}
\cline { 2 - 4 } transfer to TK $26(\mathrm{gpm})$ & 3.55 & 1.25 & 5.84 \\
\hline total time (months) & 12.05 & 4.25 & 19.86 \\
\hline
\end{tabular}

269.50 inch

8000 gallons per batch

$100 \mathrm{gpm} ; \mathrm{IWp}=1.33 \mathrm{hrs}$

12 hrs

2 batches per day

9

$11.11 \mathrm{gpm}$

210.6 hrs $=8.8$ days

60 gpm (not Batch Case)

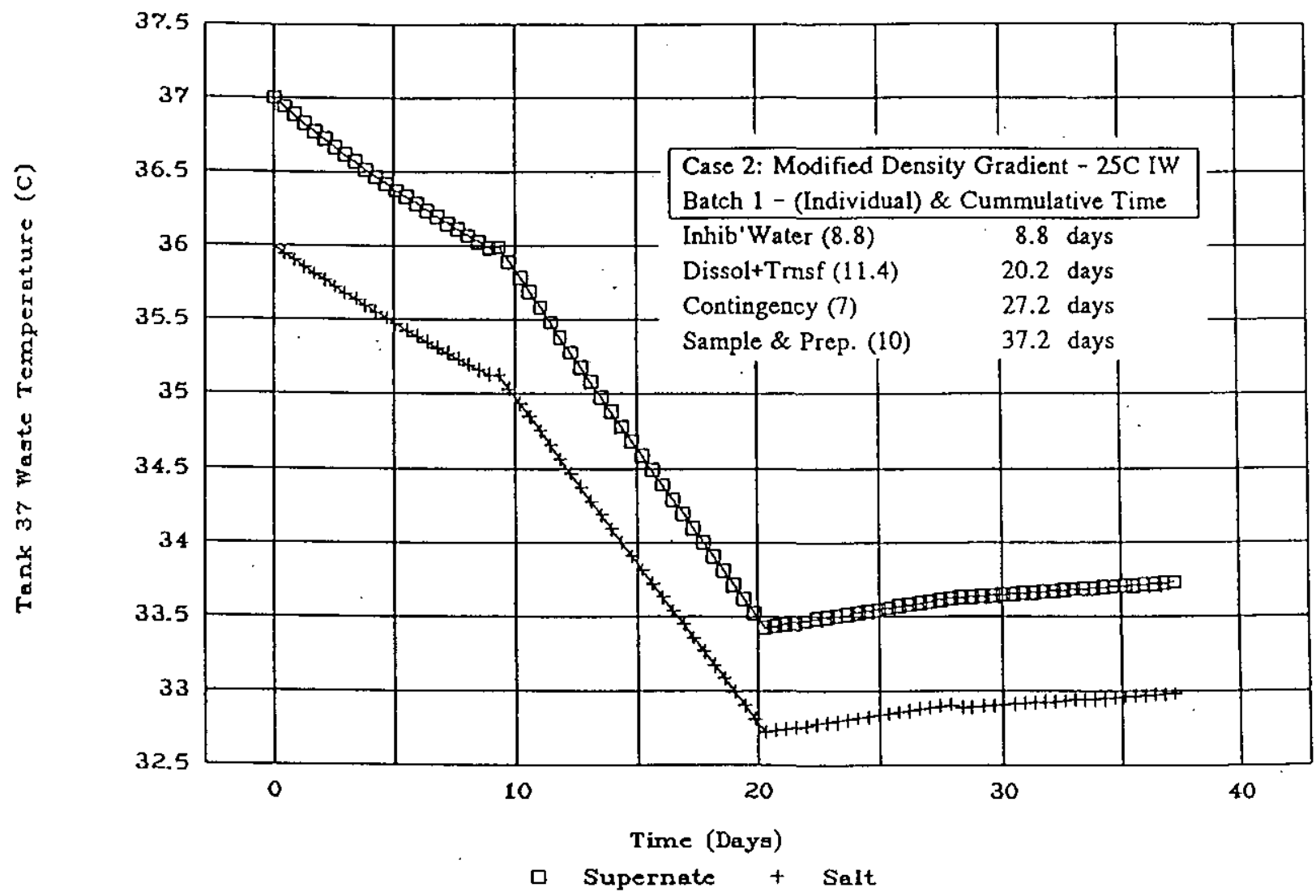

\begin{tabular}{|c|c|c|c|c|c|c|c|c|}
\hline $\begin{array}{r}\text { Batch \#1 } \\
\therefore \\
\end{array}$ & $\begin{array}{c}\text { starting } \\
\text { level }\end{array}$ & $\begin{array}{l}\text { water } \\
\text { added }\end{array}$ & $\begin{array}{c}\text { salt } \\
\text { dissolved }\end{array}$ & $\begin{array}{c}\text { total } \\
\text { supernate }\end{array}$ & $\begin{array}{c}\text { crystallized } \\
\text { salt cake }\end{array}$ & $\begin{array}{c}\text { total } \\
\text { content }\end{array}$ & $\begin{array}{l}\text { Inhib'Water } \\
\text { Disso+Trnsf }\end{array}$ & $\begin{array}{l}210.6 \\
275.0\end{array}$ \\
\hline depth (in) & 271.50 & 40.0 & 16.67 & 58.67 & 254.83 & 313.5 & \multirow[t]{2}{*}{ Contingency } & \multirow[t]{2}{*}{168.0} \\
\hline gallons & 952965 & 140400 & 58500 & 205920 & 894465 & 1100385 & & \\
\hline$\%$ & $86.6 \%$ & $12.8 \%$ & $5.3 \%$ & $18.7 \%$ & $81.3 \%$ & $100.0 \%$ & Sampl\&Prep. & 240.0 \\
\hline \multicolumn{2}{|l|}{ net hours } & 210.6 & 275.0 & 275.0 & transfer & batch total & (days) & 37.2 \\
\hline
\end{tabular}




\section{Modified Density Gradient with 25C Inhibited Water (Batch 2)}

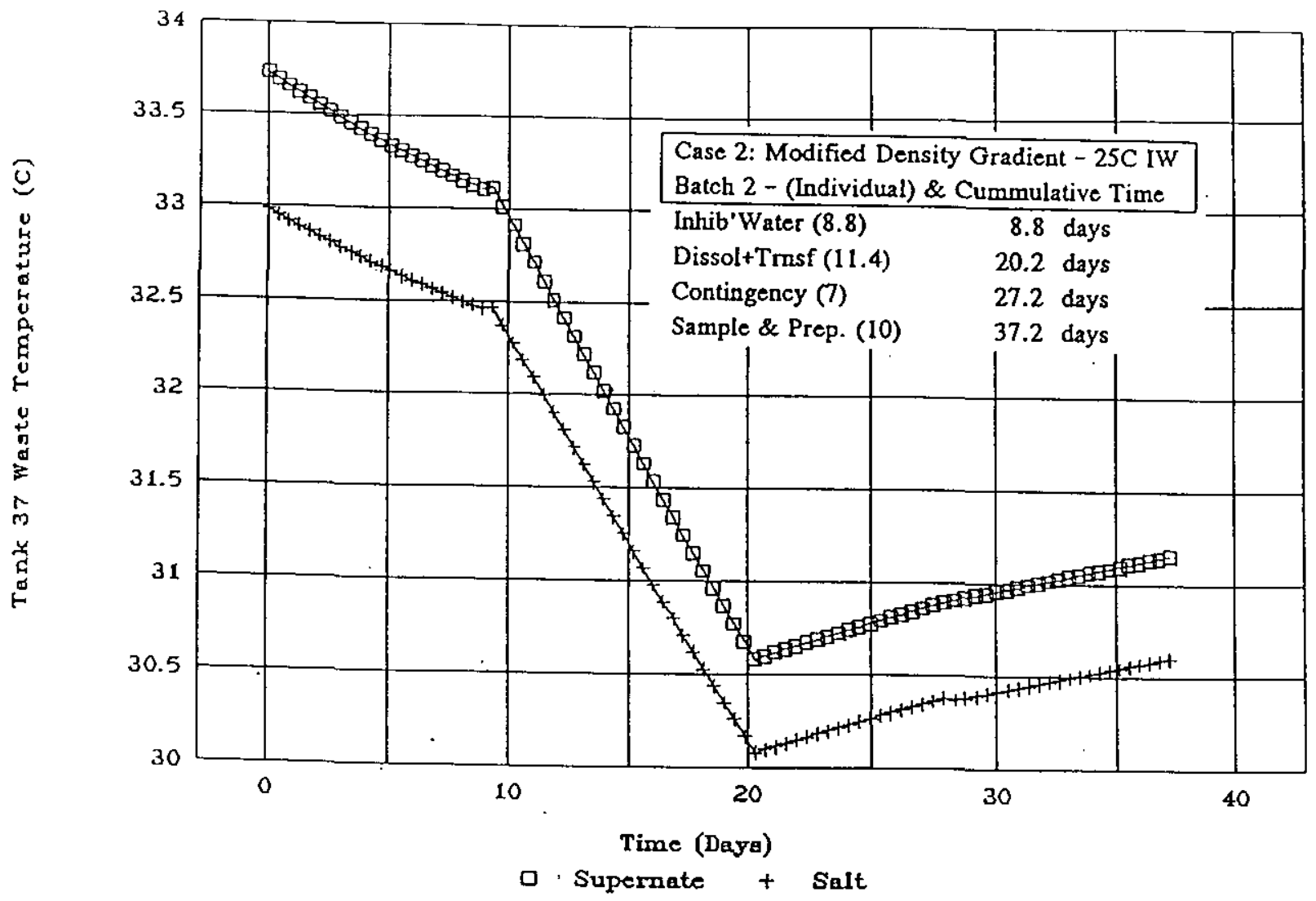

\begin{tabular}{|c|c|c|c|c|c|c|c|c|}
\hline Batch $\# 2$ & starting & water & salt & total & crystallized & total & Inhib'Water & 210.6 \\
\hline depth (in) & & & dissolved & supernate & salt cake & content & Disso+Trnsf & 275.0 \\
\hline depth (in) & 256.83 & 40.0 & 16.67 & 58.67 & 240.17 & 298.8 & \multirow[t]{2}{*}{ Contingency } & \multirow[t]{2}{*}{168.0} \\
\hline gallons & 901485 & 140400 & 58500 & 205920 & 842985 & 1048905 & & \\
\hline$\%$ & $85.9 \%$ & $13.4 \%$ & $5.6 \%$ & $19.6 \%$ & $80.4 \%$ & $100.0 \%$ & Sampl\&Prep. & 240.0 \\
\hline net hours & & 210.6 & 275.0 & 275.0 & transfer & batch total & 1 (days) & 37.2 \\
\hline
\end{tabular}




\section{Modified Density Gradient with 25C Inhibited Water (Batch 3)}

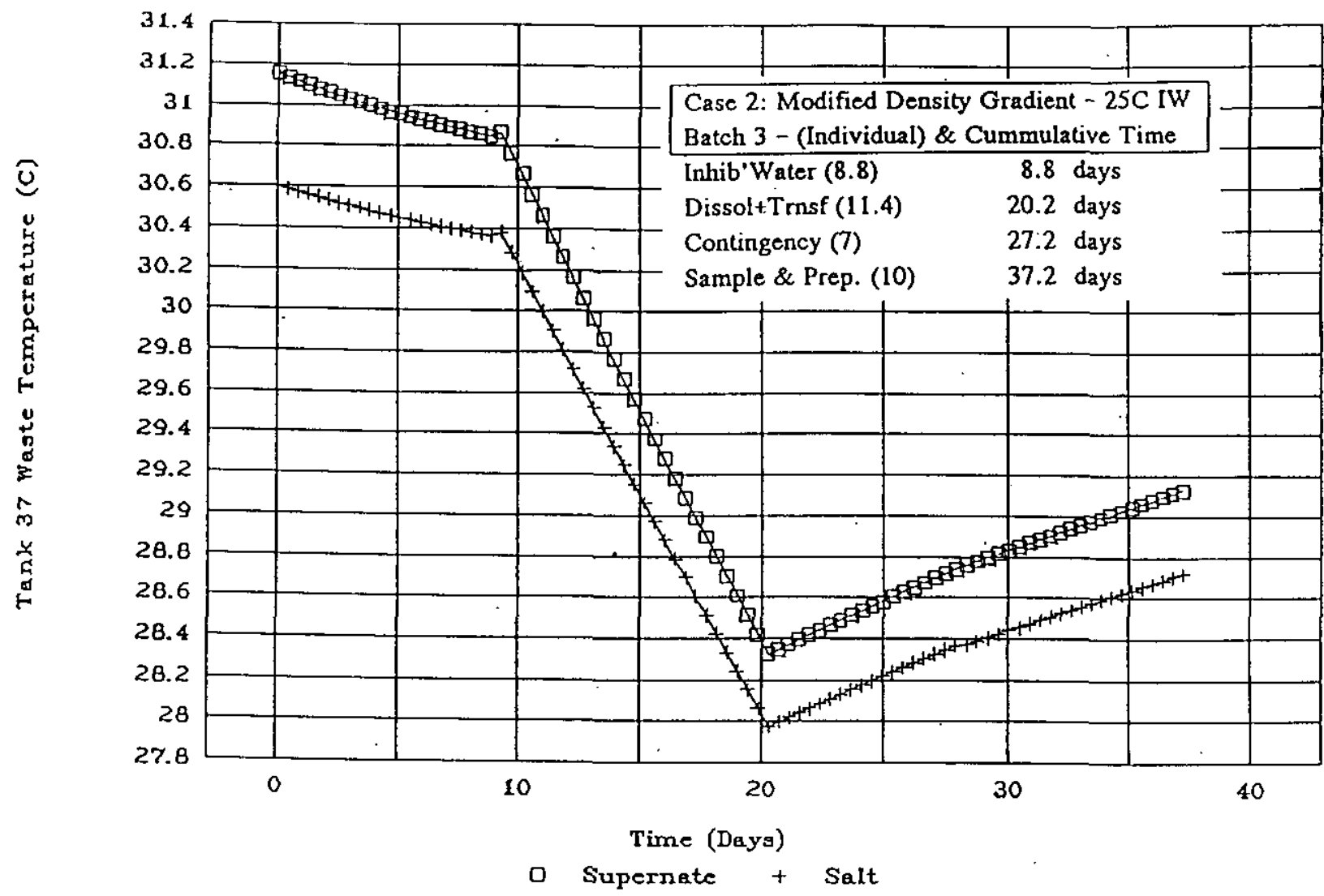

\begin{tabular}{|c|c|c|c|c|c|c|c|c|}
\hline$\frac{\text { Batch \#3 }}{}$ & $\begin{array}{c}\text { starting } \\
\text { level }\end{array}$ & $\begin{array}{l}\text { water } \\
\text { added }\end{array}$ & $\begin{array}{c}\text { salt } \\
\text { disol }\end{array}$ & $\begin{array}{c}\text { total } \\
\text { supernate }\end{array}$ & $\begin{array}{l}\text { crystallized } \\
\text { salt cake }\end{array}$ & $\begin{array}{c}\text { total } \\
\text { content }\end{array}$ & Inhib'Water & $\begin{array}{l}210.6 \\
275.0\end{array}$ \\
\hline depth (in) & 242.17 & 40.0 & 16.67 & 58.67 & 225.50 & 284.2 & \multirow{2}{*}{ Contingency } & \multirow{2}{*}{168.0} \\
\hline gallons & 850005 & 140400 & 58500 & 205920 & 791505 & 997425 & & \\
\hline$\%$ & $85.2 \%$ & $14.1 \%$ & $5.9 \%$ & $20.6 \%$ & $79.4 \%$ & $100.0 \%$ & Sampl\&Prep. & 240.0 \\
\hline net hours & & 210.6 & 275.0 & 275.0 & transfer & batch total & 1 (days) & 37.2 \\
\hline
\end{tabular}




\section{Modified Density Gradient with 25C Inhibited Water (Batch 4)}

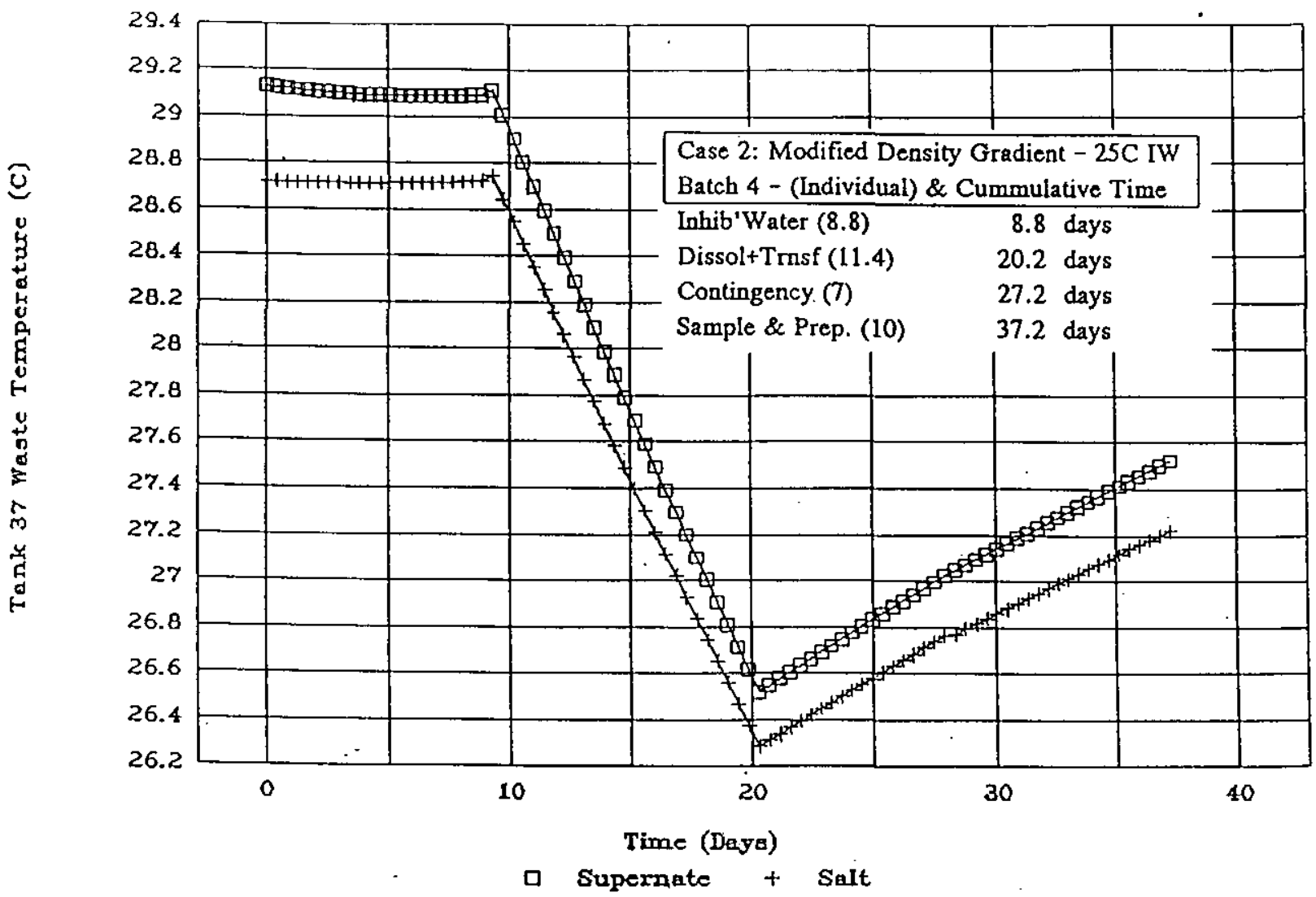

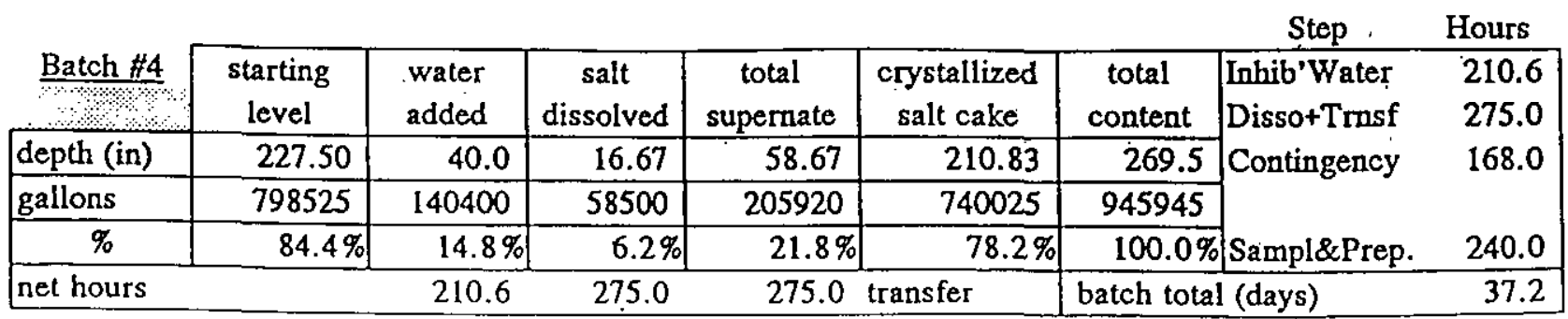


C) Mixing by Mechanical Agitation (Flygt Mixer)

1 Mixer 25C IW (Case 5)

1 Mixer 50C IW (Case 7)

1 Mixer 75C IW (Case 10)

3 Mixers 25C IW (Case 9)

3 Mixers 50C IW (Case 13)

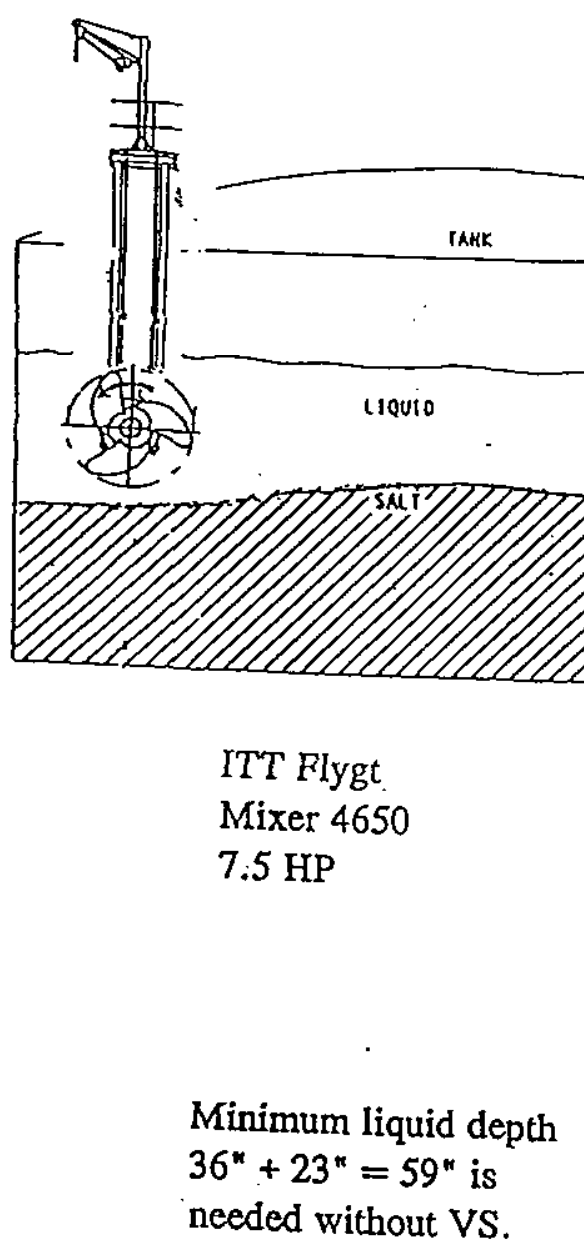

2 Mixers 25C IW (Case 6)

2 Mixers 50C IW (Case 11)

2 Mixers 75C IW (Case 14)

3 Mixers 75C IW (case 16)

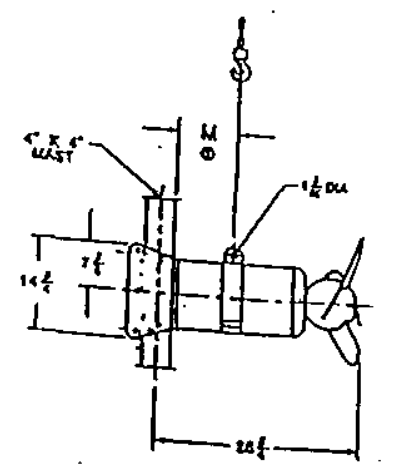

Vortex Suppressor
(VS)

When used with VS only $1 \mathrm{ft}$ submergence is needed.

Minimum liquid depth $12^{n}+23^{n}=35^{n}$ is needed with VS.
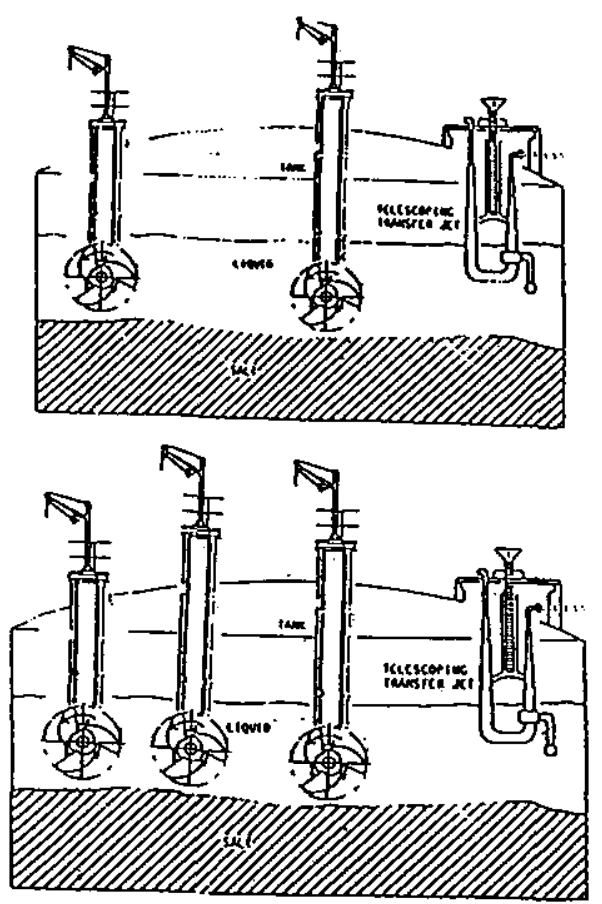

Minimum

Submergence

\section{2 " with VS}

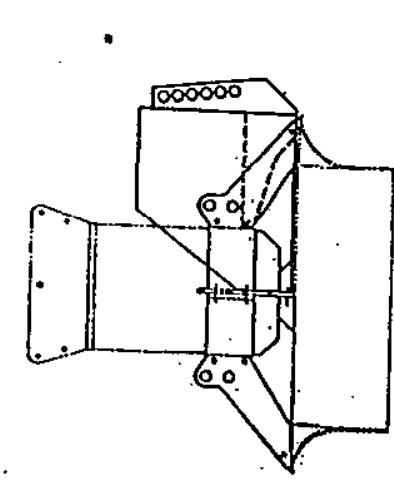




\section{One Flygt Mixer with 25C Inhibited Water}

a) initial salt cake level (average of $262^{\prime \prime} \& 277^{\prime \prime}$ )

b) inhibited water batch $=\sim 80 \%$ of $10 \mathrm{Kgal} \mathrm{tank}$ inhibited water pumping to TK 37 (80 to $120 \mathrm{gpm}$ ) total time of 1 batch IW addition to TK $37=$ IWt

IW addition 1 batch per 12 hr shift \& tocal IW add hr needed/net pumping hr $=$ IWt $/ \mathrm{IWp}=12 / 1.33$ equiv. continuous IW addition rate $=I W t / I W p=100 \mathrm{gpm} / 9$ batch water add hrs $=(40 \mathrm{in} * 3510 \mathrm{gal} / \mathrm{in}) /(11.11 \mathrm{gpm} * 60 \mathrm{~min} / \mathrm{hr})=$

c) jet transfer TK 37->TK 30 and or TK 26

\begin{tabular}{|c|r|r|r|}
\cline { 2 - 4 } \multicolumn{1}{c|}{ d) salt dissolution rate (gpm) } & 3.00 & 2.00 & high \\
\hline total time (months) & 5.89 & 6.78 & 4.00 \\
\hline
\end{tabular}

\subsection{0 inch}

8000 gallons per batch

$100 \mathrm{gpm} ; \mathrm{IWp}=1.33 \mathrm{hrs}$

12 hrs

2 batches per day

9

$11.11 \mathrm{gpm}$

$210.6 \mathrm{hrs}=8.8$ days

60 gpm

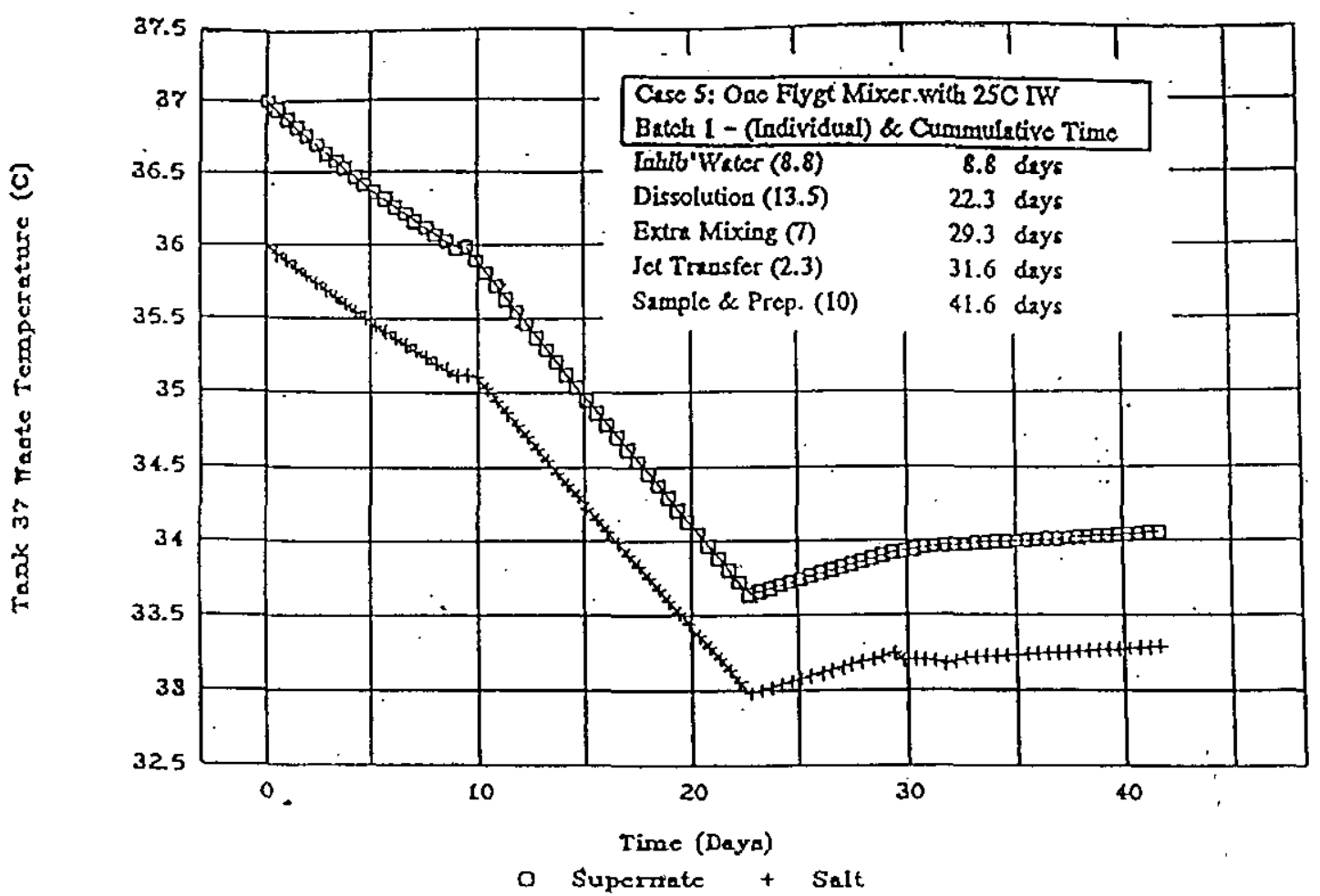

\begin{tabular}{|c|c|c|c|c|c|c|c|c|}
\hline \multirow{2}{*}{$\underline{\text { Batch \#1 }}$} & starting & water & salt & total & crystallized & total & Inhib'Water & 210.6 \\
\hline & level & added & dissolved & supernate & cake & content & Dissolut & 325.0 \\
\hline depth (in) & 271.50 & 40.0 & 16.67 & 58.67 & 254.83 & 313.5 & Contir & 168.0 \\
\hline gallons & 52965 & 40400 & 58500 & 205920 & 894465 & 1100385 & Insfer & 55.3 \\
\hline$\%$ & $6.6 \%$ & $12.8 \%$ & $5.3 \%$ & $18.7 \%$ & $81.3 \%$ & $100.0 \%$ & Sampl\&Prep. & 240.0 \\
\hline net hours & & 210.6 & 325.0 & 55.3 & transfer & batch total & (d & 41.6 \\
\hline
\end{tabular}


Case 7: One Flygt Mixer with 50C IW

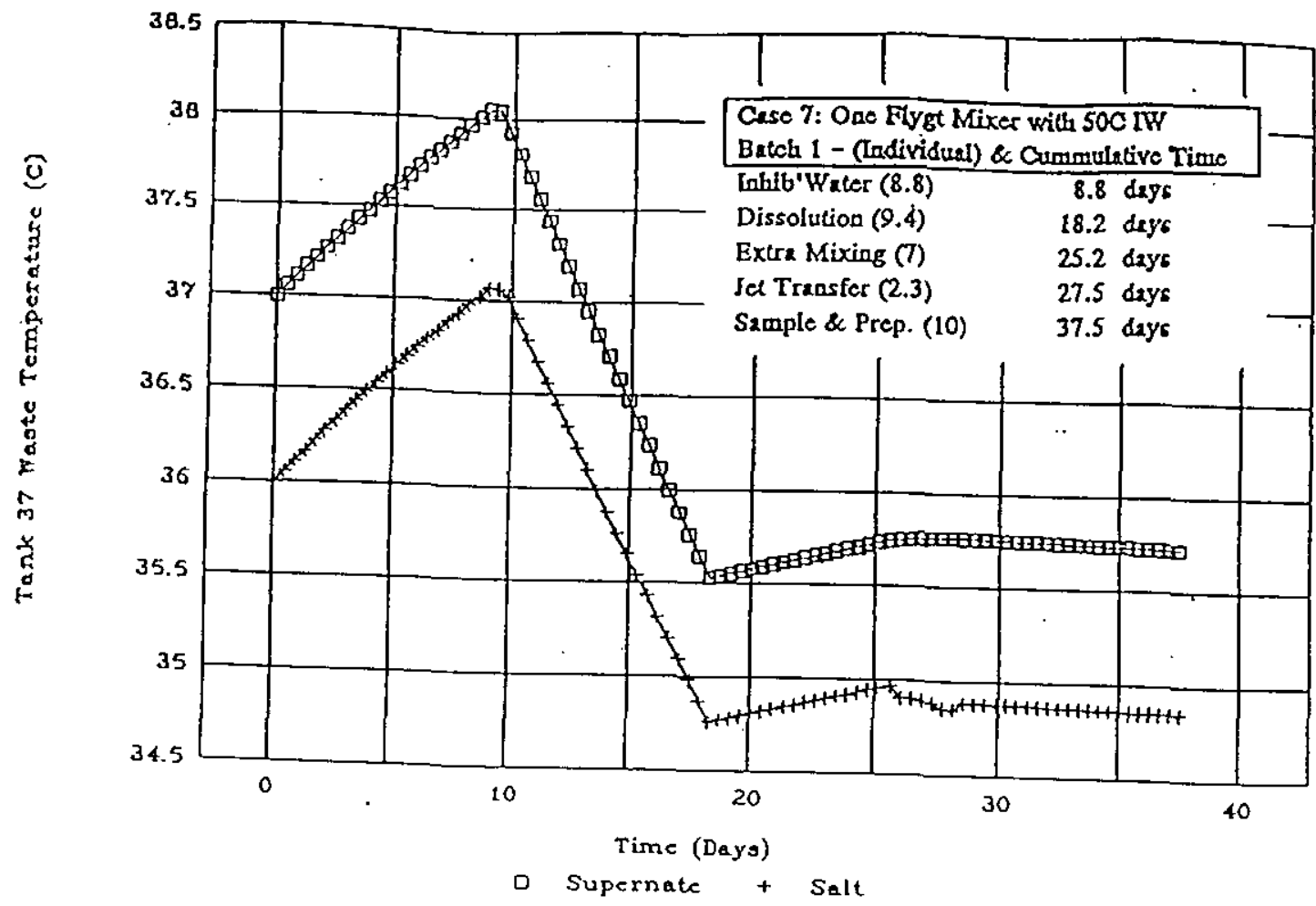

Case 10: One Flygt Mixer with 75C IW

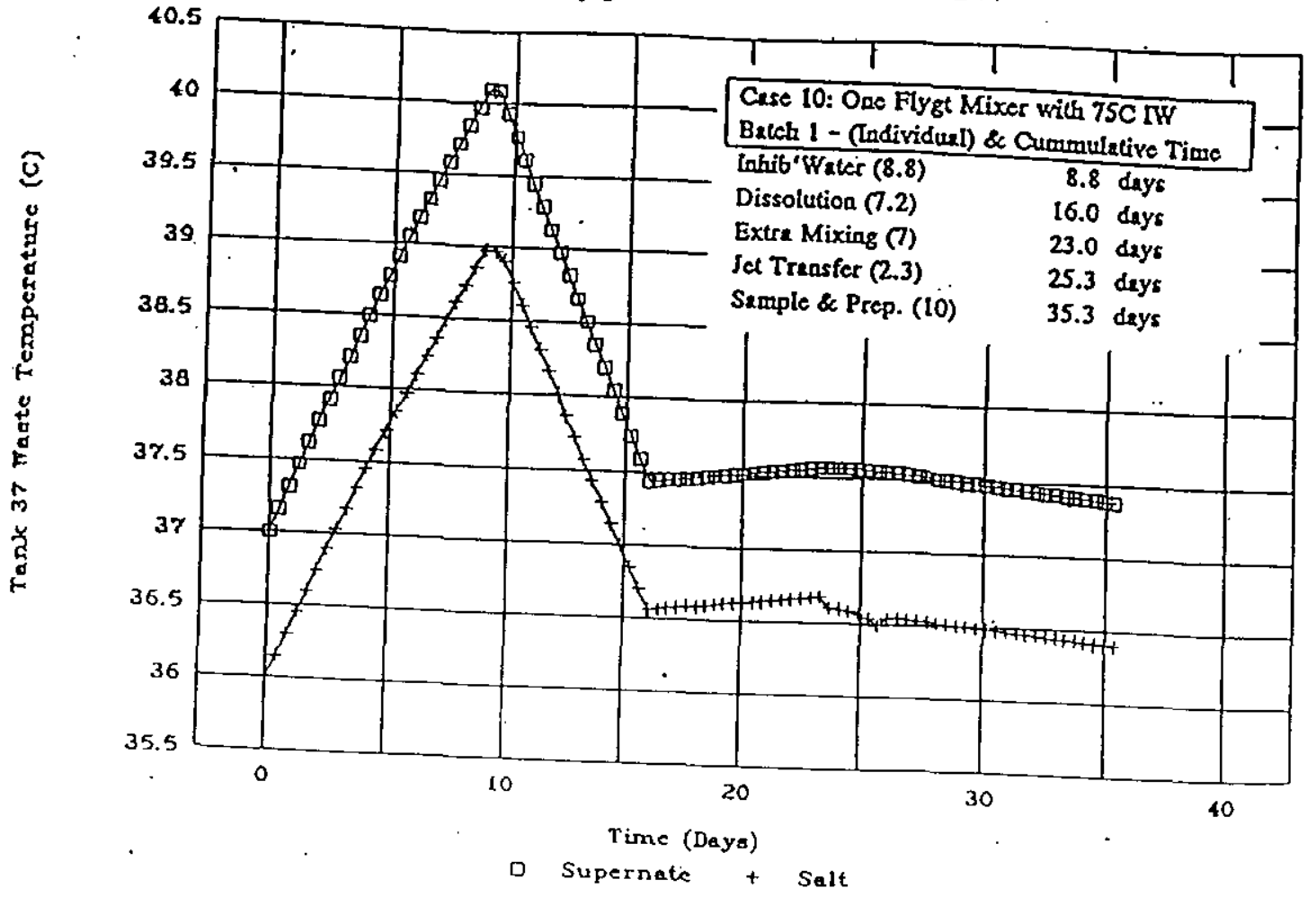




\title{
D) Mixing by Mechanical Agitation (Slurry Pump)
}

\author{
25C Inhibited Water (Case 8)
}

50C Inhibited Water (Case 12)

75C Inhibited Water (Case 15)

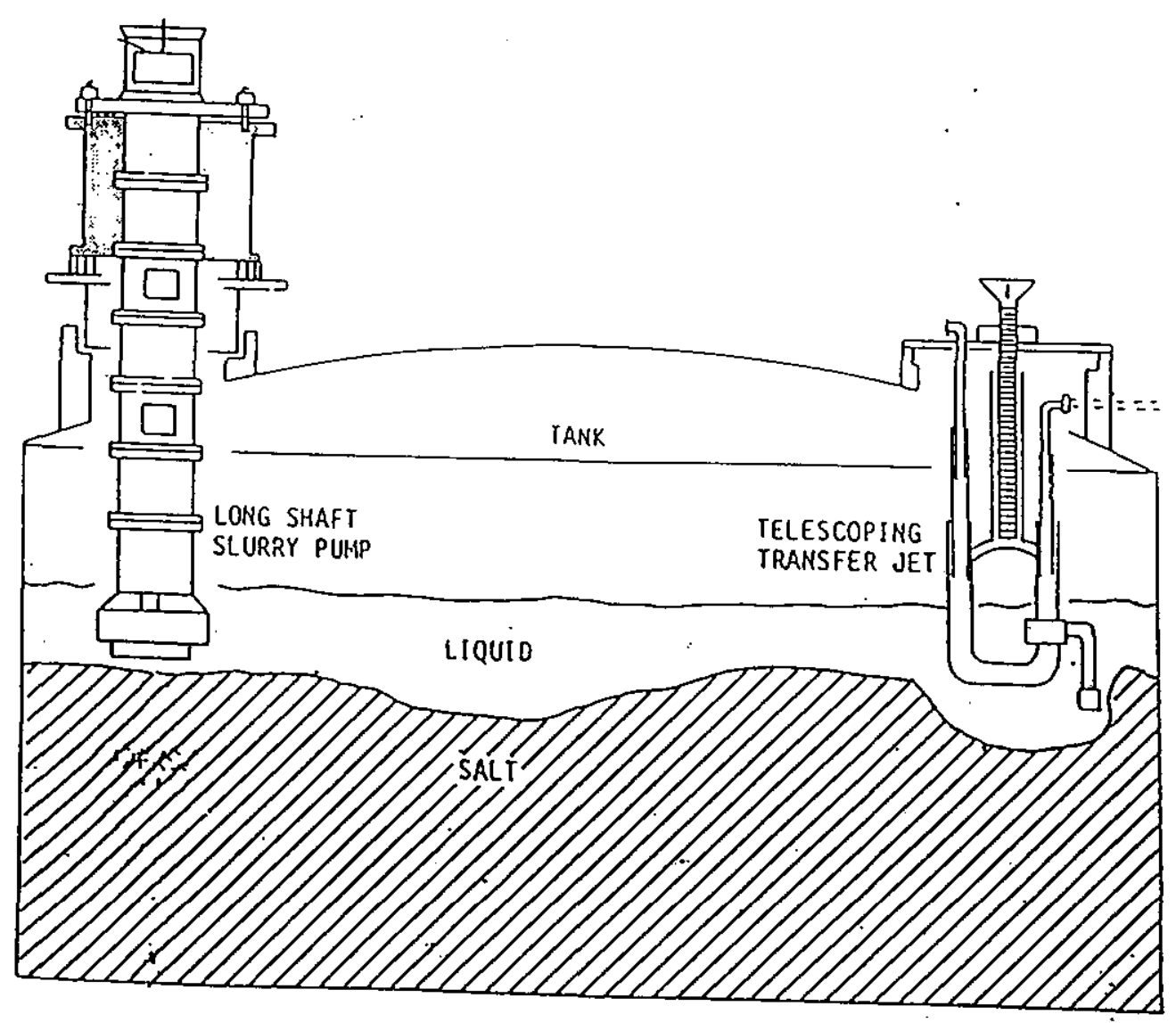

\section{Minimum Submergence}

16 inch above slurry pump bottom 


\section{One Slurry Pump with 25C Ambient Inhibited Water}

a) initial salt cake level (average of $262^{*} \& 277^{*}$ ) $=$

b) inhibited water batch $=\sim 80 \%$ of $10 \mathrm{Kgal}$ tank $=$

269.50 inch inhibited water pumping to TK 37 (80 to $120 \mathrm{gpm})=$ net pumping time of one batch IW to TK $37=$ IWp $=$ number of IW batches per $12 \mathrm{br}$-shift $=$ number of IW batches per day = total time of 1 batch IW addition to TK $37=$ IWt $=$ total IW time needed/net pumping time $=$ IWt/IWp $=$ 8000 gallons

100 gpm

$1.3 \mathrm{hrs}$

1 batch per shift

2 batches per day

$12 \mathrm{hrs}$

9

5 gpm

c) salt dissolution rate $=$

$60 \mathrm{gpm}$

d) jet transfer rate from TK 37 to TK 30 or TK $26=$ (60 to $100 \mathrm{gpm}$ )

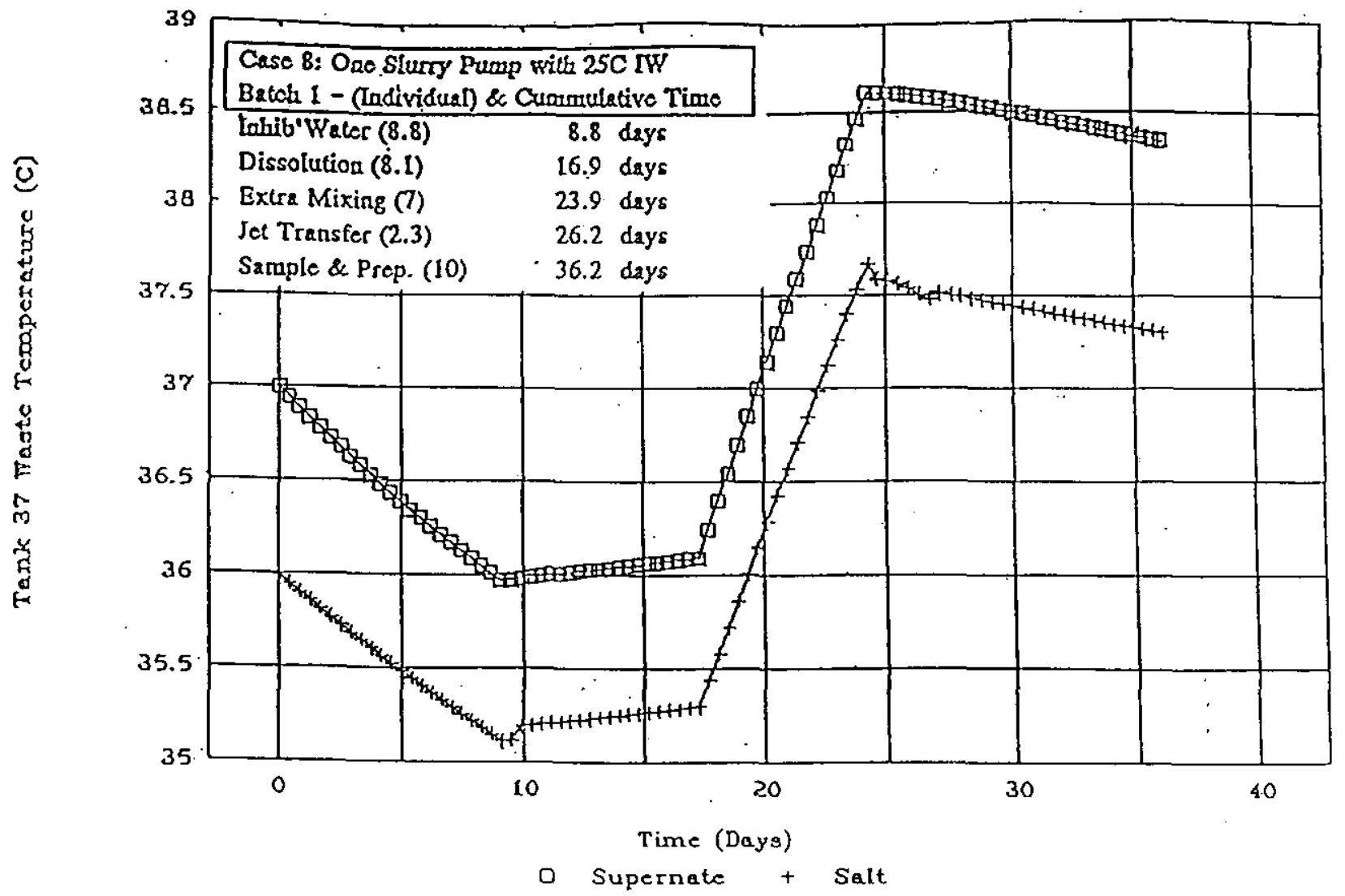

\begin{tabular}{|c|c|c|c|c|c|c|c|c|}
\hline Batch \#1 & $\begin{array}{c}\text { starting } \\
\text { level }\end{array}$ & $\begin{array}{l}\text { water } \\
\text { added }\end{array}$ & $\begin{array}{c}\text { salt } \\
\text { dissolved }\end{array}$ & $\begin{array}{c}\text { total } \\
\text { supernate }\end{array}$ & $\begin{array}{c}\text { crystallized } \\
\text { salt cake }\end{array}$ & $\begin{array}{c}\text { total } \\
\text { content }\end{array}$ & $\begin{array}{l}\text { Inhib'Water } \\
\text { Dissolution }\end{array}$ & $\begin{array}{l}210.6 \\
195.0\end{array}$ \\
\hline depth (in) & 271.50 & 40.0 & 16.67 & 58.67 & 254.83 & 313.5 & ExtraMixing & 168.0 \\
\hline gallons & 952965 & 140400 & 58500 & 205920 & 894465 & 1100385 & Jet Transfer & 55.3 \\
\hline$\%$ & $86.6 \%$ & $12.8 \%$ & $5.3 \%$ & $18.7 \%$ & $81.3 \%$ & $100.0 \%$ & Sampl\&Prep. & 240.0 \\
\hline net hours & & 210.6 & 195.0 & 55.3 & transfer & batch total & 1 (days) & 36.2 \\
\hline
\end{tabular}




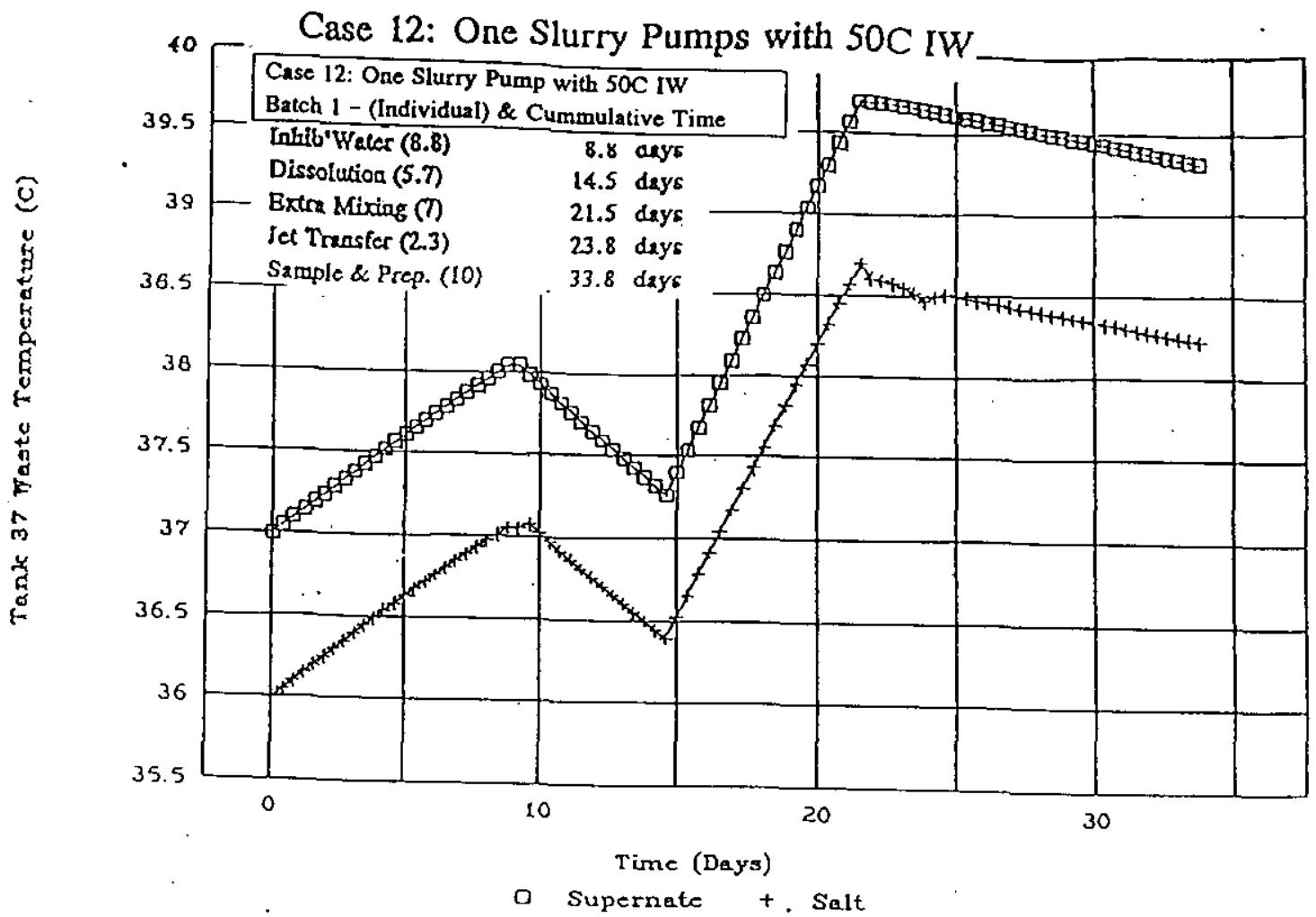

Case 15 One Slurry Pump with $75 \mathrm{C}$ IW

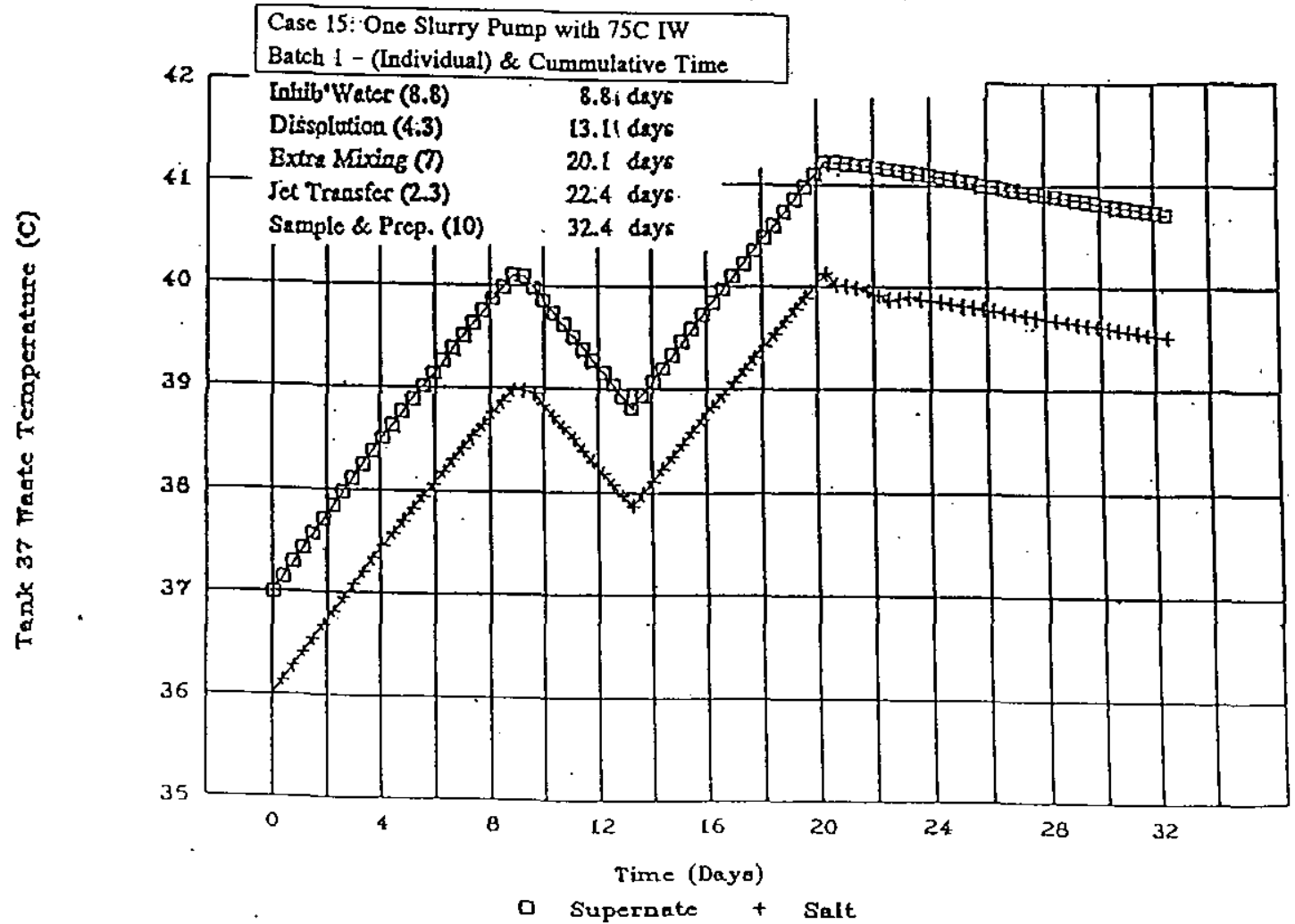




\section{Appendix 2 \\ Dissolution Calculations}

\begin{tabular}{|l|c|c|c|c|c|}
\hline Dissolution Method & $\begin{array}{c}\text { Dissolution } \\
\text { Rate }(\mathrm{gpm})\end{array}$ & $\begin{array}{c}\text { Dis. Rate } \\
\text { used }(\mathrm{gpm})\end{array}$ & $\begin{array}{c}\text { Dissolution } \\
\text { Time (days) }\end{array}$ & $\begin{array}{c}\text { Dissolution Time } \\
\text { \% of Total) }\end{array}$ & $\begin{array}{c}\text { Total Process } \\
\text { Time (months) }\end{array}$ \\
\hline 1. No Agitation $\left(25^{\circ} \mathrm{C}\right)$ & $1.18-2.47$ & 1.83 & 117 & 54 & 7.0 \\
\hline 2. MDG $\left(25^{\circ} \mathrm{C}\right)$ & $1.25-5.84$ & 3.55 & 74 & 46 & 5.3 \\
\hline 3. MDG $\left(50^{\circ} \mathrm{C}\right)$ & $1.78-8.32$ & 5.05 & 60 & 41 & 4.9 \\
\hline 4. MDG $\left(75^{\circ} \mathrm{C}\right)$ & $2.33-10.80$ & 6.56 & 53 & 37 & 4.6 \\
\hline 5. One Flygt Mixer $\left(25^{\circ} \mathrm{C}\right)$ & $2.0-4.0$ & 3.0 & 82 & 46 & 5.9 \\
\hline 6. Two Flygt Mixers $\left(25^{\circ} \mathrm{C}\right)$ & $2.8-5.7$ & 4.2 & 67 & 41 & 5.4 \\
\hline 7. One Flygt Mixer $\left(50^{\circ} \mathrm{C}\right)$ & $2.8-5.8$ & 4.3 & 66 & 40 & 5.4 \\
\hline 8. One Slurry Pump $\left(25^{\circ} \mathrm{C}\right)$ & $3.0-7.0$ & 5.0 & 61 & 38 & 5.2 \\
\hline 9. Three Flygt Mixers $\left(25^{\circ} \mathrm{C}\right)$ & $3.5-6.9$ & 5.2 & 59 & 38 & 5.1 \\
\hline 10. One Flygt mixer $\left(75^{\circ} \mathrm{C}\right)$ & $3.7-7.4$ & 5.6 & 57 & 37 & 5.1 \\
\hline 11. Two Flygt Mixers $\left(50^{\circ} \mathrm{C}\right)$ & $4.0-8.0$ & 6.1 & 55 & 36 & 5.0 \\
\hline 12. One Slurry Pump $\left(50^{\circ} \mathrm{C}\right)$ & $4.3-9.9$ & 7.1 & 51 & 34 & 4.9 \\
\hline 13. Three Flygt Mixers $\left(50^{\circ} \mathrm{C}\right)$ & $4.9-9.8$ & 7.4 & 50 & 34 & 4.8 \\
\hline 14. Two Flygt Mixers $\left(75^{\circ} \mathrm{C}\right)$ & $5.3-10.5$ & 8.6 & 47 & 33 & 4.7 \\
\hline 15. One Slurry Pump $\left(75^{\circ} \mathrm{C}\right)$ & $5.6-13.0$ & 9.3 & 46 & 32 & 4.7 \\
\hline 16. Three Flygt Mixers $\left(75^{\circ} \mathrm{C}\right)$ & $6.4-12.9$ & 10.6 & 43 & 31 & 4.6 \\
\hline
\end{tabular}




\section{TANK 37H DISSOLUTION CYCLE ANALYSIS}

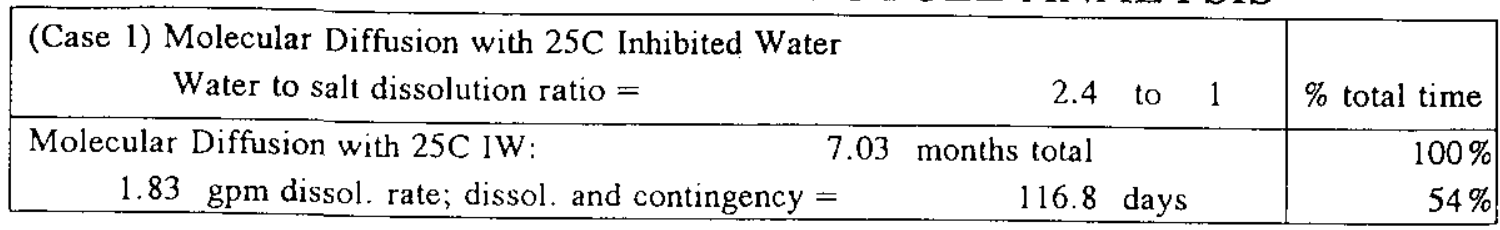

a) initial salt cake level (average of $262^{\prime \prime} \& 277^{\prime \prime}$ ) -- 269.50 inch

b) inhibited water batch $=\sim 80 \%$ of $10 \mathrm{Kgal}$ tank--------------

inhibited water pumping to TK 37 (80 to $120 \mathrm{gpm}$ ) ----------

total time of 1 batch IW addition to TK $37=$ IWt

1 batch per $12 \mathrm{hr}$ shift \&

total IW add $\mathrm{hr}$ needed/net pumping $\mathrm{hr}=\mathrm{IWt} / \mathrm{IWp}=12 / 1.33$

equiv. continuous IW addition rate $=\mathrm{IWt} / \mathrm{IWp}=100 \mathrm{gpm} / 9$----

batch water add hrs $=(40 \mathrm{in} * 3510 \mathrm{gal} / \mathrm{in}) /(11.11 \mathrm{gpm} * 60 \mathrm{~min} / \mathrm{hr})=$

c) jet transfer TK 37->TK 30 and or TK 26

$$
\begin{array}{rl}
8000 & \text { gallons per batch } \\
100 & \mathrm{gpm} ; \mathrm{IWp}=1.33 \mathrm{hrs} \\
12 & \mathrm{hrs} \\
2 & \text { batches per day } \\
9 &
\end{array}
$$

$11.11 \mathrm{gpm}$

\begin{tabular}{|c|c|c|c|c|c|c|c|c|}
\hline \multicolumn{7}{|c|}{ Molecular Diffusion with 25C IW: } & \multicolumn{2}{|r|}{ Hours } \\
\hline Decant & salt cake & water & salt & total liq or & crystallized & total & Inhib'Water & 0.0 \\
\hline Existing & + dissolv. & existing & dissolved & supernate & salt cake & content & Dissolution & 0.0 \\
\hline depth (in) & 293.53 & 57.66 & 24.03 & 81.69 & 269.50 & 351.0 & Contingency & 0.0 \\
\hline gallons & 1030278 & 202399 & 84333 & 286732 & 945945 & 1232010 & Jet Transfer & 77.7 \\
\hline$\%$ & $83.6 \%$ & $16.4 \%$ & $6.8 \%$ & $23.3 \%$ & $76.8 \%$ & $100.0 \%$ & Sampl\&Prep. & 240.0 \\
\hline net hours & & 0.0 & 0.0 & 77.7 & transfer & batch tota & (days) & 13.2 \\
\hline
\end{tabular}

210.6 hrs $=8.8$ days

\begin{tabular}{|c|c|c|c|c|c|c|c|c|}
\hline$\underline{\text { Batch \#1 }}$ & $\begin{array}{c}\text { starting } \\
\text { level }\end{array}$ & $\begin{array}{l}\text { water } \\
\text { added }\end{array}$ & $\begin{array}{c}\text { salt } \\
\text { dissolved }\end{array}$ & $\begin{array}{c}\text { total } \\
\text { supernate }\end{array}$ & $\begin{array}{l}\text { crystallized } \\
\text { salt cake }\end{array}$ & $\begin{array}{c}\text { total } \\
\text { content }\end{array}$ & $\begin{array}{l}\text { Inhib'Water } \\
\text { Dissolution }\end{array}$ & $\begin{array}{l}210.6 \\
532.8\end{array}$ \\
\hline depth (in) & 271.50 & 40.0 & 16.67 & 58.67 & 254.83 & 313.5 & Contingency & 168.0 \\
\hline gallons & 952965 & 40400 & 58500 & 205920 & 894465 & 1100385 & Jet Transfer & 55.3 \\
\hline$\%$ & $6 \%$ & $12.8 \%$ & $5.3 \%$ & $18.7 \%$ & $81.3 \%$ & $100.0 \%$ & Sampl\&Prep. & 240.0 \\
\hline net hours & & 210.6 & 532.8 & 55.3 & transfer & batch total & (days) & $50: 3$ \\
\hline
\end{tabular}

$60 \mathrm{gpm}$

\begin{tabular}{|c|c|c|c|c|c|c|c|c|}
\hline Batch \#2 & $\begin{array}{c}\text { starting } \\
\text { level }\end{array}$ & $\begin{array}{l}\text { water } \\
\text { added }\end{array}$ & $\begin{array}{c}\text { salt } \\
\text { dissolved }\end{array}$ & $\begin{array}{c}\text { total } \\
\text { supernate }\end{array}$ & $\begin{array}{c}\text { crystallized } \\
\text { salt cake }\end{array}$ & $\begin{array}{c}\text { total } \\
\text { content }\end{array}$ & $\begin{array}{l}\text { Inhib'Water } \\
\text { Dissolution }\end{array}$ & $\begin{array}{l}210.6 \\
532.8\end{array}$ \\
\hline depth (in) & 256.83 & 40.0 & 16.67 & 58.67 & 240.17 & 298.8 & Contingency & 168.0 \\
\hline gallons & 901485 & 140400 & 58500 & 205920 & 842985 & 1048905 & Jet Transfer & 55.3 \\
\hline$\%$ & $85.9 \%$ & $13.4 \%$ & $5.6 \%$ & $19.6 \%$ & $80.4 \%$ & $100.0 \%$ & Sampl\&Prep. & 240.0 \\
\hline \multicolumn{2}{|l|}{ net hours } & 210.6 & 532.8 & 55.3 & transfer & batch total & (days) & 50.3 \\
\hline
\end{tabular}

\begin{tabular}{|c|r|r|r|l|}
\cline { 2 - 5 } \multicolumn{1}{c|}{ d) salt dissolution rate (gpm) } & used here & low & high & Horizontal plane dissol. Iab test \\
\hline total time (months) & 7.83 & 1.18 & 2.47 & 1.18 and diffusion calc. value \\
\hline
\end{tabular}

\begin{tabular}{|c|c|c|c|c|c|c|c|c|}
\hline Batch \#3 & $\begin{array}{c}\text { starting } \\
\text { level }\end{array}$ & $\begin{array}{l}\text { water } \\
\text { added }\end{array}$ & $\begin{array}{c}\text { salt } \\
\text { dissolved }\end{array}$ & $\begin{array}{c}\text { total } \\
\text { supernate }\end{array}$ & $\begin{array}{c}\text { crystallized } \\
\text { salt cake }\end{array}$ & $\begin{array}{c}\text { total } \\
\text { content }\end{array}$ & $\begin{array}{l}\text { Inhib'Water } \\
\text { Dissolution }\end{array}$ & $\begin{array}{l}210.6 \\
532.8\end{array}$ \\
\hline depth (in) & 242.17 & 40.0 & 16.67 & 58.67 & 225.50 & 284.2 & Contingency & 168.0 \\
\hline gallons & 850005 & 140400 & 58500 & 205920 & 791505 & 997425. & Jet Transfer & 55.3 \\
\hline$\%$ & $85.2 \%$ & $14.1 \%$ & $5.9 \%$ & $20.6 \%$ & $79.4 \%$ & $100.0 \%$ & Sampl\&Prep. & 240.0 \\
\hline net hours & & 210.6 & 532.8 & \multicolumn{2}{|c|}{55.3 transfer } & \multicolumn{2}{|c|}{ batch total (days) } & 50.3 \\
\hline
\end{tabular}

Molecular Diffusion with 25C IW: 


\begin{tabular}{|c|c|c|c|c|c|c|c|c|}
\hline & & & & & & & Step & Hours \\
\hline Batch \#4 & $\begin{array}{c}\text { starting } \\
\text { level }\end{array}$ & $\begin{array}{l}\text { water } \\
\text { added }\end{array}$ & $\begin{array}{c}\text { salt } \\
\text { dissolved }\end{array}$ & $\begin{array}{c}\text { total } \\
\text { supernate }\end{array}$ & $\begin{array}{l}\text { crystallized } \\
\text { salt cake }\end{array}$ & $\begin{array}{c}\text { total } \\
\text { content }\end{array}$ & $\begin{array}{l}\text { Inhib'Water } \\
\text { Dissolution }\end{array}$ & $\begin{array}{l}210.6 \\
532.8\end{array}$ \\
\hline depth (in) & 227.50 & 40.0 & 16.67 & 58.67 & 210.83 & 269.5 & Contingency & 168.0 \\
\hline gallons & 798525 & 140400 & 58500 & 205920 & 740025 & 945945 & Jet Transfer & 55.3 \\
\hline$\%$ & $84.4 \%$ & $14.8 \%$ & $6.2 \%$ & $21.8 \%$ & $78.2 \%$ & $100.0 \%$ & Sampl\&Prep. & 240.0 \\
\hline net hours & & 210.6 & 532.8 & 55.3 & transfer & batch total & (days) & 50.3 \\
\hline
\end{tabular}

\section{(Case 1) Molecular Diffusion with 25C Inhibited Water}

Tank Level at the End of Batch $4=$

Salt Cake Level Reduced $=$

Total Inhibited Water Used=

dissolution \& transdfer time (a)

additional contingency time (b)

total dissolution, transf \& contingency $(a+b)=$

inhibited water addition $=$

initial TK decant transfer time $=$

test sample \& preparation=

total time $=$
$210.8+2=$

$$
269.5-210.8=
$$

561600 gallons $=$

2131.1 hrs $=$

$672.0 \mathrm{hrs}$

$842.4 \mathrm{hrs}=$

$298.7 \mathrm{hrs}=$ $1200.0 \mathrm{hrs}=$

$5144.2 \mathrm{hrs}=$
212.8 "TK Lvl

58.7 "TK Lvl

160.0 " TK Lvl

88.8 days

28.0 days

116.8 days

35.1 days

12.4 days

50.0 days

214.3 days

or 7.0 months

After the existing supernate transfer to TK 30 and the above four batch transfer to TK 26 , Tank 37 salt level of 212.8 inch will pass the project scope tank level of 227 inches.

Batch $\# 5$ is shown below even though it is not required to meet the original project scope level of 227 inches. The Tank 37 level after Batch 5 will be

198.2 inches if Batch 5 is processed.

\begin{tabular}{|c|c|c|c|c|c|c|c|c|}
\hline \multirow{3}{*}{ Batch \#5 } & \multirow{3}{*}{$\begin{array}{c}\text { starting } \\
\text { level }\end{array}$} & \multirow{3}{*}{$\begin{array}{l}\text { water } \\
\text { added }\end{array}$} & \multirow{3}{*}{\begin{tabular}{|c|} 
salt \\
dissolved
\end{tabular}} & \multirow{3}{*}{$\begin{array}{c}\text { total } \\
\text { supernate }\end{array}$} & \multirow{3}{*}{$\begin{array}{l}\text { crystallized } \\
\text { salt cake }\end{array}$} & \multirow{3}{*}{$\begin{array}{c}\text { total } \\
\text { content }\end{array}$} & Step & \multirow{2}{*}{$\frac{\text { Hours }}{210.6}$} \\
\hline & & & & & & & Inhib'Water & \\
\hline & & & & & & & Dissolution & 532.8 \\
\hline depth (in) & 212.83 & 40.0 & 16.67 & 58.67 & 196.17 & 254.8 & Contingency & 168.0 \\
\hline gallons & 747045 & 140400 & 58500 & 205920 & 688545 & 894465 & Jet Transfer & 55.3 \\
\hline$\%$ & $83.5 \%$ & $15.7 \%$ & $6.5 \%$ & $23.0 \%$ & $77.0 \%$ & $100.0 \%$ & Sampl\&Prep. & 240.0 \\
\hline net hours & & 210.6 & 532.8 & 55.3 & transfer & batch total & 1 (days) & 50.3 \\
\hline
\end{tabular}




\section{TANK 37H DISSOLUTION CYCLE ANALYSIS}

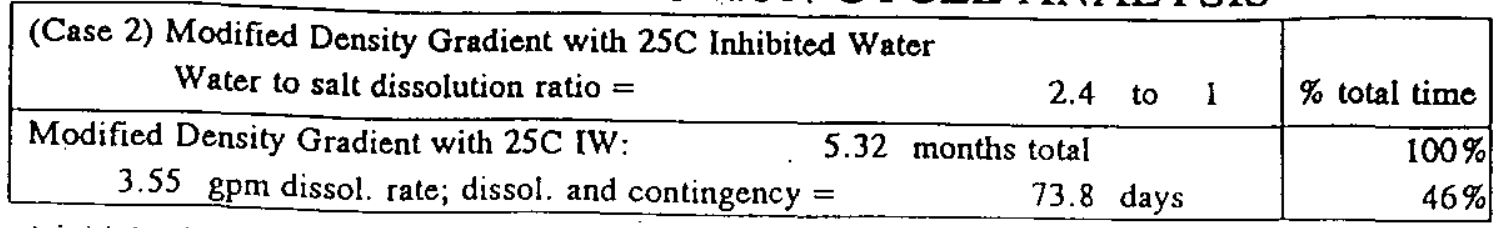

a) initial salt cake level (average of 262" \& 277") -- 269.50 inch

b) inhibited water batch $=\sim 80 \%$ of $10 \mathrm{Kgal}$ tank -.............-inhibited water pumping to TK 37 ( 80 to $120 \mathrm{gpm}$ ) total time of 1 batch IW addition to TK $37=$ IW $-2-2--$ IW addition---.-- 1 batch per $12 \mathrm{hr}$ shift \& total IW add hr needed/net pumping $\mathrm{hr}=\mathrm{IWt} / \mathrm{IWp}=12 / 1.33 \ldots \ldots$ equiv. continuous IW addition rate $=I W / I W p=100 \mathrm{gpm} / 9 \ldots \ldots$ batch water add $\mathrm{hrs}=(40 \mathrm{in} * 3510 \mathrm{gal} / \mathrm{in}) /(11.11 \mathrm{gpm} * 60 \mathrm{~min} / \mathrm{hr})=$ c) jet transfer TK 37->TK 30 (Existing Decant Before Batch) d) salt dissolution rate ( $\mathrm{gpm})$

\begin{tabular}{|l|r|r|r|l|}
\hline transfer to TK 26 (gpm) & 3.55 & 1.25 & 5.84 & $\begin{array}{l}\text { Avg of } 25 \% \text { slurry pump rate } \\
\text { and } 1979 \text { TK } 10 \text { data gives }\end{array}$ \\
\cline { 1 - 4 } total time (months) & 12.05 & 4.25 & 19.86 & and \\
\hline
\end{tabular}

Modified Density Gradient with 25C IW:

\begin{tabular}{|c|c|c|c|c|c|c|c|c|}
\hline \multicolumn{7}{|c|}{ Modified Density Gradient with $25 \mathrm{C}$ IW: } & \multicolumn{2}{|r|}{ Hours } \\
\hline Decant & salt cake & water & salt & total liq or & crystallized & total & Inhib'Water & 0.0 \\
\hline Existing & + dissolv. & existing & dissolved & supernate & salt cake & content & Dissolution & 0.0 \\
\hline depth (in) & 293.53 & 57.66 & 24.03 & 81.69 & 269.50 & 351.0 & Contingency & 0.0 \\
\hline gallons & 1030278 & 202399 & 84333 & 286732 & 945945 & 1232010 & Jet Transfer & 77.7 \\
\hline$\%$ & $83.6 \%$ & $16.4 \%$ & $6.8 \%$ & $23.3 \%$ & $76.8 \%$ & $100.0 \%$ & Sampl\&Prep. & 240.0 \\
\hline net hours & & 0.0 & 0.0 & 77.7 & transfer & batch total & (days) & 13.2 \\
\hline
\end{tabular}

\begin{tabular}{|c|c|c|c|c|c|c|c|c|}
\hline Batch \#1 & $\begin{array}{c}\text { starting } \\
\text { level }\end{array}$ & $\begin{array}{l}\text { water } \\
\text { added }\end{array}$ & $\begin{array}{c}\text { salt } \\
\text { dissolved }\end{array}$ & $\begin{array}{c}\text { total } \\
\text { supernate }\end{array}$ & $\begin{array}{c}\text { crystallized } \\
\text { salt cake }\end{array}$ & $\begin{array}{c}\text { total } \\
\text { content }\end{array}$ & $\begin{array}{l}\text { Inhib'Water } \\
\text { Disso+Trnsf }\end{array}$ & $\begin{array}{l}210.6 \\
275.0\end{array}$ \\
\hline depth (in) & 271.50 & 40.0 & 16.67 & 58.67 & 254.83 & 313.5 & \multirow[t]{2}{*}{ Contingency } & \multirow[t]{2}{*}{168.0} \\
\hline gallons & 952965 & 140400 & 58500 & 205920 & 894465 & 1100385 & & \\
\hline$\%$ & $5.6 \%$ & $12.8 \%$ & $.3 \%$ & $18.7 \%$ & $81.3 \%$ & $100.0 \%$ & Sampl\&Prep. & 240.0 \\
\hline hours & & 210.6 & 275.0 & $275.0 \mathrm{t}$ & transfer & batch total & (days) & 37.2 \\
\hline
\end{tabular}

\begin{tabular}{|c|c|c|c|c|c|c|c|c|}
\hline Batch & $\begin{array}{c}\text { starting } \\
\text { level }\end{array}$ & $\begin{array}{l}\text { water } \\
\text { added }\end{array}$ & $\begin{array}{c}\text { salt } \\
\text { dissolved }\end{array}$ & $\begin{array}{c}\text { total } \\
\text { supernate }\end{array}$ & $\begin{array}{l}\text { crystallized } \\
\text { salt cake }\end{array}$ & $\begin{array}{c}\text { total } \\
\text { content }\end{array}$ & $\begin{array}{l}\text { Inhib'Water } \\
\text { Disso+Trnsf }\end{array}$ & $\begin{array}{l}210.6 \\
275.0\end{array}$ \\
\hline depth (in) & 256.83 & 40.0 & 16.67 & 58.67 & 240.17 & 298.8 & \multirow[t]{2}{*}{ Contingency } & \multirow[t]{2}{*}{168.0} \\
\hline gallons & 901485 & 140400 & 58500 & 205920 & 842985 & 1048905 & & \\
\hline$\%$ & $85.9 \%$ & $3.4 \%$ & $5.6 \%$ & $19.6 \%$ & $80.4 \%$ & $100.0 \%$ & Sampl\& & 240.0 \\
\hline net hour & & 210.6 & 275.0 & 275.0 & transfer & batch total & (days) & 37.2 \\
\hline
\end{tabular}

\begin{tabular}{|c|c|c|c|c|c|c|c|c|}
\hline Batch \#3 & $\begin{array}{c}\text { starting } \\
\text { level }\end{array}$ & $\begin{array}{l}\text { water } \\
\text { added }\end{array}$ & $\begin{array}{c}\text { salt } \\
\text { dissolved }\end{array}$ & $\begin{array}{c}\text { total } \\
\text { supernate }\end{array}$ & $\begin{array}{c}\text { crystallized } \\
\text { salt cake }\end{array}$ & $\begin{array}{c}\text { total } \\
\text { content }\end{array}$ & $\begin{array}{l}\text { Inhib'Water } \\
\text { Disso+Trnsf }\end{array}$ & $\begin{array}{l}210.6 \\
275.0\end{array}$ \\
\hline depth (in) & 242.17 & 40.0 & 16.67 & 58.67 & 225.50 & 284.2 & \multirow[t]{2}{*}{ Contingency } & \multirow[t]{2}{*}{168.0} \\
\hline gallons & 850005 & 140400 & 58500 & 205920 & 791505 & 997425 & & \\
\hline$\%$ & $85.2 \%$ & $14.1 \%$ & $5.9 \%$ & $20.6 \%$ & $79.4 \%$ & $100.0 \%$ & Sampl\&Prep. & 240.0 \\
\hline net hours & & 210.6 & 275.0 & 275.0 & transfer & batch total & 1 (days) & 37.2 \\
\hline
\end{tabular}




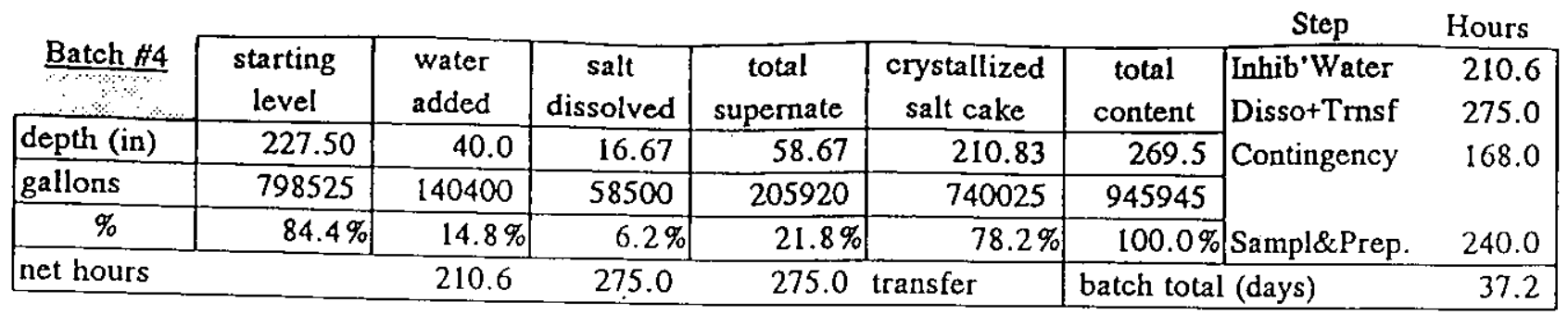

\section{(Case 2) Modified Density Gradient with 25C Inhibited Water}

Tank Level at the End of Batch $4=$

$$
210.8+2=\quad 212.8 * \text { TK Lvl }
$$

Salt Cake Level Reduced $=$

$$
269.5-210.8=
$$

58.7 " TK Lvl

Total Inhibited Water Used=

561600 gallons =

160.0 * TK Lvl

dissolution \& transdfer time (a)

$1100.1 \mathrm{hrs}=$

45.8 days

additional contingency time (b)

$672.0 \mathrm{hrs}$

28.0 days

total dissolution, transf $\&$ contingency $(a+b)=$

73.8 days

inhibited water addition $=$

$842.4 \mathrm{hrs}=$

35.1 days

initial TK decant transfer time $=$

$77.7 \mathrm{hrs}=$

3.2 days

test sample \& preparation $=$ $1200.0 \mathrm{hrs}=$ 50.0 days

total time $=$

3892.2 hrs $=$

162.2 days

or

5.3 . months

After the existing supernate transfer to TK 30 and the above four batch transfer to TK 26, Tank 37 salt level of 212.8 inch will pass the project scope tank level of 227 inches.

Batch $\# 5$ is shown below even though it is not required to meet the original project scope level of 227 inches. The Tank 37 level after Batch 5 will be

\begin{tabular}{|c|c|c|c|c|c|c|c|c|}
\hline & & & & & & & \multicolumn{2}{|l|}{ Step } \\
\hline Batch \#5 & starting & water & salt & total & crystallized & tots & Inhib'Water & 210.6 \\
\hline & level & added & dissolved & supernate & salt cake & content & Disso+Trnsf & 275.0 \\
\hline depth (in) & 212.83 & 40.0 & 16.67 & 58.67 & 196.17 & 254.8 & Contingency & 168.0 \\
\hline gallons & 747045 & 140400 & 58500 & 205920 & 688545 & 894465 & & \\
\hline$\%$ & $83.5 \%$ & $15.7 \%$ & $6.5 \%$ & $23.0 \%$ & $77.0 \%$ & $100.0 \%$ & Sampl\&Prep. & 240.0 \\
\hline net hours & & 210.6 & 275.0 & 275.0 & transfer & batch tota & (days) & 37.2 \\
\hline
\end{tabular}

198.2 inches if Batch 5 is processed. 


\section{TANK 37H DISSOLUTION CYCLE ANALYSIS}

(Case 3) Modified Density Gradient with 50C Inhibited Water

Water to salt dissolution ratio $=$

Modified Density Gradient with 50C IW:

$5.05 \mathrm{gpm}$ dissol. rate; dissol. and contingency $=$

\begin{tabular}{l|l|}
4.87 months total & $100 \%$ \\
\hline
\end{tabular}

a) initial salt cake level (average of $262^{*} \& 277^{\prime \prime}$ ) -

60.2 days

b) inhibited water batch $=\sim 80 \%$ of $10 \mathrm{Kgal}$ tank -.-...--..--

inhibited water pumping to TK 37 (80 to $120 \mathrm{gpm}$ ) -

total time of I batch IW addition to TK $37=$ IWt

IW addition -..-- I batch per $12 \mathrm{hr}$ shift \&

total IW add hr needed/net pumping hr $=I \mathrm{Wt} / \mathrm{IW} \mathrm{p}=12 / 1.33 \ldots$

equiv. continuous IW addition rate $=I W t / I W p=100 \mathrm{gpm} / 9$

batch water add hrs $=(40 \mathrm{in} * 3510 \mathrm{gal} / \mathrm{in}) /(11.11 \mathrm{gpm} * 60 \mathrm{~min} / \mathrm{hr})=$

c) jet transfer TK 37->TK 30 (Existing Decant Before Batch)

d) salt dissolution rate (gpm)

transfer to TK 26 (gpm)

total time (months)

\begin{tabular}{|r|r|r|}
\hline 5.05 & 1.78 & \\
\hline 17.17 & 6.05 & 28.2 \\
\hline 4.87 & 6.81 & \\
\hline
\end{tabular}

832 Avg of $25 \%$ slurry pump rate,

269.50 inch

8000 gallons per batch

$100 \mathrm{gpm} ; \mathrm{IWp}=1.33 \mathrm{hrs}$

$12 \cdot$ hrs

2 batches per day

9

$11.11 \mathrm{gpm}$

210.6 hrs $=8.8$ days

$60 \mathrm{gpm}$ (not Batch Case)

28.29 \& TK10 test with $1.7 \%$ rise $/{ }^{\circ} \mathrm{C}$

$4.45(7.1 * 0.25+8.32) / 2=5.05 \mathrm{gpm}$

Modified Density Gradient with 50C IW:

\begin{tabular}{|c|c|c|c|c|c|c|c|c|}
\hline \multirow{3}{*}{$\begin{array}{l}\text { Decant } \\
\text { Existing }\end{array}$} & \multicolumn{6}{|c|}{ Modified Density Gradient with 50C IW: } & \multicolumn{2}{|l|}{ Step } \\
\hline & salt cake & water & salt & total liq or & crystallized & total & Inhib'Water & 0.0 \\
\hline & + dissolv. & existing & dissolved & supernate & salt cake & content & Dissolution & 0.0 \\
\hline depth (in) & 293.53 & 57.66 & 24.03 & 81.69 & 269.50 & 351.0 & Contingency & 0.0 \\
\hline gallons & 1030278 & 202399 & 84333 & 286732 & 945945 & 1232010 & Jet Transfer & 77.7 \\
\hline$\%$ & $83.6 \%$ & $16.4 \%$ & $6.8 \%$ & $23.3 \%$ & $76.8 \%$ & $100.0 \%$ & Sampl\&Prep. & 240.0 \\
\hline net hours & & 0.0 & 0.0 & 77.7 & transfer & batch total & (days) & 13.2 \\
\hline
\end{tabular}

\begin{tabular}{|c|c|c|c|c|c|c|c|c|}
\hline Batch \#1 & $\begin{array}{c}\text { starting } \\
\text { level }\end{array}$ & $\begin{array}{l}\text { water } \\
\text { added }\end{array}$ & $\begin{array}{c}\text { salt } \\
\text { dissolved } \\
\end{array}$ & $\begin{array}{c}\text { total } \\
\text { supernate }\end{array}$ & $\begin{array}{c}\text { crystallized } \\
\text { salt cake }\end{array}$ & $\begin{array}{c}\text { total } \\
\text { content }\end{array}$ & $\begin{array}{l}\text { Inhib'Water } \\
\text { Disso+Trnsf }\end{array}$ & $\begin{array}{l}210.6 \\
193.1\end{array}$ \\
\hline depth (in) & 271.50 & 40.0 & 16.67 & $58 . \overline{67}$ & 254.83 & 313.5 & \multirow[t]{2}{*}{ Contingency } & \multirow[t]{2}{*}{168.0} \\
\hline gallons & 952965 & 140400 & 58500 & 205920 & 894465 & 1100385 & & \\
\hline$\%$ & $86.6 \%$ & $12.8 \%$ & $5.3 \%$ & $.7 \%$ & $81.3 \%$ & $100.0 \%$ & Sampl & 40.0 \\
\hline et hou & & 210.6 & 193.1 & 193.1 & asfer & batch total & (days) & 33.8 \\
\hline
\end{tabular}

\begin{tabular}{|c|c|c|c|c|c|c|c|c|}
\hline Batch \#2 & $\begin{array}{c}\text { starting } \\
\text { level }\end{array}$ & $\begin{array}{l}\text { water } \\
\text { added }\end{array}$ & $\begin{array}{c}\text { salt } \\
\text { dissolved }\end{array}$ & $\begin{array}{c}\text { total } \\
\text { supernate }\end{array}$ & $\begin{array}{l}\text { crystallized } \\
\text { salt cake }\end{array}$ & $\begin{array}{c}\text { total } \\
\text { content }\end{array}$ & $\begin{array}{l}\text { Inhib'Water } \\
\text { Disso+Tmsf }\end{array}$ & $\begin{array}{l}210.6 \\
193.1\end{array}$ \\
\hline depth (in) & 256.83 & 40.0 & 16.67 & 58.67 & 240.17 & 298.8 & \multirow[t]{2}{*}{ Contingency } & \multirow[t]{2}{*}{168.0} \\
\hline gallons & 901485 & 140400 & 58500 & 205920 & 842985 & 1048905 & & \\
\hline$\%$ & $85.9 \%$ & $13.4 \%$ & $6 \%$ & $19.6 \%$ & $80.4 \%$ & $100.0 \%$ & Sar & 40.0 \\
\hline et hour & & 210.6 & 193.1 & 193.1 & transfer & batch tota & 1 (days) & 33.8 \\
\hline
\end{tabular}

\begin{tabular}{|c|c|c|c|c|c|c|c|c|}
\hline Batch $\# 3$ & $\begin{array}{c}\text { starting } \\
\text { level }\end{array}$ & $\begin{array}{l}\text { water } \\
\text { added }\end{array}$ & $\begin{array}{c}\text { salt } \\
\text { dissolved }\end{array}$ & $\begin{array}{c}\text { total } \\
\text { supernate }\end{array}$ & $\begin{array}{l}\text { crystallized } \\
\text { salt cake }\end{array}$ & $\begin{array}{c}\text { total } \\
\text { content }\end{array}$ & $\begin{array}{l}\text { Inhib'Water } \\
\text { Disso+Tmsf }\end{array}$ & $\begin{array}{l}210.6 \\
193.1\end{array}$ \\
\hline depth (in) & 242.17 & $40 . \overline{0}$ & 16.67 & 58.67 & 225.50 & 284.2 & \multirow[t]{2}{*}{ Contingency } & \multirow[t]{2}{*}{168.0} \\
\hline gallons & 850005 & 140400 & 58500 & 205920 & 791505 & 997425 & & \\
\hline$\%$ & $85.2 \%$ & $14.1 \%$ & $5.9 \%$ & $20.6 \%$ & $79.4 \%$ & $100.0 \%$ & Sampl\&Prep. & 240.0 \\
\hline net hours & & 210.6 & 193.1 & 193.1 & transfer & batch total & (days) & 33.8 \\
\hline
\end{tabular}




\begin{tabular}{|c|c|c|c|c|c|c|c|c|}
\hline & & & & & & & Step & - Hours \\
\hline Batch \#4 & starting & water & & & crystallized & total & Inhib'Water & 210.6 \\
\hline \% & level & added & dissolved & supernate & salt cake & content & Disso+Trnsf & 193.1 \\
\hline depth (in) & 227.50 & 40.0 & 16.67 & 58.67 & 210.83 & 269.5 & Contingency & 168.0 \\
\hline gallons & 798525 & 140400 & 58500 & 205920 & 740025 & $94 \overline{45945}$ & & \\
\hline$\%$ & $84.4 \%$ & $14.8 \%$ & $6.2 \%$ & $21.8 \%$ & $78.2 \%$ & $100.0 \%$ & Sampl\&Prep. & 240.0 \\
\hline net hours & & 210.6 & 193.1 & 193.1 & transfer & batch tota & (days) & 33.8 \\
\hline
\end{tabular}

\section{(Case 3) Modified Density Gradient with 50C Inhibited Water}

Tank Level at the End of Batch $4=$

Salt Cake Level Reduced $=$

Total Inhibited Water Used $=$

dissolution \& transdfer time (a)

additional contingency time (b)

total dissolution, transf \& contingency $(a+b)=$

inhibited water addition $=$

initial TK decant transfer time $=$

test sample \& preparation $=$

total time $=$
$210.8+2=$

$269.5-210.8=$

561600 gallons $=$

$772.3 \mathrm{hrs}=$

$672.0 \mathrm{hrs}$

$842.4 \mathrm{hrs}=$

$77.7 \mathrm{hrs}=$

$1200.0 \mathrm{hrs}=$

$3564.4 \mathrm{hrs}=$
212.8 "TK Lvl

58.7 "TK LvI

$160.0 *$ TK Lvl

32.2 days

28.0 days

60.2 days

35.1 days

3.2 days

50.0 days

or 148.5 days

After the existing supernate transfer to TK 30 and the above four batch transfer to TK 26 ,

Tank 37 salt level of

212.8 inch will pass the project scope tank level of 227 inches.

Batch $\# 5$ is shown below even though it is not required to meet the original project scope level of 227 inches. The Tank 37 level after Batch 5 will be

198.2 inches if Batch 5 is processed.

\begin{tabular}{|c|c|c|c|c|c|c|c|c|}
\hline \multirow{3}{*}{ Batch \#5 } & \multirow{3}{*}{$\begin{array}{c}\text { starting } \\
\text { level }\end{array}$} & \multirow{3}{*}{$\begin{array}{l}\text { water } \\
\text { added }\end{array}$} & \multirow{3}{*}{$\begin{array}{c}\text { salt } \\
\text { dissolved }\end{array}$} & \multirow{3}{*}{$\begin{array}{c}\text { total } \\
\text { supernate }\end{array}$} & \multirow{3}{*}{$\begin{array}{c}\text { crystallized } \\
\text { salt cake }\end{array}$} & \multirow{3}{*}{$\begin{array}{c}\text { total } \\
\text { content }\end{array}$} & Step & \multirow{2}{*}{$\frac{\text { Hours }}{210.6}$} \\
\hline & & & & & & & Inhib'Water & \\
\hline & & & & & & & $\mathrm{Di}$ & 193.1 \\
\hline depth & 21283 & 40.0 & 16.67 & 58.67 & 196.17 & 254.8 & Contingency & 168.0 \\
\hline gallons & $74 \pi$ & 140400 & 58500 & 20 & 688 & 894465 & & \\
\hline$\%$ & $83.5 \%$ & $15.7 \%$ & $\%$ & $0 \%$ & $77.0 \%$ & & Sampl\&Prep. & 240.0 \\
\hline et hour & & 210.6 & 193.1 & 193.1 & transfer & batch total & (days) & 33.8 \\
\hline
\end{tabular}




\section{TANK 37H DISSOLUTION CYCLE ANALYSIS}

\begin{tabular}{|c|c|c|}
\hline $\begin{array}{l}\text { (Case 4) Modified Density Gradient with 75C Inhibited Water } \\
\text { Water to salt dissolution ratio = }\end{array}$ & 2.4 to 1 & $\%$ total time \\
\hline $\begin{array}{l}\text { Modified Density Gradient with 75C IW: } \\
6.56 \mathrm{gpm} \text { dissol. rate; dissol. and contingency }=\end{array}$ & total 52.8 days & $\begin{array}{r}100 \% \\
37 \% \\
\end{array}$ \\
\hline
\end{tabular}

a) initial salt cake level (average of $262^{\prime \prime} \& 277^{\prime \prime}$ ) -

269.50 inch

b) inhibited water batch $=\sim 80 \%$ of $10 \mathrm{Kgal}$ tank inhibited water pumping to TK 37 (80 to $120 \mathrm{gpm}$ ) total time of 1 batch IW addition to TK $37=$ IWt IW addition - 1 batch per $12 \mathrm{hr}$ shift \& total IW add hr needed/net pumping hr $=\mathrm{IWt} / \mathrm{IWp}=12 / 1.33 \ldots$ equiv. continuous IW addition rate $=I W t / I W_{p}=100 \mathrm{gpm} / 9$ batch water add hrs $=(40 \mathrm{in} * 3510 \mathrm{gal} / \mathrm{in}) /(11.11 \mathrm{gpm} * 60 \mathrm{~min} / \mathrm{hr})=$

c) jet transfer TK 37->TK 30 (Existing Decant Before Batch)

$$
\begin{aligned}
8000 & \text { gallons per batch } \\
100 & \text { gpm ; IW } \mathrm{p}=1.33 \mathrm{hrs} \\
12 & \text { hrs } \\
2 & \text { batches per day } \\
9 &
\end{aligned}
$$

$11.11 \mathrm{gpm}$

210.6 hrs $=8.8$ days

\begin{tabular}{|c|c|c|c|c|c|c|c|c|}
\hline \multicolumn{7}{|c|}{ Modified Density Gradient with $75 \mathrm{C}$ IW: } & Step & Hours \\
\hline Decant & salt cake & water & salt & total liq or & crystallized & total & Inhib'Water & 0.0 \\
\hline Exis & + dissolv. & existing & dissolved & supernate & salt cake & content & Dissolution & 0.0 \\
\hline depth (in) & 293.53 & 57.66 & 24.03 & 81.69 & 269.50 & 351.0 & Contingency & 0.0 \\
\hline gallons & 1030278 & 202399 & 84333 & 286732 & 945945 & 1232010 & Jet Transfer & 77.7 \\
\hline$\%$ & $83.6 \%$ & $16.4 \%$ & $6.8 \%$ & $23.3 \%$ & $76.8 \%$ & $100.0 \%$ & Sampl\&Prep. & 240.0 \\
\hline \multicolumn{2}{|l|}{ net hours } & 0.0 & 0.0 & 77.7 & transfer & batch total & (days) & 13.2 \\
\hline
\end{tabular}

60 gpm (not Batch Case)

\begin{tabular}{|c|c|c|c|c|c|c|c|c|}
\hline Batch \#1 & $\begin{array}{c}\text { starting } \\
\text { level }\end{array}$ & $\begin{array}{l}\text { water } \\
\text { added }\end{array}$ & $\begin{array}{c}\text { salt } \\
\text { dissolved }\end{array}$ & $\begin{array}{c}\text { total } \\
\text { supernate }\end{array}$ & $\begin{array}{c}\text { crystallized } \\
\text { salt cake }\end{array}$ & $\begin{array}{c}\text { total } \\
\text { content }\end{array}$ & $\begin{array}{l}\text { Inhib'Water } \\
\text { Disso+Trusf }\end{array}$ & $\begin{array}{l}210.6 \\
148.5\end{array}$ \\
\hline depth (in) & 271.50 & 40.0 & 16.67 & 58.67 & 254.83 & 313.5 & \multirow[t]{2}{*}{ Contingency } & \multirow[t]{2}{*}{168.0} \\
\hline gallons & 952965 & 140400 & 58500 & 205920 & 894465 & 1100385 & & \\
\hline$\%$ & $86.6 \%$ & $12.8 \%$ & $5.3 \%$ & $18.7 \%$ & $81.3 \%$ & $100.0 \%$ & Sampl & 240.0 \\
\hline \multicolumn{2}{|l|}{ net hours } & 210.6 & 148.5 & 148.5 & transfer & batch total & (days) & 32.0 \\
\hline
\end{tabular}

d) salt dissolution rate ( $\mathrm{gpm})$

\begin{tabular}{|c|c|c|c|c|c|c|c|c|}
\hline Batch 122 & $\begin{array}{c}\text { starting } \\
\text { level }\end{array}$ & $\begin{array}{l}\text { water } \\
\text { added }\end{array}$ & $\begin{array}{c}\text { salt } \\
\text { dissolved }\end{array}$ & $\begin{array}{c}\text { total } \\
\text { supernate }\end{array}$ & $\begin{array}{l}\text { crystallized } \\
\text { salt cake }\end{array}$ & $\begin{array}{c}\text { total } \\
\text { content }\end{array}$ & $\begin{array}{l}\text { Inbib'Water } \\
\text { Disso+Trusf }\end{array}$ & $\begin{array}{l}210.6 \\
148.5\end{array}$ \\
\hline depth (in) & 256.83 & 40.0 & 16.67 & 58.67 & 240.17 & 298.8 & \multirow[t]{2}{*}{ Contingency } & 168.0 \\
\hline gallons & 901485 & 140400 & 58500 & 205920 & 842985 & 1048905 & & \\
\hline$\%$ & $85.9 \%$ & $13.4 \%$ & $5.6 \%$ & $19.6 \%$ & $80.4 \%$ & $100.0 \%$ & Sampl\&Prep. & 240.0 \\
\hline \multicolumn{2}{|l|}{ net hours } & 210.6 & 148.5 & 148.5 & transfer & batch total & (days) & 32.0 \\
\hline
\end{tabular}

\begin{tabular}{|l|r|r|r|r|}
\hline d) salt dissolution rate $(\mathrm{gpm})$ & 6.56 & 2.33 & 10.80 & Avg of $25 \%$ slurry pump rate, \\
\cline { 1 - 4 } transfer to TK 26 (gpm) & 22.32 & 7.91 & 36.73 & \& TK10 test with $1.7 \%$ rise $/{ }^{\circ} \mathrm{C}$ \\
total time (months) & 4.63 & 6.11 & 4.31 & $(9.3 / 4+10.804) / 2=6.56 \mathrm{gpm}$ \\
\hline
\end{tabular}

\begin{tabular}{|c|c|c|c|c|c|c|c|c|}
\hline Batch $\# 3$ & $\begin{array}{c}\text { starting } \\
\text { level }\end{array}$ & $\begin{array}{l}\text { water } \\
\text { added }\end{array}$ & $\begin{array}{c}\text { salt } \\
\text { dissolved }\end{array}$ & $\begin{array}{c}\text { total } \\
\text { supernate }\end{array}$ & $\begin{array}{c}\text { crystallized } \\
\text { salt cake }\end{array}$ & $\begin{array}{c}\text { total } \\
\text { content }\end{array}$ & $\begin{array}{l}\text { Inhib'Water } \\
\text { Disso+Trnsf }\end{array}$ & $\begin{array}{l}210.6 \\
148.5\end{array}$ \\
\hline depth (in) & 242.17 & 40.0 & 16.67 & 58.67 & 225.50 & 284.2 & \multirow[t]{2}{*}{ Contingency } & \multirow[t]{2}{*}{168.0} \\
\hline gallons & 850005 & 140400 & 58500 & 205920 & 791505 & 997425 & & \\
\hline$\%$ & $85.2 \%$ & $14.1 \%$ & $5.9 \%$ & $20.6 \%$ & $79.4 \%$ & $100.0 \%$ & Sampl\&Prep. & 240.0 \\
\hline \multicolumn{2}{|l|}{ net hours } & 210.6 & 148.5 & 148.5 & transfer & batch total & 1 (days) & 32.0 \\
\hline
\end{tabular}

Modified Density Gradient with 75C IW: 
WSRC-TR-2001-00277, Rev. 0

\begin{tabular}{|c|c|c|c|c|c|c|c|c|}
\hline \multirow{3}{*}{ Batch \#4 } & & & & & & & Step & Hours \\
\hline & starting & water & & & crystallizéd & total & Inhib'Water & 210.6 \\
\hline & level & added & dissolved & supernate & salt cake & content & Disso+Trnsf & 148.5 \\
\hline depth (in) & 227.50 & 40.0 & $\cdot \overline{16.67}$ & 58.67 & 210.83 & 269.5 & Contingency & 168.0 \\
\hline gallons & 798525 & 140400 & 58500 & 205920 & 740025 & 945945 & & \\
\hline$\%$ & $84.4 \%$ & $14.8 \%$ & $6.2 \%$ & $21.8 \%$ & $78.2 \%$ & $100.0 \%$ & Sampl\&Prep. & 240.0 \\
\hline net hours & & 210.6 & 148.5 & 148.5 & transfer & batch tota & (days) & 32.0 \\
\hline
\end{tabular}

\section{(Case 4) Modified Density Gradient with 75C Inhibited Water}

Tank Level at the End of Batch $4=$

Salt Cake Level Reduced $=$

Total Inhibited Water Used $=$

dissolution \& transdfer time (a)

additional contingency time (b)

total dissolution, transf \& contingency $(a+b)=$

inhibited water addition $=$

initial $\mathrm{TK}$ decant transfer time $=$

test sample \& preparation=

total time $=$

$$
210.8+2=
$$

$269.5-210.8=$

56.1600 gallons $=$

594.1 hrs $=$

$672.0 \mathrm{hrs}$

$842.4 \mathrm{hrs}=$

77.7 hrs $=$

$1200.0 \mathrm{hrs}=$

3386.2 hrs $=$
212.8 " TK Lvl

$58.7 *$ TK LvI

160.0 "TK Lvl

24.8 days

28.0 days

52.8 days

35.1 days

3.2 days

50.0 days

141.1 days

After the existing supernate transfer to TK 30 and the above four batch transfer to TK 26,

Tank 37 salt level of

212.8 inch will pass the project scope tank level of 227 inches.

Batch \#5 is shown below even though it is not required to meet the original project scope level of 227 inches. The Tank 37 level after Batch 5 will be

198.2 inches if Batch 5 is processed.

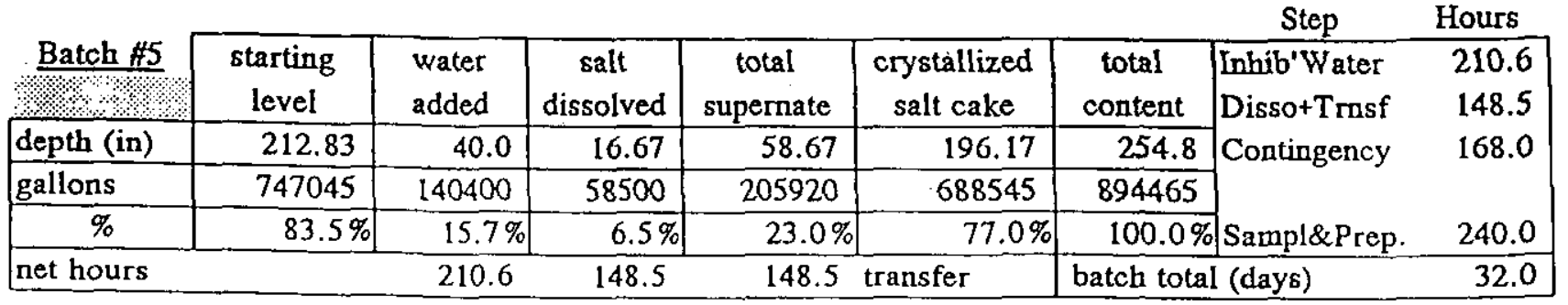




\section{TANK 37H DISSOLUTION CYCLE ANALYSIS}

(Case 5) One Flygt Mixer with 25C Inhibited Water

Water to salt dissolution ratio $=$

One Flygt Mixer with 25C IW:

$3.00 \mathrm{gpm}$ dissol. rate; dissol. and contingency $=$

5.89 months total

2.4 to 1

\begin{tabular}{|r|}
\hline$\%$ total time \\
\hline $100 \%$ \\
$46 \%$ \\
\hline
\end{tabular}

a) initial salt cake level (average of $262^{\prime \prime} \& 277^{\prime \prime}$ ) -

82.2 days

b) inhibited water batch $=\sim 80 \%$ of $10 \mathrm{Kgal}$ tank -

inhibited water pumping to TK 37 ( 80 to $120 \mathrm{gpm}$ )

total time of 1 batch IW addition to TK $37=$ IWt

IW addition ----.--..-- 1 batch per $12 \mathrm{hr}$ shift \&

total IW add hr needed/net pumping $\mathrm{hr}=\mathrm{IW} / \mathrm{IWp}=12 / 1.33$

equiv. continuous IW addition rate $=I W / / W_{p}=100 \mathrm{gpm} / 9$----

batch water add hrs $=(40 \mathrm{in} * 3510 \mathrm{gal} / \mathrm{in}) /(11.11 \mathrm{gpm} * 60 \mathrm{~min} / \mathrm{hr})=$

c) jet transfer TK $37->$ TK 30 and or TK 26

\begin{tabular}{|c|r|r|r|}
\hline d) salt dissolution rate (gpm) & used here & low & high \\
\hline total time (months) & 5.00 & 2.00 & 4.00 \\
\hline
\end{tabular}

269.50 inch

8000 gallons per batch

$100 \mathrm{gpm} ; \mathrm{rWp}=1.33 \mathrm{hrs}$

12 hrs

2 batches per day

9

$11.11 \mathrm{gpm}$

210.6 hrs $=8.8$ days

$60 \mathrm{gpm}$

One Flygt Mixer with 25C IW:

\begin{tabular}{|c|c|c|c|c|c|c|c|c|}
\hline & & & & & & & Step & Hours \\
\hline Existing & salt cake & water & salt & total liq or & crystallized & total & Inhib'Water & 0.0 \\
\hline depth (in) & t aissolv. & existing & dissolved & supernate & salt cake & content & Dissolution & 0.0 \\
\hline \begin{tabular}{|l} 
gallons \\
\end{tabular} & 293.53 & 57.66 & 24.03 & 81.69 & 269.50 & 351.0 & Contingency & 0.0 \\
\hline gallons & 1030278 & 202399 & 84333 & 286732 & 945945 & 1232010 & Jet Transfer & 77.7 \\
\hline \multirow{2}{*}{\multicolumn{2}{|c|}{ net hours }} & $16.4 \%$ & $6.8 \%$ & $23.3 \%$ & $76.8 \%$ & $100.0 \%$ & Sampl\&Prep. & 240.0 \\
\hline & & 0.0 & 0.0 & 77.7 & transfer & batch total & (days) & 13.2 \\
\hline
\end{tabular}

\begin{tabular}{|c|c|c|c|c|c|c|c|c|}
\hline Batch \#1 & $\begin{array}{c}\text { starting } \\
\text { Ievel }\end{array}$ & $\begin{array}{l}\text { water } \\
\text { added }\end{array}$ & $\begin{array}{c}\text { salt } \\
\text { dissolved } \\
\end{array}$ & $\begin{array}{c}\text { total } \\
\text { supernate }\end{array}$ & $\begin{array}{l}\text { crystallized } \\
\text { salt cake }\end{array}$ & $\begin{array}{c}\text { total } \\
\text { content }\end{array}$ & $\begin{array}{l}\text { Inhib'Water } \\
\text { Dissolution }\end{array}$ & $\begin{array}{l}210.6 \\
325.0\end{array}$ \\
\hline depth (in) & 271.50 & 40.0 & 16.67 & 58.67 & 254.83 & 313.5 & Contingency & 168.0 \\
\hline gallons & 952965 & 140400 & 58500 & 205920 & 894465 & 1100385 & Jet Trar & 55.3 \\
\hline$\%$ & $86.6 \%$ & $12.8 \%$ & $5.3 \%$ & $18.7 \%$ & $81.3 \%$ & $100.0 \%$ & SampI\&Prep. & 240.0 \\
\hline net hours & & 210.6 & 325.0 & 55.3 & transfer & batch total & 1 (days) & 41.6 \\
\hline
\end{tabular}

\begin{tabular}{|c|c|c|c|c|c|c|c|c|}
\hline Batch \#2 & $\begin{array}{c}\text { starting } \\
\text { level }\end{array}$ & $\begin{array}{l}\text { water } \\
\text { added }\end{array}$ & $\begin{array}{c}\text { salt } \\
\text { dissolved }\end{array}$ & $\begin{array}{c}\text { total } \\
\text { supernate }\end{array}$ & $\begin{array}{c}\text { crystallized } \\
\text { salt cake }\end{array}$ & $\begin{array}{c}\text { total } \\
\text { content }\end{array}$ & $\begin{array}{l}\text { Inhib'Water } \\
\text { Dissolution }\end{array}$ & $\begin{array}{l}210.6 \\
325.0\end{array}$ \\
\hline depth (in) & 256.83 & 40.0 & .16 .67 & 58.67 & 240.17 & 298.8 & Contingency & 168.0 \\
\hline gallons & 901485 & 140400 & 58500 & 205920 & 842985 & 1048905 & Jet Transfer & 55.3 \\
\hline$\%$ & $85.9 \%$ & $13.4 \%$ & $5.6 \%$ & $19.6 \%$ & $80.4 \%$ & $100.0 \%$ & Sampl\&Prep. & 240.0 \\
\hline \multicolumn{2}{|l|}{ net hours } & 210.6 & 325.0 & 55.3 & transfer & batch total & (days) & 41.6 \\
\hline
\end{tabular}

\begin{tabular}{|c|c|c|c|c|c|c|c|c|}
\hline Batch \#3 & $\begin{array}{c}\text { starting } \\
\text { level }\end{array}$ & $\begin{array}{r}\text { water } \\
\text { added }\end{array}$ & $\begin{array}{c}\text { salt } \\
\text { dissolved }\end{array}$ & $\begin{array}{c}\text { total } \\
\text { supernate }\end{array}$ & $\begin{array}{c}\text { crystallized } \\
\text { salt cake }\end{array}$ & $\begin{array}{c}\text { total } \\
\text { content }\end{array}$ & $\begin{array}{l}\text { Inhib:Water } \\
\text { Dissolution }\end{array}$ & $\begin{array}{l}210.6 \\
325.0\end{array}$ \\
\hline depth (in) & 242.17 & 40.0 & $16 . \overline{67}$ & 58.67 & 225.50 & 284.2 & Contingency & 168.0 \\
\hline gallons & 850005 & 140400 & 58500 & 205920 & 791505 & 997425 & Jet Transfer & 55.3 \\
\hline$\%$ & $85.2 \%$ & $14.1 \%$ & $5.9 \%$ & $20.6 \%$ & $79.4 \%$ & $100.0 \%$ & SampI\&Prep. & 240.0 \\
\hline net hours & & 210.6 & 325.0 & 55.3 & transfer & batch total & (days) & 41.6 \\
\hline
\end{tabular}




\begin{tabular}{|c|c|c|c|c|c|c|c|c|}
\hline \multicolumn{9}{|r|}{ Hours } \\
\hline Batch \#4 & starting & water & & & crystallized & total & Inhib'Water & 210.6 \\
\hline & level & added & dissolved & supernate & salt cake & content & Dissolution & 325.0 \\
\hline depth (in) & 227.50 & 40.0 & 16.67 & 58.67 & 210.83 & 269.5 & Contingency & 168.0 \\
\hline gallons & 798525 & 140400 & 58500 & 205920 & 740025 & 945945 & Jet Transfer & 55.3 \\
\hline$\%$ & $84.4 \%$ & $14.8 \%$ & $6.2 \%$ & $21.8 \%$ & $78.2 \%$ & $100.0 \%$ & Sampl\&Prep. & 240.0 \\
\hline \multicolumn{2}{|l|}{ net hours } & 210.6 & 325.0 & 55.3 & transfer & batch tota & (days) & 41.6 \\
\hline
\end{tabular}

\section{(Case 5) One Flygt Mixer with 25C Inhibited Water}

Tank Level at the End of Batch $4=$

Salt Cake Level Reduced $=$

Total Inhibited Water Used=

dissolution \& transdfer time (a)

additional contingency time (b)

total dissolution, transf \& contingency $(a+b)=$

inhibited water addition $=$

initial TK decant transfer time $=$

test sample $\&$ preparation=

total time $=$
$210.8+2=$

$269.5-210.8=$

561600 gallons $=$

$1300.0 \mathrm{hrs}=$

$672.0 \mathrm{hrs}$

$842.4 \mathrm{hrs}=$

$298.7 \mathrm{hrs}=$

$1200.0 \mathrm{hrs}=$

$4313.1 \mathrm{hrs}=$
212.8 "TK Lvl

$58.7 " \mathrm{TK} \mathrm{LvI}$

160.0 " TK Lvl

54.2 days

28.0 days

82.2 days

35.1 days

12.4 days

50.0 days

179.7 days

or 5.9 months

After the existing supernate transfer to TK 30 and the above four batch transfer to TK 26, Tank 37 salt level of 212.8 inch will pass the project scope tank level of 227 inches.

Batch $\$ 5$ is shown below even though it is not required to meet the original project scope level of 227 inches. The Tank 37 level after Batch 5 will be

198.2 inches if Batch 5 is processed.

\begin{tabular}{|c|c|c|c|c|c|c|c|c|}
\hline \multirow{3}{*}{ Batch \#5 } & \multirow{3}{*}{$\begin{array}{c}\text { starting } \\
\text { level }\end{array}$} & \multirow{3}{*}{$\begin{array}{l}\text { water } \\
\text { added }\end{array}$} & \multirow{3}{*}{\begin{tabular}{|c|} 
salt \\
dissolved
\end{tabular}} & \multirow{3}{*}{$\begin{array}{c}\text { total } \\
\text { supernate }\end{array}$} & \multirow{3}{*}{$\begin{array}{c}\text { crystallized } \\
\text { salt cake }\end{array}$} & \multirow{3}{*}{$\begin{array}{c}\text { total } \\
\text { content }\end{array}$} & Step & \multirow{2}{*}{$\frac{\text { Hours }}{210.6}$} \\
\hline & & & & & & & Inhib'Water & \\
\hline & & & & & & & Dissolution & 325.0 \\
\hline depth (in) & 212.83 & 40.0 & 16.67 & 58.67 & 196.17 & 254.8 & Contingency & 168.0 \\
\hline gallons & 747045 & 140400 & 58500 & 205920 & 688545 & 894465 & Jet Transfer & 55.3 \\
\hline$\%$ & $83.5 \%$ & $15.7 \%$ & $6.5 \%$ & $23.0 \%$ & $77.0 \%$ & $100.0 \%$ & Sampl\&Prep. & 240.0 \\
\hline net hour & & 210.6 & 325.0 & 55.31 & transfer & batch tota & 1 (days) & 41.6 \\
\hline
\end{tabular}


TANK 37H DISSOLUTION CYCLE ANALYSIS

\begin{tabular}{|c|c|c|c|}
\hline (Case 6) Two Flygt Mixers with 25C Amb & & & \\
\hline Water to salt dissolution ratio $=$ & 2.4 to & 1 & $\%$ total time \\
\hline $\begin{array}{lc}2 \text { Flygt Mixers with 25C Ambient IW: } & 5.4 \mathrm{~m} \\
4.2 \mathrm{gpm} \text { dissolution rate: dissolution \& mixing }=\end{array}$ & total & & $00 \%$ \\
\hline
\end{tabular}

a) initial salt cake level (average of $262^{\prime \prime} \& 277^{*}$ ) =

b) inhibited water batch $=\sim 80 \%$ of $10 \mathrm{Kgal}$ tank $=$ inhibited water pumping to TK 37 (80 to $120 \mathrm{gpm})=$ net pumping time of one batch IW to TK $37=$ IWp $=$ number of IW batches per $12 \mathrm{hr}$-shift $=$ number of IW batches per day = total time of 1 batch IW addition to TK $37=$ IWt $=$ total IW time needed/net pumping time $=$ IW $/ / \mathrm{W} p=$ 269.50 inch 8000 gallons $100 \mathrm{gpm}$

1.3 hrs

1 batch per shift

2 batches per day

12 brs

9

c) salt dissolution rate $=$

$4.2 \mathrm{gpm}$

d) jet transfer rate from TK 37 to TK 30 or TK $26=$ (60 to $100 \mathrm{gpm}$ )

$60 \mathrm{gpm}$

2 Flygt Mixers with 25C Ambient IW:

Step Hours

\begin{tabular}{|c|c|c|c|c|c|c|c|c|}
\hline \multirow{2}{*}{ Decant } & & \multirow{2}{*}{\multicolumn{2}{|c|}{$\frac{\text { step }}{\text { Inhib'y/atar }}$}} \\
\hline & salt cake & water & salt & total liq or & crystallized & total & & \\
\hline Exisung & + dissolv. & existing & dissolved & supernate & sal & content & Dissolution & 0.0 \\
\hline depth (in) & 293.53 & 57.66 & 24.03 & 81.69 & 269.50 & 351.0 & ExtraMixing & 0.0 \\
\hline gallons & 1030278 & 202399 & 84333 & 286732 & 945945 & 1232 & Jet Transfer & 77.7 \\
\hline$\%$ & $83.6 \%$ & $16.4 \%$ & $6.8 \%$ & $23.3 \%$ & $76.8 \%$ & $100.0 \%$ & Sampl\&Prep. & 240.0 \\
\hline et hours & & 0.0 & 0.0 & 77.7 & transfer & batch total & (days) & 13.2 \\
\hline
\end{tabular}

\begin{tabular}{|c|c|c|c|c|c|c|c|c|}
\hline Batch \#1 & $\begin{array}{c}\text { starting } \\
\text { level }\end{array}$ & $\begin{array}{l}\text { water } \\
\text { added }\end{array}$ & $\begin{array}{c}\text { salt } \\
\text { sicslof }\end{array}$ & total & crystallized & total & Inhib'Water & $\begin{array}{l}210.6 \\
232.1\end{array}$ \\
\hline depth (in) & 271.50 & 40.0 & 16.67 & 58.67 & 254.83 & 313.5 & ExtraMixing & 168.0 \\
\hline gallons & 952965 & 140400 & 58500 & 205920 & 894465 & 1100385 & Jet Transfer & 55.3 \\
\hline$\%$ & $86.6 \%$ & $12.8 \%$ & $5.3 \%$ & $18.7 \%$ & $81.3 \%$ & $100.0 \%$ & Sampl\&Prep. & 240.0 \\
\hline net hours & & 210.6 & 232.1 & 55.3 & transfer & batch total & (days) & 37.7 \\
\hline
\end{tabular}

\begin{tabular}{|c|c|c|c|c|c|c|c|c|}
\hline Batch \#2 & $\begin{array}{c}\text { starting } \\
\text { level }\end{array}$ & $\begin{array}{l}\text { water } \\
\text { added }\end{array}$ & $\begin{array}{c}\text { salt } \\
\text { dissolved }\end{array}$ & $\begin{array}{c}\text { total } \\
\text { supernate }\end{array}$ & $\begin{array}{l}\text { crystallized } \\
\text { salt cake }\end{array}$ & $\begin{array}{c}\text { total } \\
\text { content }\end{array}$ & $\begin{array}{l}\text { Inhib'Water } \\
\text { Dissolution }\end{array}$ & $\begin{array}{l}210.6 \\
232.1\end{array}$ \\
\hline depth (in) & 256.83 & 40.0 & 16.67 & 58.67 & 240.17 & 298.8 & Extra & 168.0 \\
\hline gallons & 01485 & 40400 & 58500 & 205920 & 842985 & 1048905 & Jet $\mathrm{Tr}$ & 55.3 \\
\hline$\%$ & $85.9 \%$ & $13.4 \%$ & $5.6 \%$ & $19.6 \%$ & $80.4 \%$ & $100.0 \%$ & Sampl\& & 240.0 \\
\hline net hours & & 210.6 & 232.1 & $\overline{55.3}$ & transfer & batch total & 1 (days) & 37.7 \\
\hline
\end{tabular}

\begin{tabular}{|c|c|c|c|c|c|c|c|c|}
\hline Batch \#3 & $\begin{array}{c}\text { starting } \\
\text { level }\end{array}$ & $\begin{array}{l}\text { water } \\
\text { added }\end{array}$ & $\begin{array}{c}\text { salt } \\
\text { dissolved }\end{array}$ & $\begin{array}{c}\text { total } \\
\text { supernate }\end{array}$ & $\begin{array}{c}\text { crystallized } \\
\text { salt cake }\end{array}$ & $\begin{array}{c}\text { total } \\
\text { content }\end{array}$ & $\begin{array}{l}\text { Inhib'Water } \\
\text { Dissolution }\end{array}$ & $\begin{array}{l}210.6 \\
232.1\end{array}$ \\
\hline depth (in) & 242.17 & 40.0 & 16.67 & 58.67 & 225.50 & 284.2 & ExtraMixing & 168.0 \\
\hline gallons & 850005 & 140400 & 58500 & 205920 & 791505 & 997425 & Jet Transfer & 55.3 \\
\hline \% & $85.2 \%$ & $14.1 \%$ & $5.9 \%$ & $20.6 \%$ & $79.4 \%$ & $100.0 \%$ & Sampl\&Prep. & 240.0 \\
\hline thours & & 210.6 & 232.1 & 55.3 & transfer & batch total & (days) & 37.7 \\
\hline
\end{tabular}




\begin{tabular}{|c|c|c|c|c|c|c|c|c|}
\hline & & & & & & & Step & Hours \\
\hline Batch $f t$ & starting & water & & total & crystallized & & Inhib'Water & 210.6 \\
\hline & level & added & dissolved & supernate & salt & content & Dissol & 232.1 \\
\hline depth (in) & 227.50 & 40.0 & 16.67 & 58.67 & 210.83 & 269.5 & ing & 168.0 \\
\hline gallons & 798525 & 40400 & 58500 & 205920 & 740025 & 945945 & Jet Tra & 55.3 \\
\hline$\%$ & $84.4 \%$ & $14.8 \%$ & $6.2 \%$ & $21.8 \%$ & $78.2 \%$ & $100.0 \%$ & Sampl\&Prep. & 240.0 \\
\hline \multicolumn{2}{|l|}{ net hours } & 210.6 & 232.1 & 55.3 & transfer & batch tota & ys) & 37.7 \\
\hline
\end{tabular}

\section{(Case 6) Two Flygt Mixers with 25C Ambient Inhibited Water}

Tank Level at the End of Batch $4=$

Salt Cake Level Reduced $=$

Total Inhibited Water Used=

dissolution with mixer (a) =

extra mixing (b)

total mixer running time $(a+b)=$

inhibited water addition $=$

jet transfer time $=$

test sample \& preparation $=$

total time $=$
$210.8+2=$

$269.5-210.8=$

561600 gallons $=$

$928.6 \mathrm{hrs}=$

$672.0 \mathrm{hrs}$

842.4 hrs $=$

$298.7 \mathrm{hrs}=$

$1200.0 \mathrm{hrs}=$

3941.7 hrs $=$
212.8 "TK Lvl

$58.7 *$ TK Lvl

160.0 " TK Lvl

38.7 days

28.0 days

66.7 days

35.1 days

12.4 days

50.0 days

164.2 days

5.4 months

After the existing supernate transfer to TK 30 and the above four batch transfer to TK 26,

Tank 37 salt level of 212.8 inch will pass the project scope tank level of 227 inches.

Batch $\$ 5$ is shown below even though it is not required to meet the original project scope level of 227 inches. The Tank 37 level after Batch 5 will be

198.2 inches if Batch 5 is processed.

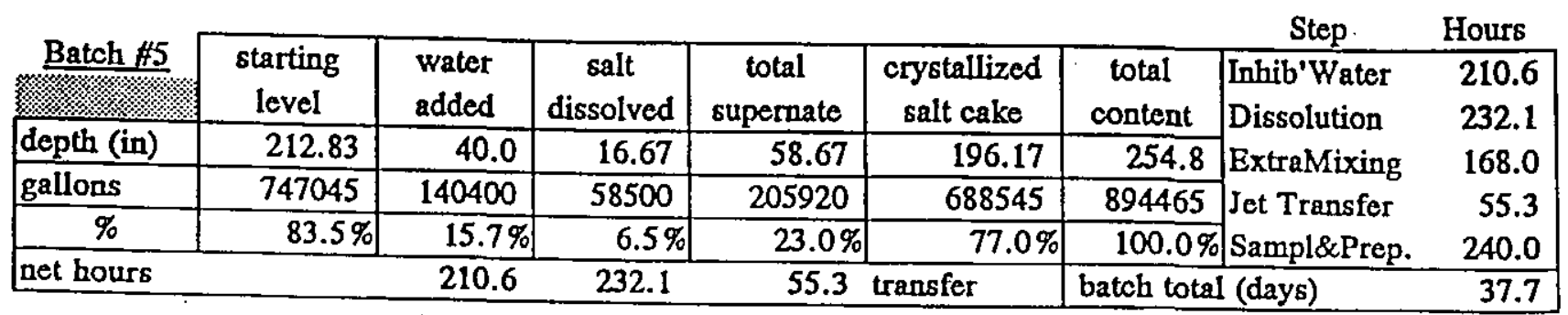




\section{TANK 37H DISSOLUTION CYCLE ANALYSIS}

\begin{tabular}{|c|c|c|c|c|}
\hline Case & & & & \\
\hline Water to salt dissolution ratio $=$ & 2.4 & to & 1 & $\%$ total time \\
\hline $\begin{array}{l}\text { 1 Flygt Mixer with 50C Heated IW: } \\
4.3 \mathrm{gpm} \text { dissolution rate; dissolution \& mixing }=\end{array}$ & $\begin{array}{l}\text { total } \\
65.8 \\
\end{array}$ & days & & $\begin{array}{r}100 \% \\
40 \%\end{array}$ \\
\hline
\end{tabular}

a) initial salt cake level (average of $262^{*} \& 277^{\prime \prime}$ ) =

269.50 inch

b) inhibited water batch $=\sim 80 \%$ of $10 \mathrm{Kgal}$ tank $=$ inhibited water pumping to TK $37(80$ to $120 \mathrm{gpm})=$ 8000 gallons net pumping time of one batch IW to TK $37=$ IWp $=$ $100 \mathrm{gpm}$ number of IW batches per $12 \mathrm{br}$-shift $=$

$1.3 \mathrm{hrs}$ number of IW batches per day = total time of 1 batch IW addition to TK $37=$ IWt $=$ total IW time needed/net pumping time $=$ IW UIWp $=$

1 batch per shift

2 batches per day

12 hrs

c) salt dissolution rate $=$

9

d) jet transfer rate from TK 37 to TK 30 or TK $26=$ (60 to $100 \mathrm{gpm}$ )

\section{$4.3 \mathrm{gpm}$ \\ $60 \mathrm{gpm}$}

1 Flygt Mixer with 50C Heated IW:

\begin{tabular}{|c|c|c|c|c|c|c|c|c|}
\hline & & I Flygt Mi & xer with 50 & C Heated IW & & & Step & Hours \\
\hline Decant & salt cake & water & salt & total liq or & crystallized & tolat & Inhib'Water & 0.0 \\
\hline Existing & + dissolv. & existing & dissolved & supernate & salt cake & content & Dissolution & 0.0 \\
\hline depth (in) & 293.53 & 57.66 & 24.03 & 81.69 & 269.50 & 351.0 & ExtraMixing & 0.0 \\
\hline gallons & 1030278 & 202399 & 84333 & 286732 & 945945 & 1232010 & Jet Transfer & 77.7 \\
\hline$\%$ & $83.6 \%$ & $16.4 \%$ & $6.8 \%$ & $23.3 \%$ & $76.8 \%$ & $100.0 \%$ & Sampl\&Prep. & 240.0 \\
\hline net hours & & 0.0 & 0.0 & 77.7 & transfer & batch tota & (days) & 13.2 \\
\hline
\end{tabular}

\begin{tabular}{|c|c|c|c|c|c|c|c|c|}
\hline Batch \#1 & starting & water & salt & total & crystallized & total & Inhib'Water & 210.6 \\
\hline depth (in) & 27150 & & aissolved & & & Content & $\mathrm{Dis}$ & 6.7 \\
\hline gallons & 5006 & 40.0 & 16.67 & 58.67 & 254.83 & 313.5 & ExtraMixing & 168.0 \\
\hline$\%$ & 932903 & 40400 & 58500 & 2059.20 & 894465 & 1100385 & Jet Transfer & 55.3 \\
\hline \multirow{2}{*}{\multicolumn{2}{|c|}{ net hours }} & $12.8 \%$ & $5.3 \%$ & $18.7 \%$ & $81.3 \%$ & $100.0 \%$ & Sampl\&Prep. & 240.0 \\
\hline & & 210.6 & 226.7 & 55.3 & transfer & batch total & (days) & 37.5 \\
\hline
\end{tabular}

\begin{tabular}{|c|c|c|c|c|c|c|c|c|}
\hline Batch \#2 & $\begin{array}{c}\text { starting } \\
\text { level }\end{array}$ & $\begin{array}{l}\text { water } \\
\text { added }\end{array}$ & $\begin{array}{c}\text { salt } \\
\text { dissolved }\end{array}$ & $\begin{array}{c}\text { total } \\
\text { supernate }\end{array}$ & $\begin{array}{l}\text { crystallized } \\
\text { salt cake }\end{array}$ & $\begin{array}{c}\text { total } \\
\text { content. }\end{array}$ & $\begin{array}{l}\text { Inhib'Water } \\
\text { Dissolution }\end{array}$ & $\begin{array}{l}210.6 \\
226.7\end{array}$ \\
\hline depth (in) & 256.83 & 40.0 & 16.67 & 58.67 & 240.17 & 298.8 & ExtraMixing & 168.0 \\
\hline gallons & 901485 & 140400 & 58500 & 205920 & 842985 & 1048905 & Jet Transfer & 55.3 \\
\hline$\%$ & $85.9 \%$ & $13.4 \%$ & $5.6 \%$ & $19.6 \%$ & $80.4 \%$ & $100.0 \%$ & Sampl\&Prep. & 240.0 \\
\hline net hours & & 210.6 & 226.7 & 55.3 & transfer & batch tota & 1 (days) & 37.5 \\
\hline
\end{tabular}

\begin{tabular}{|c|c|c|c|c|c|c|c|c|}
\hline Batch \#3 & $\begin{array}{c}\text { starting } \\
\text { level }\end{array}$ & $\begin{array}{l}\text { water } \\
\text { added }\end{array}$ & $\begin{array}{c}\text { salt } \\
\text { dissolved }\end{array}$ & $\begin{array}{c}\text { total } \\
\text { supernate }\end{array}$ & $\begin{array}{c}\text { crystallized } \\
\text { salt cake }\end{array}$ & $\begin{array}{c}\text { total } \\
\text { content }\end{array}$ & $\begin{array}{l}\text { Inhib'Water } \\
\text { Dissolution }\end{array}$ & $\begin{array}{l}210.6 \\
226.7\end{array}$ \\
\hline depth (in) & 242.17 & 40.0 & 16.67 & 58.67 & 225.50 & 284.2 & ExtraMixing & 168.0 \\
\hline gallons & 850005 & 140400 & 58500 & 205920 & 791505 & 997425 & Jet Transfer & 55.3 \\
\hline$\%$ & $85.2 \%$ & $14.1 \%$ & $5.9 \%$ & $20.6 \%$ & $79.4 \%$ & $100.0 \%$ & Sampl\&Prep. & 240.0 \\
\hline net hours & & 210.6 & 226.7 & 55.3 & transfer & batch tota & I (days) & 37.5 \\
\hline
\end{tabular}




\begin{tabular}{|c|c|c|c|c|c|c|c|c|}
\hline \multicolumn{9}{|r|}{ Hours } \\
\hline Batch \#4 & starting & water & salt & total & crystallized & tots & Inhib'Water & 210.6 \\
\hline & level & added & dissolved & supernate & salt cake & content & Dissolution & 226.7 \\
\hline depth (in) & 227.50 & 40.0 & 16.67 & 58.67 & 210.83 & 269.5 & ExtraMixing & 168.0 \\
\hline gallons & 798525 & 140400 & 58500 & 205920 & 740025 & 945945 & Jet Transfer & 55.3 \\
\hline$\%$ & $84.4 \%$ & $14.8 \%$ & $6.2 \%$ & $21.8 \%$ & $78.2 \%$ & $100.0 \%$ & Sampl\&Prep. & 240.0 \\
\hline net bours & & 210.6 & 226.7 & 55.3 & transfer & batch total & (days) & 37.5 \\
\hline
\end{tabular}

\section{(Case 7) One Flygt Mixer with 50C Heated Inhibited Water}

Tank Level at the End of Batch $4=$

Salt Cake Level Reduced $=$

Total Inhibited Water Used=

dissolution with mixer (a) $=$

extra mixing (b)

total mixer running time $(a+b)=$

inhibited water addition $=$

jet transfer time $=$.

test sample \& preparation=

total time $=$

$$
210.8+2=
$$

$269.5-210.8=$

561600 gallons $=$

907.0 hrs $=$

$672.0 \mathrm{hrs}$

$842.4 \mathrm{hrs}=$

$298.7 \mathrm{brs}=$

$1200.0 \mathrm{hrs}=$

3920.1 hrs $=$
$212.8 *$ TK Lvl

58.7 "TK LvI .

160.0 "TK LV!

37.8 days

28.0 days

65.8 days

35.1 days

12.4 days

50.0 days

163.3 days

or

5.4 months

After the existing supernate transfer to TK 30 and the above four batch transfer to TK 26 , Tank 37 salt level of 212.8 inch will pass the project scope tank level of 227 inches.

Batch \#5 is shown below even though it is not required to meet the original project scope level of 227 inches. The Tank 37 level after Batch 5 will be

198.2 inches if Batch 5 is processed.

\begin{tabular}{|c|c|c|c|c|c|c|c|c|}
\hline \multirow{3}{*}{ Batch \#5 } & \multicolumn{7}{|r|}{ Step } & Hours \\
\hline & starting & water & salt & total & crystallized & total & Inhib'Water & 210.6 \\
\hline & level & added & dissolved & supernate & salt cake & content & Dissolution & 226.7 \\
\hline depth (in) & 212.83 & 40.0 & 16.67 & 58.67 & 196.17 & 254.8 & ExtraMixing & 168.0 \\
\hline gallons & 747045 & 140400 & 58500 & 205920 & 688545 & 894465 & Jet Transfer & 55.3 \\
\hline$\%$ & $83.5 \%$ & $15.7 \%$ & $6.5 \%$ & $23.0 \%$ & $77.0 \%$ & $100.0 \%$ & Sampl\&Prep. & 240.0 \\
\hline net hours & & 210.6 & 226.7 & 55.3 & transfer & batch total & 1 (days) & 37.5 \\
\hline
\end{tabular}


TANK 37H DISSOLUTION CYCLE ANALYSIS

(Case 8) One Slurry Pump with 25C Ambient Inhibited Water Water to salt dissolution ratio $=$

1 Slurry Pump with 25C Ambient IW:

5 gpm dissolution rate; dissolution \& mixing =

\begin{tabular}{|rr|r|} 
& 2.4 to 1 & $\%$ total time \\
\hline 5.2 & months total & $100 \%$ \\
$=$ & 60.5 days & $38.3 \%$ \\
\hline
\end{tabular}

a) initial salt cake level (average of $262^{\prime \prime} \& 277^{\prime \prime}$ ) $=$

b) inhibited water batch $=\sim 80 \%$ of $10 \mathrm{Kgal}$ tank $=$ inhibited water pumping to TK 37 (80 to $120 \mathrm{gpm})=$ net pumping time of one batch IW to TK $37=$ IWp $=$ number of IW batches per $12 \mathrm{hr}$-shift $=$ number of IW batches per day $=$ total time of 1 batch IW addition to TK $37=$ IWt $=$ total IW time needed/net pumping time $=$ IWt/IWp $=$ 269.50 inch 8000 gallons - 100 gpm $1.3 \mathrm{hrs}$

1 .batch per shift

2 batches per day

12 hrs

c) salt dissolution rate $=$

9

d) jet transfer rate from TK 37 to TK 30 or TK $26=$ (60 to $100 \mathrm{gpm}$ )

$5 \mathrm{gpm}$

$60 \mathrm{gpm}$

1 Slurry Pump with 25C Ambient IW:

\begin{tabular}{|c|c|c|c|c|c|c|c|c|}
\hline \multicolumn{9}{|c|}{1 Slurry Pump with 25C Ambient IW: } \\
\hline Decant & salt cake & water & salt & total liq or & crystallized & total & Inhib'Water & 0.0 \\
\hline Existifg & + dissolv. & existing & dissolved & supernate & salt cake & content & Dissolution & 0.0 \\
\hline depth (in) & 293.53 & 57.66 & 24.03 & 81.69 & 269.50 & 351.0 & ExtraMixing & 0.0 \\
\hline gallons & 1030278 & 202399 & 84333 & 286732 & 945945 & 1232010 & Jet Transfer & 77.7 \\
\hline$\%$ & $83.6 \%$ & $16.4 \%$ & $6.8 \%$ & $23.3 \%$ & $76.8 \%$ & $100.0 \%$ & Sampl\&Prep. & 240.0 \\
\hline net hours & & 0.0 & 0.0 & 77.7 & transfer & batch total & (days) & 13.2 \\
\hline
\end{tabular}

\begin{tabular}{|c|c|c|c|c|c|c|c|c|}
\hline Batch \#1 & $\begin{array}{c}\text { starting } \\
\text { level }\end{array}$ & $\begin{array}{l}\text { water } \\
\text { added }\end{array}$ & $\begin{array}{c}\text { salt } \\
\text { dissolved }\end{array}$ & $\begin{array}{c}\text { total } \\
\text { supernate }\end{array}$ & $\begin{array}{c}\text { crystallized } \\
\text { salt cake }\end{array}$ & $\begin{array}{c}\text { total } \\
\text { content }\end{array}$ & $\begin{array}{l}\text { Inhib'Water } \\
\text { Dissolution }\end{array}$ & $\begin{array}{l}210.6 \\
195.0\end{array}$ \\
\hline depth (in) & 271.50 & 40.0 & 16.67 & 58.67 & 254.83 & 313.5 & ExtraMixing & 168.0 \\
\hline gallons & 952965 & 140400 & 58500 & 205920 & 894465 & 1100385 & Jet Transfer & 55.3 \\
\hline$\%$ & $86.6 \%$ & $12.8 \%$ & $5.3 \%$ & $8.7 \%$ & $81.3 \%$ & $100.0 \%$ & Sampl\&Prep. & 240.0 \\
\hline thours & & 210.6 & 195.0 & 55.3 & transfer & batch tota & I (days) & 6.2 \\
\hline
\end{tabular}

\begin{tabular}{|c|c|c|c|c|c|c|c|c|}
\hline Batch \#2 & $\begin{array}{c}\text { starting } \\
\text { level }\end{array}$ & $\begin{array}{l}\text { water } \\
\text { added }\end{array}$ & $\begin{array}{c}\text { salt } \\
\text { dissolved }\end{array}$ & $\begin{array}{c}\text { total } \\
\text { supernate }\end{array}$ & $\begin{array}{c}\text { crystallized } \\
\text { salt cake } \\
\end{array}$ & $\begin{array}{c}\text { total } \\
\text { content. }\end{array}$ & $\begin{array}{l}\text { Inhib'Water } \\
\text { Dissolution }\end{array}$ & $\begin{array}{l}210.6 \\
195.0\end{array}$ \\
\hline depth (in) & 256.83 & 40.0 & 16.67 & 58.67 & 240.17 & 298.8 & Ext & 168.0 \\
\hline gallons & 901485 & 140400 & 58500 & $\overline{920}$ & 842985 & 104 & & 55.3 \\
\hline$\%$ & $85.9 \%$ & $13.4 \%$ & $6 \%$ & $19.6 \%$ & $80.4 \%$ & $100.0 \%$ & Sampl\&Prep. & 240.0 \\
\hline net hours & & 210.6 & 195.0 & 55.3 & transfer & batch total & (days) & 36.2 \\
\hline
\end{tabular}

\begin{tabular}{|c|c|c|c|c|c|c|c|c|}
\hline Batch $\# 3$ & $\begin{array}{c}\text { starting } \\
\text { level }\end{array}$ & $\begin{array}{l}\text { water } \\
\text { added }\end{array}$ & $\begin{array}{c}\text { salt } \\
\text { dissolved }\end{array}$ & $\begin{array}{c}\text { total } \\
\text { supernate }\end{array}$ & $\begin{array}{c}\text { crystallized } \\
\text { salt cake }\end{array}$ & $\begin{array}{c}\text { total } \\
\text { content }\end{array}$ & $\begin{array}{l}\text { Inhib'Water } \\
\text { Dissolution }\end{array}$ & $\begin{array}{l}210.6 \\
195.0\end{array}$ \\
\hline depth (in) & 242.17 & 40.0 & 16.67 & 58.67 & 225.50 & 284.2 & ExtraMixing & 168.0 \\
\hline gallons & 850005 & 140400 & 58500 & 205920 & 791505 & 997425 & Jet Transfer & 55.3 \\
\hline$\%$ & $85.2 \%$ & $14.1 \%$ & $5.9 \%$ & $20.6 \%$ & $79.4 \%$ & $100.0 \%$ & Sampl\&Prep. & 240.0 \\
\hline net hours & & 210.6 & 195.0 & 55.3 & transfer & batch tota & 1 (days) & 36.2 \\
\hline
\end{tabular}


WSKC-1K-LUU1-WL11, Rev. 0

\begin{tabular}{|c|c|c|c|c|c|c|c|c|}
\hline & & & & & & & Step & Hours \\
\hline Batch 44 & $\begin{array}{l}\text { starting } \\
\text { level }\end{array}$ & $\begin{array}{l}\text { water } \\
\text { added }\end{array}$ & $\begin{array}{c}\text { salt } \\
\text { dissolved }\end{array}$ & total & $\begin{array}{c}\text { crystallized } \\
\text { salt cake }\end{array}$ & $\begin{array}{c}\text { total } \\
\text { content }\end{array}$ & $\begin{array}{l}\text { Inhib'Water } \\
\text { Dissolution }\end{array}$ & $\begin{array}{l}210.6 \\
195.0\end{array}$ \\
\hline depth (in) & 227.50 & 40.0 & 16.67 & 58.67 & 210.83 & 269.5 & ExtraMixing & 168.0 \\
\hline gallons & 798525 & 140400 & 58500 & 205920 & 740025 & 945945 & Jet Transfer & 55.3 \\
\hline$\%$ & $84.4 \%$ & $14.8 \%$ & $6.2 \%$ & $21.8 \%$ & $78.2 \%$ & $100.0 \%$ & Sampl\&Prep. & 240.0 \\
\hline net hours & & 210.6 & 195.0 & 55.3 & transfer & batch tota & 1 (days) & $\overline{36.2}$ \\
\hline
\end{tabular}

\section{(Case 8) One Slurry Pump with 25C Ambient Inhibited Water}

Tank Level at the End of Batch $4=$

Salt Cake Level Reduced $=$

Total Inhibited Water Used $=$

dissolution with pump (a) $=$ extra pump run mixing (b) = total pump run mixing time $(a+b)=$

inhibited water addition $=$

jet transfer time $=$

test sample \& preparation $=$

total time $=$

$$
210.8+2=
$$

$269.5-210.8=$

561600 gallons $=$

780.0 hrs $=$

672.0 brs

$842.4 \mathrm{hrs}=$

298.7 brs $=$

$1200.0 \mathrm{hrs}=$

$3793.1 \mathrm{hrs}=$
212.8 "TK LVI

58.7 TK Lvl

160.0 "TK Lv1

32.5 days

28.0 days

60.5 days

35.1 days

12.4 days

50.0 days

158.0 days

5.2 months

After the existing supernate transfer to TK 30 and the above four batch transfer to TK 26 , Tank 37 salt level of 212.8 inch will pass the project scope tank level of 227 inches.

Batch \#5 is shown below even though it is not required to meet the original project scope level of 227 inches. The Tank 37 level after Batch 5 will be

198.2 inches if Batch 5 is processed.

\begin{tabular}{|c|c|c|c|c|c|c|c|c|}
\hline \multirow{3}{*}{ Batch \#5 } & \multirow{3}{*}{$\begin{array}{c}\text { starting } \\
\text { level }\end{array}$} & \multirow{3}{*}{$\begin{array}{l}\text { water } \\
\text { added }\end{array}$} & \multirow{3}{*}{$\begin{array}{c}\text { salt } \\
\text { dissolved }\end{array}$} & \multirow{3}{*}{$\begin{array}{c}\text { total } \\
\text { supernate }\end{array}$} & \multirow{3}{*}{$\begin{array}{c}\text { crystallized } \\
\text { salt cake }\end{array}$} & \multirow{3}{*}{$\begin{array}{c}\text { total } \\
\text { content }\end{array}$} & & Hours \\
\hline & & & & & & & Inhib'Water & 210.6 \\
\hline & & & & & & & Dissolution & 195.0 \\
\hline depth (in) & 212.83 & 40.0 & 16.67 & 58.67 & 196.17 & 254.8 & ExtraMixing & 168.0 \\
\hline gallons & 747045 & 140400 & 58500 & 205920 & 688545 & 894465 & Jet Transfer & 55.3 \\
\hline$\%$ & $83.5 \%$ & $15.7 \%$ & $6.5 \%$ & $23.0 \%$ & $77.0 \%$ & $100.0 \%$ & Sampl\&Prep. & 240.0 \\
\hline net hours & & 210.6 & 195.0 & 55.3 & transfer & batch total & (days) & 36.2 \\
\hline
\end{tabular}




\section{TANK 37H DISSOLUTION CYCLE ANALYSIS}

\begin{tabular}{|c|c|c|c|c|}
\hline \multicolumn{4}{|c|}{ (Case 9) Three Flygt Mixers with 25C Ambient Temperature Inhibited Water } & \multirow[b]{2}{*}{$\%$ total time } \\
\hline Water to salt dissolution ratio $=$ & 2.4 & to & 1 & \\
\hline 3 Flygt Mixers with $25 \mathrm{C}$ Ambient IW: & total & & & $100 \%$ \\
\hline $5.2 \mathrm{gpm}$ dissolution rate; dissolution \& mixing $=$ & 59.3 & days & & $37.8 \%$ \\
\hline
\end{tabular}

a) initial salt cake level (average of $262^{*} \& 277^{\prime \prime}$ ) $=$

b) inhibited water batch $=\sim 80 \%$ of $10 \mathrm{Kgal}$ tank $=$ inhibited water pumping to TK $37(80$ to $120 \mathrm{gpm})=$ net pumping time of one batch IW to TK $37=I \mathrm{Wp}=$ number of IW batches per $12 \mathrm{hr}$-shift = number of IW batches per day $=$ total time of 1 batch IW addition to TK $37=$ IWt $=$ total IW time needed/net pumping time $=I W / / W p=$

c) salt dissolution rate $=$

d) jet transfer rate from TK 37 to TK 30 or TK $26=$ (60 to $100 \mathrm{gpm}$ )
269.50 inch

8000 gallons

$100 \mathrm{gpm}$

$1.3 \mathrm{hrs}$

i batch per shift

2 batches per day

12 hrs

9

$5.2 \mathrm{gpm}$

$60 \mathrm{gpm}$

3 Flygt Mixers with 25C Ambient IW:

\begin{tabular}{|c|c|c|c|c|c|c|c|c|}
\hline & & 3 Flygt $\mathrm{Mi}$ & xers with 2 & 5C Ambient & [W: & & Step & Hours \\
\hline Decant & salt cake & water & salt & total liq or & crystallized & tot & Inhib'Water & 0.0 \\
\hline $\mathrm{Existing}$ & + dissolv. & existing & dissolved & supernate & salt cake & content & Dissolution & 0.0 \\
\hline depth (in) & 293.53 & 57.66 & 24.03 & 81.69 & 269.50 & 351.0 & ExtraMixing & 0.0 \\
\hline gallons & 1030278 & 202399 & 84333 & 286732 & 945945 & 1232010 & Jet Transfer & 77.7 \\
\hline$\%$ & $83.6 \%$ & $16.4 \%$ & $6.8 \%$ & $23.3 \%$ & $76.8 \%$ & $100.0 \%$ & Sampl\&Prep. & 240.0 \\
\hline net hours & & 0.0 & 0.0 & 77.7 & transfer & batch tota & 1 (days) & 13.2 \\
\hline
\end{tabular}

\begin{tabular}{|c|c|c|c|c|c|c|c|c|}
\hline Batch $\# 1$ & $\begin{array}{l}\text { starting } \\
\text { level }\end{array}$ & $\begin{array}{l}\text { water } \\
\text { added }\end{array}$ & $\begin{array}{c}\text { salt } \\
\text { dissolved }\end{array}$ & $\begin{array}{c}\text { total } \\
\text { supernate }\end{array}$ & $\begin{array}{c}\text { crystallized } \\
\text { salt cake }\end{array}$ & $\begin{array}{l}\text { total } \\
\text { content }\end{array}$ & $\begin{array}{l}\text { Inhib'Water } \\
\text { Dissolution }\end{array}$ & $\begin{array}{l}210.6 \\
187.5\end{array}$ \\
\hline depth (in) & 271.50 & 40.0 & 16.67 & 58.67 & 254.83 & 313.5 & ExtraMixing & 168.0 \\
\hline gallons & 952965 & 140400 & 58500 & 205920 & 894465 & 1100385 & Jet & 55.3 \\
\hline$\%$ & $86.6 \%$ & $12.8 \%$ & $5.3 \%$ & $18.7 \%$ & $81.3 \%$ & $100.0 \%$ & Sampl\&Prep. & 240.0 \\
\hline et hours & & 210.6 & 187.5 & 55.3 & transfer & batch tota & I (days) & 35.9 \\
\hline
\end{tabular}

\begin{tabular}{|c|c|c|c|c|c|c|c|c|}
\hline Batch $\# 2$ & $\begin{array}{c}\text { starting } \\
\text { level }\end{array}$ & $\begin{array}{l}\text { water } \\
\text { added }\end{array}$ & $\begin{array}{c}\text { salt } \\
\text { dissolved }\end{array}$ & $\begin{array}{c}\text { total } \\
\text { supernate }\end{array}$ & $\begin{array}{l}\text { crystallized } \\
\text { salt cake }\end{array}$ & $\begin{array}{c}\text { total } \\
\text { content }\end{array}$ & $\begin{array}{l}\text { Inhib'Water } \\
\text { Dissolution }\end{array}$ & $\begin{array}{l}210.6 \\
187.5\end{array}$ \\
\hline depth (in) & 256.83 & 40.0 & 16.67 & 58.67 & 240.17 & 298.8 & ExtraMixing & 168.0 \\
\hline gallons & 901485 & 140400 & 58500 & 205920 & 842985 & 1048905 & Jet Transfer & 55.3 \\
\hline$\%$ & $85.9 \%$ & $13.4 \%$ & $5.6 \%$ & $19.6 \%$ & $80.4 \%$ & $100.0 \%$ & Sampl\&Prep. & 240.0 \\
\hline net hours & & 210.6 & 187.5 & 55.3 & transfer & batch tota & (days) & 35.9 \\
\hline
\end{tabular}

\begin{tabular}{|c|c|c|c|c|c|c|c|c|}
\hline Batch \#3 & $\begin{array}{c}\text { starting } \\
\text { level }\end{array}$ & $\begin{array}{l}\text { water } \\
\text { added }\end{array}$ & $\begin{array}{c}\text { salt } \\
\text { dissolved }\end{array}$ & $\begin{array}{c}\text { total } \\
\text { supernate }\end{array}$ & $\begin{array}{l}\text { crystallized } \\
\text { salt cake }\end{array}$ & $\begin{array}{c}\text { total } \\
\text { content }\end{array}$ & Inhib'Water & $\begin{array}{l}210.6 \\
187.5\end{array}$ \\
\hline depth (in) & 242.17 & 40.0 & 16.67 & 58.67 & 225.50 & 284.2 & ExtraMixing & 168.0 \\
\hline gallons & 850005 & 140400 & 58500 & 205920 & 791505 & 997425 & Jet Transfer & 55.3 \\
\hline$\%$ & $85.2 \%$ & $14.1 \%$ & $5.9 \%$ & $20.6 \%$ & $79.4 \%$ & $100.0 \%$ & Sampl\&Prep. & 240.0 \\
\hline net hours & & 210.6 & 187.5 & 55.3 & transfer & batch tota & 1 (days) & 35.9 \\
\hline
\end{tabular}




\begin{tabular}{|c|c|c|c|c|c|c|c|c|}
\hline & & & & & & & Step & Hours \\
\hline Batch \#4 & $\begin{array}{l}\text { starting } \\
\text { level }\end{array}$ & water & salt & total & crystallized & & Inhib'Water & 210.6 \\
\hline & & & dissolved & & salt cake & content & Diss & 187.5 \\
\hline depth (in) & 227.50 & 40.0 & 16.67 & 58.67 & 210.83 & 269.5 & ExtraMixing & 168.0 \\
\hline gallons & 798525 & 140400 & 58500 & 205920 & 740025 & 945945 & Jet Transfer & 55.3 \\
\hline$\%$ & $84.4 \%$ & $14.8 \%$ & $6.2 \%$ & $21.8 \%$ & $78.2 \%$ & $100.0 \%$ & Sampl\&Prep. & 240.0 \\
\hline \multicolumn{2}{|l|}{ net hours } & 210.6 & 187.5 & 55.3 & transfer & batch total & (days) & 35.9 \\
\hline
\end{tabular}

\section{(Case 9) Three Flygt Mixers with 25C Ambient Temperature Inhibited Wate}

Tank Level at the End of Batch $4=$

Salt Cake Level Reduced $=$

Total Inhibited Water Used=

dissolution with mixer (a) $=$

extra mixing (b)

total mixer running time $(a+b)=$

inhibited water addition $=$

jet transfer time $=$

test sample \& preparation=

total time $=$

$$
210.8+2=
$$

$$
269.5-210.8=
$$

561600 gallons =

750.0 hrs $=$

$672.0 \mathrm{brs}$
212.8 " TK LVI

58.7 " TK LVI

160.0 "TK LvI

31.3 days

28.0 days

59.3 days

35.1 days

12.4 days

$1200.0 \mathrm{hrs}=$

50.0 days

$3763.1 \mathrm{brs}=$

156.8 days

or

5.1 months

After the existing supernate transfer to TK 30 and the above four batch transfer to TK 26,

Tank 37 salt level of 212.8 inch will pass the project scope tank level of 227 inches.

Batch $\# S$ is shown below even though it is not required to meet the original project scope level of 227 inches. The Tank 37 level after Batch 5 will be

\begin{tabular}{|c|c|c|c|c|c|c|c|c|}
\hline & & & & & & & Step & Iours \\
\hline 3 atch 45 & starting & water & salt & total & crystallized & & Inhib'Water & 210.6 \\
\hline & level & added & dissolved & pernate & salt cake & content & Diss & 187.5 \\
\hline depth (in & 212.83 & 40.0 & 16.67 & 58.67 & 196.17 & 254.8 & ExtraM & 168.0 \\
\hline gallons & 747045 & 140400 & 58500 & 20 & 45 & 894465 & Jet Tran & 55.3 \\
\hline$\%$ & $83.5 \%$ & $15.7 \%$ & $q_{0}$ & $23.0 \%$ & $0 \%$ & $0 \%$ & Sampl\&Prep. & 240.0 \\
\hline t hous & & 210.6 & 187.5 & 55.3 & fer & batch tota & 1 (days) & 35.9 \\
\hline
\end{tabular}

198.2 inches if Batch 5 is processed. 


\section{TANK 37H DISSOLUTION CYCLE ANALYSIS}

\begin{tabular}{|lr|r|}
\hline $\begin{array}{l}\text { (Case 10) One Flygt Mixer with 75C Heated Inhibited Water } \\
\text { Water to salt dissolution ratio }=\end{array}$ & 2.4 to 1 & $\%$ total time \\
\hline $\begin{array}{r}\text { 1 Flygt Mixer with 50C Heated IW: } \\
\text { 5.6 gpm dissolution rate; dissolution \& mixing }=\end{array}$ & 57.0 days & $100 \%$ \\
\hline
\end{tabular}

a) initial salt cake level (average of $262^{\prime \prime} \& 277^{\prime}$ ) =

b) inhibited water batch $=\sim 80 \%$ of $10 \mathrm{Kgal}$ tank $=$ inhibited water pumping to TK 37 (80 to $120 \mathrm{gpm})=$ net pumping time of one batch IW to TK $37=\mathrm{IWp}=$ number of IW batches per $12 \mathrm{hr}$-shift $=$ number of IW batches per day $=$ total time of 1 batch IW addition to TK $37=I W \mathrm{~T}=$ total IW time needed/net pumping time $=$ IWt/IWp $=$

c) salt dissolution rate $=$

d) jet transfer rate from TK 37 to TK 30 or TK $26=$ (60 to $100 \mathrm{gpm}$ )
269.50 inch

8000 gallons

$100 \mathrm{gpm}$

1.3 hrs

1 batch per shift

2 batches per day

12 hrs

9

$5.6 \mathrm{gpm}$

$60 \mathrm{gpm}$

1 Flygt Mixer with 50C Heated IW:

\begin{tabular}{|c|c|c|c|c|c|c|c|c|}
\hline & & 1 Flygt Mi & xer with 50 & $C$ Heated IW & & & Step & ours \\
\hline Decant & salt cake & water & salt & total liq or & crysta & tot & Inhib'Water & 0.0 \\
\hline Existing & $v$. & isting & dissolved & supernate & & content & Dis & 0.0 \\
\hline depth & 3.53 & 57.66 & 4.03 & 81.69 & 50 & 351.0 & Extr & 0.0 \\
\hline gallons & 030278 & 202399 & 84333 & 86732 & 15 & 10 & Jet $T$ & 77.7 \\
\hline$\%$ & $.6 \%$ & $6.4 \%$ & $6.8 \%$ & $23.3 \%$ & $76.8 \%$ & $100.0 \%$ & Sampl\&Prep. & 240.0 \\
\hline et hours & & 0.0 & 0.0 & 77.7 & transfer & 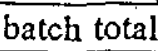 & (s) & 3.2 \\
\hline
\end{tabular}

\begin{tabular}{|c|c|c|c|c|c|c|c|c|}
\hline Batch \#1 & $\begin{array}{c}\text { starting } \\
\text { level }\end{array}$ & $\begin{array}{l}\text { water } \\
\text { added }\end{array}$ & $\begin{array}{c}\text { salt } \\
\text { dissolved }\end{array}$ & $\begin{array}{c}\text { total } \\
\text { supernate }\end{array}$ & $\begin{array}{l}\text { crystallized } \\
\text { salt cake }\end{array}$ & $\begin{array}{c}\text { total } \\
\text { content }\end{array}$ & $\begin{array}{l}\text { Inhib'Water } \\
\text { Dissolution }\end{array}$ & $\begin{array}{l}210.6 \\
174.1\end{array}$ \\
\hline depth (in) & 271.50 & 40.0 & 16.67 & 58.67 & 254.83 & 313.5 & Extral & 168.0 \\
\hline gallons & 952965 & 140400 & 58500 & 205920 & 894465 & 1100385 & Jet Transfer & 55.3 \\
\hline$\%$ & $86.6 \%$ & $12.8 \%$ & $5.3 \%$ & $18.7 \%$ & $81.3 \%$ & $100.0 \%$ & Sampl\& & 240.0 \\
\hline net hours & & 210.6 & 174.1 & 55.3 & transfer & batch tota & 1 (days) & 35.3 \\
\hline
\end{tabular}

\begin{tabular}{|c|c|c|c|c|c|c|c|c|}
\hline Batch $\# 2$ & $\begin{array}{c}\text { starting } \\
\text { level }\end{array}$ & $\begin{array}{l}\text { water } \\
\text { added }\end{array}$ & $\begin{array}{c}\text { salt } \\
\text { dissolved }\end{array}$ & $\begin{array}{c}\text { total } \\
\text { supernate }\end{array}$ & $\begin{array}{l}\text { crystallized } \\
\text { salt cake }\end{array}$ & $\begin{array}{c}\text { total } \\
\text { content }\end{array}$ & $\begin{array}{l}\text { Inhib'Water } \\
\text { Dissolution }\end{array}$ & $\begin{array}{l}210.6 \\
174.1\end{array}$ \\
\hline depth (in) & 256.83 & 40.0 & 16.67 & 58.67 & 240.17 & 298.8 & ExtraMixing & 168.0 \\
\hline gallons & 901485 & 140400 & 58500 & 205920 & 842985 & 1048905 & Jet Tranisfer & 55.3 \\
\hline$\%$ & $85.9 \%$ & $13.4 \%$ & $5.6 \%$ & $19.6 \%$ & $80.4 \%$ & $100.0 \%$ & Sampl\&Prep. & 240.0 \\
\hline net hours & & 210.6 & 174.1 & 55.3 & transfer & batch total & (days) & 35.3 \\
\hline
\end{tabular}

\begin{tabular}{|c|c|c|c|c|c|c|c|c|}
\hline Batch $\# 3$ & $\begin{array}{c}\text { starting } \\
\text { level }\end{array}$ & $\begin{array}{l}\text { water } \\
\text { added }\end{array}$ & $\begin{array}{c}\text { salt } \\
\text { dissolved }\end{array}$ & $\begin{array}{c}\text { total } \\
\text { supernate }\end{array}$ & $\begin{array}{l}\text { crystallized } \\
\text { salt cake }\end{array}$ & $\begin{array}{c}\text { total } \\
\text { content }\end{array}$ & $\begin{array}{l}\text { Inhib'Water } \\
\text { Dissolution }\end{array}$ & $\begin{array}{l}210.6 \\
174.1\end{array}$ \\
\hline depth (in) & 242.17 & 40.0 & 16.67 & 58.67 & 225.50 & 284.2 & ExtraMixing & 168.0 \\
\hline gallons & 850005 & 140400 & 58500 & 205920 & 791505 & 997425 & Jet Transfer & 55.3 \\
\hline $\mathscr{F}_{0}$ & $85.2 \%$ & $14.1 \%$ & $5.9 \%$ & $20.6 \%$ & $79.4 \%$ & $100.0 \%$ & Sampl\&\&Prep. & 240.0 \\
\hline net hours & & 210.6 & 174.1 & 55.3 & transfer & batch total & 1 (days) & 35.3 \\
\hline
\end{tabular}




\section{(Case 10) One Flygt Mixer with 75C Heated Inhibited Water}

Tank Level at the End of Batch $4=$

Salt Cake Level Reduced $=$

Total Inhibited Water Used=

dissolution with mixer $(a)=$ extra mixing (b)

total mixer running time $(a+b)=$

inhibited water addition $=$

jet transfer time $=$.

test sample \& preparation $=$

total time $=$

$$
210.8+2=
$$$$
269.5-210.8=
$$

561600 gallons =

$696.4 \mathrm{hrs}=$

$672.0 \mathrm{brs}$

$842.4 \mathrm{hrs}=$

298.7 hrs $=$

$1200.0 \mathrm{hrs}=$

$3709.5 \mathrm{hrs}=$
212.8 "TK Lvl

58.7 " TK Lvl

160.0 * TK LvI

29.0 days

28.0 days

57.0 days

35.1 days

12.4 days

50.0 days

154.6 days

After the existing supernate transfer to TK 30 and the above four batch transfer to TK 26,

Tank 37 salt level of 212.8 inch will pass the project scope tank level of 227 inches.

Batch $\not 5$ is shown below even though it is not required to meet the original project scope level of 227 inches. The Tank 37 level after Batch 5 will be

198.2 inches if Batch 5 is processed.

\begin{tabular}{|c|c|c|c|c|c|c|c|c|}
\hline & & & & & & & Step & Hours \\
\hline Batch \#5 & $\begin{array}{l}\text { starting } \\
\text { level }\end{array}$ & water & salt & total & crystallized & total & Inhib'Water & 210.6 \\
\hline depth (in) & 212.83 & 40.0 & 16.67 & 58.67 & 196.17 & 254.8 & ExtraMixing & 168.0 \\
\hline gallons & 747045 & 140400 & 58500 & 205920 & 688545 & 894465 & Jet Transfer & 55.3 \\
\hline$\%$ & $83.5 \%$ & $15.7 \%$ & $6.5 \%$ & $23.0 \%$ & $77.0 \%$ & $100.0 \%$ & Sampl\&Prep. & 240.0 \\
\hline net hours & & 210.6 & 174.1 & 55.3 & transfer & batch tota & 1 (days) & 35.3 \\
\hline
\end{tabular}




\section{TANK 37H DISSOLUTION CYCLE ANALYSIS}

\begin{tabular}{|c|c|c|c|c|}
\hline $\begin{array}{l}\text { Case 11) Two Flygt Mixers with soc Heated Inhibited Water } \\
\text { Water to sait dissolution ratio }=\end{array}$ & 2.4 & to & I & $\%$ total time \\
\hline Flygt Mixe & total & & & $100 \%$ \\
\hline $6.1 \mathrm{gpm}$ dissolution rate; dissolution \& mixing = & 54.6 & days & & $36 \%$ \\
\hline
\end{tabular}

a) initial salt cake level (average of $262^{\prime \prime} \& .277^{\prime \prime}$ ) =

269.50 inch

b) inhibited water batch $=\sim 80 \%$ of $10 \mathrm{Kgal}$ tank $=$

8000 gallons

inhibited water pumping to TK 37 (80 to $120 \mathrm{gpm}$ ) =

$100 \mathrm{gpm}$

net pumping time of one batch IW to TK $37=\mathrm{IWp}=$

1.3 hrs

number of IW batches per $12 \mathrm{hr}$-6hift $=$

1 batch per shift

number of IW batches per day $=$

total time of 1 batch IW addition to TK $37=$ IWt $=$

2 batches per day

total IW time needed/net pumping time $=\mathrm{IWt} / \mathrm{IWp}=$

12 brs

c) salt dissolution rate $=$

$6.1 \mathrm{gpm}$

d) jet transfer rate from TK 37 to TK 30 or TK $26=$ (60 to $100 \mathrm{gpm}$ )

$60 \mathrm{gpm}$

2 Flygt Mixers with 50C Heated IW:

\begin{tabular}{|c|c|c|c|c|c|c|c|c|}
\hline \multicolumn{9}{|c|}{ Hone } \\
\hline Decan & salt cake & water & salt & total liq or & crystallized & total & Inhib'Water & 0.0 \\
\hline Existig & + dissolv. & existing & dissolved & supernate & salt & content & Dissolution & 0.0 \\
\hline depth (in) & 293.53 & 57.66 & 24.03 & 81.69 & 269.50 & 351.0 & ExtraMixing & 0.0 \\
\hline gallons & 1030278 & 202399 & 84333 & 286732 & 945945 & 1232010 & Jet.Transfer & 77.7 \\
\hline$\%$ & $83.6 \%$ & $16.4 \%$ & $6.8 \%$ & $23.3 \%$ & $76.8 \%$ & $100.0 \%$ & Sampl\&Prep. & 240.0 \\
\hline net hours & & 0.0 & 0.0 & 77.7 & transfer & batch total & (days) & 13.2 \\
\hline
\end{tabular}

\begin{tabular}{|c|c|c|c|c|c|c|c|c|}
\hline Batch \#1 & $\begin{array}{c}\text { starting } \\
\text { level }\end{array}$ & $\begin{array}{l}\text { water } \\
\text { added }\end{array}$ & $\begin{array}{c}\text { salt } \\
\text { dissolved }\end{array}$ & $\begin{array}{c}\text { total } \\
\text { supernate }\end{array}$ & $\begin{array}{l}\text { crystallized } \\
\text { salt cake }\end{array}$ & $\begin{array}{c}\text { total } \\
\text { content }\end{array}$ & $\begin{array}{l}\text { Inhib'Water } \\
\text { Dissolution }\end{array}$ & $\begin{array}{l}210.6 \\
159.8\end{array}$ \\
\hline depth (in) & 271.50 & 40.0 & 16.67 & 58.67 & 254.83 & 313.5 & ExtraMixing & 168.0 \\
\hline gallons & 952965 & 140400 & 58500 & 205920 & 894465 & 1100385 & Jet Transfer & 55.3 \\
\hline$\%$ & $86.6 \%$ & $12.8 \%$ & $5.3 \%$ & $18.7 \%$ & $81.3 \%$ & $100.0 \%$ & Sampl\&Prep. & 240.0 \\
\hline net hours & & 210.6 & 159.8 . & 55.3 & transfer & batch tota & (days) & 34.7 \\
\hline
\end{tabular}

\begin{tabular}{|c|c|c|c|c|c|c|c|c|}
\hline Batch \#2 & $\begin{array}{c}\text { starting } \\
\text { level }\end{array}$ & $\begin{array}{l}\text { water } \\
\text { added }\end{array}$ & $\begin{array}{c}\text { salt } \\
\text { dissolved }\end{array}$ & $\begin{array}{c}\text { total } \\
\text { supernate }\end{array}$ & $\begin{array}{l}\text { crystallized } \\
\text { salt cake }\end{array}$ & $\begin{array}{c}\text { total } \\
\text { content }\end{array}$ & $\begin{array}{l}\text { Inhib Water } \\
\text { Dissolution }\end{array}$ & $\begin{array}{l}210.6 \\
159.8\end{array}$ \\
\hline depth (in) & 256.83 & 40.0 & 16.67 & 58.67 & 240.17 & 298.8 & ExtraMixing & 168.0 \\
\hline gallons & 901485 & 140400 & 58500 & 205920 & 842985 & 1048905 & Jet Transfer & 55.3 \\
\hline$\%$ & $85.9 \%$ & $13.4 \%$ & $5.6 \%$ & $19.6 \%$ & $80.4 \%$ & $100.0 \%$ & Sampl\&Prep. & 240.0 \\
\hline net hours & & 210.6 & 159.8 & 55.3 & transfer & batch tota & (days) & 34.7 \\
\hline
\end{tabular}

\begin{tabular}{|c|c|c|c|c|c|c|c|c|}
\hline Batch $\not 3$ & $\begin{array}{l}\text { starting } \\
\text { level }\end{array}$ & $\begin{array}{l}\text { water } \\
\text { added }\end{array}$ & $\begin{array}{c}\text { salt } \\
\text { dissolved }\end{array}$ & $\begin{array}{c}\text { total } \\
\text { supernate }\end{array}$ & $\begin{array}{l}\text { crystallized } \\
\text { salt cake }\end{array}$ & $\begin{array}{l}\text { total } \\
\text { content }\end{array}$ & $\begin{array}{l}\text { Inhib'Water } \\
\text { Dissolution }\end{array}$ & $\begin{array}{l}210.6 \\
159.8\end{array}$ \\
\hline depth (in) & 242.17 & 40.0 & 16.67 & 58.67 & 225.50 & 284.2 & ExtraMixing & 168.0 \\
\hline gallons & 850005 & 140400 & 58500 & 205920 & 791505 & 997425 & Jet Transfer & 55.3 \\
\hline$\%$ & $85.2 \%$ & $14.1 \%$ & $5.9 \%$ & $20.6 \%$ & $79.4 \%$ & $100.0 \%$ & Sampl\&Prep. & 240.0 \\
\hline net hours & & 210.6 & 159.8 & 55,3 & transfer & batch tota & 1 (days) & 34.7 \\
\hline
\end{tabular}




\begin{tabular}{|c|c|c|c|c|c|c|c|c|}
\hline & & & & & & & Step & Hours \\
\hline Batch $\# 4$ & starting & water & salt & total & crystallized & total & Inhib'Water & 210.6 \\
\hline & level & added & dissolved & superate & salt cake & content & Dissolution & 159.8 \\
\hline depth (in) & 227.50 & 40.0 & 16.67 & 58.67 & 210.83 & 269.5 & ExtraMixing & 168.0 \\
\hline gallons & 798525 & 140400 & 58500 & 205920 & 740025 & 945945 & Jet Transfer & 55.3 \\
\hline$\%$ & $84.4 \%$ & $14.8 \%$ & $6.2 \%$ & $21.8 \%$ & $78.2 \%$ & $100.0 \%$ & Sampl\&Prep. & 240.0 \\
\hline \multicolumn{2}{|l|}{ net hours } & 210.6 & 159.8 & 55.3 & transfer & batch total & (days) & 34.7 \\
\hline
\end{tabular}

\section{(Case 11) Two Flygt Mixers with 50C Heated Inhibited Water}

Tank Level at the End of Batch $4=$

Salt Cake Level Reduced $=$

Total Inhibited Water Used=

dissolution with mixer $(a)=$ extra mixing (b).

total mixer running time $(a+b)=$

inhibited water addition $=$

jet transfer time $=$.

test sample \& preparation=

total time $=$

$$
210.8+2=
$$

269.5

$-210.8=$

561600 gallons

$639.3 \mathrm{hrs}=$

$672.0 \mathrm{hrs}$

$842.4 \mathrm{hrs}=$

298.7 hrs $=$

$1200.0 \mathrm{hrs}=$

3652.4 hrs $=$
212.8 "TK Lvl

$58.7 *$ TK Lvl

160.0 "TK Lvl

26.6 days

28.0 days

54.6 days

35.1 days

12.4 days

50.0 days

152.2 days

or

5.0 months

After the existing supernate transfer to TK 30 and the above four batch transfer to TK 26, Tank 37 salt level of 212.8 inch will pass the project scope tank level of 227 inches.

Batch \#5 is shown below cven though it is not required to meet the original project scope level of 227 inches. The Tank 37 level after Batch 5 will be

198.2 inches if Batch 5 is processed.

\begin{tabular}{|c|c|c|c|c|c|c|c|c|}
\hline & & & & & & & Step & Hours. \\
\hline Batch \#5 & starting & water & salt & otal & crystalli & total & Inhib'Water & 210.6 \\
\hline & level & added & dissolved & supernate & salt cake & content & Dissolution & 159.8 \\
\hline depth (in) & 212.83 & 40.0 & 16.67 & 58.67 & 196.17 & 254.8 & ExtraMixing & 168.0 \\
\hline gallons & 747045 & 140400 & 58500 & 205920 & 688545 & 894465 & Jet Transfer & 55.3 \\
\hline$\%$ & $83.5 \%$ & $15.7 \%$ & $6.5 \%$ & $23.0 \%$ & $77.0 \%$ & $100.0 \%$ & Sampl\&Prep. & 240.0 \\
\hline net bours & & 210.6 & 159.8 & 55.3 & ransfer & batch total & (days) & 34.7 \\
\hline
\end{tabular}




\section{TANK 37H DISSOLUTION CYCLE ANALYSIS}

(Case 12) One Slurry Pump with 50C Heated Inhibited Water Water to salt dissolution ratio $=$

1 Slurry Pump with 50C Heated IW:

7.1 gpm dissolution rate; dissolution \& mixing =

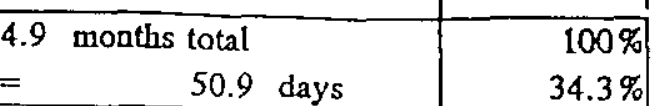

a) initial salt cake level (average of $262^{*} \& 277^{\star}$ ) $=$

b) inhibited water batch $=\sim 80 \%$ of $10 \mathrm{Kgal} \mathrm{tank}=$ inhibited water pumping to TK $37(80$ to $120 \mathrm{gpm})=$ net pumping time of one batch IW to TK $37=I \mathrm{Wp}=$ number of IW batches per $12 \mathrm{hr}$-shift = number of IW batches per day = total time of 1 batch IW addition to TK $37=$ IWt $=$ total IW time needed/net pumping time $=\mathrm{IW} / \mathrm{IWp}=$

269.50 inch 8000 gallons

$100 \mathrm{gpm}$

1.3 . hrs

1 batch per shift

2 batches per day

$12 \mathrm{hrs}$

c) salt dissolution tate $=$

9

d) jet transfer rate from TK 37 to TK 30 or TK $26=$ (60 to $100 \mathrm{gpm}$ )

\section{$7.1 \mathrm{gpm}$ \\ $60 \mathrm{gpm}$}

1 Slurry Pump with 50C Heated IW:

\begin{tabular}{|c|c|c|c|c|c|c|c|c|}
\hline \multicolumn{9}{|c|}{1 Slurry Pump with 50C Heated IW: } \\
\hline Decant & salt cake & water & salt & total liq or & crystallized & total & Inhib'Water & 0.0 \\
\hline Bxs $1+1$ & + dissolv. & existing & dissolved & supernate & salt cake & content & Dissolution & 0.0 \\
\hline depth (in) & 293.53 & 57.66 & 24.03 & 81.69 & 269.50 & 351.0 & ExtraMixing & 0.0 \\
\hline gallons & 1030278 & 202399 & 84333 & 286732 & 945945 & 1232010 & Jet Transfer & 77.7 \\
\hline$\%$ & $83.6 \%$ & $16.4 \%$ & $6.8 \%$ & $23.3 \%$ & $76.8 \%$ & $100.0 \%$ & Sampl\&Prep. & 240.0 \\
\hline net hours & & 0.0 & 0.0 & 77.7 & transfer & batch tota & (days) & 13.2 \\
\hline
\end{tabular}

\begin{tabular}{|c|c|c|c|c|c|c|c|c|}
\hline Batch $\not 1$ & $\begin{array}{c}\text { starting } \\
\text { level }\end{array}$ & $\begin{array}{l}\text { water } \\
\text { added }\end{array}$ & $\begin{array}{l}\text { salt } \\
\text { issolye }\end{array}$ & total & crystallized & total & |Inhib'Water & 210.6 \\
\hline depth (in) & 271.50 & & & Jernate & & content & & 137 \\
\hline gallons & 952965 & U. & 57 & 58.67 & 254.83 & 313.5 & Extra & 168.0 \\
\hline$\%$ & & 40400 & 58500 & 205920 & 894465 & 1100385 & Jet Transfer & 55.3 \\
\hline \multirow{2}{*}{\multicolumn{2}{|c|}{ net hours }} & $2.8 \%$ & $5.3 \%$ & $18.7 \%$ & $81.3 \%$ & $100.0 \%$ & Sampl\&Prep. & 240.0 \\
\hline & & & 137.3 & 55.3 & transfer & batch total & 1 (days) & 33.8 \\
\hline
\end{tabular}

\begin{tabular}{|c|c|c|c|c|c|c|c|c|}
\hline Batch \# & $\begin{array}{c}\text { starting } \\
\text { level }\end{array}$ & $\begin{array}{l}\text { water } \\
\text { added }\end{array}$ & $\begin{array}{c}\text { salt } \\
\text { dissolved }\end{array}$ & $\begin{array}{c}\text { total } \\
\text { supernate }\end{array}$ & $\begin{array}{c}\text { crystallized } \\
\text { salt cake }\end{array}$ & $\begin{array}{c}\text { total } \\
\text { content }\end{array}$ & $\begin{array}{l}\text { Inhib'Water } \\
\text { Dissolution }\end{array}$ & $\begin{array}{l}210.6 \\
137.3\end{array}$ \\
\hline depth (in) & 256.83 & 40.0 & 16.67 & 58.67 & 240.17 & 298.8 & BxtraMixing & 168.0 \\
\hline gallons & 901485 & 140400 & 58500 & 205920 & 842985 & 1048905 & Jet' & 55.3 \\
\hline$\%$ & $85.9 \%$ & $13.4 \%$ & $.6 \%$ & $19.6 \%$ & $80.4 \%$ & $100.0 \%$ & Sampi\&Prep. & 240.0 \\
\hline & & 210.6 & 137.3 & $55: 3$ & transfer & batch total & 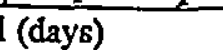 & 33.8 \\
\hline
\end{tabular}

\begin{tabular}{|c|c|c|c|c|c|c|c|c|}
\hline Batch $\$ 3$ & $\begin{array}{c}\text { starting } \\
\text { level }\end{array}$ & $\begin{array}{l}\text { water } \\
\text { added }\end{array}$ & $\begin{array}{c}\text { salt } \\
\text { dissolved }\end{array}$ & $\begin{array}{c}\text { total } \\
\text { supernate }\end{array}$ & $\begin{array}{c}\text { crystallized } \\
\text { salt cake }\end{array}$ & $\begin{array}{c}\text { total } \\
\text { content }\end{array}$ & $\begin{array}{l}\text { Inhib'Water } \\
\text { Dissolution }\end{array}$ & $\begin{array}{l}210.6 \\
137.3\end{array}$ \\
\hline depth (in) & 242.17 & 40.0 & 16.67 & 58.67 & 225.50 & 284.2 & Extr & 168.0 \\
\hline gallons & 850005 & 140400 & 58500 & 205920 & 791505 & 997425 & Jet & 55.3 \\
\hline$\%$ & $85.2 \%$ & $14.1 \%$ & $5.9 \%$ & $20.6 \%$ & $79.4 \%$ & $0 \%$ & Sampl\& & 240.0 \\
\hline net hours & & 210.6 & 137.3 & 55.3 & transfer & batch tota & 1 (days) & 33.8 \\
\hline
\end{tabular}




\begin{tabular}{|c|c|c|c|c|c|c|c|c|}
\hline & & & & & & & Step & Hours \\
\hline Batch $\$ 4$ & $\begin{array}{l}\text { starting } \\
\text { level }\end{array}$ & $\begin{array}{l}\text { water } \\
\text { sdded }\end{array}$ & $\begin{array}{c}\text { salt } \\
\text { dissolyed }\end{array}$ & total & crystallized & total & $\begin{array}{l}\text { Inhib'Water } \\
\text { Dissolution }\end{array}$ & 210.6 \\
\hline depth (in) & 227.50 & 40.0 & 16.67 & 58.67 & 210.83 & 269.5 & ExtraMixing & 168.0 \\
\hline gallons & 798525 & 140400 & 58500 & 205920 & 740025 & 945945 & Jet Transfer & 55.3 \\
\hline$\%$ & $84.4 \%$ & $14.8 \%$ & $6.2 \%$ & $21.8 \%$ & $78.2 \%$ & $100.0 \%$ & Sampl\&Prep. & 240.0 \\
\hline \multicolumn{2}{|l|}{ net hours } & 210.6 & 137.3 & 55.3 & transfer & batch total & (days) & 33.8 \\
\hline
\end{tabular}

\section{(Case 12) One Slurry Pump with 50C Heated Inhibited Water}

Tank Level at the End of Batch $4=$

Salt Cake Level Reduced $=$

Total Inhibited Water Used=

dissolution with pump (a) $=$ extra pump run mixing (b) =

total pump run mixing time $(a+b)=$

inhibited water addition $=$

jet transfer time $=$

test sample \& preparation=

total time $=$

$$
210.8+2=
$$

269.5

$-210.8=$

561600 gallons =

$549.3 \mathrm{hrs}=$

$672.0 \mathrm{hrs}$

$842.4 \mathrm{hrs}=$

$298.7 \mathrm{hrs}=$

$1200.0 \mathrm{hrs}=$

$3562.4 \mathrm{hrs}=$
212.8 "TK Lvl

58.7 " TK Lv1

160.0 "TK Lvl

22.9 days

28.0 days

50.9 days

35.1 days

12.4 days

50.0 days

148.4 days

4.9 months

After the existing supernate transfer to TK 30 and the above four batch transfer to TK 26, Tank 37 salt level of 212.8 inch will pass the project scope tank level of 227 inches.

Batch \#5 is shown below even though it is not required to meet the original project scope level of 227 inches. The Tank 37 level after Batch 5 will be

198.2 inches if Batch 5 is processed.

\begin{tabular}{|c|c|c|c|c|c|c|c|c|}
\hline \multirow{3}{*}{ Batch \#5 } & \multirow{3}{*}{$\begin{array}{l}\text { starting } \\
\text { level }\end{array}$} & \multirow{3}{*}{$\begin{array}{l}\text { water } \\
\text { added }\end{array}$} & \multirow{3}{*}{\begin{tabular}{|c|} 
salt \\
dissolved
\end{tabular}} & \multirow{3}{*}{$\begin{array}{c}\text { total } \\
\text { suppernate }\end{array}$} & \multirow{3}{*}{$\begin{array}{c}\text { crystallized } \\
\text { salt cake }\end{array}$} & \multirow{3}{*}{$\begin{array}{c}\text { total } \\
\text { content }\end{array}$} & Step & \multirow{2}{*}{$\frac{\text { Hours }}{210.6}$} \\
\hline & & & & & & & Inhib'Water & \\
\hline & & & & & & & Dissolution & 137.3 \\
\hline depth (in) & 212.83 & 40.0 & 16.67 & 58.67 & 196.17 & 254.8 & ExtraMixing & 168.0 \\
\hline gallons & 747045 & 140400 & 58500 & 205920 & 688545 & 894465 & Jet Transfer & 55.3 \\
\hline$\%$ & $83 . \overline{5 \%}$ & $15.7 \%$ & $6.5 \%$ & $23: 0 \%$ & $77.0 \%$ & $100.0 \%$ & Sampl\&Prep. & 240.0 \\
\hline net hours & & 210.6 & 137.3 & 55.3 & transfer & batch total & (days) & 33.8 \\
\hline
\end{tabular}




\section{TANK 37H DISSOLUTION CYCLE ANALYSIS}

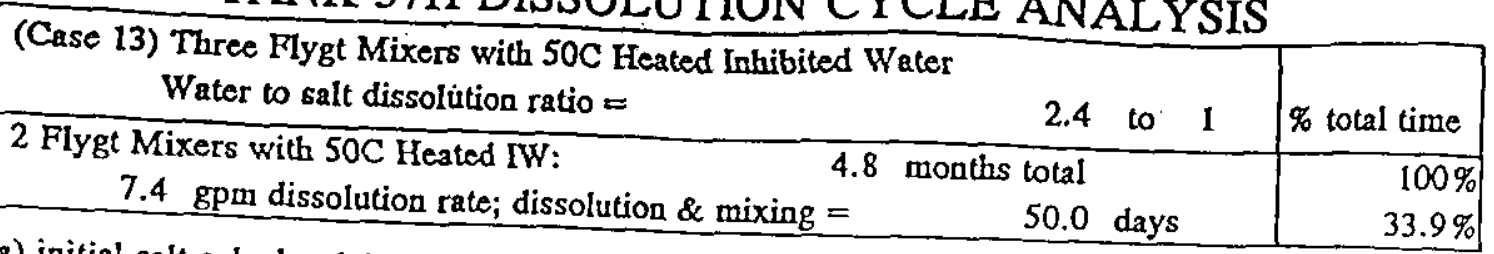

a) initial salt cake level (average of 262" \& 277") =

b) inbibited water batch $=\sim 80 \%$ of $10 \mathrm{Kgal} \mathrm{tank}=$ inhibited water pumping to TK 37 (80 to $120 \mathrm{gpm})=$ net pumping time of one batch IW to TK $37=$ IWp $=$ number of IW batches per $12 \mathrm{hr}$-shift $=$ number of $T W$ batches per day = total time of 1 batch IW addition to TK $37=$ IWt $=$ total IW time needed/net pumping time $=$ IWt/IWp $=$

c) salt dissolution rate $=$

d) jet transfer rate from TK 37 to TK 30 or TK $26=$ (60 to $100 \mathrm{gpm}$ )

$$
\begin{aligned}
269.50 & \text { inch } \\
8000 & \text { gallons } \\
100 & \text { gpm } \\
1.3 & \text { hrs } \\
1 & \text { batch per shift } \\
2 & \text { batches per day } \\
12 & \text { brs } \\
9 &
\end{aligned}
$$

\begin{tabular}{|c|c|c|c|c|c|c|c|c|}
\hline Decar & & & 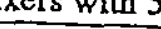 & $C$. Heated I & & & Step & Hours \\
\hline Existing. & $\begin{array}{l}\text { salt cake } \\
+ \text { dissolv. }\end{array}$ & $\begin{array}{l}\text { water } \\
\text { existing }\end{array}$ & salt & total liq or & crystallized & tot & Inhib'Water & 0.0 \\
\hline depth (in) & 293.53 & & alssolved & supernate & salt cake & content & Dissolution & 0.0 \\
\hline gallons & $\frac{293.33}{1030278}$ & 57.66 & 24.03 & 81.69 & 269.50 & 351.0 & ExtraMixing & 0.0 \\
\hline$\%$ & & 202399 & 84333 & 286732 & 945945 & 1232010 & Jet Transfer & 77.7 \\
\hline \multicolumn{2}{|l|}{ net hours } & $16.4 \%$ & $6.8 \%$ & $23.3 \%$ & $76.8 \%$ & $100.0 \%$ & Sampl\&Prep. & 240.0 \\
\hline & & 0.0 & 0.0 & 77.7 & transfer & batch total & (days) & 13.2 \\
\hline
\end{tabular}

\begin{tabular}{|c|c|c|c|c|c|c|c|c|}
\hline Batch $\# 1$ & $\begin{array}{c}\text { starting } \\
\text { level }\end{array}$ & water & salt & total & crystallized & total & Inhib'Water & 210.6 \\
\hline depth (in) & & & dissolved & supernate & salt cake & content & Dissolution & 131.8 \\
\hline gallons & 271.50 & 40.0 & 16.67 & 58.67 & 254.83 & 313.5 & ExtraMixing & 168.0 \\
\hline$\frac{1}{\%}$ & 952965 & 140400 & 58500 & 205920 & 894465 & 1100385 & Jet Transfer & 55.3 \\
\hline \multirow{2}{*}{\multicolumn{2}{|c|}{ net hours }} & $12.8 \%$ & $5.3 \%$ & $18.7 \%$ & $81.3 \%$ & $100.0 \%$ & Sampl\&Prep. & 240.0 \\
\hline & & 210.6 & 131.8 & 55.3 & transfer & batch total & 1 (days) & 33.6 \\
\hline
\end{tabular}

\begin{tabular}{|c|c|c|c|c|c|c|c|c|}
\hline \multirow{2}{*}{ Batch 12} & \multirow[b]{2}{*}{$\begin{array}{c}\text { starting } \\
\text { level }\end{array}$} & \multirow{3}{*}{$\begin{array}{l}\text { water } \\
\text { added }\end{array}$} & \multirow{3}{*}{$\begin{array}{c}\text { salt } \\
\text { dissolved }\end{array}$} & \multirow{3}{*}{$\begin{array}{c}\text { total } \\
\text { supernate }\end{array}$} & \multicolumn{3}{|c|}{ 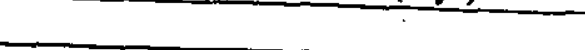 } & \multirow{2}{*}{210.6} \\
\hline & & & & & crystallized & total & Inhib'Water & \\
\hline depth (in) & 256.83 & & & & salt cake & content & Dissolution & 131.8 \\
\hline gallons & 901485 & 40.0 & 16.67 & 58.67 & 240.17 & 298.8 & ExtraMixing & 168.0 \\
\hline$\%$ & $\frac{901485}{0500}$ & 140400 & 58500 & 205920 & 842985 & 1048905 & Jet Transfer & 55.3 \\
\hline \multirow{2}{*}{\multicolumn{2}{|c|}{ net hours }} & $13.4 \%$ & $5.6 \%$ & $19.6 \%$ & $80.4 \%$ & $100.0 \%$ & Sampl\&Prep. & 240.0 \\
\hline & & 210.6 & 131.8 & 55.3 & transfer & batch total & (days) & 33.6 \\
\hline
\end{tabular}

\begin{tabular}{|c|c|c|c|c|c|c|c|c|}
\hline Batch $\# 3$ & & & & & & & & \\
\hline & $\begin{array}{c}\text { starting } \\
\text { level }\end{array}$ & water & salt & total & crystallized & total & Inhib'Water & $\overline{210.6}$ \\
\hline depth (in) & 242.17 & & dissolved & supernate & salt cake & content & Dissolution & 131.8 \\
\hline gallons & 850005 & 40.0 & 16.67 & 58.67 & 225.50 & 284.2 & ExtraMixing & 168.0 \\
\hline $\mathscr{\%}$ & 8529 & 140400 & 58500 & 205920 & 791505 & 997425 & Jet Transfer & 55.3 \\
\hline net hours & $85.2 \%$ & $14.1 \%$ & $5.9 \%$ & $20.6 \%$ & $79.4 \%$ & $100.0 \%$ & Sampl\&Prep. & 240.0 \\
\hline & & 210.6 & 131.8 & 55.3 & transfer & batch total & (days) & 33.6 \\
\hline
\end{tabular}

$7.4 \mathrm{gpm}$

$60 \mathrm{gpm}$

2 Flygt Mixers with 50C Heated IW: 


\begin{tabular}{|c|c|c|c|c|c|c|c|c|}
\hline Batch \#4 & & & & & & & Step & Hours \\
\hline & $\begin{array}{l}\text { level } \\
\text { lestiog }\end{array}$ & $\begin{array}{l}\text { water } \\
\text { added }\end{array}$ & salt & total & crystallized & to & Inhib'Water & 210.6 \\
\hline depth (in) & 227.50 & & dissolved & superate & salt cake & content & Dissolution & 131.8 \\
\hline gallons & 798525 & $\begin{array}{r}40.0 \\
\end{array}$ & 16.67 & 58.67 & 210.83 & 269.5 & ExtraMixing & 168.0 \\
\hline$\%$ & $\frac{198520}{8409}$ & 140400 & 58500 & 205920 & 740025 & 945945 & Jet Transfer & 55.3 \\
\hline \multirow{2}{*}{\multicolumn{2}{|c|}{ net hours }} & $14.8 \%$ & $6.2 \%$ & $21.8 \%$ & $78.2 \%$ & $100.0 \%$ & Sampl\&Prep. & 240.0 \\
\hline & & 210.6 & 131.8 & 55.3 & transfer & batch total & (days) & 33.6 \\
\hline
\end{tabular}

\section{(Case 13) Three Flygt Mixers with 50C Heated Inhibited Water}

Tank Level at the End of Batch $4=$

Salt Cake Level Reduced $=$

Total Inhibited Water Used=

dissolution with mixer (a) $=$

extra mixing (b)

total mixer running time $(a+b)=$

inhibited water addition $=$

jet transfer time $=$.

test sample \& preparation $=$

total time $=$

$$
210.8+2=
$$

$$
269.5-210.8=
$$

561600 gallons =

527.0 hrs $=$

$672.0 \mathrm{hrs}$

$842.4 \mathrm{hrs}=$

$298.7 \mathrm{hrs}=$

$1200.0 \mathrm{hrs}=$

$3540.1 \mathrm{hrs}=$
212.8 "TK Lvl

58.7 "TK Lvl

160.0 " TK Lvl

22.0 days

28.0 days

50.0 days

35.1 days

12.4 days

50.0 days

or 147.5 days

After the existing supernate transfer to TK 30 and the above four batch transfer to TK 26,

Tank 37 salt level of 212.8 inch will pass the project scope tank level of 227 inches.

Batch $\# 5$ is shown below even though it is not required to meet the original project scope level of 227 inches. The Tank 37 level after Batch 5 will be

\begin{tabular}{|c|c|c|c|c|c|c|c|c|}
\hline Batch \#5 & & & & & & & Step & Hours \\
\hline & level & $\begin{array}{l}\text { water } \\
\text { added }\end{array}$ & $\begin{array}{c}\text { salt } \\
\text { dissolved }\end{array}$ & total & crystallized & & Inhib'Water & 210.6 \\
\hline depth (in) & 212.83 & 400 & & supernate & salt cake & content & Dissolution & 131.8 \\
\hline gallons & 747045 & 40.0 & 16.67 & 58.67 & 196.17 & 254.8 & ExtraMixing & 168.0 \\
\hline$\%$ & $83.5 \%$ & 140400 & 58500 & 205920 & 688545 & 894465 & Jet Transfer & 55.3 \\
\hline \multirow{2}{*}{\multicolumn{2}{|c|}{ net hours }} & $15.7 \%$ & $6.5 \%$ & 23.0\% & $77.0 \%$ & $100.0 \%$ & Sampl\&Prep. & 240.0 \\
\hline & & 210.6 & 131.8 & 55.3 & transfer & batch total & (days) & 33.6 \\
\hline
\end{tabular}

198.2 inches if Batch 5 is processed. 


\section{TANK 37H DISSOLUTION CYCLE ANALYSIS}

\begin{tabular}{|c|c|c|c|c|}
\hline $\begin{array}{l}\text { ase 14) Two Flygt Mixers with } 75 \mathrm{C} \mathrm{He} \\
\text { Water to salt dissolution ratio }=\end{array}$ & 2.4 & to & 1 & $\%$ total time \\
\hline 2 Flygt Mixers & total & & & 100 \\
\hline 8.6 gpm dissolution rate; dissolut & 46.9 & days & & $32.47 \%$ \\
\hline
\end{tabular}

a) initial salt cake level (average of $262^{\prime \prime} \& \cdot 277^{\prime \prime}$ ) = 269.50 inch

b) inhibited water batch $=\sim 80 \%$ of $10 \mathrm{Kgal}$ tank $=$ inhibited water pumping to TK 37 (80 to $120 \mathrm{gpm})=$ 8000 gallons net pumping time of one batch IW to TK $37=$ IWp $=$ number of IW batches per $12 \mathrm{hr}$-shift = number of IW batches per day $=$ total time of 1 batch IW addition to TK $37=$ IWt $=$ total IW time needed/net pumping time $=$ IWU/Wp $=$

$100 \mathrm{gpm}$

$1.3 \mathrm{hrs}$

1 batch per shift

2 batches per day

$12 \mathrm{brs}$

9

c) salt dissolution rate $=$

$8.6 \mathrm{gpm}$

d) jet transfer rate from TK 37 to TK 30 or TK $26=$ (60 to $100 \mathrm{gpm}$ )

$60 \mathrm{gpm}$

2 Flygt Mixers with $75 \mathrm{C}$ Heated IW:

\begin{tabular}{|c|c|c|c|c|c|c|c|c|}
\hline \multicolumn{7}{|c|}{2 Flygt Mixers with $75 \mathrm{C}$ Heated IW: } & \multicolumn{2}{|l|}{ Step } \\
\hline Decant & salt cake & water & salt & total liq or & crystallized & total & Inhib'Water & 0.0 \\
\hline Rxisting & I dissolv. & existing & dissolved & supernate & salt cake & content & Dissolution & 0.0 \\
\hline depth (in) & 293.53 & 57.66 & 24.03 & 81.69 & 269.50 & 351.0 & ExtraMixing & 0.0 \\
\hline gallons & 1030278 & 202399 & 84333 & 286732 & 945945 & 1232010 & Jet Transfer & 77.7 \\
\hline$\%$ & $83.6 \%$ & $16.4 \%$ & $6.8 \%$ & $23.3 \%$ & $76.8 \%$ & $100.0 \%$ & Sampl\&Prep. & 240.0 \\
\hline net hours & & 0.0 & 0.0 & $\overline{77.7}$ & transfer & batch tota & (days) & 13.2 \\
\hline
\end{tabular}

\begin{tabular}{|c|c|c|c|c|c|c|c|c|}
\hline Batch \#1 & $\begin{array}{c}\text { starting } \\
\text { level }\end{array}$ & $\begin{array}{l}\text { water } \\
\text { added }\end{array}$ & $\begin{array}{c}\text { salt } \\
\text { dissolved }\end{array}$ & $\begin{array}{c}\text { total } \\
\text { supernate }\end{array}$ & $\begin{array}{l}\text { crystallized } \\
\text { salt cake }\end{array}$ & $\begin{array}{c}\text { total } \\
\text { content }\end{array}$ & $\begin{array}{l}\text { Inhib'Water } \\
\text { Dissolution }\end{array}$ & $\begin{array}{l}210.6 \\
113.4\end{array}$ \\
\hline depth (in) & 271.50 & 40.0 & 16.67 & 58.67 & 254.83 & 313.5 & ExtraMixing & 168.0 \\
\hline gallons & 952965 & 140400 & 58500 & 205920 & 894465 & 1100385 & Jet & 55.3 \\
\hline$\%$ & $86.6 \%$ & $12.8 \%$ & $5.3 \%$ & $18.7 \%$ & $81.3 \%$ & $100.0 \%$ & Sampl\& & 240.0 \\
\hline net hours & & 210.6 & 113.4 & 55.3 & transfer & batch total & 1 (days) & 32.8 \\
\hline
\end{tabular}

\begin{tabular}{|c|c|c|c|c|c|c|c|c|}
\hline Batch H2 & $\begin{array}{c}\text { starting } \\
\text { level }\end{array}$ & $\begin{array}{l}\text { water } \\
\text { added }\end{array}$ & $\begin{array}{c}\text { salt } \\
\text { dissolved }\end{array}$ & $\begin{array}{c}\text { total } \\
\text { supernate }\end{array}$ & $\begin{array}{l}\text { crystallized } \\
\text { salt cake }\end{array}$ & $\begin{array}{c}\text { total } \\
\text { content }\end{array}$ & $\begin{array}{l}\text { Inhib'Water } \\
\text { Dissolution }\end{array}$ & $\begin{array}{l}210.6 \\
113.4\end{array}$ \\
\hline depth (in) & 256.83 & 40.0 & 16.67 & 58.67 & 240.17 & 298.8 & ExtraMixing & 168.0 \\
\hline gallons & 901485 & 140400 & 58500 & 205920 & 842985 & 1048905 & Jet Transfer & 55.3 \\
\hline$\%$ & $85.9 \%$ & $13.4 \%$ & $5.6 \%$ & $19.6 \%$ & $80.4 \%$ & $100.0 \%$ & Sampl\&Prep. & 240.0 \\
\hline net hours & & 210.6 & 113.4 & \multicolumn{2}{|c|}{ 55.3 transfer } & batch total & 1 (days) & 32.8 \\
\hline
\end{tabular}

\begin{tabular}{|c|c|c|c|c|c|c|c|c|}
\hline Batch \#3 & $\begin{array}{c}\text { starting } \\
\text { level }\end{array}$ & $\begin{array}{l}\text { water } \\
\text { added }\end{array}$ & $\begin{array}{c}\text { salt } \\
\text { dissolved }\end{array}$ & $\begin{array}{c}\text { total } \\
\text { supernate }\end{array}$ & $\begin{array}{c}\text { crystallized } \\
\text { salt cake }\end{array}$ & $\begin{array}{c}\text { total } \\
\text { content }\end{array}$ & $\begin{array}{l}\text { Inhib'Water } \\
\text { Dissolution }\end{array}$ & $\begin{array}{l}210.6 \\
113.4\end{array}$ \\
\hline depth (in) & 242.17 & 40.0 & 16.67 & 58.67 & 225.50 & 284.2 & ExtraMixing & 168.0 \\
\hline gallons & 850005 & 140400 & 58500 & 205920 & 791505 & 997425 & Jet Transfer & 55.3 \\
\hline$\%$ & $85.2 \%$ & $14.1 \%$ & $5.9 \%$ & $20.6 \%$ & $79.4 \%$ & $100.0 \%$ & Sampl\&Prep. & 240.0 \\
\hline net hours & & 210.6 & 113.4 & 55.3 & transfer & batch tota & 1 (days) & 32.8 \\
\hline
\end{tabular}




\begin{tabular}{|c|c|c|c|c|c|c|c|c|}
\hline & & & & & & & Step & Hours \\
\hline$\frac{\text { Batch \#4 }}{\text { 棌 }}$ & $\begin{array}{l}\text { starting } \\
\text { level }\end{array}$ & $\begin{array}{l}\text { water } \\
\text { added }\end{array}$ & $\begin{array}{c}\text { salt } \\
\text { dissolved }\end{array}$ & total & $\begin{array}{l}\text { crystallized } \\
\text { salt cake }\end{array}$ & $\begin{array}{c}\text { total } \\
\text { content }\end{array}$ & $\begin{array}{l}\text { Inhib'Water } \\
\text { Dissolution }\end{array}$ & 210.6 \\
\hline depth (in) & 227.50 & 40.0 & 16.67 & 58.67 & 210.83 & 269.5 & ExtraMixing & 168.0 \\
\hline gallons & 798525 & 140400 & 58500 & 205920 & 740025 & 945945 & Jet Transfer & 55.3 \\
\hline$\%$ & $84.4 \%$ & $14.8 \%$ & $6.2 \%$ & $21.8 \%$ & $78.2 \%$ & $100.0 \%$ & Sampl\&Prep. & 240.0 \\
\hline net hours & & 210.6 & 113.4 & 55.3 & transfer & batch total & 1 (days) & 32.8 \\
\hline
\end{tabular}

\section{(Case 14) Two Flygt Mixers with 75C Heated Inhibited Water}

Tank Level at the End of Batch $4=$

Salt Cake Level Reduced $=$

Total Inhibited Water Used=

dissolution with mixer (a) $=$

extra mixing (b)

total mixer running time $(a+b)=$

inhibited water addition $=$

jet transfer time $=$

test sample \& preparation=

total time $=$
$210.8+2=$

$269.5-210.8=$

561600 gallons =

$453.5 \mathrm{hrs}=$

$672.0 \mathrm{hrs}$

842.4 hrs $=$

298.7 hrs $=$ $1200.0 \mathrm{hrs}=$

$3466.6 \mathrm{brs}=$
$212.8 * \mathrm{TK} \mathrm{Lvl}$

$58.7 *$ TK Lvl

160.0 * TK LvI

18.9 days

28.0 days

46.9 days

35.1 days

12.4 days

50.0 days

144.4 days

4.7 months

After the existing supernate transfer to TK 30 and the above four batch transfer to TK 26, Tank 37 salt level of 212.8 inch will pass the project scope tank level of 227 inches.

Batch $\# 5$ is shown below cven though it is not required to meet the original project scope level of 227 inches. The Tank 37 level after Batch 5 will be

198.2 inches if Batch 5 is processed. .

\begin{tabular}{|c|c|c|c|c|c|c|c|c|}
\hline \multirow{3}{*}{ Batch \#S } & & & & & & & Step & Hours \\
\hline & starting & water & salt & total & crystallized & tots & Inhib'Water & 210.6 \\
\hline & level & added & dissolved & supernate & salt cake & content & Dissolution & 113.4 \\
\hline depth (in) & 212.83 & 40.0 & 16.67 & 58.67 & 196.17 & 254.8 & ExtraMixing & 168.0 \\
\hline gallons & 747045 & 140400 & 58500 & 205920 & 688545 & 894465 & Jet Transfer & 55.3 \\
\hline$\%$ & $83.5 \%$ & $15.7 \%$ & $6.5 \%$ & $23.0 \%$ & $77.0 \%$ & $100.0 \%$ & Sampl\&Prep. & 240.0 \\
\hline \multicolumn{2}{|l|}{ net hours } & 210.6 & 113.4 & 55.3 & transfer & batch tota & 1 (days) & 32.8 \\
\hline
\end{tabular}




\section{TANK 37H DISSOLUTION CYCLE ANALYSIS}

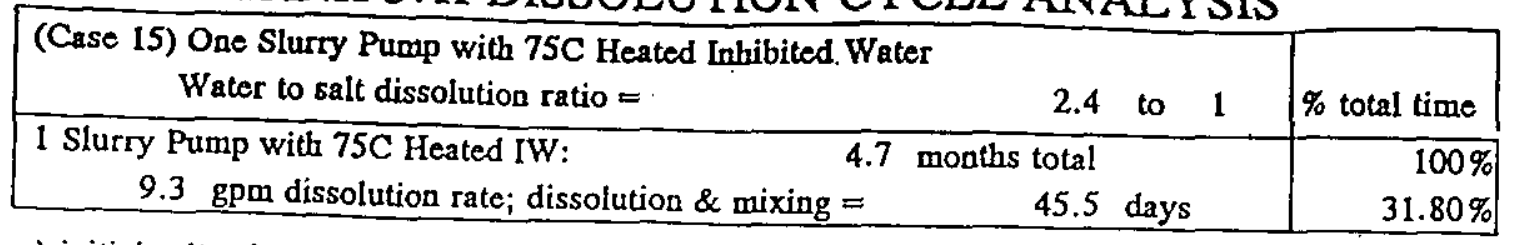

a) initial salt cake level (average of $262^{\prime \prime} \& 277^{\prime \prime}$ ) =

b) inhibited water batch $=\sim 80 \%$ of $10 \mathrm{Kgal}$ tank $=$ inhibited water pumping to TK 37 (80 to $120 \mathrm{gpm})=$ net pumping time of one batch IW to TK $37=$ IWp $=$ number of IW batches per $12 \mathrm{hr}$-shift = number of IW batches per day = total time of 1 batch IW addition to TK $37=$ IWt $=$ total IW time needed/net pumping time $=I \mathrm{~W} / \mathrm{IW} \mathrm{p}=$ 269.50 inch c) salt dissolution rate $=$

d) jet transfer rate from TK 37 to TK 30 or TK $26=$ (60 to $100 \mathrm{gpm}$ )

$\begin{aligned} 8000 & \text { gallons } \\ 100 & \text { gpm } \\ 1.3 & \text { hrs } \\ 1 & \text { batch per shift } \\ 2 & \text { batches per day } \\ 12 & \text { hrs } \\ 9 & \end{aligned}$

$9.3 \mathrm{gpm}$

$60 \mathrm{gpm}$

1 Slurry Pump with 75C Heated IW:

\begin{tabular}{|c|c|c|c|c|c|c|c|c|}
\hline $\mathrm{De}$ & & & & & & & Step & Hours \\
\hline & salt ce & water & salt & total liq or & crysta & tatal & Inhib' Water & 0.0 \\
\hline & t aissolv. & & dissolved & supernate & salt cake & content & Dissolution & 0.0 \\
\hline depth (in) & 293.53 & 57.66 & 24.03 & 81.69 & 269.50 & 351.0 & ExtraMixing & 0.0 \\
\hline gallons & 1030278 & 202399 & 84333 & 286732 & 945945 & 1232010 & Jet Transfer & 77.7 \\
\hline$\%$ & $83.6 \%$ & $16.4 \%$ & $6.8 \%$ & $23.3 \%$ & $76.8 \%$ & $100.0 \%$ & Sampl\&Prep. & 240.0 \\
\hline net hours & & 0.0 & 0.0 & 77.7 & transfer & batch total & (days) & 13.2 \\
\hline
\end{tabular}

\begin{tabular}{|c|c|c|c|c|c|c|c|c|}
\hline Batch \#1 & $\begin{array}{c}\text { starting } \\
\text { level }\end{array}$ & $\begin{array}{l}\text { water } \\
\text { added }\end{array}$ & $\begin{array}{c}\text { salt } \\
\text { dissolved }\end{array}$ & $\begin{array}{c}\text { total } \\
\text { supernate }\end{array}$ & $\begin{array}{l}\text { crystallized } \\
\text { salt cake }\end{array}$ & $\begin{array}{c}\text { total } \\
\text { content }\end{array}$ & $\begin{array}{l}\text { Inhib'Water } \\
\text { Dissolution }\end{array}$ & $\begin{array}{l}210.6 \\
104.8\end{array}$ \\
\hline depth (in) & 271.50 & 40.0 & 16.67 & 58.67 & 254.83 & 313.5 & ExtraMixing & 168.0 \\
\hline gallons & 952965 & 140400 & 58500 & 205920 & 894465 & 1100385 & Jet Transfer & 55.3 \\
\hline$\%$ & $86.6 \%$ & $12.8 \%$ & $5.3 \%$ & $18.7 \%$ & $81.3 \%$ & $100.0 \%$ & Sampl\&Prep. & 240.0 \\
\hline net hours & & 210.6 & 104.8 & 55.3 & transfer & batch total & (days) & $32 . \overline{4}$ \\
\hline
\end{tabular}

\begin{tabular}{|c|c|c|c|c|c|c|c|c|}
\hline Batch \#2 & $\begin{array}{c}\text { starting } \\
\text { level }\end{array}$ & $\begin{array}{l}\text { water } \\
\text { added }\end{array}$ & $\begin{array}{c}\text { salt } \\
\text { dissolved }\end{array}$ & $\begin{array}{c}\text { total } \\
\text { supernate }\end{array}$ & $\begin{array}{c}\text { crystallized } \\
\text { salt cake }\end{array}$ & $\begin{array}{c}\text { total } \\
\text { content }\end{array}$ & $\begin{array}{l}\text { Inhib'Water } \\
\text { Dissolution }\end{array}$ & $\begin{array}{l}210.6 \\
104.8\end{array}$ \\
\hline depth (in) & 256.83 & 40.0 & 16.67 & 58.67 & 240.17 & 298.8 & Ex & 168.0 \\
\hline gallons & 901485 & 140400 & 58500 & 205920 & 842985 & 1048905 & Jet & 55.3 \\
\hline$\%$ & $85.9 \%$ & $.4 \%$ & $5.6 \%$ & $19.6 \%$ & $80.4 \%$ & $100.0 \%$ & Samp & 240.0 \\
\hline net hours & & 210.6 & 104.8 & 55.3 & transfer & batch total & (days) & 32.4 \\
\hline
\end{tabular}

\begin{tabular}{|c|c|c|c|c|c|c|c|c|}
\hline Batch $\# 3$ & $\begin{array}{c}\text { starting } \\
\text { level }\end{array}$ & $\begin{array}{l}\text { water } \\
\text { added }\end{array}$ & $\begin{array}{c}\text { salt } \\
\text { dissolved }\end{array}$ & $\begin{array}{c}\text { total } \\
\text { supernate }\end{array}$ & $\begin{array}{l}\text { crystallized } \\
\text { salt cake }\end{array}$ & $\begin{array}{c}\text { total } \\
\text { content }\end{array}$ & $\begin{array}{l}\text { Inhib'Water } \\
\text { Dissolution }\end{array}$ & $\begin{array}{l}210.6 \\
104.8\end{array}$ \\
\hline depth (in) & 242.17 & 40.0 & 16.67 & 58.67 & 225.50 & 284.2 & ExtraMixing & 168.0 \\
\hline gallons & 850005 & 140400 & 58500 & 205920 & 791505 & 997425 & Jet Transfer & 55.3 \\
\hline$\%$ & $85.2 \%$ & $14.1 \%$ & $5.9 \%$ & $20.6 \%$ & $79.4 \%$ & $100.0 \%$ & Sampl\&\&rep. & 240.0 \\
\hline net hours & & 210.6 & 104.8 & 55.3 & transfer & batch tota & I (days) & 32.4 \\
\hline
\end{tabular}




\begin{tabular}{|c|c|c|c|c|c|c|c|c|}
\hline Batch & & & & & & & Step & Hours \\
\hline & $\begin{array}{c}\text { scarting } \\
\text { level }\end{array}$ & $\begin{array}{l}\text { water } \\
\text { added }\end{array}$ & $\begin{array}{c}\text { salt } \\
\text { dissolved }\end{array}$ & total & crystallized & total & Inhib'Water & 210.6 \\
\hline depth (in) & 227.50 & 40.0 & 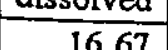 & & & content & Dissolution & 104.8 \\
\hline gallons & 798525 & 140400 & 16.67 & 58.67 & 210.83 & 269.5 & ExtraMixing & 168.0 \\
\hline$\%$ & 84.49 & 140400 & 58500 & 205920 & 740025 & 945945 & Jet Transfer & 55.3 \\
\hline \multicolumn{2}{|l|}{ net hours } & $14.8 \%$ & $6.2 \%$ & $21.8 \%$ & $78.2 \%$ & $100.0 \%$ & Sampl\&Prep. & 240.0 \\
\hline & & 210.6 & 104.8 & 55.3 & transfer & batch total & 1 (days) & 32.4 \\
\hline
\end{tabular}

\section{(Case 15) One Slurry Pump with 75C Heated Inhibited Water}

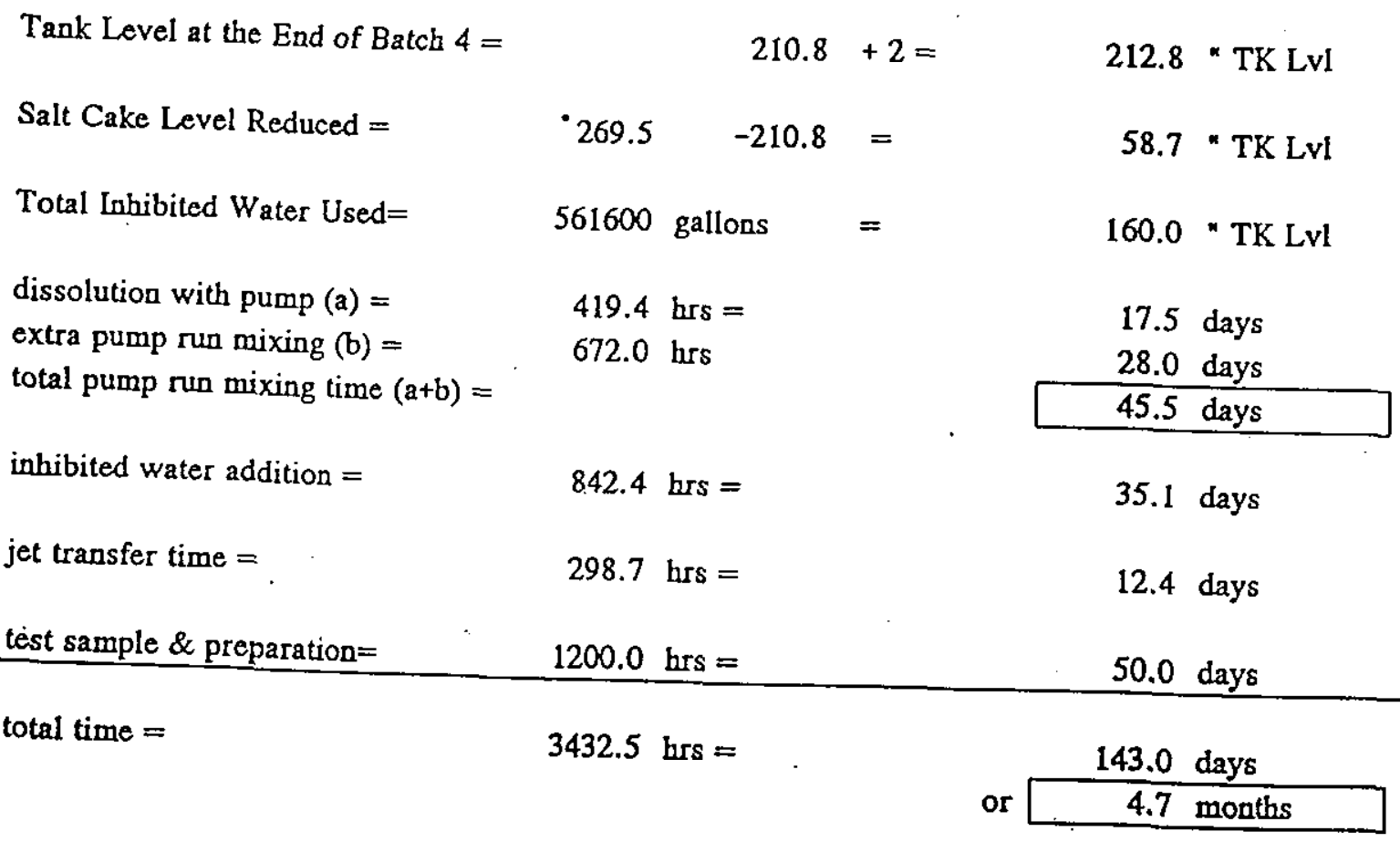

After the existing supernate transfer to TK 30 and the above four batch transfer to TK 26 ,
Tank 37 salt level of 212.8 inch will pass the project scopo 212.8 inch will pass the project scope tank level of 227 inches.

Batch $\# 5$ is shown below even though it is not required to meet the original project scope level of 227 inches. The Tank 37 level after Batch 5 will be

198.2 inches if Batch 5 is processed.

\begin{tabular}{|c|c|c|c|c|c|c|c|c|}
\hline Batch $\# 5$ & & & & & & & Step & Hours \\
\hline & level & $\begin{array}{l}\text { Water } \\
\text { added }\end{array}$ & salt & total & crystallized & & Inhib'Water & $\overline{210.6}$ \\
\hline depth (in) & 212.83 & & & superviate & salt cake & content & Dissolution & 104.8 \\
\hline gallons & 747045 & 40.0 & 16.67 & 58.67 & 196.17 & 254.8 & ExtraMixing & 168.0 \\
\hline$\%$ & $\frac{147045}{83.5 \%}$ & 140400 & 58500 & 205920 & 688545 & 894465 & Jet Transfer & 55.3 \\
\hline net hours & $83.5 \%$ & $15.7 \%$ & $6.5 \%$ & $23.0 \%$ & $77.0 \%$ & $100.0 \%$ & Sampl\&Prep. & 240.0 \\
\hline & & 210.6 & 104.8 & 55.3 & transfer & batch total & I (days) & 32.4 \\
\hline
\end{tabular}




\section{TANK 37H DISSOLUTION CYCLE ANALYSIS}

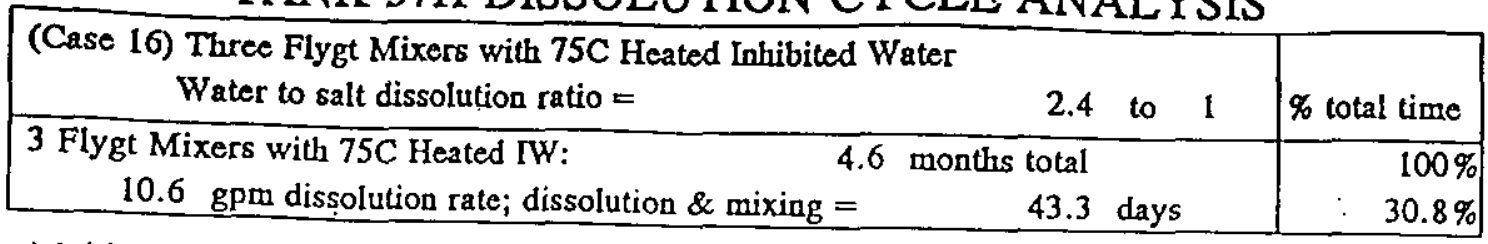

a) initial salt cake level (average of $262^{\prime \prime} \& 277^{\prime \prime}$ ) =

b) inhibited water batch $=\sim 80 \%$ of $10 \mathrm{Kgal}$ tank $=$ inhibited water pumping to TK 37 (80 to $120 \mathrm{gpm}$ ) = net pumping time of one batch IW to TK $37=\mathrm{IW}$ = number of IW batches per $12 \mathrm{hr}$-shift $=$ number of IW batches per day = total time of 1 batch IW addition to TK $37=$ IWt $=$ total IW time needed/net pumping time $=I W t / I W p=$ 269.50 inch 8000 gallons $100 \mathrm{gpm}$

$1.3 \mathrm{hrs}$

1 batch per shift

2 batches per day

$12 \mathrm{hrs}$

9

c) salt dissolution rate $=$

$10.6 \mathrm{gpm}$

d) jet transfer rate from TK 37 to TK 30 or TK $26=$ (60 to $100 \mathrm{gpm}$ )

$60 \mathrm{gpm}$

3 Flygt Mixers with 75C Heated IW:

\begin{tabular}{|c|c|c|c|c|c|c|c|c|}
\hline \multicolumn{9}{|c|}{3 Flygt Mixers with 75C Heated IW: } \\
\hline Decant & salt cake & water & salt & total liq or & crystallized & total & Inhib'Water & 0.0 \\
\hline Existing & + dissolv. & existing & dissolved & supernate & salt cake & content & Dissolution & 0.0 \\
\hline depth (in) & 293.53 & 57.66 & 24.03 & 81.69 & 269.50 & 351.0 & ExtraMixing & 0.0 \\
\hline gallons & 1030278 & 202399 & 84333 & 286732 & 945945 & 1232010 & Jet Transfer & 77.7 \\
\hline$\%$ & $83.6 \%$ & $16.4 \%$ & $6.8 \%$ & $23.3 \%$ & $76.8 \%$ & $100.0 \%$ & Sampl\&Prep. & 240.0 \\
\hline net hours & & 0.0 & 0.0 & 77.7 & transfer & batch tota & (days) & 13.2 \\
\hline
\end{tabular}

\begin{tabular}{|c|c|c|c|c|c|c|c|c|}
\hline Batch \#1 & $\begin{array}{c}\text { starting } \\
\text { level }\end{array}$ & $\begin{array}{l}\text { water } \\
\text { added }\end{array}$ & $\begin{array}{c}\text { salt } \\
\text { dissolved }\end{array}$ & total & crystallized & total & Inhib'Water & 210.6 \\
\hline depth (in) & 271.50 & 40.0 & 16.67 & & & & & 92.0 \\
\hline gallons & 952965 & 40400 & $\frac{10.01}{58500}$ & 67 & 254.83 & 313.5 & Extr & 168.0 \\
\hline$\%$ & $86.6 . \%$ & & sosw & .05920 & 894465 & 1100385 & Jet $\mathrm{T}$ & 55.3 \\
\hline \multirow{2}{*}{\multicolumn{2}{|c|}{ net hours }} & $\frac{12.0 \times 0}{2106}$ & $5.3 \%$ & $18.7 \%$ & $81.3 \%$ & $100.0 \%$ & Sampl\& & 240.0 \\
\hline & & 210.6 & 92. & 5.3 & sfer & batch total & (days) & 31.9 \\
\hline
\end{tabular}

\begin{tabular}{|c|c|c|c|c|c|c|c|c|}
\hline Batch $\# 2$ & $\begin{array}{c}\text { starting } \\
\text { level }\end{array}$ & $\begin{array}{l}\text { water } \\
\text { added }\end{array}$ & $\begin{array}{c}\text { salt } \\
\text { dissolved }\end{array}$ & $\begin{array}{c}\text { total } \\
\text { supernate }\end{array}$ & $\begin{array}{c}\text { crystallized } \\
\text { salt cake }\end{array}$ & $\begin{array}{c}\text { total } \\
\text { content }\end{array}$ & $\begin{array}{l}\text { Inhib'Water } \\
\text { Dissolution }\end{array}$ & $\begin{array}{r}210.6 \\
92.0\end{array}$ \\
\hline depth (in) & 256.83 & 40.0 & 16.67 & 58.67 & 240.17 & 298.8 & Ext & 168.0 \\
\hline gallons & 901485 & 140400 & 58500 & 205920 & 842985 & 1048905 & Jet & 55.3 \\
\hline$\%$ & $85.9 \%$ & $13.4 \%$ & $5.6 \%$ & $19.6 \%$ & $80.4 \%$ & $100.0 \%$ & Sampl\&Prep. & 240.0 \\
\hline get hours & & 210.6 & 92.0 & 55.3 & transfer & batch total & 1 (days) & 31.9 \\
\hline
\end{tabular}

\begin{tabular}{|c|c|c|c|c|c|c|c|c|}
\hline Batch $\neq 3$ & $\begin{array}{c}\text { starting } \\
\text { level }\end{array}$ & $\begin{array}{l}\text { water } \\
\text { added }\end{array}$ & $\begin{array}{c}\text { salt } \\
\text { dissolved }\end{array}$ & total & crystallized & total & Inhib'Water & 210.6 \\
\hline depth (in) & 242.17 & 40.0 & 16.67 & 58.67 & 225.50 & $\frac{\text { content }}{2842}$ & Diss & $\begin{array}{r}92.0 \\
168.0\end{array}$ \\
\hline gallons & 850005 & 140400 & 58500 & 205920 & 791505 & $\frac{284.2}{997425}$ & ExtraMixing & $\begin{array}{r}108.0 \\
55.3\end{array}$ \\
\hline $\mathscr{\%}$ & $85.2 \%$ & $14.1 \%$ & $5.9 \%$ & $20.6 \%$ & $79.4 \%$ & $100.0 \%$ & Sampl\&Prep. & 240.0 \\
\hline net hours & & 210.6 & 92.0 & 55.3 & transfer & batch tota & (days) & 31.9 \\
\hline
\end{tabular}




\begin{tabular}{|c|c|c|c|c|c|c|c|c|}
\hline & & & & & & & Step & Hours \\
\hline Batch \#4 & $\begin{array}{l}\text { starting } \\
\text { level }\end{array}$ & $\begin{array}{l}\text { water } \\
\text { added }\end{array}$ & $\begin{array}{c}\text { salt } \\
\text { dissolved }\end{array}$ & $\begin{array}{c}\text { total } \\
\text { supernate }\end{array}$ & $\begin{array}{l}\text { crystallized } \\
\text { salt cake }\end{array}$ & $\begin{array}{c}\text { total } \\
\text { content }\end{array}$ & $\begin{array}{l}\text { Inhib'Water } \\
\text { Dissolution }\end{array}$ & $\begin{array}{r}210.6 \\
92.0\end{array}$ \\
\hline depth (in) & 227.50 & 40.0 & 16.67 & 58.67 & 210.83 & 269.5 & ExtraMixing & 168.0 \\
\hline gallons & 798525 & 140400 & 58500 & 205920 & 740025 & 945945 & Jet Transfer & 55.3 \\
\hline$\%$ & $84.4 \%$ & $14.8 \%$ & $6.2 \%$ & $21.8 \%$ & $78.2 \%$ & $100.0 \%$ & Sampl\&Prep. & 240.0 \\
\hline net hours & & 210.6 & 92.0 & 55.3 & transfer & batch total & 1 (days) & 31.9 \\
\hline
\end{tabular}

\section{(Case 16) Three Flygt Mixers with 75C Heated Inhibited Water}

Tank Level at the End of Batch $4=$

Salt Cake Level Reduced $=$

Total Inhibited Water Used=

dissolution with mixer (a) $=$

extra mixing (b)

total mixer running time $(a+b)=$

inhibited water addition $=$

jet transfer time $=$

test sample \& preparation=

total time $=$

$$
210.8+2=
$$$$
269.5-210.8=
$$

561600 gallons $=$

$367.9 \mathrm{hrs}=$

$672.0 \mathrm{hrs}$

$842.4 \mathrm{hrs}=$

$298.7 \mathrm{hrs}=$

$1200.0 \mathrm{hrs}=$

$3381.0 \mathrm{hrs}=$
212.8 "TK LvI

58.7 "TK Lvl

160.0 * TK LvI

15.3 days

28.0 days

43.3 days

35.1 days

12.4 days

50.0 days

140.9 days

or 4.6 months

After the existing supernate transfer to TK 30 and the above four batch transfer to TK 26 ,

Tank 37 salt level of 212.8 inch will pass the project scope tank level of 227 inches.

Batch \#5 is shown below even though it is not required to meet the original project scope level of 227 inches. The Tank 37 level after Batch 5 will be

198.2 inches if Batch 5 is processed.

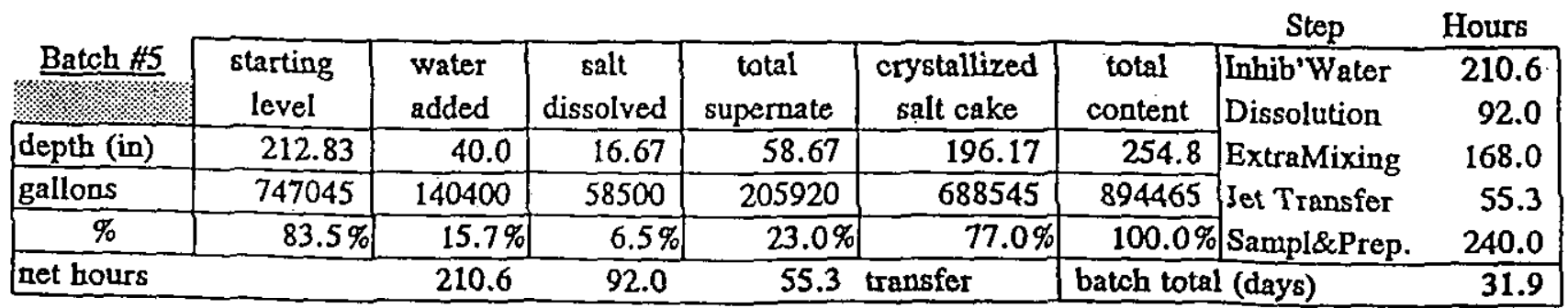


Appendix 3

Heat Transfer Calculations

\begin{tabular}{|c|c|c|c|c|c|}
\hline Method of Dissolution & $\begin{array}{c}\text { Water } \\
\text { Temp. }\left({ }^{\circ} \mathrm{C}\right)\end{array}$ & $\begin{array}{c}\text { End IW Addition } \\
\text { Batch } 1 \\
\text { Batch } 4 \\
\end{array}$ & $\begin{array}{c}\text { Peak Temp. } \\
\text { Batch 1 } \\
\text { Batch } 4 \\
\end{array}$ & $\begin{array}{c}\text { End Dissolution } \\
\text { Batch 1 } \\
\text { Batch } 4 \\
\end{array}$ & $\begin{array}{c}\text { End Mixing } \\
\text { Batch 1 } \\
\text { Batch } 4 \\
\end{array}$ \\
\hline 1. No Agitation & 25 & $\begin{array}{l}36.0 \\
29.7 \\
\end{array}$ & $\begin{array}{l}37.0 \\
29.9 \\
\end{array}$ & $\begin{array}{l}33.6 \\
27.6\end{array}$ & $\begin{array}{l}34.3 \\
27.9\end{array}$ \\
\hline 2. $\mathrm{MDG}$ & 25 & $\begin{array}{l}36.0 \\
29.1 \\
\end{array}$ & $\begin{array}{l}37.0 \\
29.1 \\
\end{array}$ & $\begin{array}{l}33.4 \\
26.5 \\
\end{array}$ & $\begin{array}{l}3 \overline{33.6} \\
27.0-\end{array}$ \\
\hline 3. $\mathrm{MDG}$ & 50 & $\begin{array}{l}38.0 \\
34.0 \\
\end{array}$ & $\begin{array}{l}38.0 \\
34.0 \\
\end{array}$ & $\begin{array}{l}35.0 \\
30.6 \\
\end{array}$ & $\begin{array}{l}35.0 \\
30.8 \\
\end{array}$ \\
\hline 4. $\mathrm{MDG}$ & 75 & $\begin{array}{l}40.1 \\
39.9 \\
\end{array}$ & $\begin{array}{l}40.1 \\
40.0 \\
\end{array}$ & $\begin{array}{l}37.3 \\
36.6 \\
\end{array}$ & $\begin{array}{l}37.0 \\
36.2 \\
\end{array}$ \\
\hline 5. One Flygt Mixer & $\overline{25}$ & $\begin{array}{l}36.0 \\
29.8 \\
\end{array}$ & $\begin{array}{l}37.0 \\
30.0 \\
\end{array}$ & $\begin{array}{l}35.6 \\
27.5 \\
\end{array}$ & $\begin{array}{l}35.9 \\
27.7 \\
\end{array}$ \\
\hline 6. Two Flygt Mixers & 25 & $\begin{array}{l}36.5 \\
30.0 \\
\end{array}$ & $\begin{array}{l}37.0 \\
30.2 \\
\end{array}$ & $\begin{array}{l}33.7 \\
27.6 \\
\end{array}$ & $\begin{array}{l}34.0 \\
28.2 \\
\end{array}$ \\
\hline 7. One Flygt Mixer & 50 & $\begin{array}{l}38.0 \\
35.4 \\
\end{array}$ & $\begin{array}{l}38.0 \\
35.4 \\
\end{array}$ & $\begin{array}{l}35.5 \\
32.5\end{array}$ & $\begin{array}{l}35.7 \\
32.7\end{array}$ \\
\hline 8. One Slurry Pump & 25 & $\begin{array}{l}36.0 \\
37.9 \\
\end{array}$ & $\begin{array}{l}38.6 \\
40.6 \\
\end{array}$ & $\begin{array}{l}36.1 \\
37.8 \\
\end{array}$ & $\begin{array}{l}38.6 \\
40.6 \\
\end{array}$ \\
\hline 9. Three Flygt Mixers & 25 & $\begin{array}{l}36.0 \\
30.4 \\
\end{array}$ & $\begin{array}{l}37.0 \\
30.7 \\
\end{array}$ & $\begin{array}{l}33.8 \\
28.1 \\
\end{array}$ & $\begin{array}{l}34.3 \\
28.8 \\
\end{array}$ \\
\hline 10. One Flygt mixer & 75 & $\begin{array}{l}40.0 \\
40.8 \\
\end{array}$ & $\begin{array}{l}40.0 \\
40.8 \\
\end{array}$ & $\begin{array}{l}37.5 \\
37.7 \\
\end{array}$ & $\begin{array}{l}37.5 \\
37.7 \\
\end{array}$ \\
\hline 11. Two Flygt Mixers & 50 & $\begin{array}{l}38.1 \\
35.6 \\
\end{array}$ & $\begin{array}{l}38.1 \\
35.6 \\
\end{array}$ & $\begin{array}{l}35.6 \\
32.8 \\
\end{array}$ & $\begin{array}{l}35.9 \\
33.2 \\
\end{array}$ \\
\hline 12. One Slurry Pump & 50 & $\begin{array}{l}38.0 \\
42.2 \\
\end{array}$ & $\begin{array}{l}39.7 \\
43.7 \\
\end{array}$ & $\begin{array}{l}37.3 \\
41.1\end{array}$ & $\begin{array}{l}39.7 \\
43.7\end{array}$ \\
\hline 13. Three Flygt Mixers & 50 & $\begin{array}{l}38.1 \\
36.2 \\
\end{array}$ & $\begin{array}{l}38.1 \\
36.2 \\
\end{array}$ & $\begin{array}{l}35.8 \\
33.5\end{array}$ & $\begin{array}{l}36.2 \\
34.0\end{array}$ \\
\hline 14. Two Flygt Mixers & 75 & $\begin{array}{l}40.2 \\
41.3\end{array}$ & $\begin{array}{l}40.2 \\
41.3\end{array}$ & $\begin{array}{l}37.5 \\
38.1\end{array}$ & $\begin{array}{l}37.7 \\
38.3\end{array}$ \\
\hline 15. One Slurry Pump & $\overline{75}$ & $\begin{array}{l}40.1 \\
47.3\end{array}$ & $\begin{array}{l}41.2 \\
47.3 \\
\end{array}$ & $\begin{array}{l}38.9 \\
45.6\end{array}$ & $\begin{array}{l}41.2 \\
47.9\end{array}$ \\
\hline 16. Three Flygt Mixers & 75 & $\begin{array}{l}40.2 \\
42.2\end{array}$ & $\begin{array}{l}40.2 \\
42.2\end{array}$ & $\begin{array}{l}37.8 \\
39.3\end{array}$ & $\begin{array}{l}38.2 \\
39.6\end{array}$ \\
\hline
\end{tabular}




\section{Decant}

Decant of Existing. Supemate from TK 37 to TK 30

Before Batch Dissolution Prcessses of Tank 37

(Salt Solution to be cransferred to TK 26 Later)

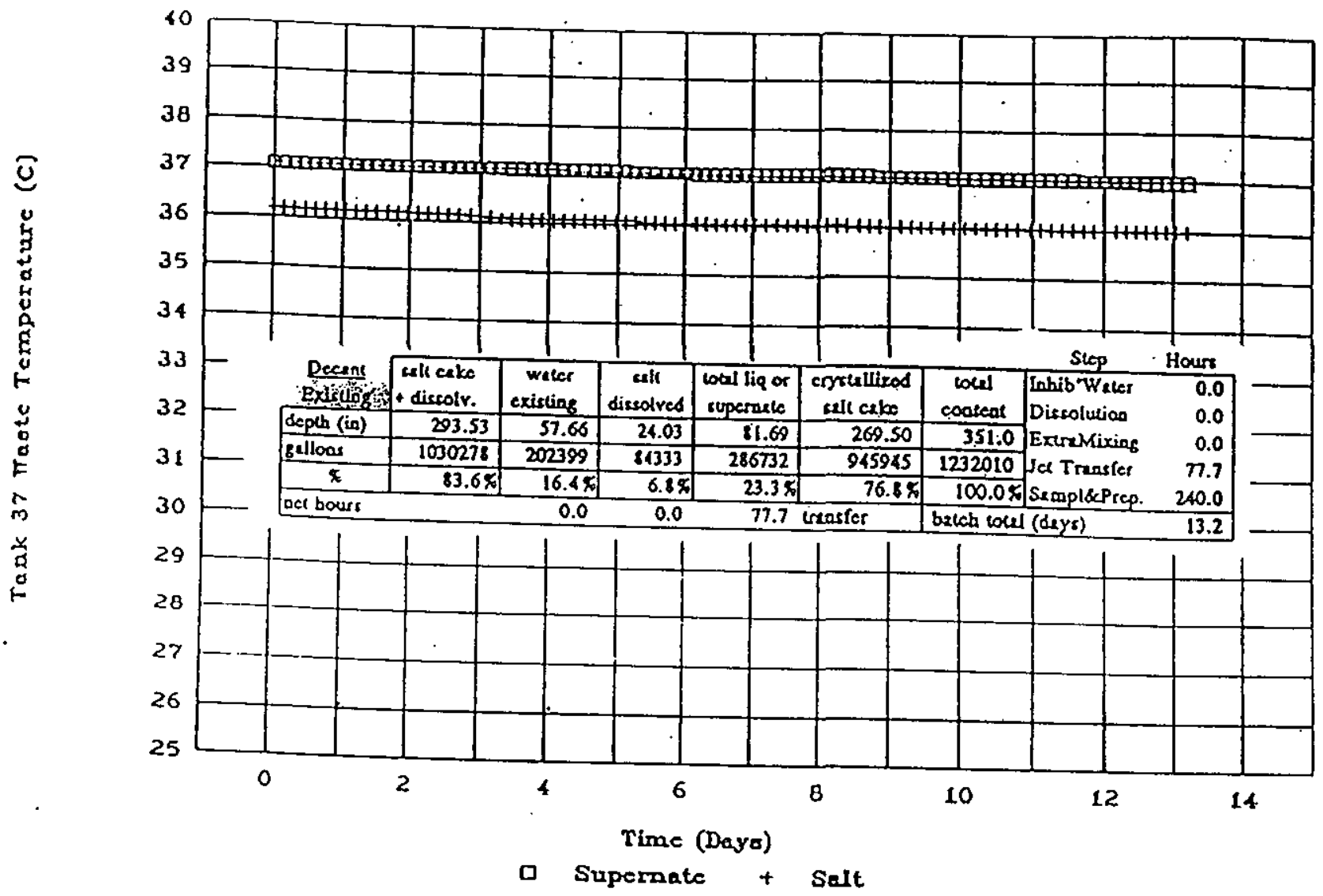




\section{Case 1: No Agitation with 25C IW (Batch 1-2)}
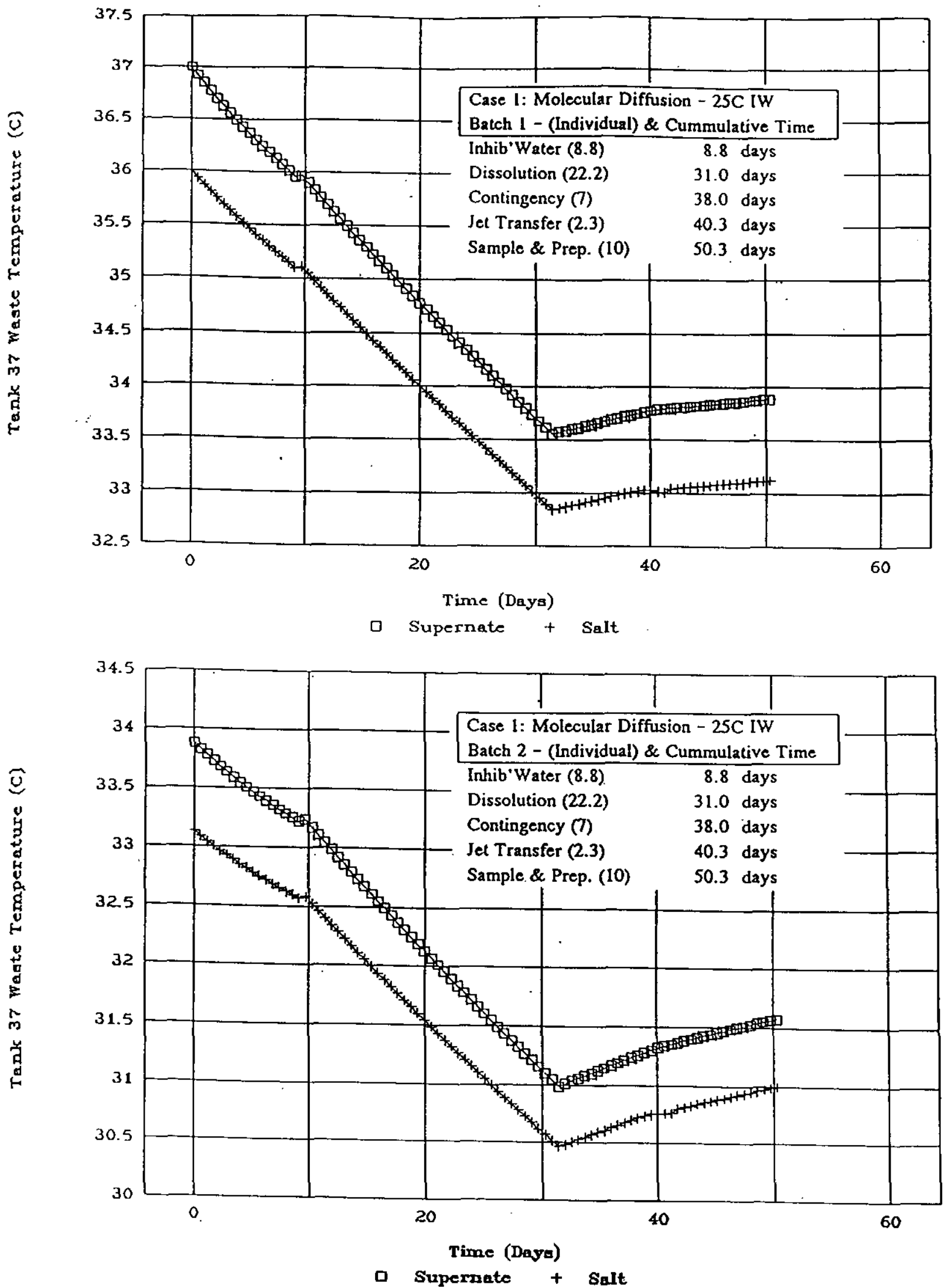
Case 1: No Agitation with 25C IW (Batch 3-4)
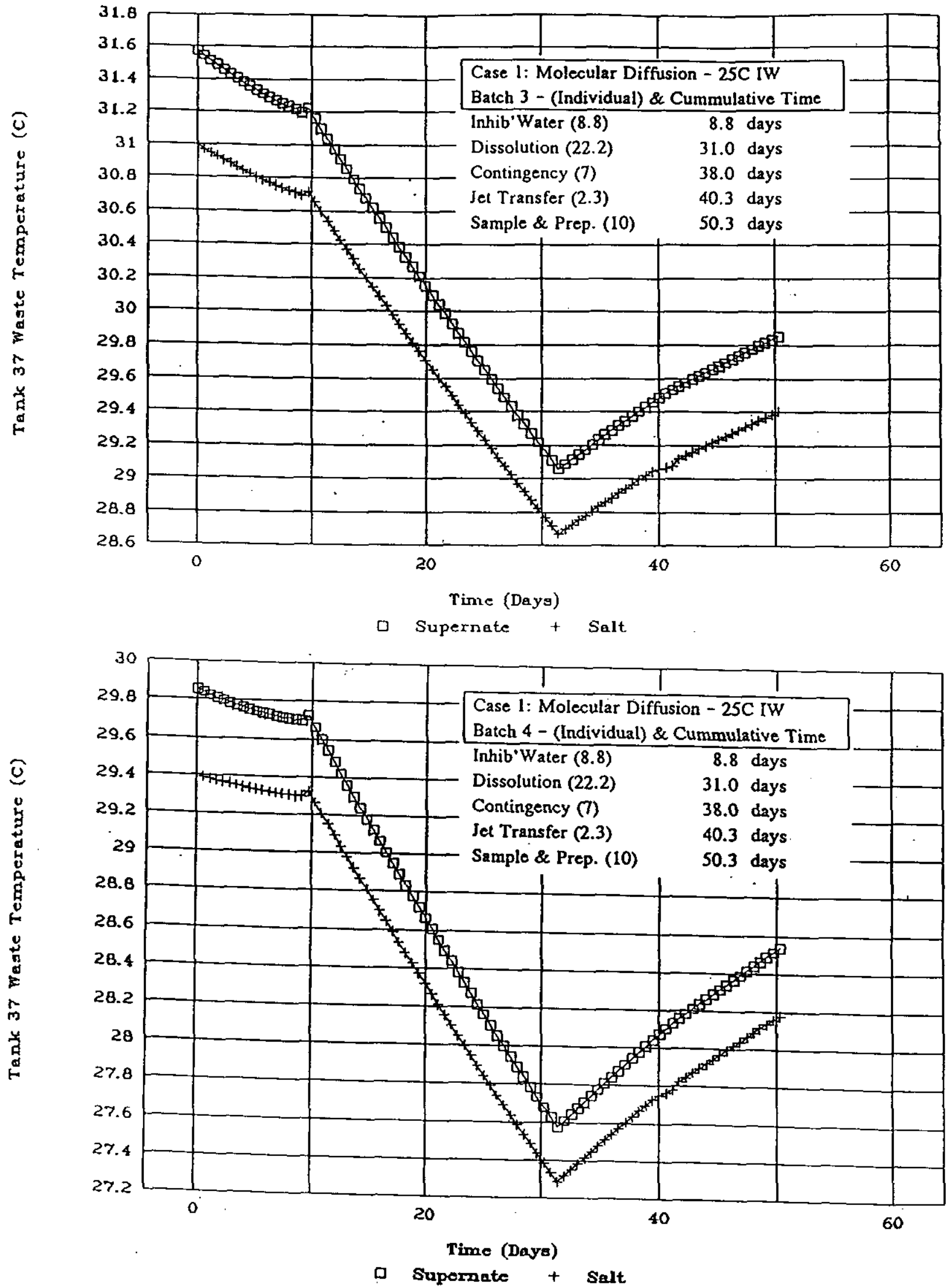
Case 2: Modified Density Gradient (Batch 1-2)
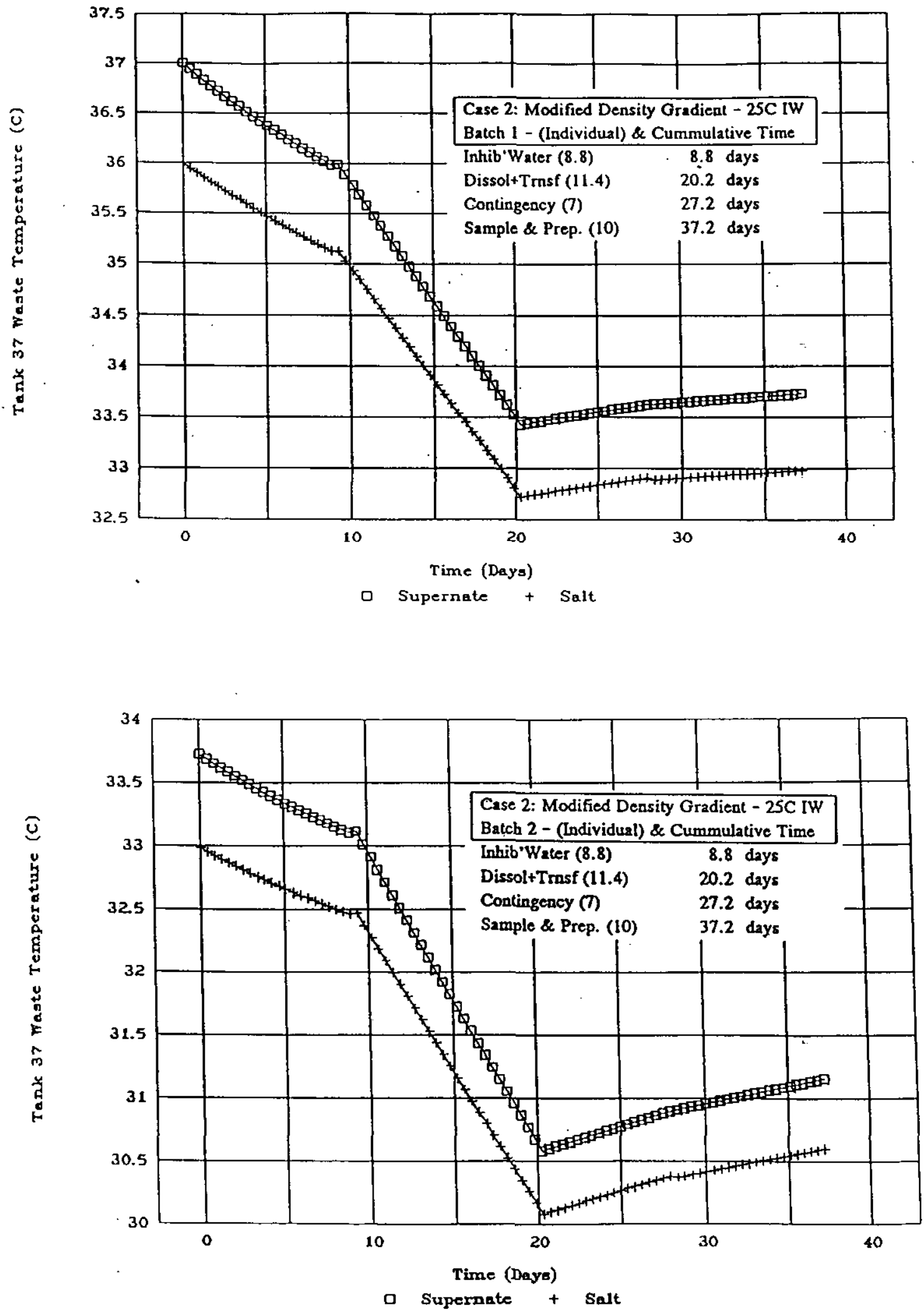
Case 2: Modified Density Gradient (Batch 3-4)
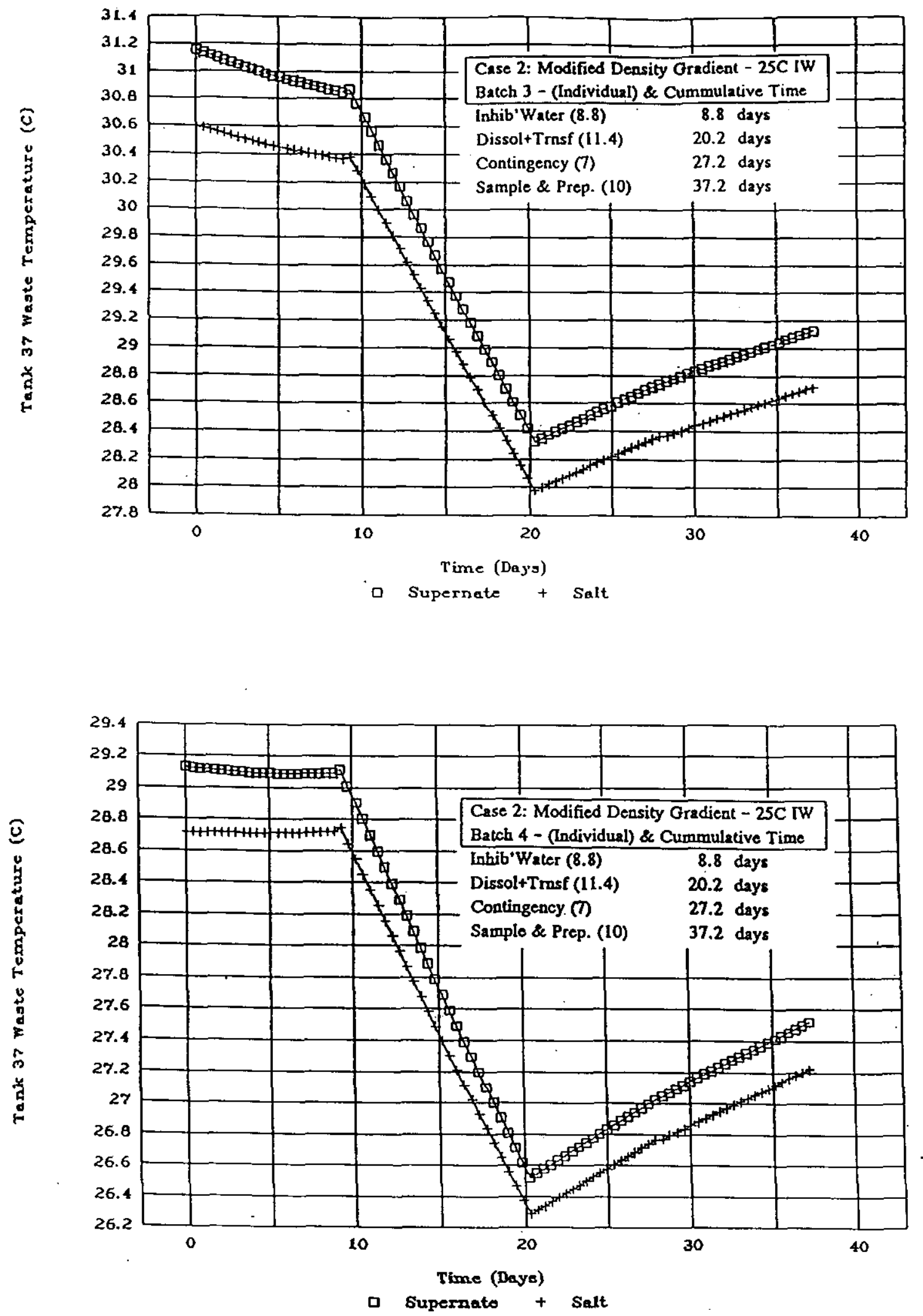
Case 3: Modified Density Gradient-50C IW (Batch 1-2)
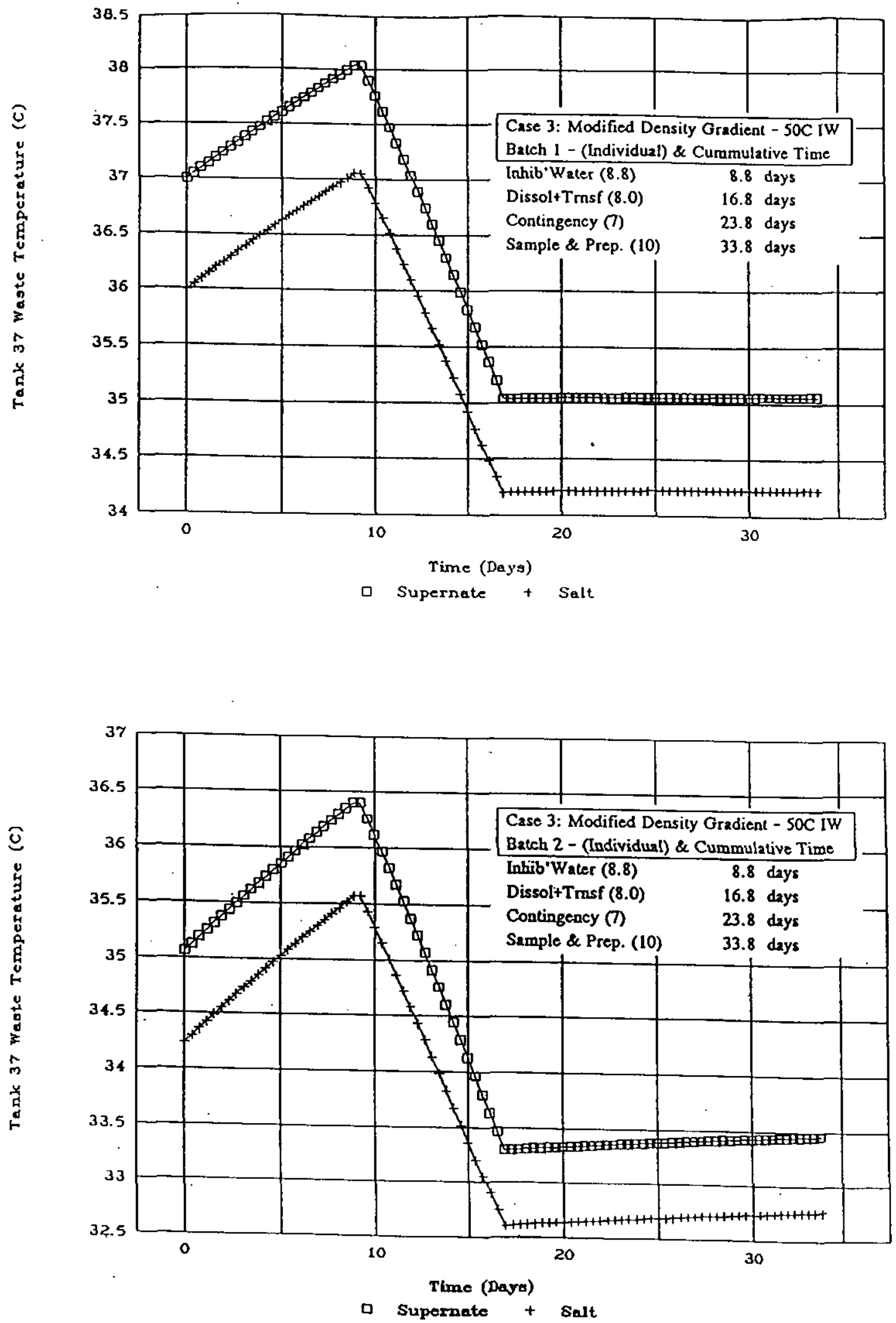
Case 3: Modified Density Gradient-50C IW (Batch 3-4)
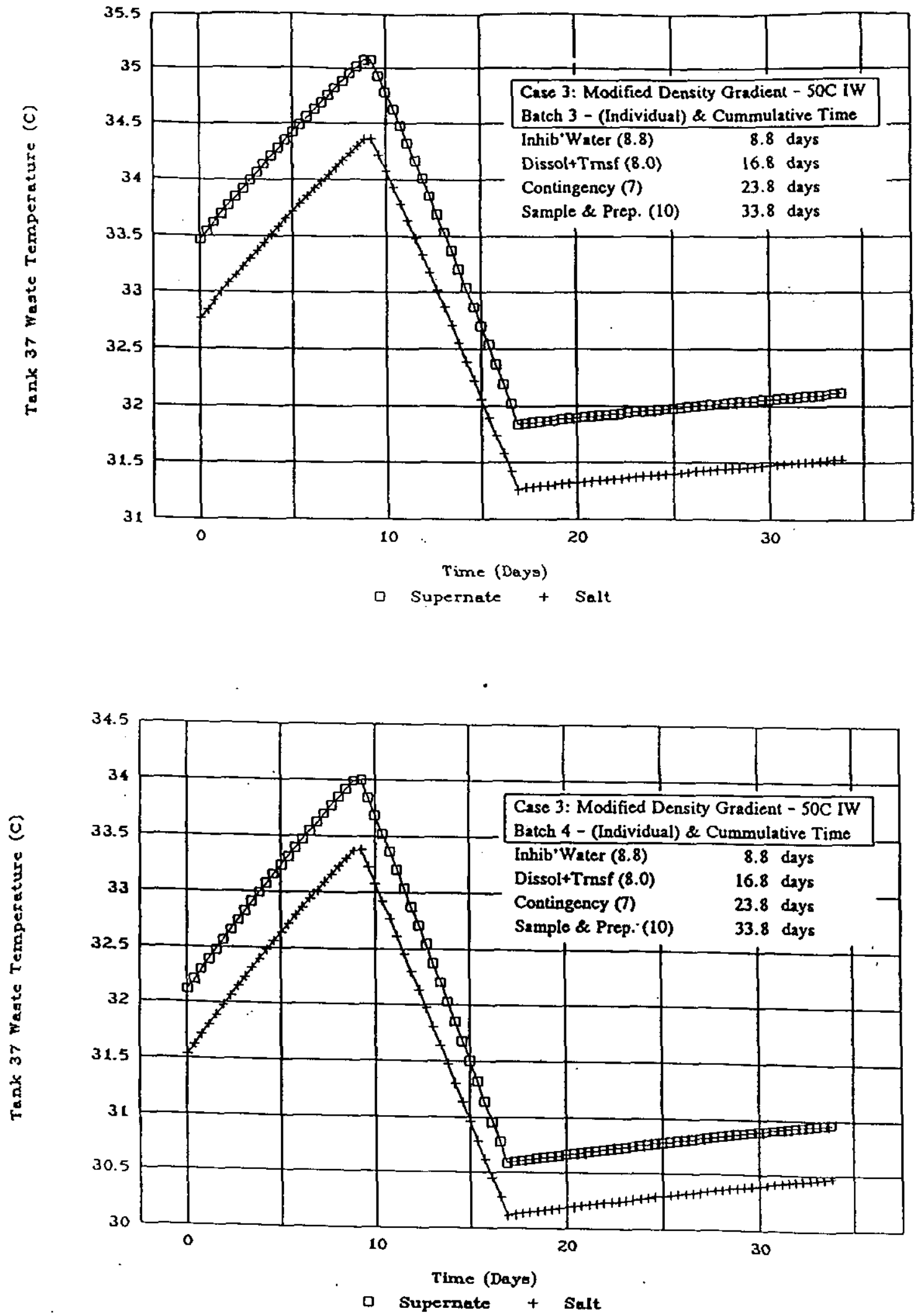
Case 4: Modified Density Gradient-75C IW (Batch 1-2)
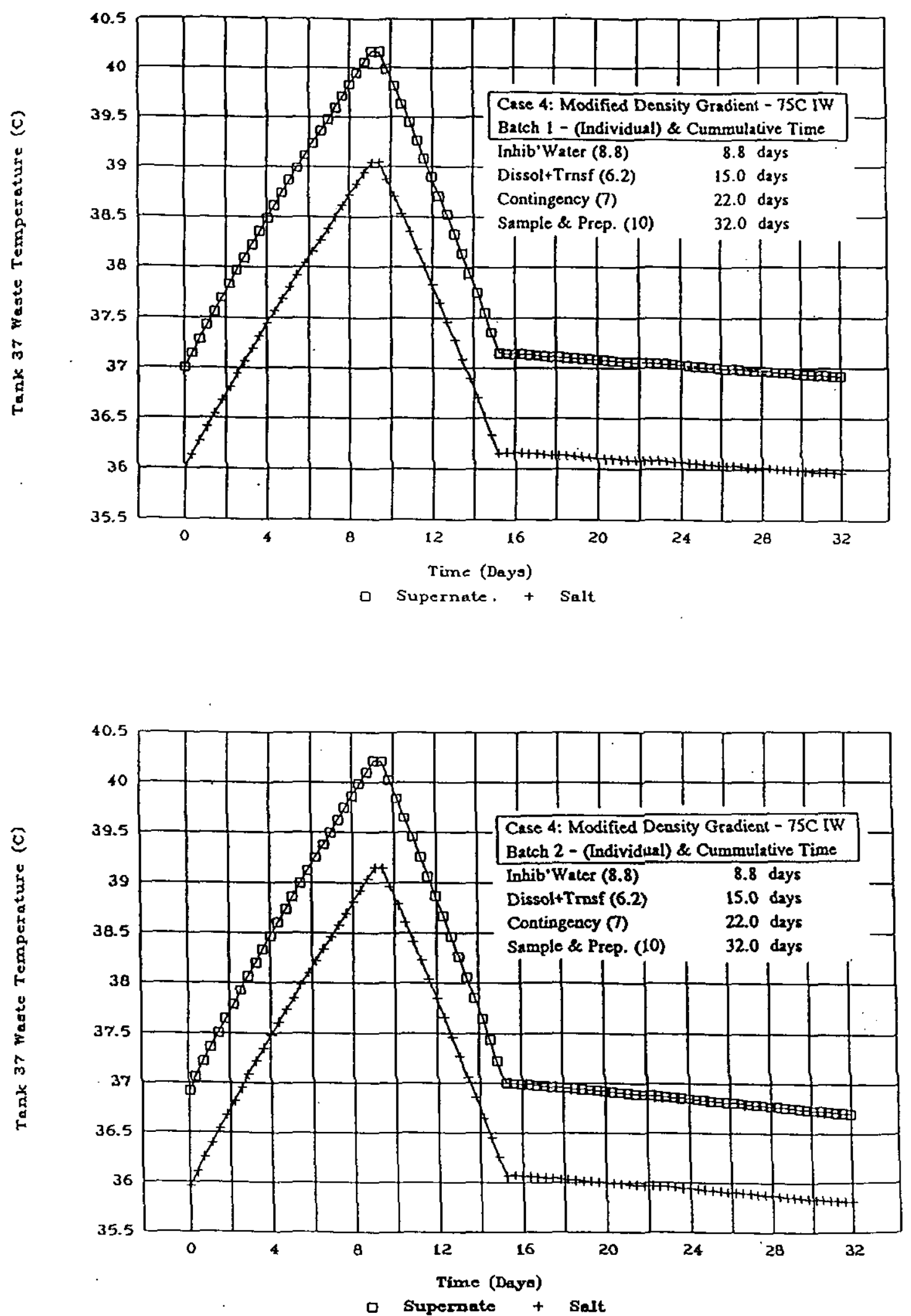
Case 4: Modified Density Gradient-75C IW (Batch 3-4)
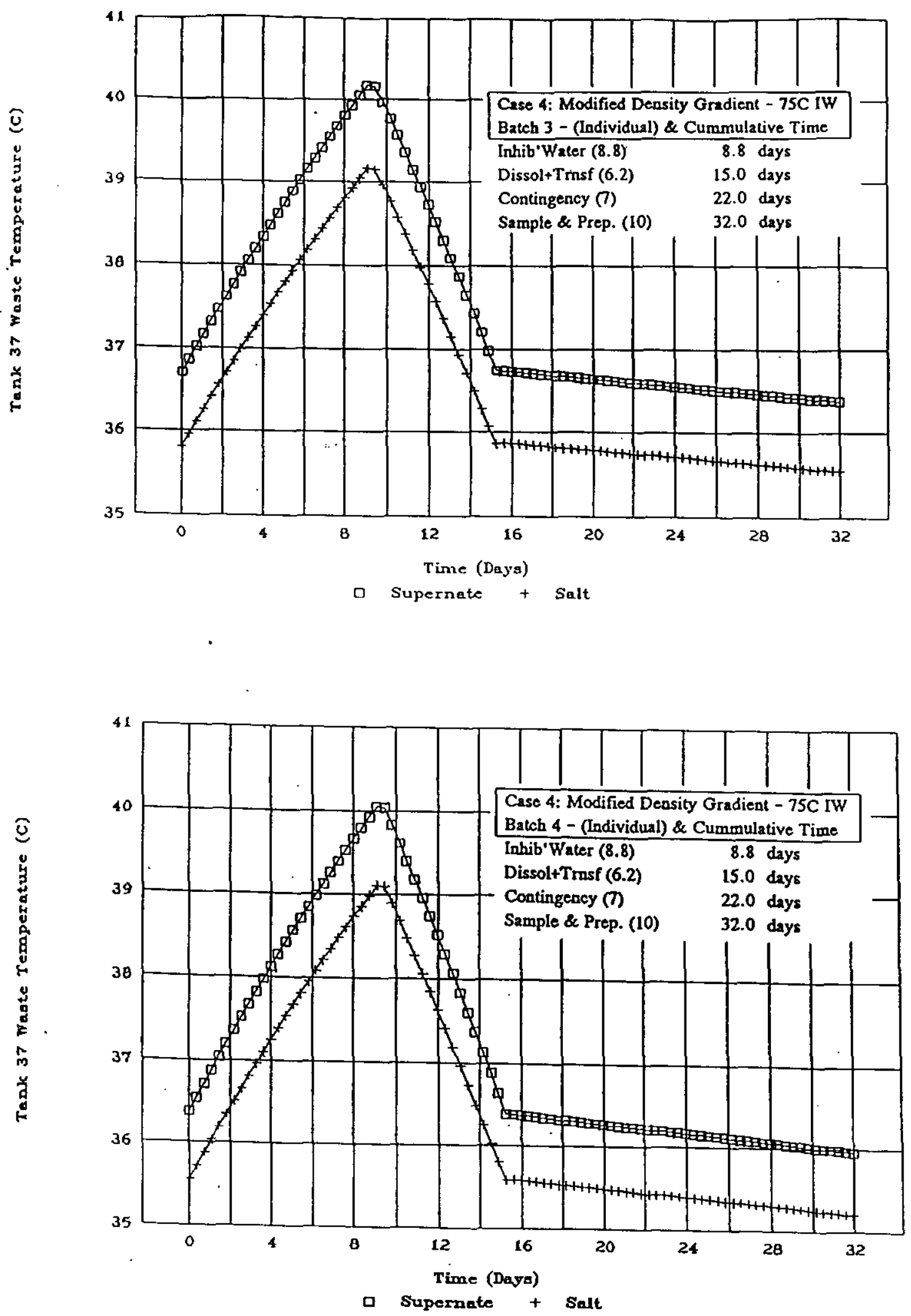
Case 5: One Flygt Mixer with 25C. IW (Batch 1-2)
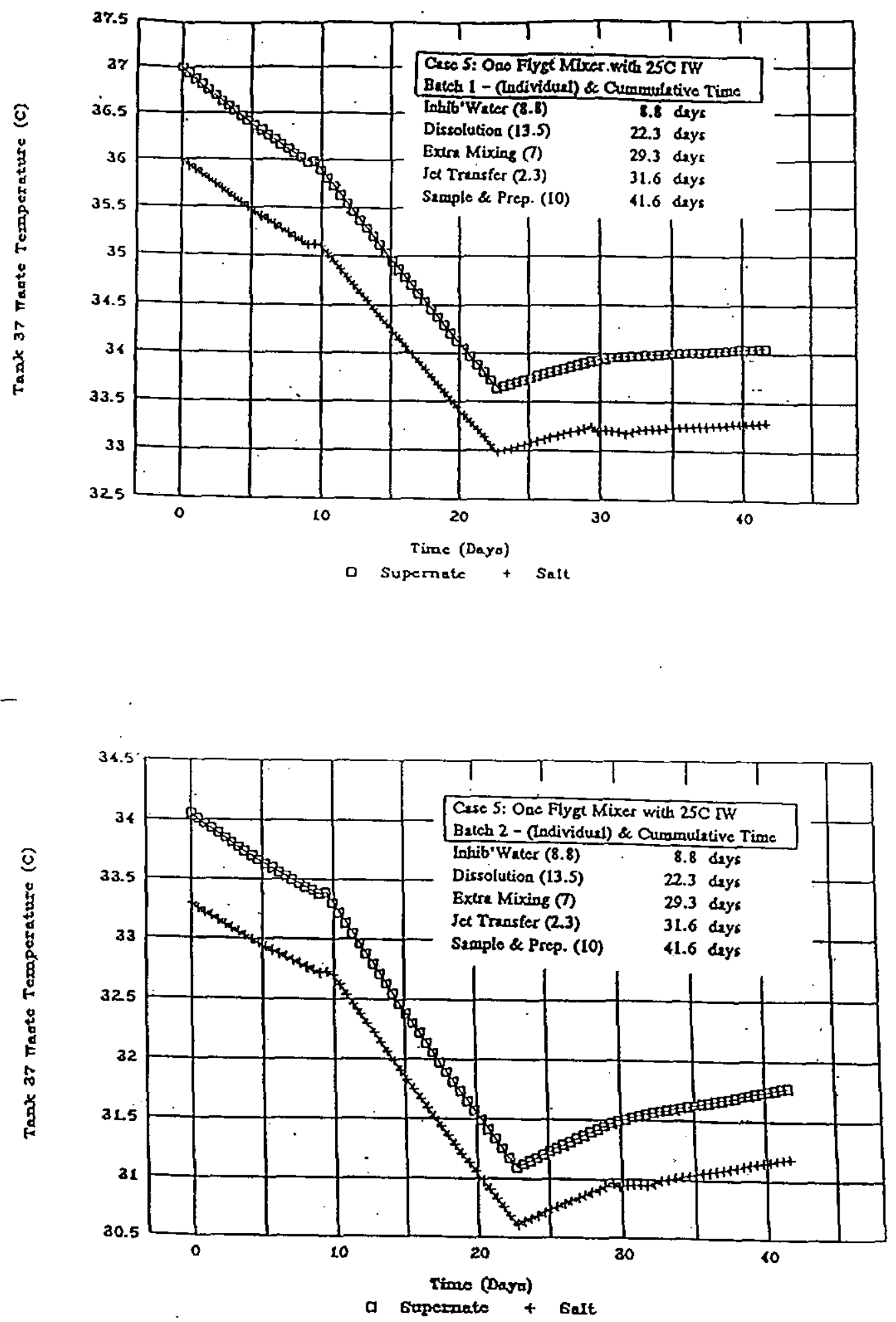
Case 5: One Flygt Mixer with 25C IW (BATCH 3-4)
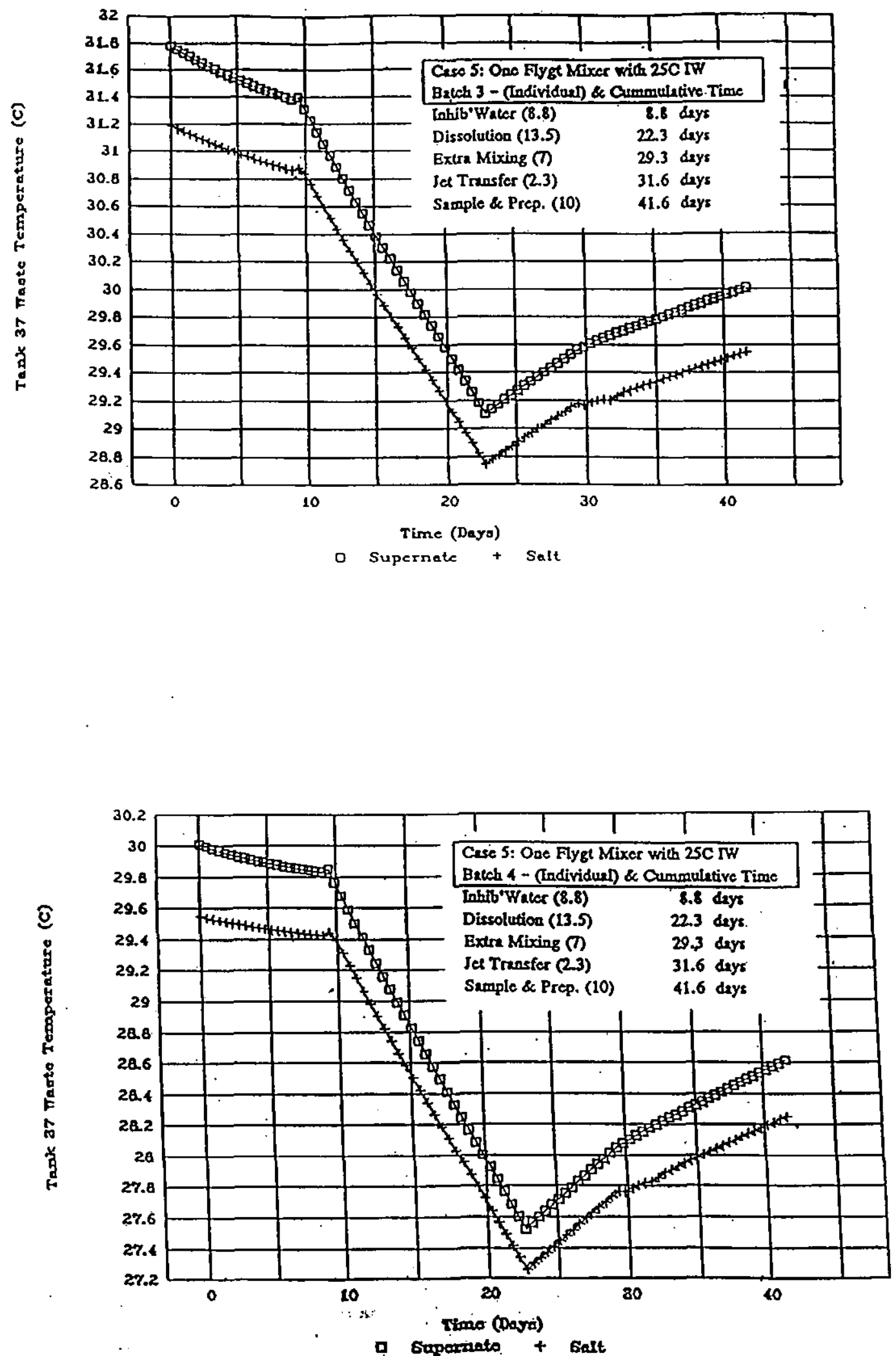

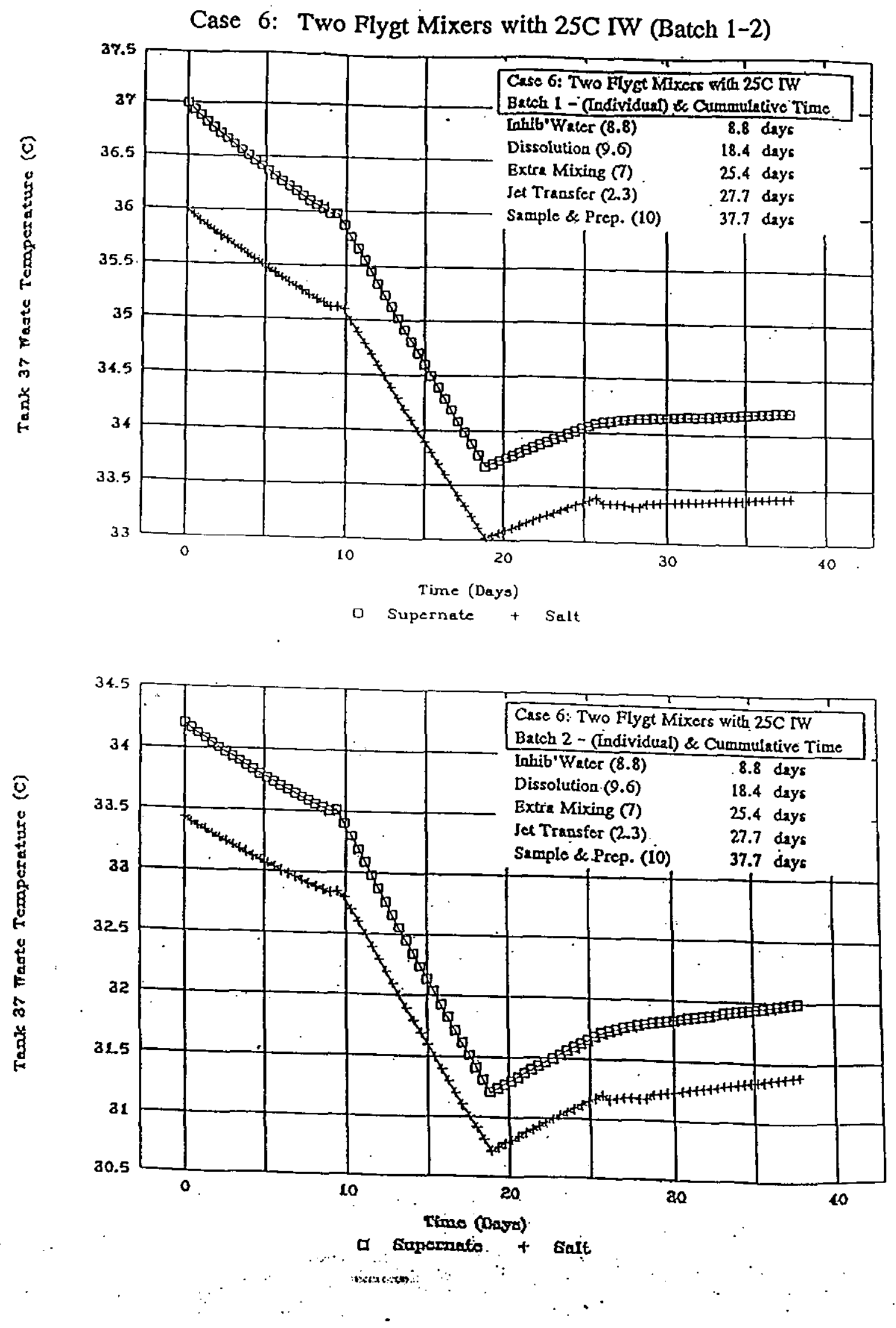


\section{Case 6: Two Flygt Mixers with 25C IW (Batch 3-4)}
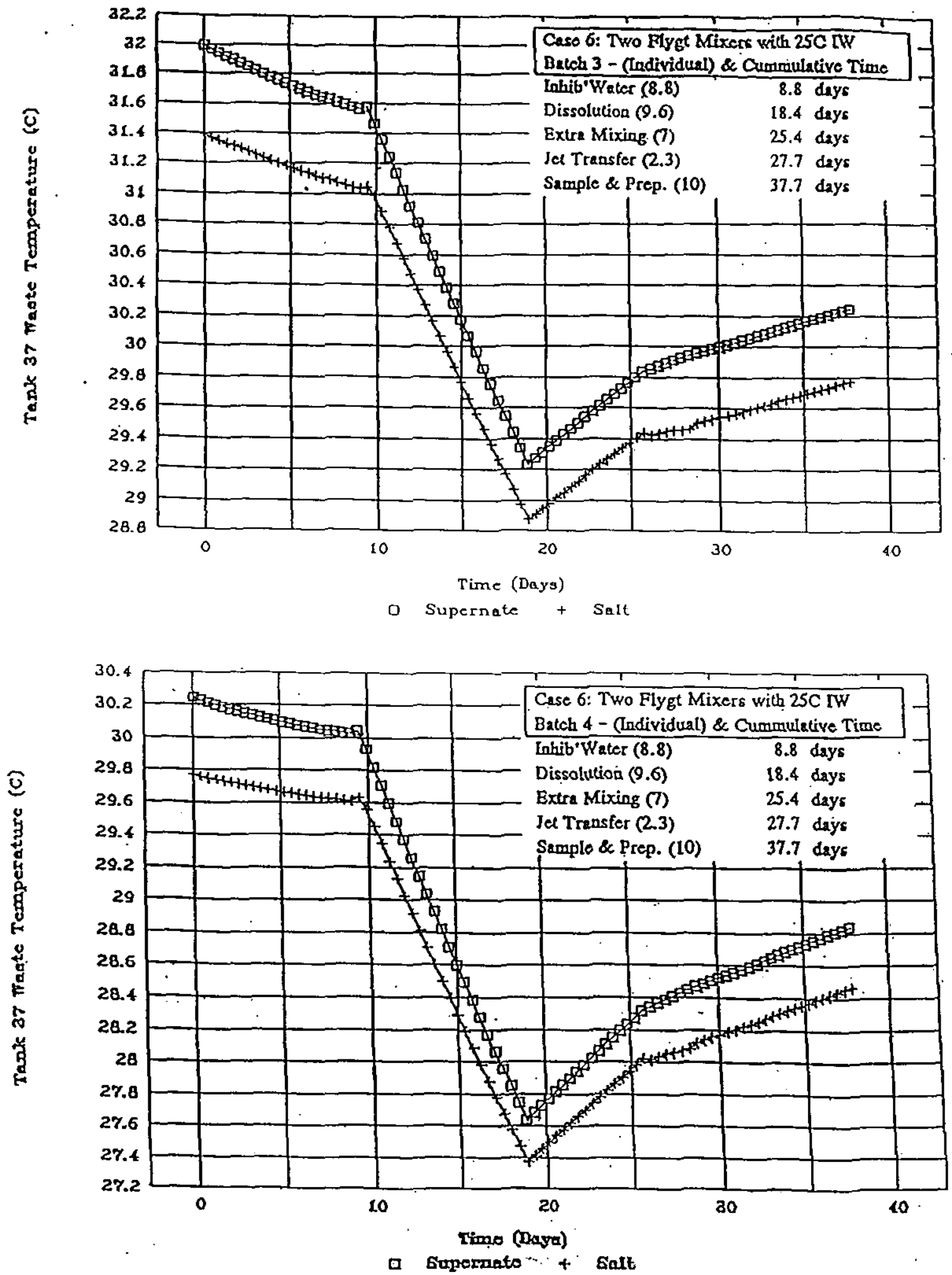
Case 7: One Flygt Mixer with 50C IW (Batch 1-2)
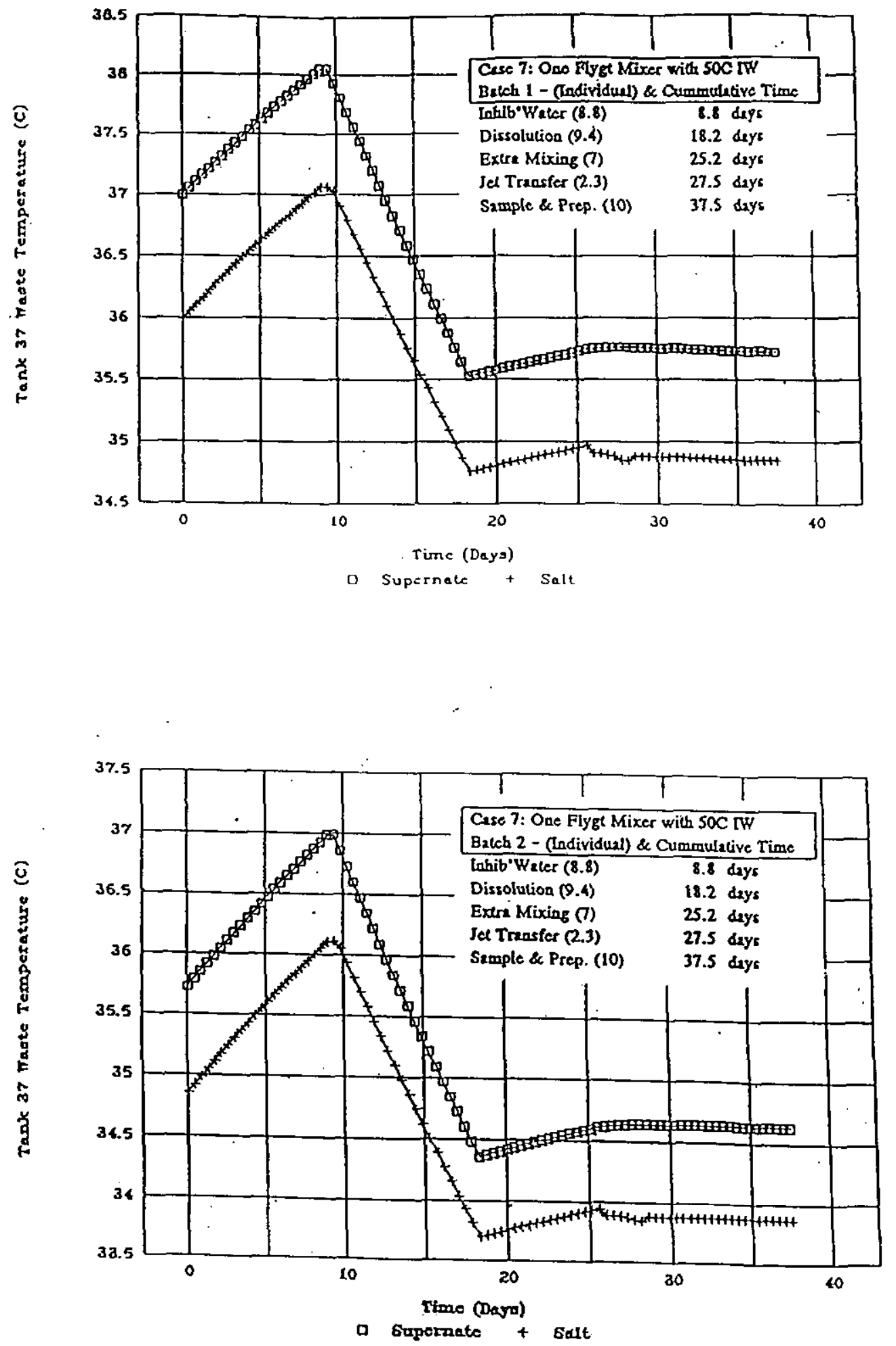
Case 7: One Flygt Mixer with 50C IW (Batch 3-4)
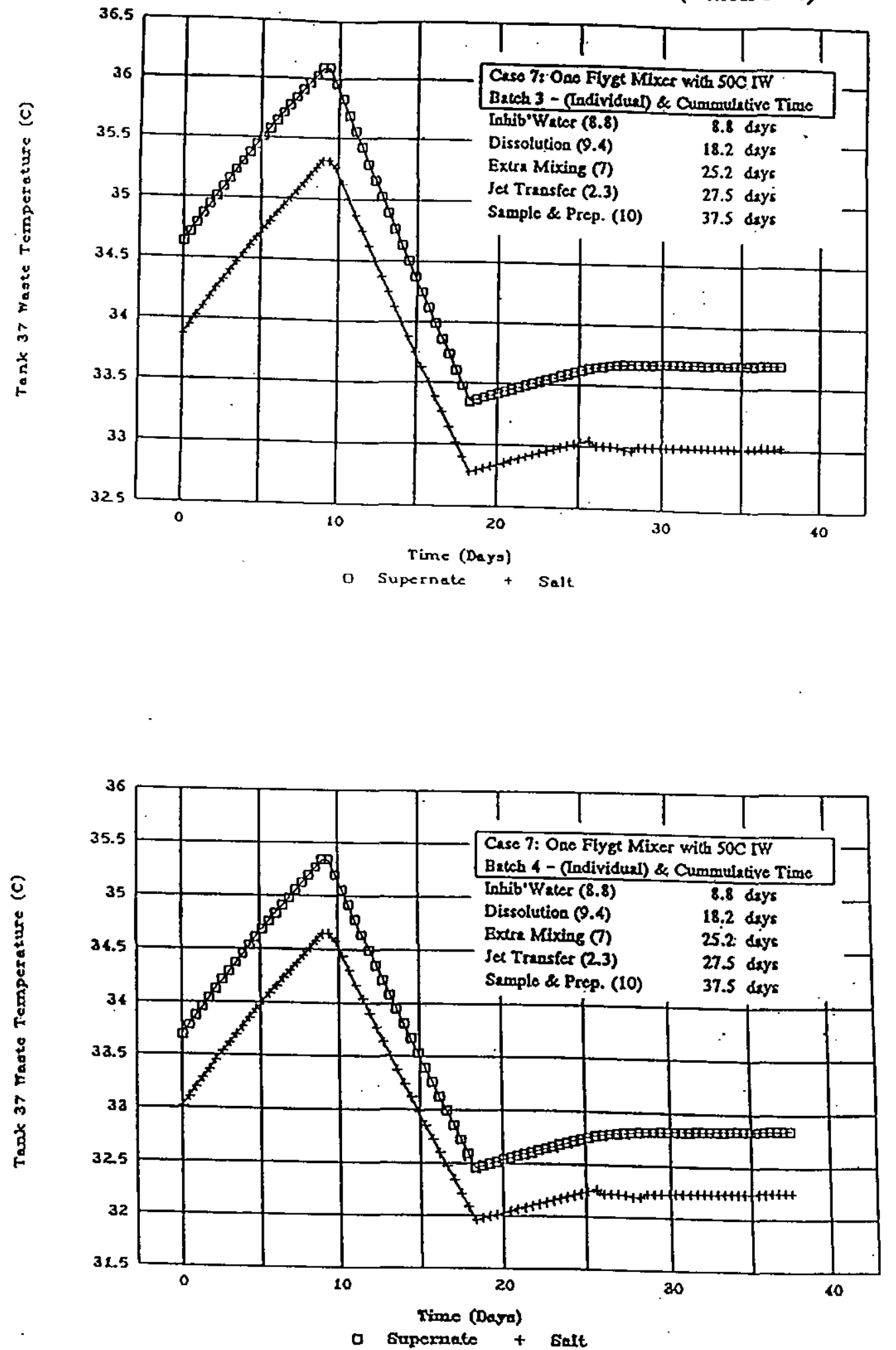
Case 8: One Slurry Pump with 25C IW (Batch 1-2)
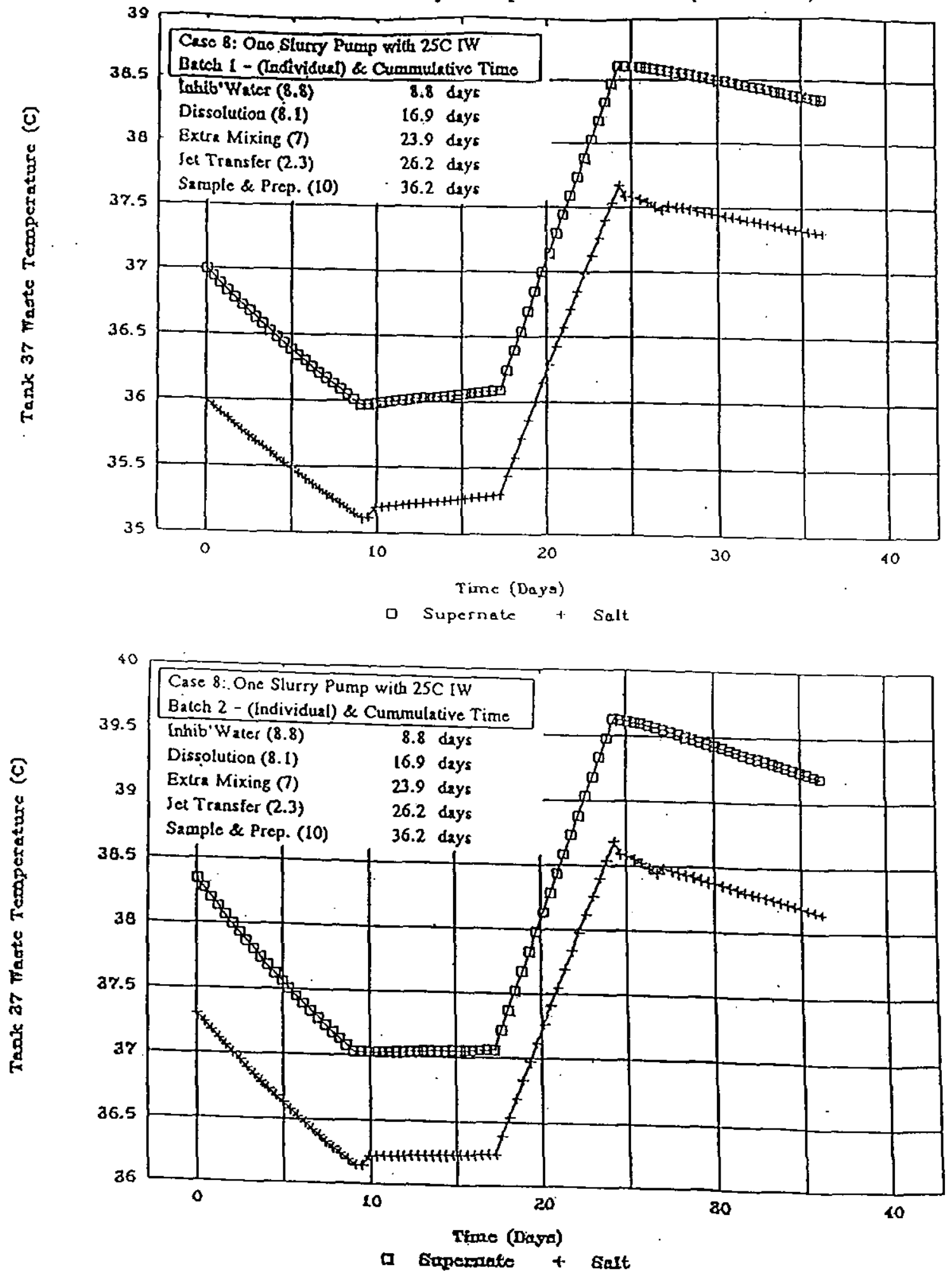
Case 8: One Slurry Pump with 25C IW (Batch 3-4)

Caso 8: Ono Siurty Ramp with 25C IW

Batch 3 - Gndividual) \& Cummulative Time
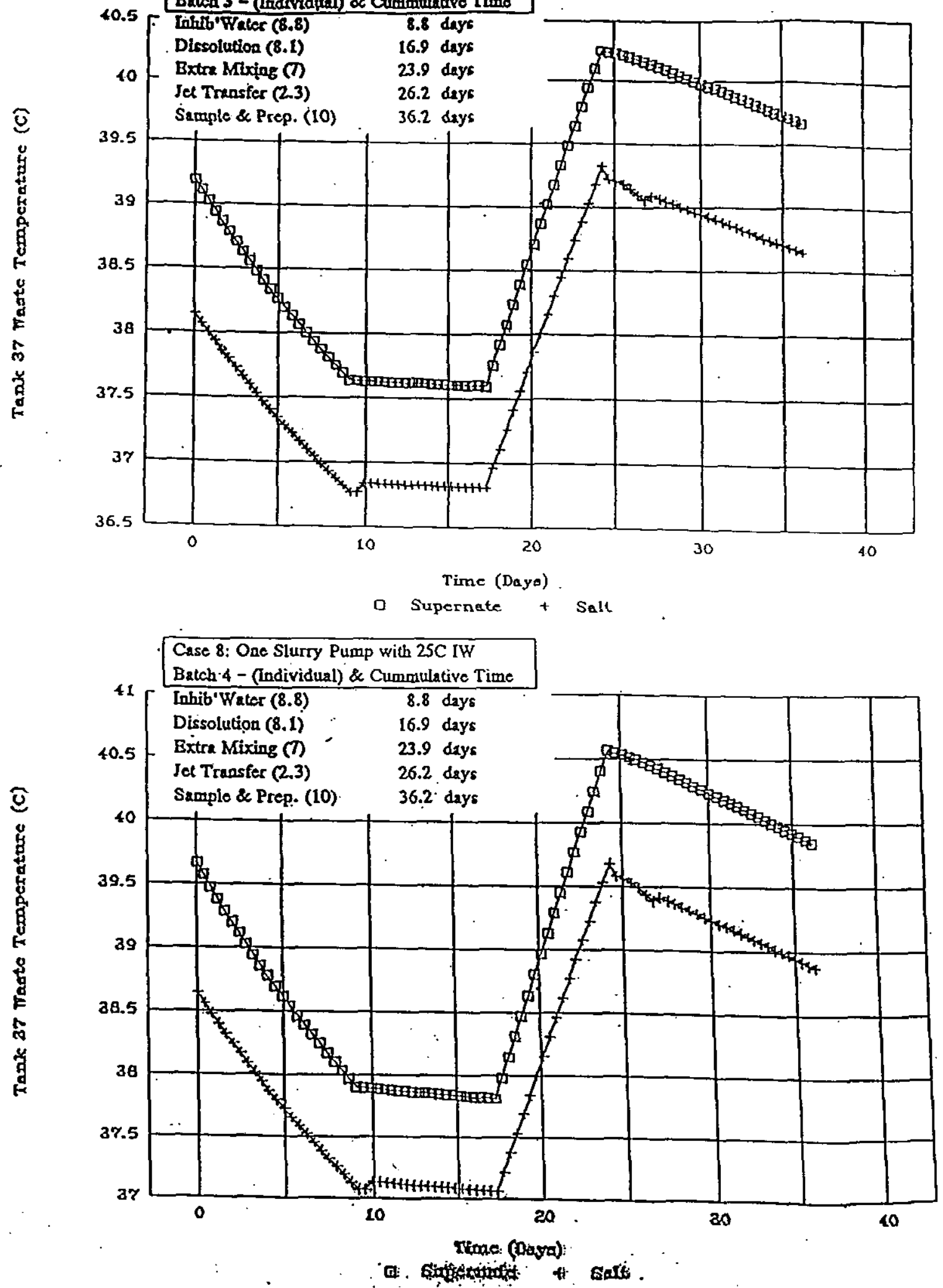
Case 9: Three Flygt Mixers with 25C IW (Batch 1-2)
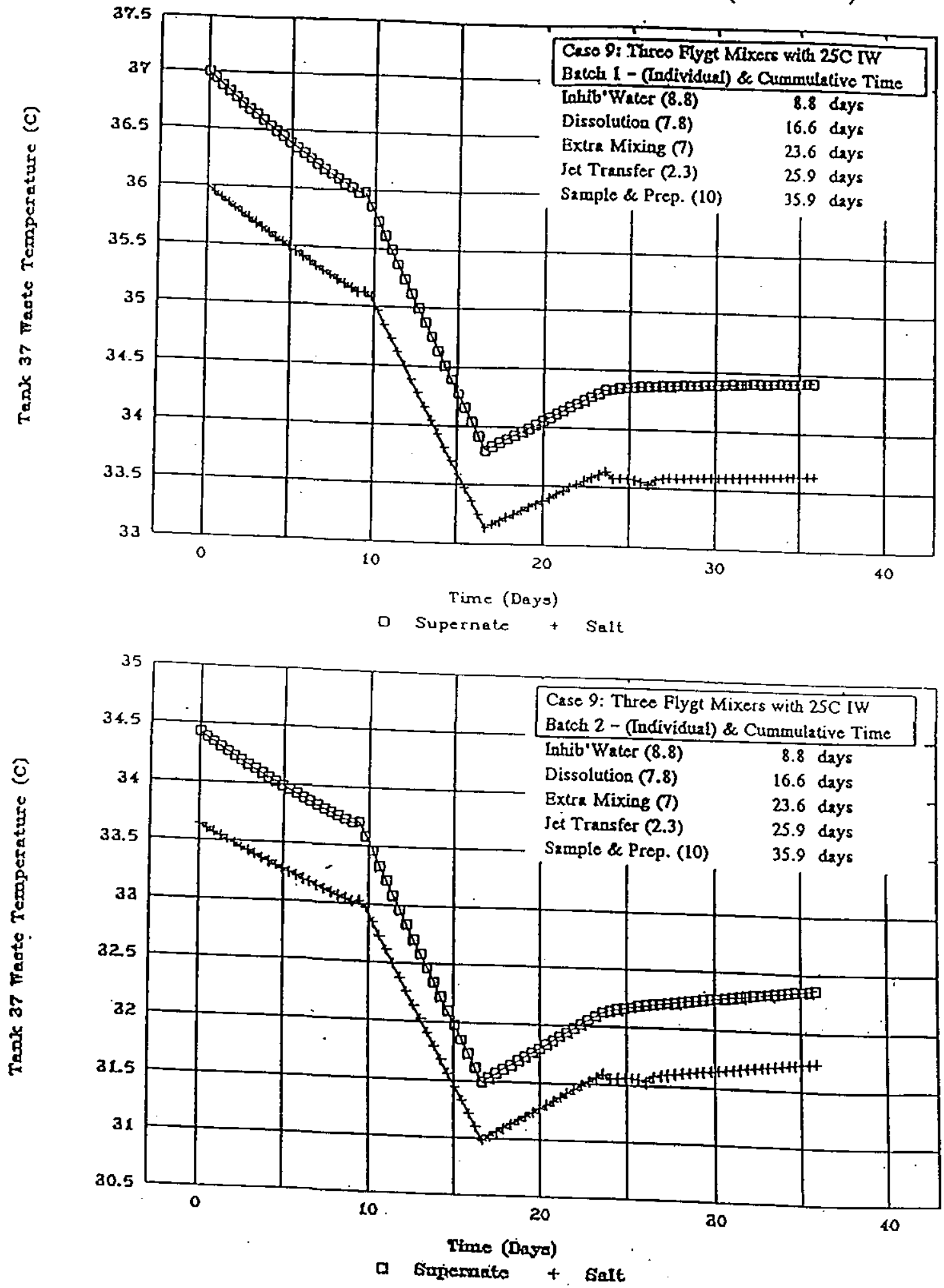
Case 9: Three Flygt Mixers with 25C IW (Batch 3-4)
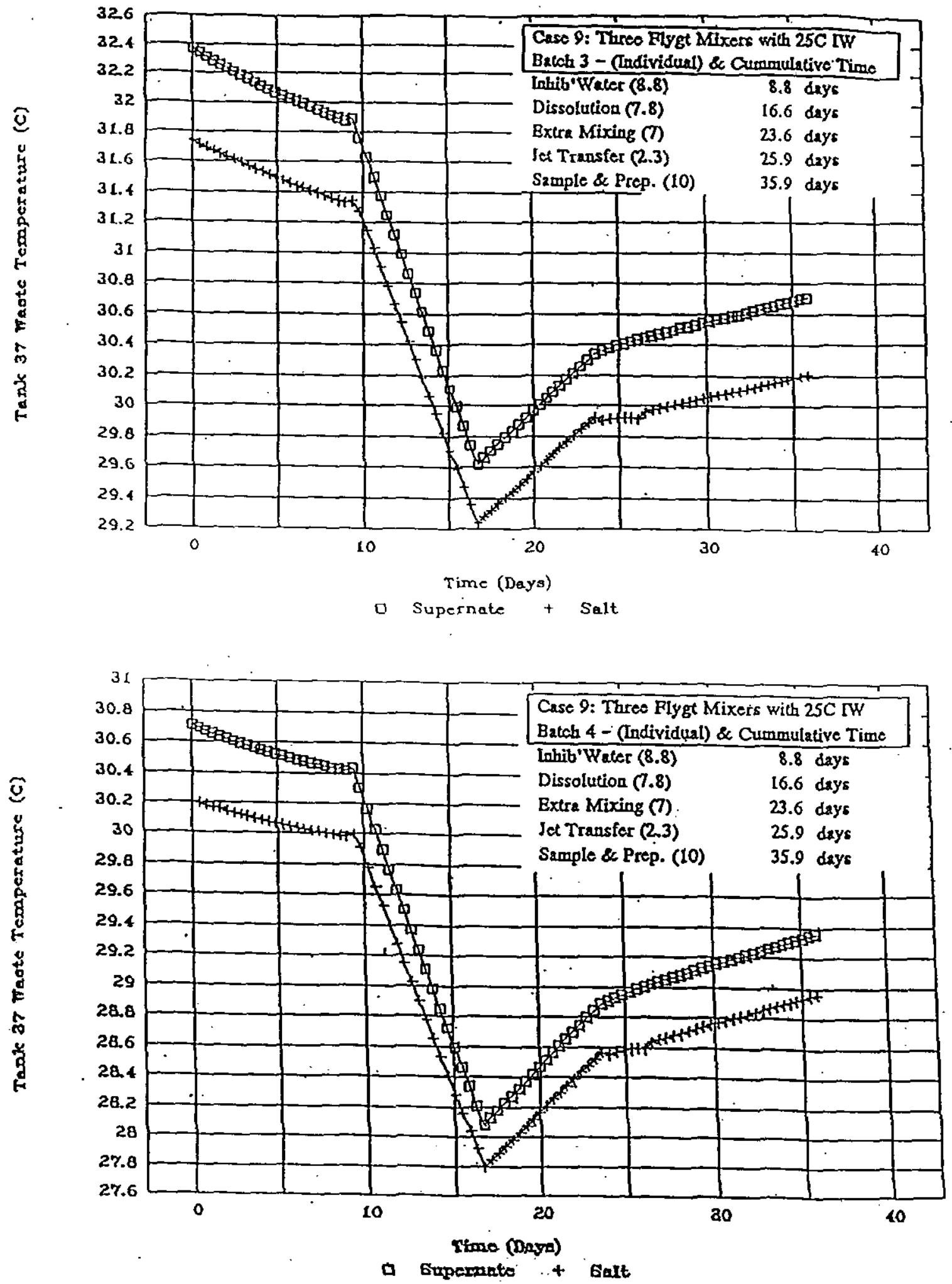
Case 10: One Flygt Mixer with 75C IW (Batch 1-2)
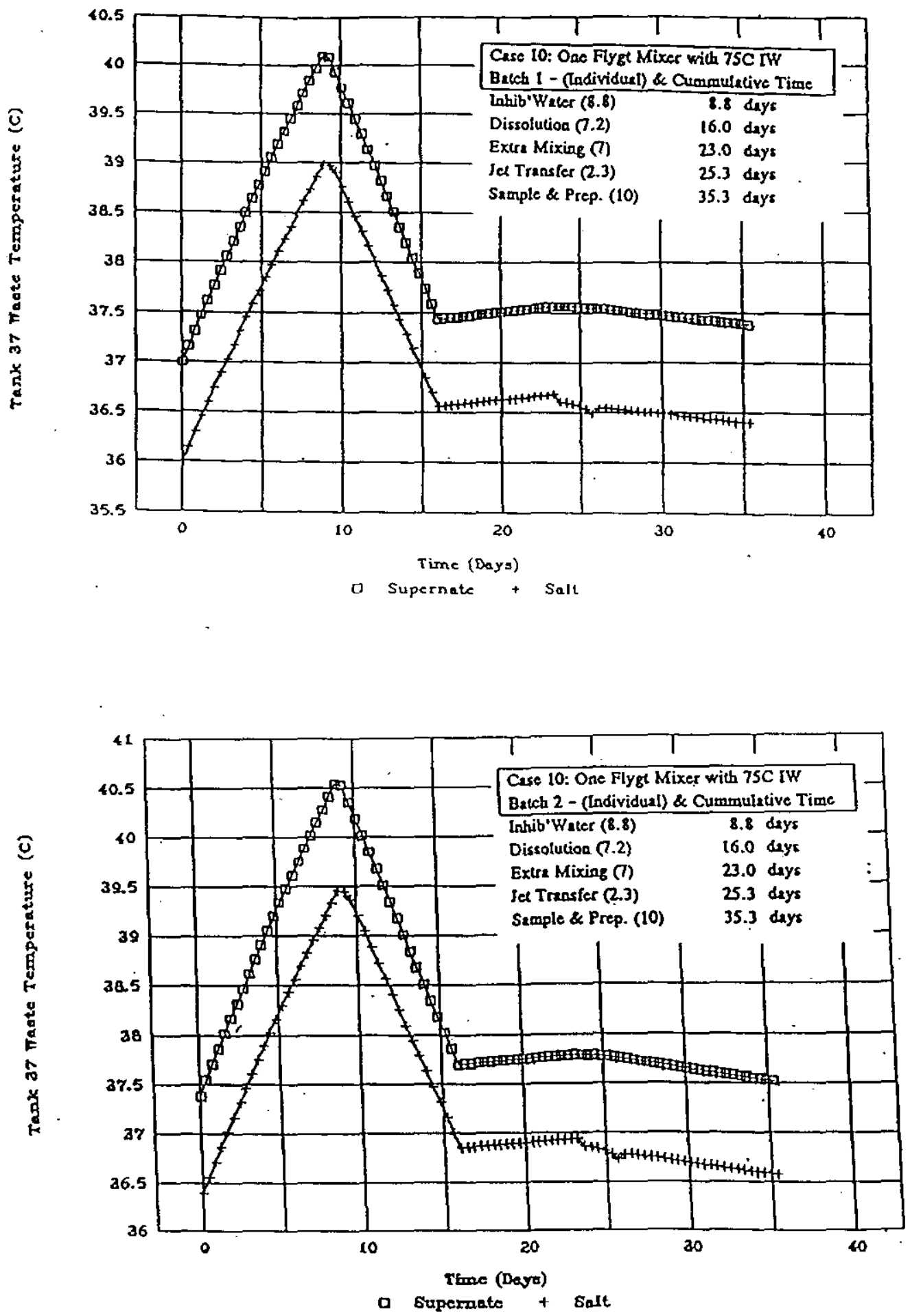
Case 10 One Flygt Mixer with 75C IW (Batch 3-4)
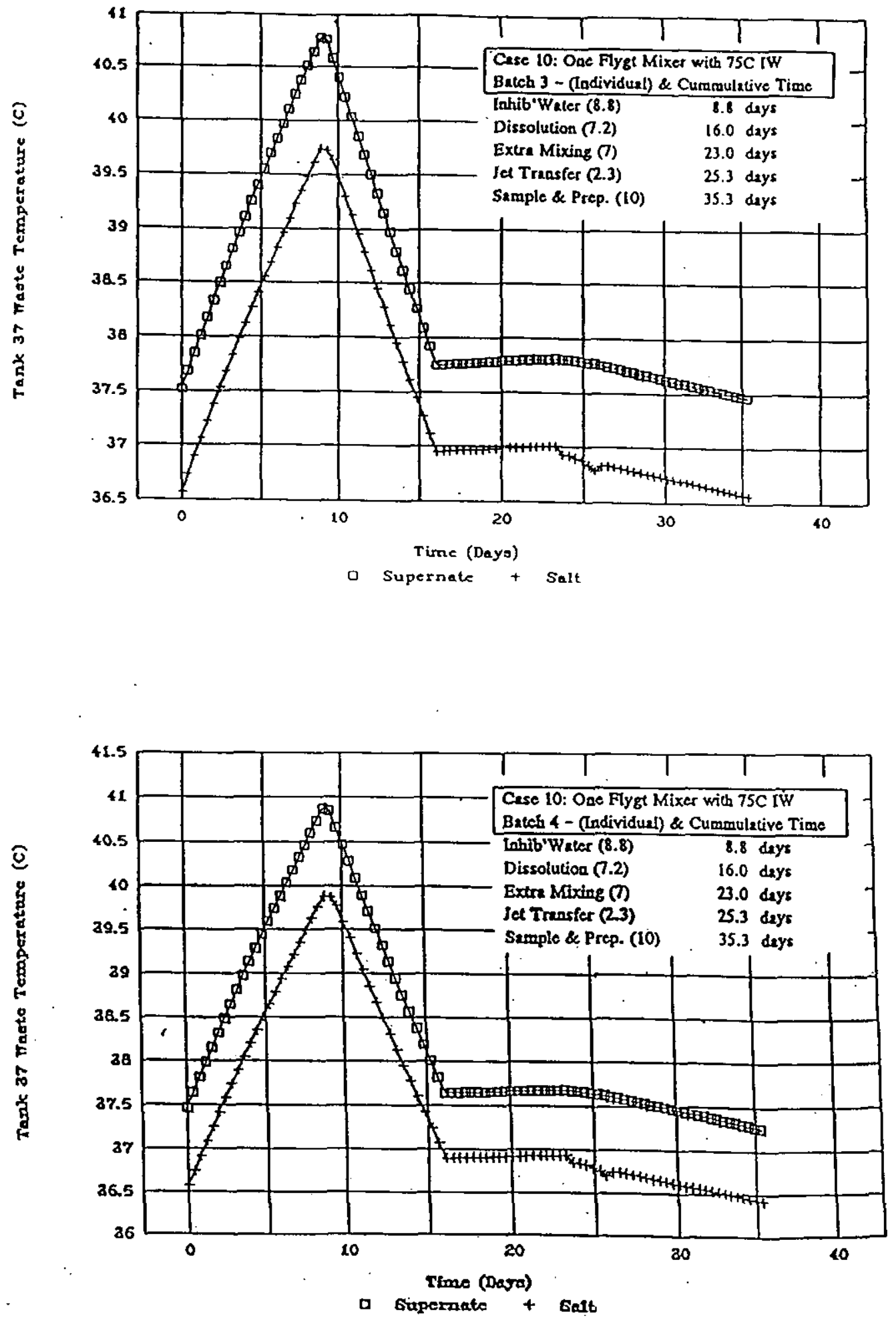

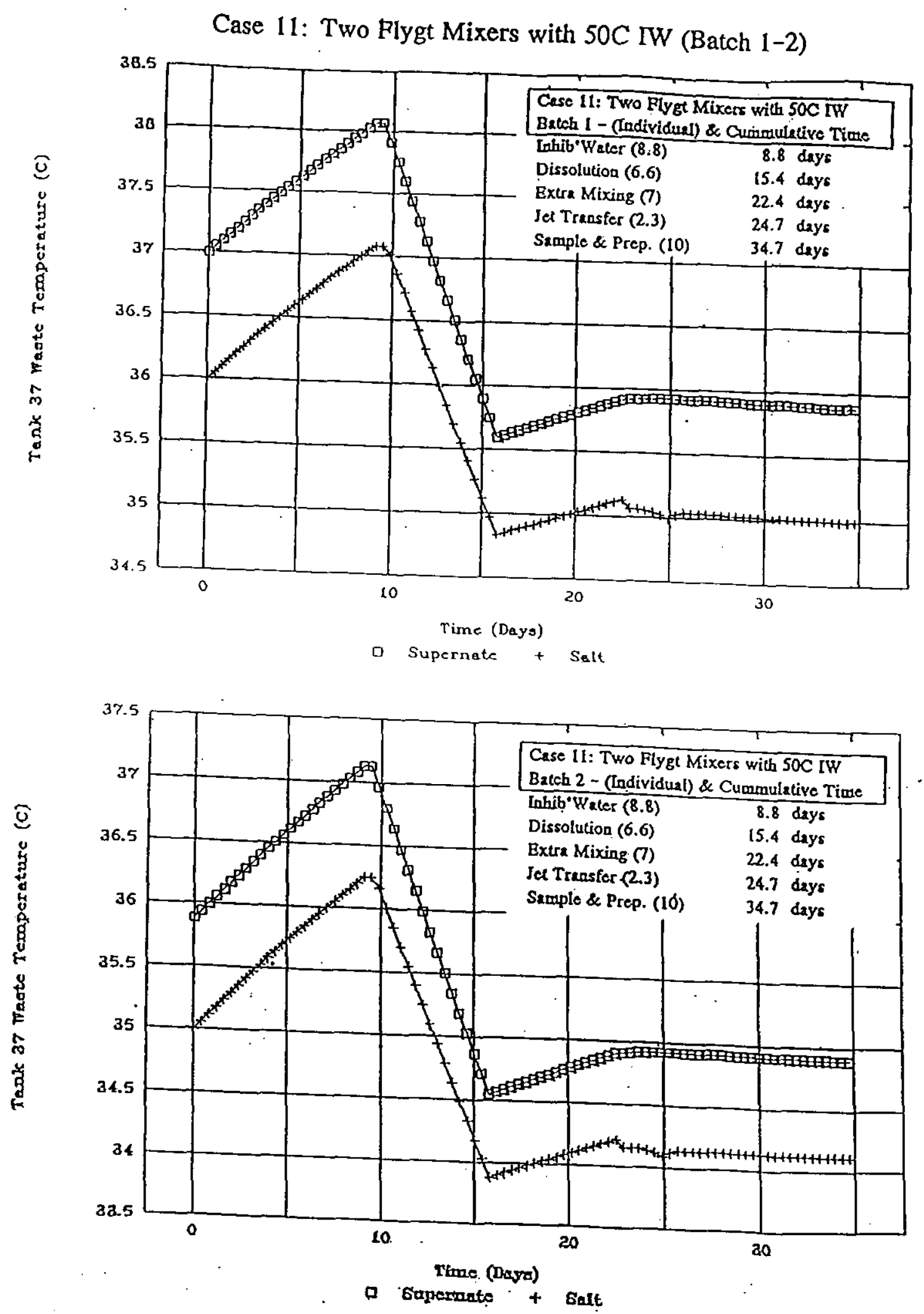
Case 11 Two Plygt Mixers with 50C IW (Batch 3-4)
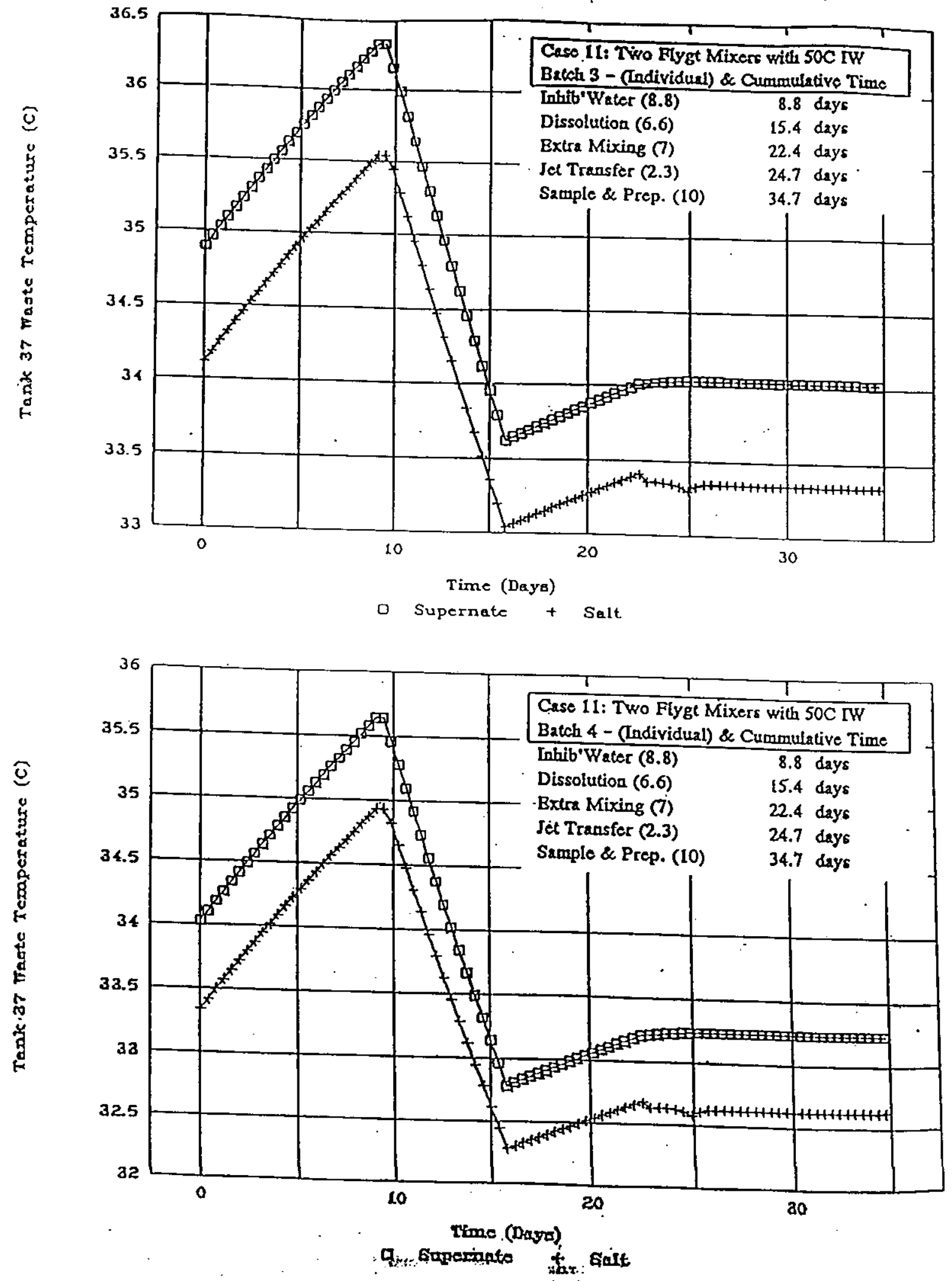
Case 12: One Slurry Pumps with 50C IW (Batch 1-2)
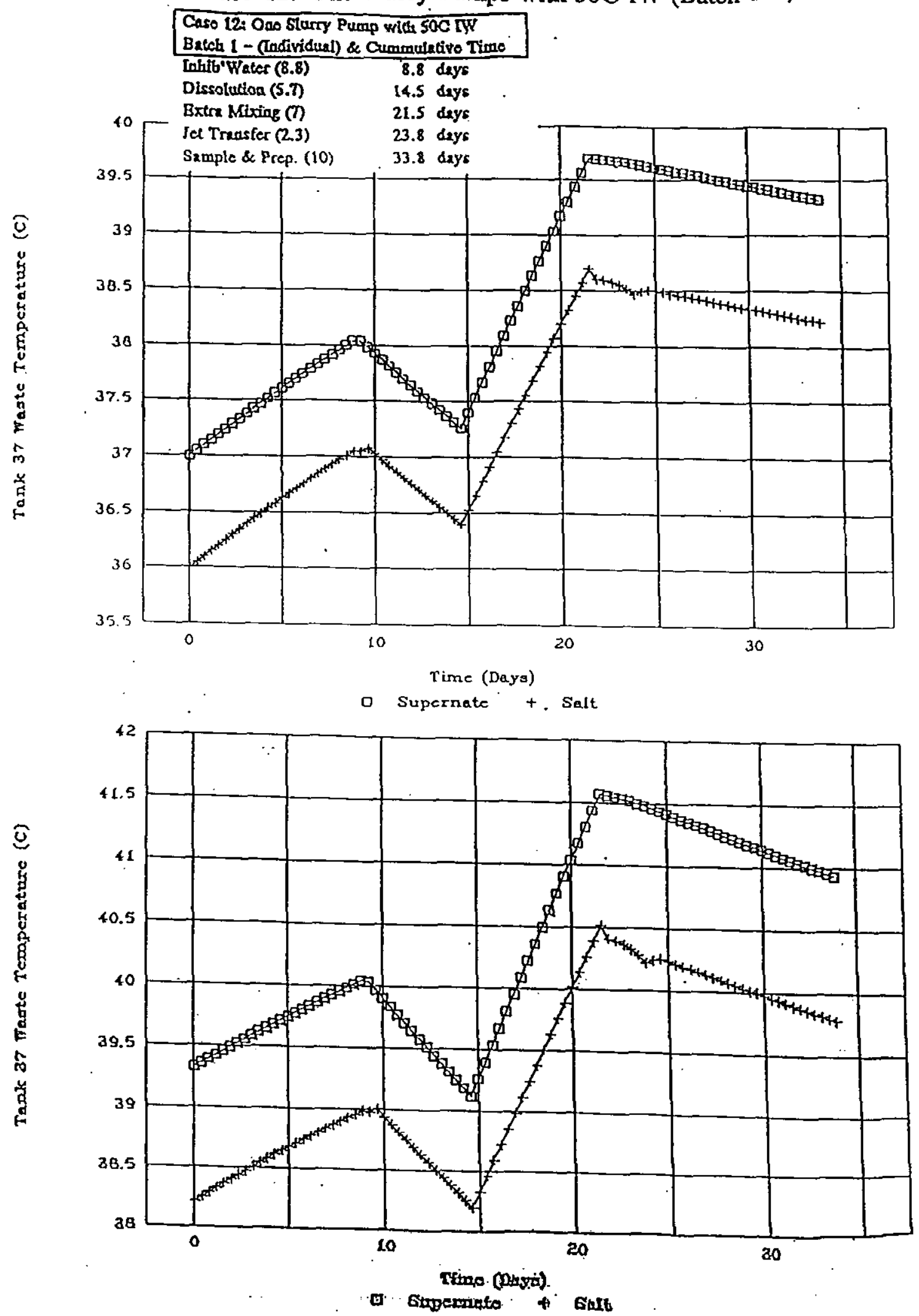
Case 12 One Slurry Pumps with 50C IW (Batch 3-4)
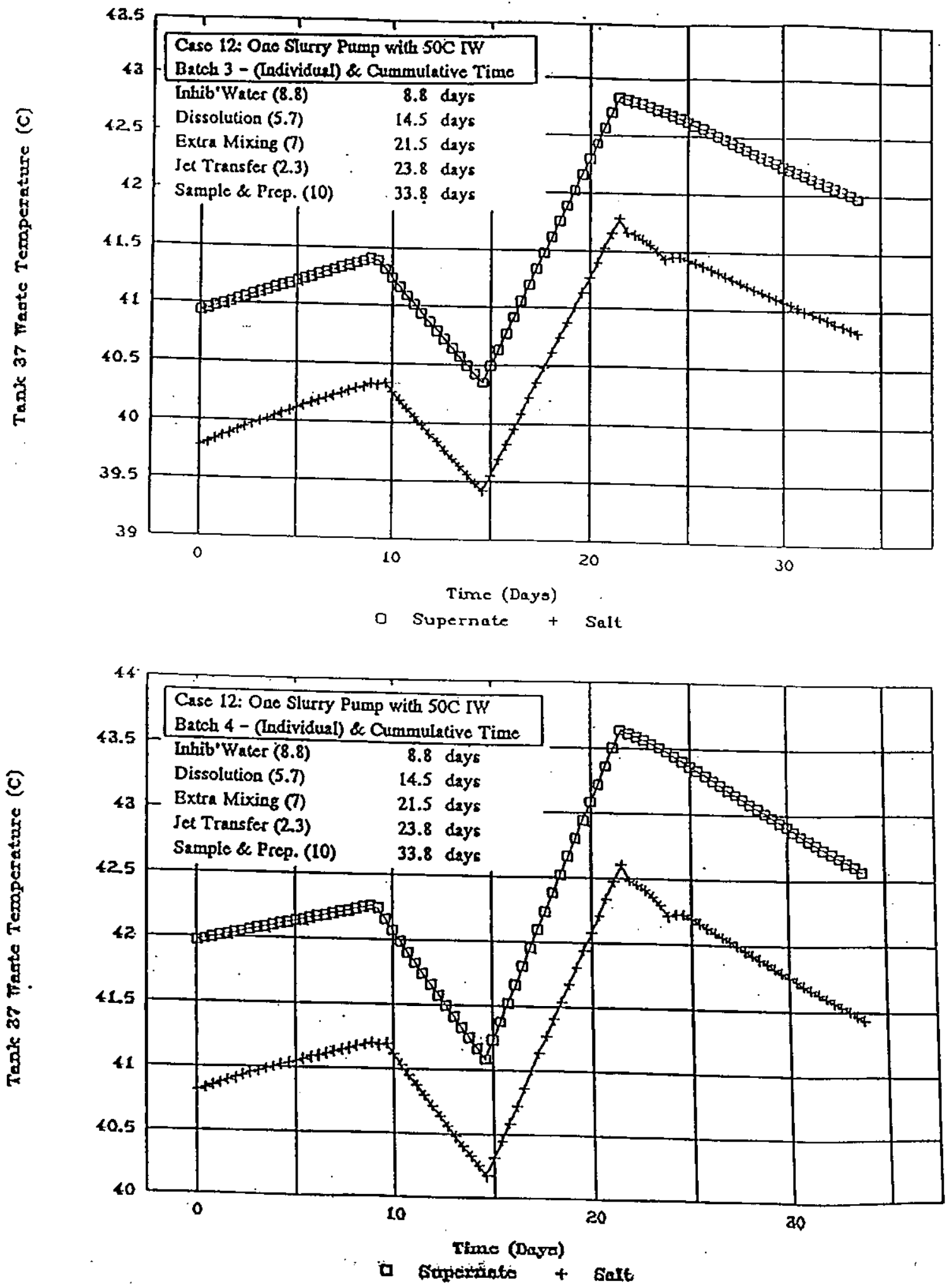
Case 13: Three Flygt Mixers with 50C IW (Batch 1-2)
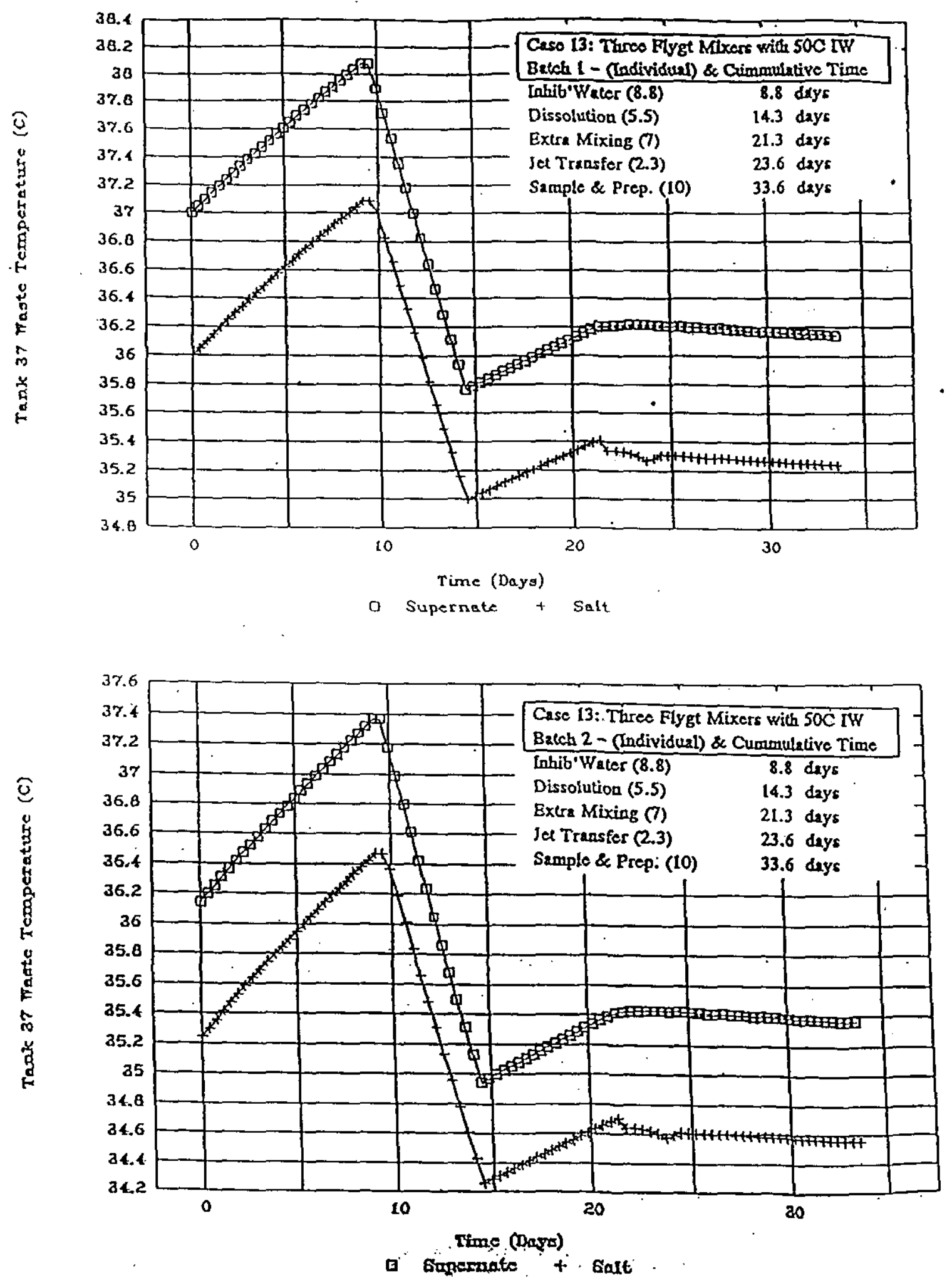
Case 13 Three Flygt Mixers with 50C IW (Batch 3-4)
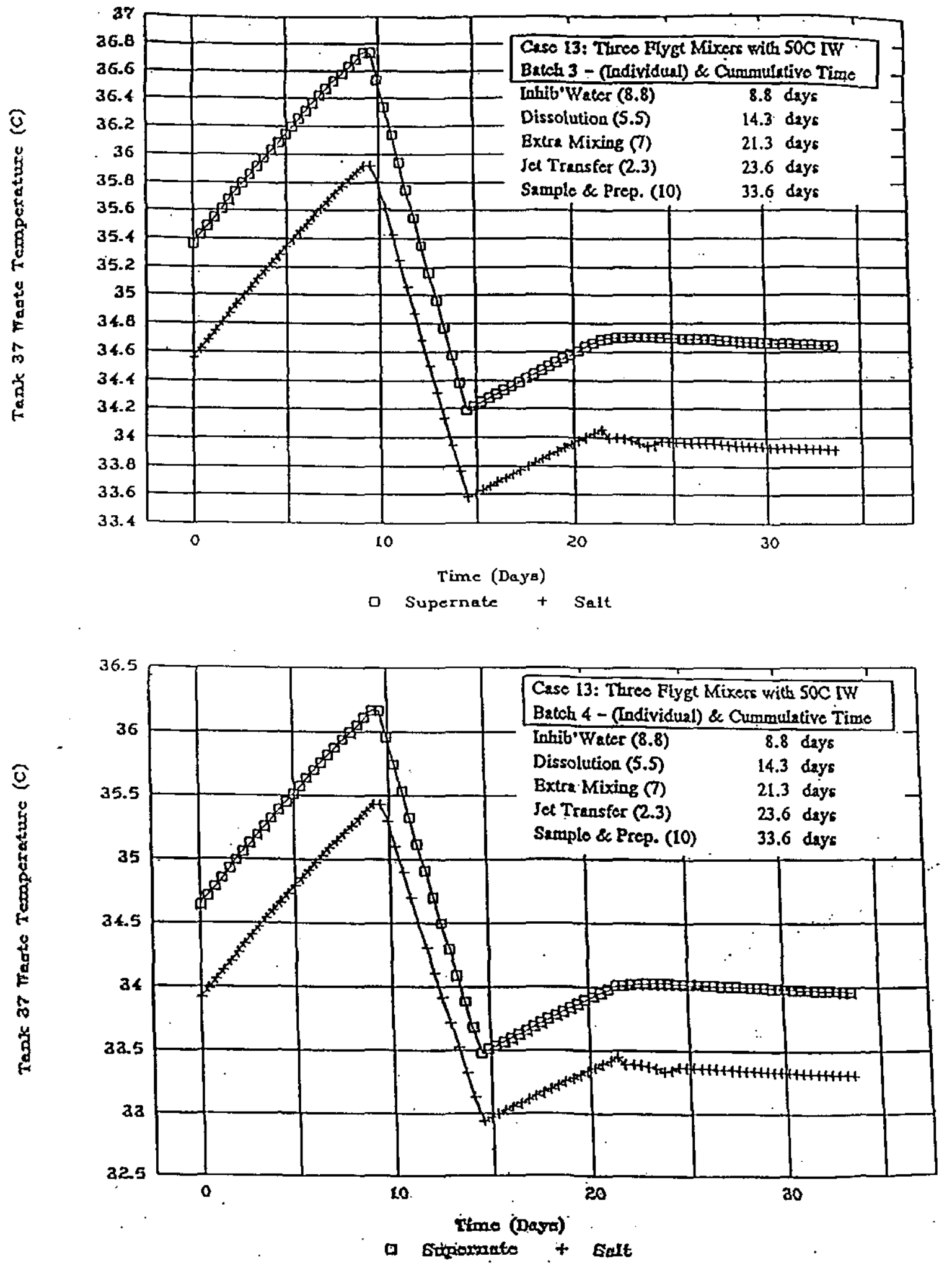
Case 14: Two Flygt Mixers with 75C IW (Batch 1-2)
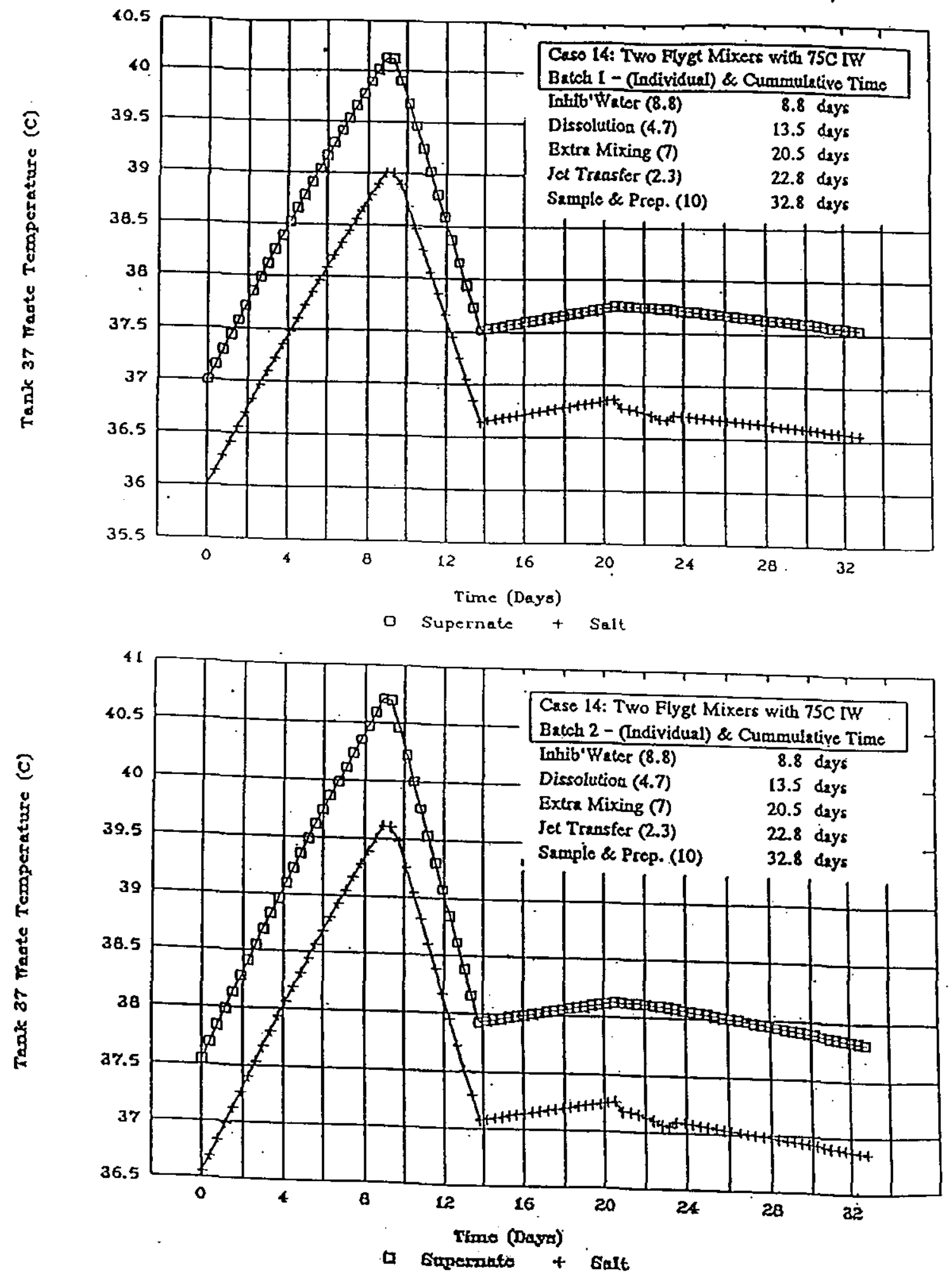


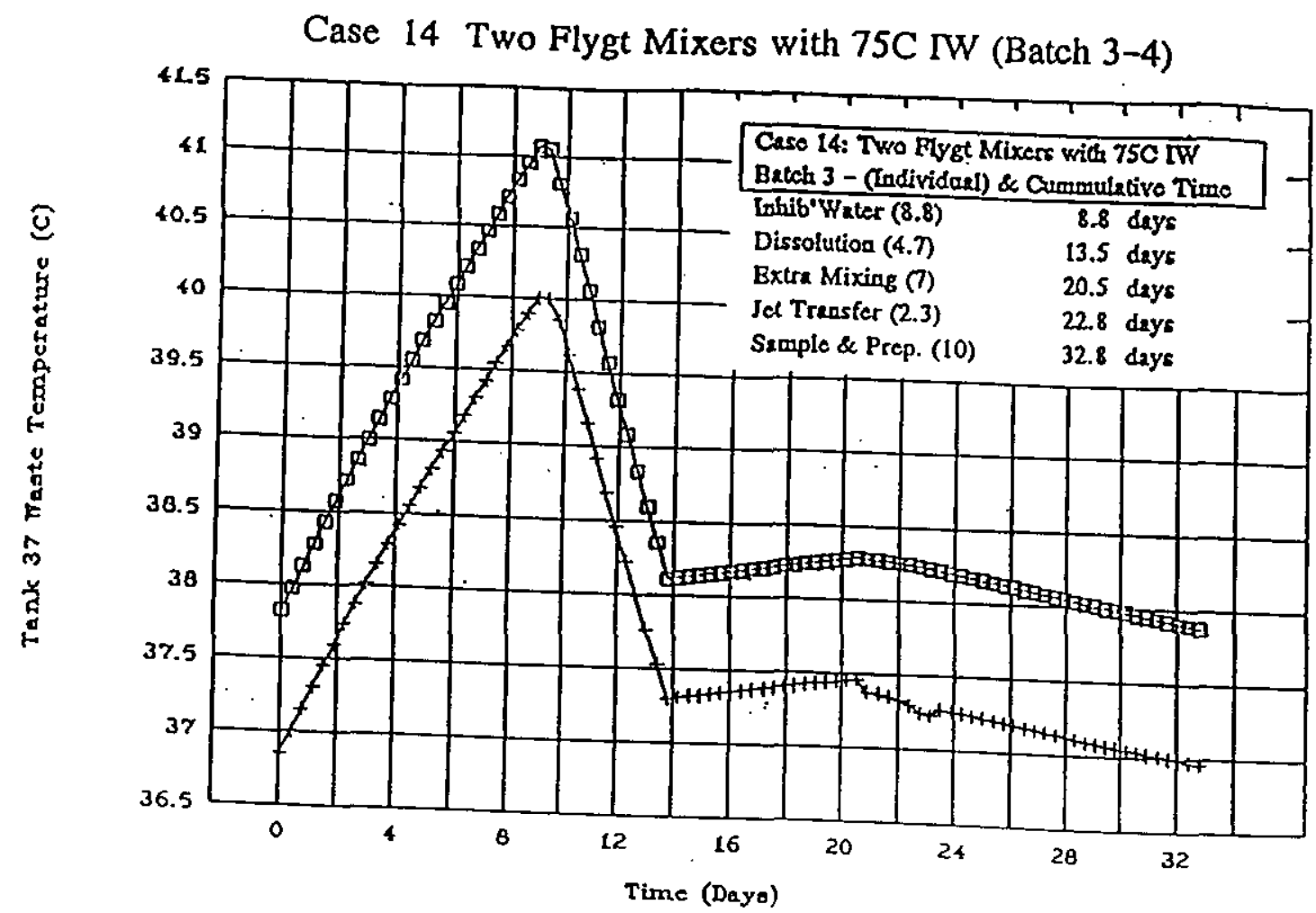

- Supernate + Salt

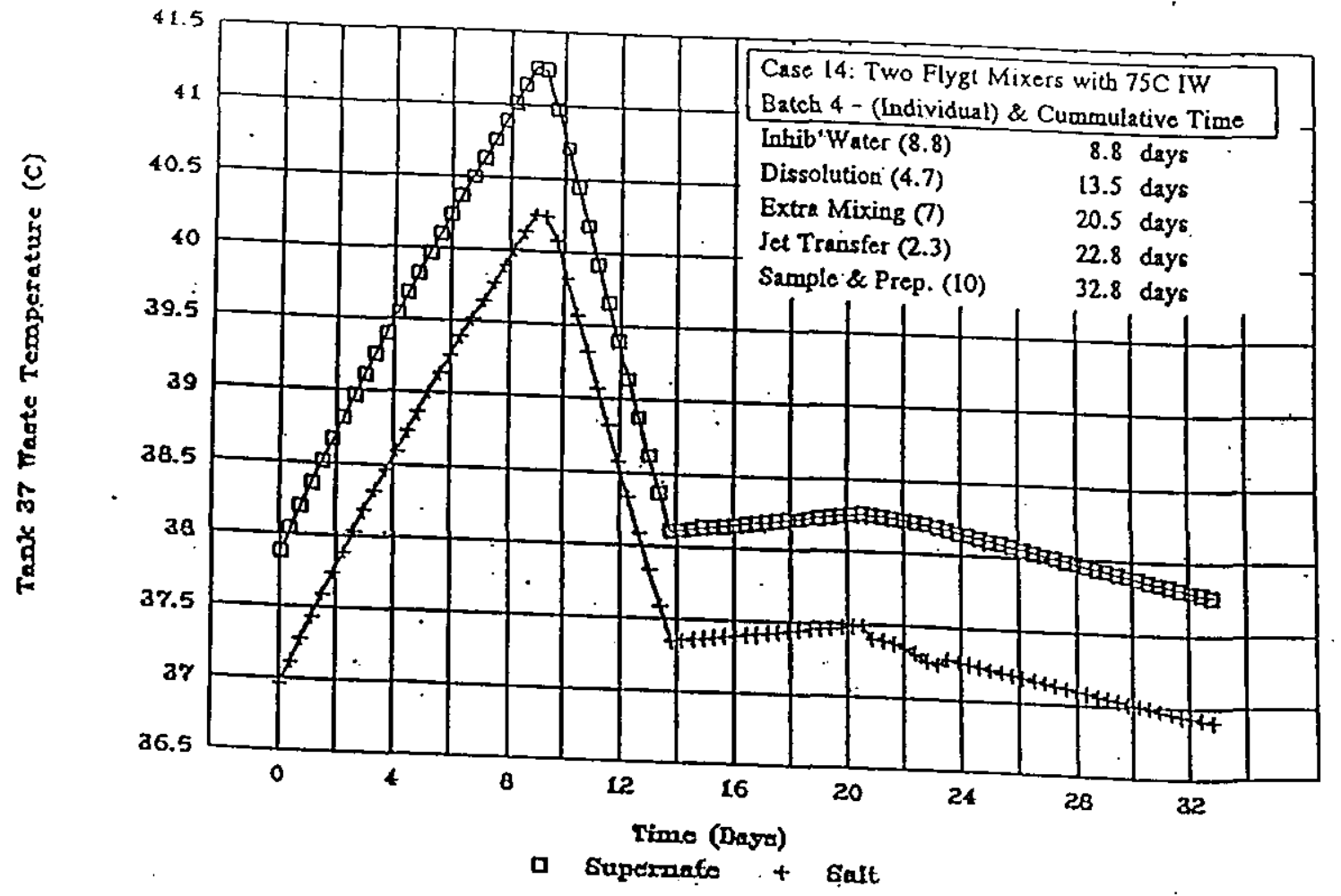


Case 15 One Slurry Pump with 75C IW (Batch 1-2)
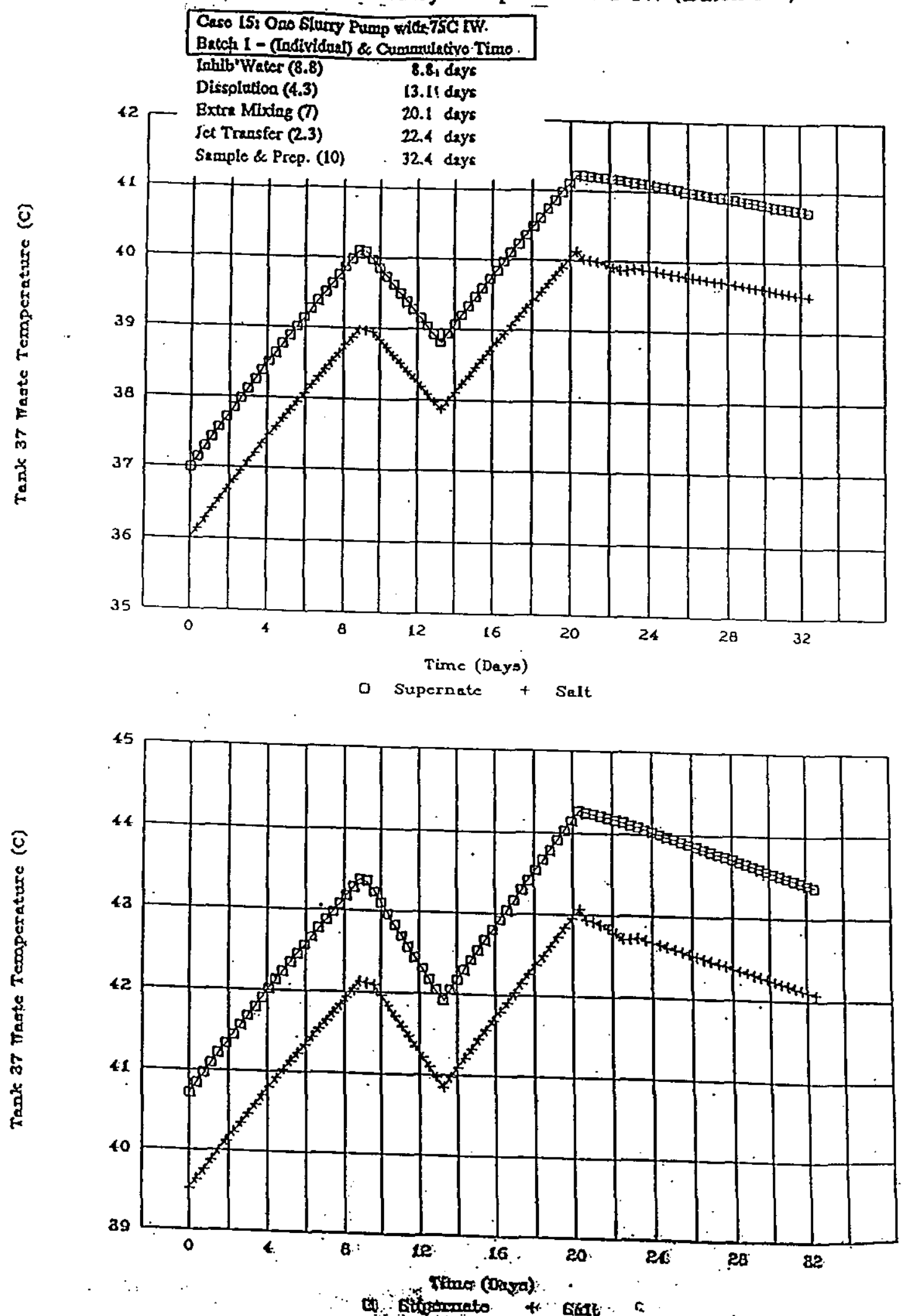
Case 15: One Slurry Pump with 75C IW (Batch 3-4)
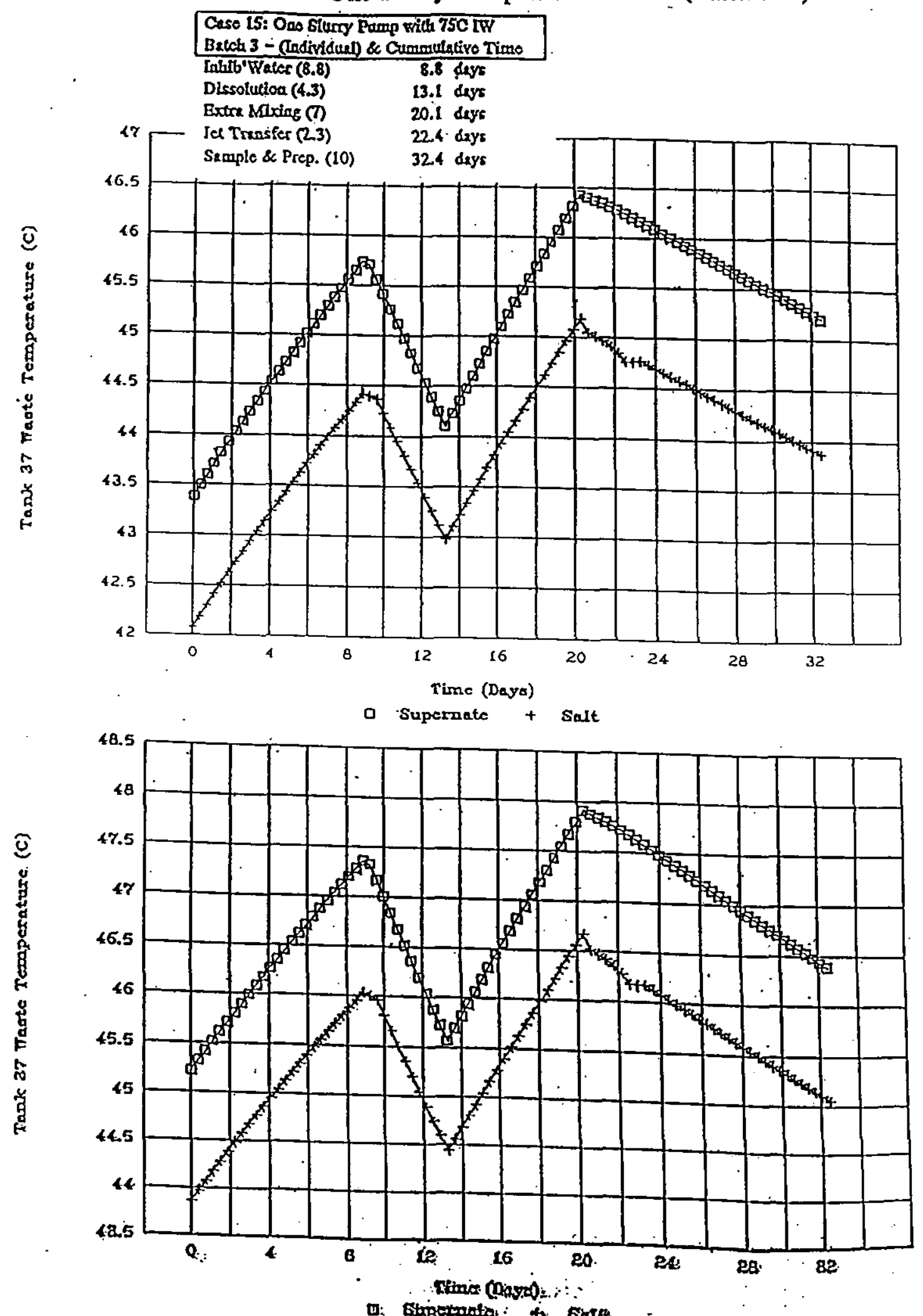
Case 16: Three Flygt Mixers with 75C IW (Batch 1-2)
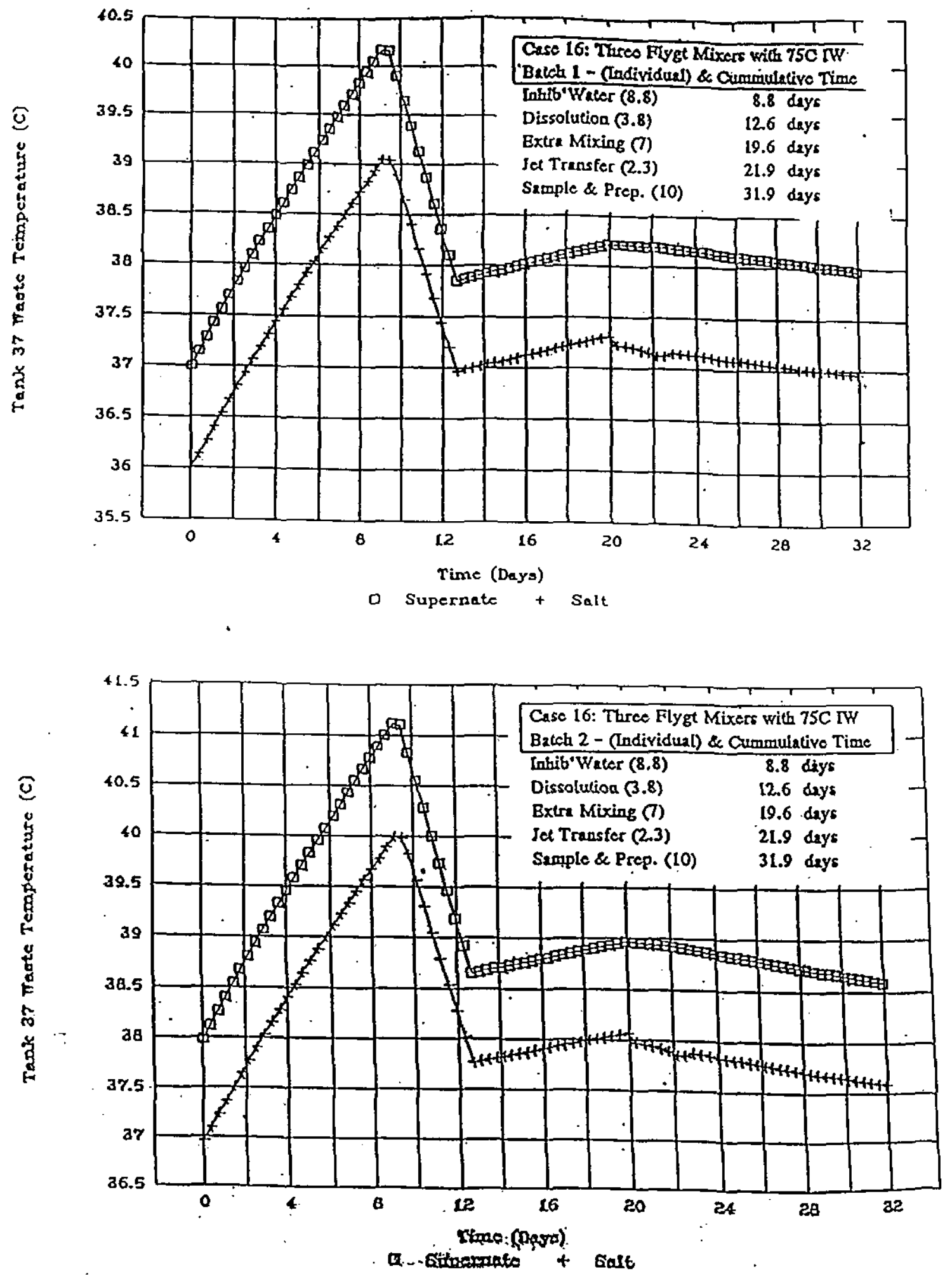
Case 16: Three Flygt Mixers with 75C IW (Batch 3-4).
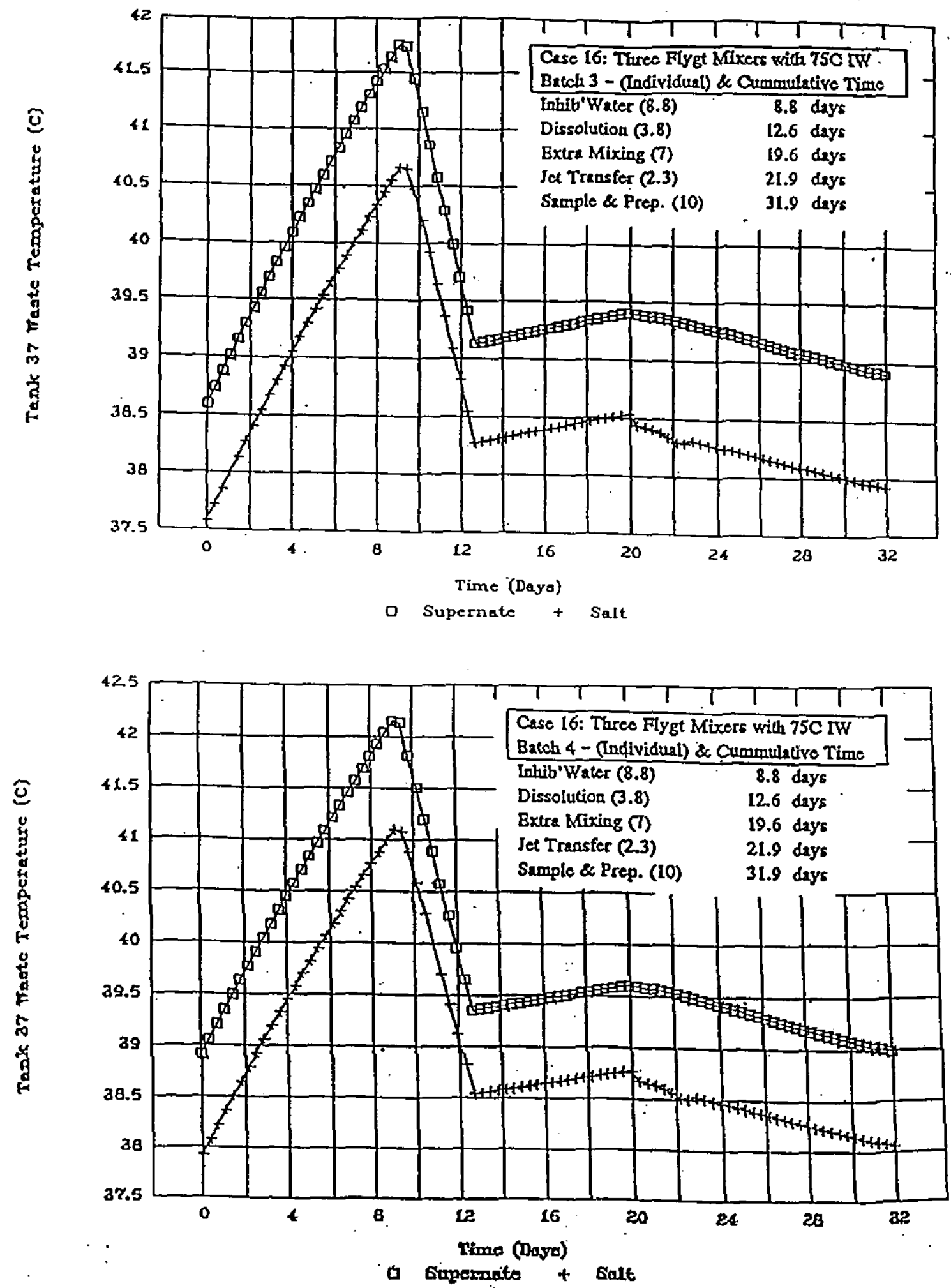